\title{
Magnetic Resonance Pore \\ IMAGING, A NEW TOOL FOR POROUS MEDIA RESEARCH
}

BY

Stefan A. Hertel

A thesis

submitted to Victoria University of Wellington

in fulfilment of the requirements for the degree of

Doctor of Philosophy

Victoria University of Wellington

2015 



\section{Abstract}

Porous media are highly prevalent in nature and span a wide range of systems including biological tissues, chemical catalysts or rocks in oil reservoirs. Imaging of the structure of the constituent pores is therefore highly desirable for life sciences and technological applications. This thesis presents the new development and application of a nuclear magnetic resonance (NMR) technique to acquire high resolution images of closed pores. The technique is a further development of diffusive-diffraction Pulsed Gradient Spin Echo (PGSE) NMR, which has been shown to image the pore auto-correlation function averaged over all pores. Until recently it was conventional wisdom that diffusive-diffraction PGSE NMR can only measure the magnitude of the form factor, due to its similarity to diffraction techniques such as x-ray and neutron scattering. In diffraction applications the loss of phase information is commonly referred to as the "phase problem", which prevents the reconstruction of images of the pore space by inverse Fourier transform. My work is based on a recently suggested modification of the diffusive-diffraction PGSE NMR method, which creates a hybrid between Magnetic Resonance Imaging (MRI) and PGSE NMR. Therefore, we call this approach Magnetic Resonance Pore Imaging (MRPI). We provide experimental confirmation that MRPI does indeed measure the diffractive signal including its phase and thus the "phase problem" is lifted. We suggest a two-dimensional version of MRPI and obtain two-dimensional average pore images of cylindrical and triangular pores with an unprecedented resolution as compared to state of the art MRI. Utilizing a laser machined phantom sample we present images of microscopic pores with triangular shape even in the presence of wall relaxation effects. We therefore show that MRPI is able to reconstruct the pore shape without any prior knowledge or assumption about the porous system under study. Furthermore, we demonstrate 
that the MRPI approach integrates seamlessly with known MRI concepts. For instance we introduce "MRPI mapping" which acquires the MRPI signal for each pixel in an MRI image. This enables one to resolve pore sizes and shapes spatially, thus expanding the application of MRPI to samples with heterogeneous distributions of pores. 


\section{Acknowledgements}

During preparation of my thesis I received help and encouragement from many people and institutions to which I would like to express my gratitude.

First of all, I would like to thank my supervisor Dr. Petrik Galvosas for the guidance, stimulation and support he provided throughout the past three and a half years. His help was especially indispensable regarding the art of mastering the NMR spectrometer and the gradient hardware. Moreover, he extended his support beyond work related issues, which I experienced as exceptionally kind and which made my stay in New Zealand such an enjoyable time.

I would also like to thank Professor Sir Paul Callaghan for providing such a stimulating environment and for entrusting the project for this thesis to me. He sadly passed away, but is remembered vividly in his lab.

There are many people whom I would like to thank from the NMR lab at Victoria University of Wellington. Dr. Mark Hunter helped me to get going on the MRPI project and provided key simulations in the early stages of my work. My colleagues Dr. Bradley Douglas, Dr. Sergej Obruchkov, Phillip Luey and fellow PhD candidates Wilfred Kittler, Stefan Kuczera, Tim Brox, Huabing Liu and Fangrong Zong were an exceptionally talented team. Thanks for all the discussions and the input I received for my work and your immediate help whenever I encountered problems. Thanks also to the workshop Alan, Manu, Nick and Grant who were always stretching their workloads to accommodate new ideas of mine.

Many thanks to Andy Wang and Cather Simpson from the Photon Factory of the University of Auckland for the fruitful collaboration.

I am also very thankful to the examining committee that evaluated my thesis: Prof. 
Bernhard Blümich, Dr. Stefan Hill and Dr. Robin Dykstra, who read my thesis and contributed valuable comments.

Furthermore, I offer my grateful thanks to Victoria University of Wellington for the Victoria PhD scholarship I received.

Very special thanks go to my family who supported me unconditionally.

I dedicate this thesis to Camille and Emilia my new little family. 


\section{Contents}

$\begin{array}{ll}\text { Abstract } & \text { i }\end{array}$

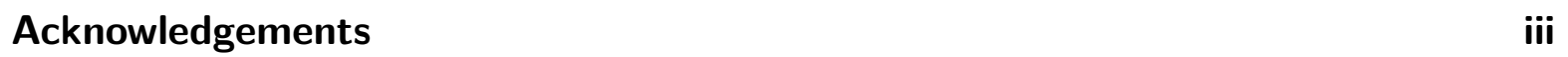

Table of Contents $\quad$ v

List of Figures $\quad$ ix

List of Tables $\quad$ xiii

List of Symbols $\quad$ xv

1 Introduction $\quad 1$

1.1 Porous materials . . . . . . . . . . . . . . . . . . . . . 4

1.2 Molecular self-diffusion . . . . . . . . . . . . . . . 5

1.3 Imaging versus diffraction techniques . . . . . . . . . . . 8

2 Theory and description of NMR diffusion techniques 13

2.1 NMR theory . . . . . . . . . . . . . . . . . . 13

2.1.1 Nuclear magnetization and rotating frame of reference . . . . . . 13

2.1.2 Relaxation ...................... 17

2.1.3 Spin-echo pulse sequence . . . . . . . . . . . . . 20

2.1.4 CPMG pulse sequence . . . . . . . . . . . . . 21

2.1.5 Magnetic field gradients . . . . . . . . . . . . . 23

2.1.5.1 Effective magnetic field gradients . . . . . . . . . 24

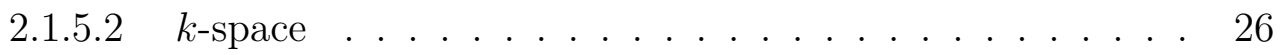


2.1.5.3 q-space . . . . . . . . . . . . . . . . . 27

2.2 NMR diffusive-diffraction techniques . . . . . . . . . . . . . . . . . . . . . 29

2.2.1 Pulsed Gradient Spin Echo (PGSE) NMR . . . . . . . . . . . . 29

2.2.2 Diffusive-diffraction PGSE NMR . . . . . . . . . . . . . . . 33

2.2.3 Magnetic Resonance Pore Imaging (MRPI) . . . . . . . . . . . . . 38

2.2.3.1 MRPI resolution ................ . . 41

2.2.3.2 Blurring .................... . . 43

2.2.3.3 Edge enhancement . . . . . . . . . . . . . . 44

2.3 Inhomogeneous magnetic fields in porous media . . . . . . . . . . 46

2.3.1 Coherence pathways of spin-1/2 nuclei . . . . . . . . . . 46

2.3.2 Influence of relaxation and self-diffusion on coherence pathways . 51

2.3.3 Phase cycles for the selection of desired coherence pathways . . . 52

2.3.3.1 Phase cycle of the spin-echo sequence . . . . . . . . . . 54

2.3.3.2 Phase cycle of the CPMG sequence . . . . . . . . . . 56

2.3.4 Phase Incremented Echo Train Acquisition (PIETA) . . . . . . . . 58

2.4 Multiple Correlation Function technique . . . . . . . . . . . . . . 61

2.4.0.1 Neumann boundary condition . . . . . . . . . . . 63

2.4.0.2 Fourier boundary condition . . . . . . . . . . 66

$\begin{array}{llr}3 & \text { Experimental } & 69\end{array}$

3.1 MRPI pulse sequence design . . . . . . . . . . . . . . . . 69

3.1 .1 1d-MRPI ........................... 70

3.1.1.1 Elimination of unwanted coherence pathways . . . . 73

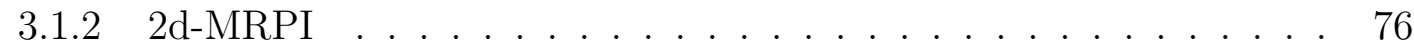

3.1.2.1 2d-Fourier transform approach . . . . . . . 76

3.1.2.2 Radon transform approach . . . . . . . . . . . . . 77

3.1 .3 MRPI mapping . . . . . . . . . . . . . . 79

3.2 Challenges imposed by NMR hardware limitations . . . . . . . . . 80

3.2.1 NMR hardware used . . . . . . . . . . . . . . 80

3.2.2 Resolution limit of the gradient amplifier . . . . . . . . . . . . 81

3.2.3 Further experimental precautions . . . . . . . . . . . . 82 


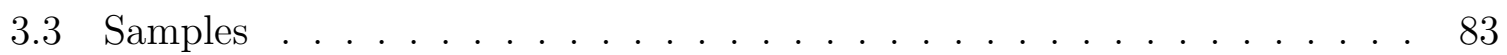

3.3.1 Calibration samples . . . . . . . . . . . . . . . . 83

3.3.2 Cylindrical glass capillaries as phantom samples . . . . . . . . . 84

3.3.3 Hemi-equilateral triangular capillaries as phantom samples . . . . 87

3.3.3.1 Principle of excimer laser dragging . . . . . . . . . 87

3.3.3.2 Production of triangular capillary arrays . . . . . . . . 89

4 Results and discussion $\quad 93$

4.1 Free diffusion . . . . . . . . . . . . . . . . . . . . . . . . . . . 93

4.1 .1 Calibration ......................... 93

4.1 .2 1d-MRPI calibration . . . . . . . . . . . . . . 95

4.1 .3 2d-MRPI calibration . . . . . . . . . . . . . 96

4.2 Cylindrical capillaries . . . . . . . . . . . . . . . . . . . . . 98

4.2.1 Diffusive-diffraction PGSE NMR results . . . . . . . . . . . . . . 98

4.2 .2 1d-MRPI results . . . . . . . . . . . . . . . . 101

4.2.2.1 MRPI convergence to the form factor . . . . . . . . . 104

4.2.2.2 MRPI - PIETA phase cycle . . . . . . . . . . 106

$4.2 .3 \quad 2 \mathrm{~d}-\mathrm{MRPI}$ results $\ldots \ldots \ldots$. . . . . . . . . . . . . . . . 108

4.3 Triangular capillaries . . . . . . . . . . . . . . . . . . . . . . 110

4.3.1 Multiple Correlation Function simulations . . . . . . . . . . . . . 111

4.3 .2 1d-MRPI results . . . . . . . . . . . . . . . . . 114

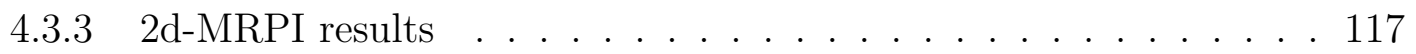

4.4 MRPI mapping . . . . . . . . . . . . . . . . . . . . . . 118

4.4 .1 MRPI 1d-mapping . . . . . . . . . . . . . . 118

4.4 .2 MRPI 2d-mapping . . . . . . . . . . . . . . . 120

5 Conclusions 123

5.1 Future work . . . . . . . . . . . . . . . . . . 125

$\begin{array}{ll}\text { Bibliography } & 127\end{array}$ 



\section{List of Figures}

1.1 Electron microscope image of active carbon as an example of a porous material. . . . . . . . . . . . . . . . . . . . 4

1.2 Fourier relationship of the form factor and the pore density function on the example of a rectangular pore. . . . . . . . . . . . . . . . 10

1.3 Fourier relationship of the average square of the form factor and the pore density auto-correlation function on the example of a rectangular pore. . 11

2.1 Application of an oscillating magnetic field through a solenoid perpendicular to the $B_{0}$ field. . . . . . . . . . . . . . . . . 16

2.2 Magnetization under the influence of a rf-pulse in the rotating frame of reference. . . . . . . . . . . . . . . . . . . 17

2.3 Schematic representation of the free induction decay. . . . . . . . . . . 19

2.4 Spin-echo rf-pulse sequence. . . . . . . . . . . . . . . . . . . . 20

2.5 Scheme of the dynamics of the magnetization in the spin-echo experiment in the rotating frame of reference. . . . . . . . . . . . . . . . . 21

2.6 The CPMG rf-pulse sequence. . . . . . . . . . . . . . . . . . . . 22

2.7 Scheme of a Maxwell coil generating a magnetic field gradient. . . . . . . 24

2.8 Effective magnetic field gradients. . . . . . . . . . . . . . 25

2.9 The pulsed gradient spin echo (PGSE) NMR sequence. . . . . . . . . . . 30

2.10 Sketch of the pore spin density of an isolated closed pore. . . . . . . . 33

2.11 Simulated diffusive-diffraction PGSE NMR signal utilizing cylindrical pores. 36

2.12 Sketch of a matrix of identical cylindrical pores. Shown is the transverse cross-section of the pore system. . . . . . . . . . . . . . . 37 
2.13 Effective gradient schemes of diffusive-diffraction PGSE NMR. . . . . . . 39

2.14 Scheme of the convergence of the trajectory center of mass to the pore center of mass. . . . . . . . . . . . . . . . . . . . . . . . 40 40

2.15 Scheme of a gradient pulse with gradient strength increments. . . . . . . 41

2.16 Effect of blurring on the MRPI pore image. . . . . . . . . . . . . 44

2.17 Effect of edge enhancement on the MRPI pore image. . . . . . . . . . . . 45

2.18 Scheme of the precession of the magnetization vector about the effective magnetic field. . . . . . . . . . . . . . . . . . . . . 47

2.19 CPMG rf-pulse sequence including spin-1/2 coherence pathways. . . . . . 57

2.20 PIETA phase scheme as applied to the CPMG pulse sequence. . . . . . . 58

2.21 PIETA results for ideal and misset rf-pulse durations. . . . . . . . . . . . 61

3.1 Internal gradient compensation utilizing a CPMG like rf-pulse sequence. . 71

3.2 Modified 1d-MRPI pulse sequence with a CPMG like rf-pulse scheme. . . 72

3.3 Time domain signal of the destructive stimulated echo pathway. . . . . . 75

3.4 2d-MRPI pulse scheme. . . . . . . . . . . . . . . . . 77

3.5 Scheme of the Radon transform on the example of a square object. . . . . 78

3.6 MRPI 1d-mapping pulse sequence . . . . . . . . . . . . . . . . . . . 80

3.7 MRPI 2d-mapping pulse sequence . . . . . . . . . . . . . . . . 80

3.8 Scheme showing an artifact introduced by the resolution limit of the gradient amplifier. . . . . . . . . . . . . . . . . . . 81

3.9 Scheme of the gradient pulse distortions and their suppression using ramped gradient pulses. . . . . . . . . . . . . . . . . 83

3.10 Sketch of the cylindrical micro-capillaries used to assemble the NMR phantom.

3.11 Sketch of the capillary sample CYL2 with two compartments of different capillary radii . . . . . . . . . . . . . . . . . 85

3.12 MRI image of the filled capillaries. . . . . . . . . . . . . . . . 86

3.13 Scheme of microgroove pattern machining by excimer laser dragging. . . 88

3.14 Scheme of the height of the mask and the depth of the groove. . . . . . . 88 
3.15 Sketch of hemi-equilateral triangular pores and their alignment into an ordered array. . . . . . . . . . . . . . . . . . . . . . 89

3.16 Optical microscope image of the channel cross-section for two exemplary triangular capillaries. . . . . . . . . . . . . . . . . . . 90

3.17 Sketch of the triangular capillary stack. . . . . . . . . . . . . . . 91

3.18 MRI image of the filled triangular capillaries. . . . . . . . . . . . . . . . 91

4.1 Comparison of spin-echo attenuation for free diffusion using MRPI and diffusive-diffraction PGSE NMR. . . . . . . . . . . . . . . . . . 94

4.2 Signal amplitude obtained by applying the modified 1d-MRPI pulse sequence to measure free self-diffusion. . . . . . . . . . . . . . . . 95

4.3 2d-MRPI applied to PDMS. . . . . . . . . . . . . . . . . . . 97

4.4 Spin-echo attenuation of a one-dimensional extraction from the $2 \mathrm{~d}-\mathrm{MRPI}$ data. . . . . . . . . . . . . . . . . . 97

4.5 Spin-echo signal in the time domain. . . . . . . . . . . . . . . . . . . 98

4.6 Diffusive-diffraction PGSE NMR data obtained by measuring restricted diffusion in the cylindrical capillaries. . . . . . . . . . . . . . . . . . . . . 99

4.7 Comparison experimental and theoretical pore density correlation function.100

4.8 MRPI signal arising from the cylindrical capillaries. . . . . . . . . . . . . 102

4.9 1d projection of the pore density as obtained by MRPI. . . . . . . . . . . 104

4.10 MRPI convergence to the form factor. . . . . . . . . . . . 105

4.11 Coherence transfer pathway map of MRPI PIETA applied to sample CYL1.106

4.12 Comparison of 1d-MRPI without PIETA phase cycling and when PIETA phase cycling is applied. . . . . . . . . . . . . . . 107

4.13 2d-MRPI data and average pore image of the sample CYL1. . . . . . . 109

4.14 One-dimensional cross section through the 2d-MRPI image. . . . . . . . 110

4.15 MCF simulated pore images of the triangular domain. Parameter changed $N_{\mathrm{N}} \ldots \ldots \ldots \ldots \ldots \ldots \ldots \ldots \ldots$

4.16 MCF simulated pore images of the triangular domain. Parameter changed $N_{\mathrm{L}} \ldots \ldots \ldots \ldots \ldots \ldots \ldots \ldots$ 
4.17 MCF simulated pore images of the triangular domain. Parameter changed $N_{\theta} \ldots \ldots \ldots \ldots \ldots \ldots \ldots \ldots$

4.18 1d-MRPI results on the triangular capillaries. . . . . . . . . . . . . . 115

4.19 One-dimensional profile of the hemi-equilateral triangular domain . . . . 116

4.20 Average pore image obtained from the hemi-equilateral triangular capillaries. . . . . . . . . . . . . . . . . . . 118

4.21 Plot of the results of the one-dimensional MRPI mapping experiment. . . 119

4.22 Experimental results of the MRPI 2d-mapping experiment. . . . . . . . . 121 


\section{List of Tables}

2.1 Coherence pathway vectors $\boldsymbol{p}$ of the spin-echo pulse sequence . . . . . . 54

2.2 Phase cycle of the spin-echo pulse sequence. . . . . . . . . . . . 56

2.3 Phase cycle of the CPMG pulse sequence. . . . . . . . . . . . . . . 57

3.1 Phase cycle for the CPMG based MRPI pulse sequence. . . . . . . . . . 76

4.1 Zeros of the NMR signal $E(q)$ obtained from the MRPI and diffusivediffraction PGSE-NMR data. . . . . . . . . . . . . . . . . . 103 



\section{List of Symbols}

\begin{tabular}{ll} 
Symbol & Description \\
$\alpha_{\mathrm{i}}$ & zeros of the cylindrical Bessel function \\
$\beta$ & maximum gradient intensity \\
$\delta$ & gradient pulse width of rectangular \\
& shaped gradient pulse \\
$\delta^{\prime}$ & length of the gradient pulses in the \\
& modified MRPI pulse sequence \\
$\delta_{\mathrm{L}}$ & length of the long gradient pulse \\
$\delta_{\mathrm{N}}$ & length of the narrow gradient pulse \\
$\delta_{\mathrm{R}}$ & length of the read gradient \\
$\delta_{\mathrm{s}}$ & separation between gradient pulses \\
$\Delta$ & PGSE NMR observation time \\
$\gamma$ & gyro-magnetic ratio \\
$\lambda_{\mathrm{n}}$ & eigenvalues \\
$\Lambda$ & rf-pulse rotation matrix \\
$\mu$ & magnetic moment \\
$\omega_{0}$ & Larmor frequency \\
$\omega_{\mathrm{rf}}$ & carrier angular frequency \\
$\Omega$ & nutation angular frequency \\
$\rho_{\mathrm{p}}(r)$ & confining domain \\
& pore spin density function \\
\hline &
\end{tabular}




\begin{tabular}{|c|c|}
\hline$\rho_{0}^{*}(r)$ & $\begin{array}{l}\text { pore spin density auto-correlation func- } \\
\text { tion }\end{array}$ \\
\hline$\sigma_{\mathrm{x}}$ & standard deviation \\
\hline$\tau$ & rf-pulse separation \\
\hline$\theta$ & angle \\
\hline$\chi$ & magnetic susceptibility \\
\hline$\xi_{-1}$ & geometry factor \\
\hline$b$ & generalized gradient parameter \\
\hline$B$ & magnetic field \\
\hline$B_{0}$ & polarizing magnetic field \\
\hline$B_{1}$ & magnetic field of rf-pulses \\
\hline$B_{0}^{\text {loc }}$ & local polarizing magnetic field \\
\hline$D$ & self-diffusion coefficient \\
\hline$D_{\text {app }}$ & apparent self-diffusion coefficient \\
\hline$E(q)$ & normalized spin-echo amplitude \\
\hline$f(t)$ & time dependence of gradient scheme \\
\hline $\boldsymbol{F}$ & free precession matrix \\
\hline$F O V$ & field of view \\
\hline$g$ & $\begin{array}{l}\text { gradient strength of background gradi- } \\
\text { ents }\end{array}$ \\
\hline$G$ & $\begin{array}{l}\text { gradient strength of pulsed field gradi- } \\
\text { ents }\end{array}$ \\
\hline$G_{\mathrm{L}}$ & $\begin{array}{l}\text { gradient strength of the long gradient } \\
\text { pulse }\end{array}$ \\
\hline$G_{\mathrm{N}}$ & $\begin{array}{l}\text { gradient strength of the narrow gradi- } \\
\text { ent pulse }\end{array}$ \\
\hline$G *$ & $\begin{array}{l}\text { effective magnetic field gradient } \\
\text { strength }\end{array}$ \\
\hline$\tilde{G}$ & spatial dependence of magnetic field \\
\hline$h$ & generalized relaxation parameter \\
\hline
\end{tabular}


$\hbar \quad$ Plancks constant divided by $2 \pi$

$\mathcal{H}_{\text {lab }} \quad$ Hamiltonian in the laboratory reference

frame

$\mathcal{H}_{\text {rot }} \quad$ Hamiltonian in the rotating reference

frame

$i(t) \quad$ current in the gradient coil

I net nuclear spin

$I_{f} \quad$ second moment of the temporal gradi-

ent profile

$k \quad$ reciprocal space vector

$k_{\mathrm{B}} \quad$ Boltzmann constant

$L \quad$ dimension of a pore

$L_{\text {coil }} \quad$ inductance of the gradient coil

$m \quad$ eigenvalues of the spin operator

$\boldsymbol{m}$ magnetization density vector

$M \quad$ magnetization

$M_{+,-, 0} \quad$ magnetization of the coherences $+1,-1$

and in $z$-direction

$M_{0} \quad$ equilibrium magnetization

$M_{x, y} \quad$ magnetization in the $x, y$-plane

$M_{x^{\prime}, y^{\prime}} \quad$ magnetization in the $x, y$-plane in the

rotating frame of reference

$M_{z} \quad$ magnetization $z$ direction

$M_{\mathrm{w}} \quad$ molecular weight

$n \quad$ number of spin-echos

$N_{+} \quad$ spin excess in the lower energy state

$N_{-} \quad$ spin excess in the higher energy state

$N_{\theta} \quad$ number of radial profiles

$N_{\mathrm{G}} \quad$ number of pulsed gradient steps

$N_{\mathrm{L}} \quad$ number of long gradient segments 
$N_{\text {scan }} \quad$ number of scans

$p \quad$ generalized diffusion parameter

$\boldsymbol{p} \quad$ coherence pathway vector

$p_{\mathrm{k}} \quad$ coherence order

$P\left(\boldsymbol{r}, \boldsymbol{r}_{0}, t\right)$ propagator

$\bar{P}(\boldsymbol{R}, t) \quad$ average propagator

$P(r, \theta) \quad$ profile of the spin density

$\Delta P \quad$ cumulative coherence change

$\boldsymbol{q} \quad$ scattering wave vector

$q_{\mathrm{G}} \quad$ generalized gradient intensity

$\boldsymbol{r} \quad$ position vector

$\boldsymbol{r}_{\mathrm{p}} \quad$ local pore coordinate

$\boldsymbol{R}$ displacement vector

$S \quad$ NMR signal

$S_{0}(q) \quad$ form factor

$t \quad$ time

$t_{\mathrm{p}} \quad$ rf-pulse length

$t_{\text {ramp }} \quad$ ramp time of gradient pulses

$T \quad$ length of the MRPI gradient scheme

$T_{\mathrm{K}} \quad$ temperature

$T_{1} \quad$ longitudinal relaxation time

$T_{2} \quad$ transverse relaxation time

$T_{2}^{*} \quad$ transverse relaxation time due to inho-

mogeneities

$T_{2}^{\text {eff }} \quad$ effective transverse relaxation time

$t_{\mathrm{E}} \quad$ spin-echo time

$u_{\mathrm{n}}(\boldsymbol{r}) \quad$ eigenfunctions of the confining domain

$U(t) \quad$ induced voltage

$U_{\max } \quad$ maximum voltage of the gradient amplifier 
V volume

$x_{\max } \quad$ maximum of the edge enhancement

$x_{\mathrm{cm}} \quad$ center of mass of Brownian motion 



\section{Introduction}

Porous materials consist of a solid or semisolid matrix containing void spaces which are filled with fluids (Dullien (1992)). Hence, one may consider wood, concrete and biological cells as everyday porous materials. The capacity of the pore space to load or capture fluids together with the ability of the pore walls to interact with these fluids makes porous media important for many applications like catalysis, oil exploration and medical diagnosis.

In porous media research one regularly studies porous structures at the micrometer scale and below. For most non-invasive imaging techniques such as Magnetic Resonance Imaging (MRI) and x-ray tomography this length scale is either inaccessible due to fundamental resolution limitations (Mansfield and Morris (1982)) or due to the deposition of high radiation doses (Als-Nielsen and McMorrow (2011)). Thus, the most direct structure determination is provided by diffraction techniques such as x-ray scattering (Als-Nielsen and McMorrow (2011)) and neutron scattering (Svergun et al. (1987)). It has been shown that Pulsed Gradient Spin Echo (PGSE) NMR can also be understood as such a diffraction technique (Callaghan et al. (1991)).

These techniques are able to obtain properties of the form factor, a function which concisely describes the material in the Fourier space. However, using diffraction techniques only the modulus of the form factor is measured, which prevents one from obtaining images by a simple inversion of acquired data. This problem is commonly known as the "phase problem" and refers to the fact that a diffractogram only contains amplitude information, but no phase information (Als-Nielsen and McMorrow (2011), Callaghan et al. (1991)).

Recent advances of PGSE NMR based methods have been shown to lift this severe 
limitation. A general approach suggested by Laun et al. (2011) predicts that a modification of a single PGSE-NMR experiment is sufficient to acquire form factors for arbitrary geometries. Shemesh et al. (2012) introduced a synergistic approach using two double PGSE (d-PGSE) experiments. It enables one to obtain the full form factor, although it remains limited to certain pore symmetries (Kiselev and Novikov (2013)). This approach was later extended theoretically by Kuder and Laun (2012) to arbitrary pore geometries, although in practice this approach would have limitations related to poor signal-to-noise.

This PhD work presents innovative approaches to resolve the considerable experimental challenges of the method of Laun et al. (2011). These innovations led to the first experimental results on cylindrical micro-capillaries and the suggestion of the name Magnetic Resonance Pore Imaging (MRPI) (Hertel et al. (2013)). Independently, Kuder et al. (2013) showed that the MRPI approach is working for millimeter sized triangular capillaries utilizing hyper-polarized xenon gas and therefore showed the practicability of the method for arbitrary pore geometries. However, the proof of feasibility of MRPI on the micrometer scale for non-point symmetric pore shapes remained an open challenge. This challenge was met as a part of this $\mathrm{PhD}$ work by developing a fabrication method for triangular micro-capillaries. Using this phantom sample, Hertel et al. (2015b) obtained images of hemi-equilateral triangular pores using their MRPI approach. Furthermore, Hertel et al. (2015a) suggested the MRPI mapping experiment and experimentally confirmed it as a promising extension of MRPI towards practical applications. This technique allows one to map the MRPI signal onto MRI images as published by Hertel et al. (2015a) for 1d-MRI images and by Hertel et al. (2015b) for 2d-MRI images. The following chapters show the implementation of these innovations on a commercially available micro-imaging NMR system and applications to selected porous materials.

Chapter 1 introduces the theory of porous materials, the theory of self-diffusion of molecules as well as the principles of imaging and diffraction techniques. A deep understanding of these diverse scientific fields is necessary for the discussion of the MRPI technique.

Chapter 2 presents the necessary theory of NMR and diffusive-diffraction PGSE 
NMR from which MRPI emerges as a modification. Despite being a modification of PGSE NMR, MRPI provides new information which requires additional theoretical insight which will be discussed. Moreover, the effects of blurring and edge enhancement are introduced and and the specific effects on the MRPI images are highlighted in this chapter. In addition, sec. $\mathbf{2 . 3}$ describes the dynamics of the nuclear magnetization in inhomogeneous magnetic fields, which are common in porous media. Since our approach to implement MRPI is based on a train of rf-pulses, the understanding of these dynamics becomes necessary to overcome experimental artifacts. Moreover, this section introduces an advanced rf-pulse phase cycling scheme called Phase Incremented Echo Train Acquisition (PIETA) which may be required if many rf-pulses are applied. In sec. 2.4 a mathematical tool is introduced to simulate NMR diffusion experiments in closed pore systems. This so called Multiple Correlation Function (MCF) technique is a matrix based technique that provides more accurate results than Monte Carlo simulations in a fraction of the computing time.

Chapter 3 contains a detailed description of the applied methods as well as the sample preparation techniques. The original MRPI pulse sequence as introduced by Laun et al. (2011) poses several experimental challenges which may prevent its application to porous materials in practice. Thus, we developed an adapted MRPI pulse sequence, which applies non-standard approaches to both hardware and software implementation as well as for the sample preparation. In sec. 3.1 the modified MRPI pulse sequence design is described including several experimental artifacts and their prevention. Additionally, the concepts of 2d-MRPI and MRPI mapping are introduced which are an extension of the originally proposed method of Laun et al. (2011).

Chapter 4 shows the experimental results of applying the MRPI pulse sequence to model porous samples and the comparison of the experimental data to theory and simulations. Section 4.1 presents procedures and experiments for a thorough calibration of the experimental setup. Following calibration, the MRPI pulse sequence was applied to custom built model porous samples. Section 4.2 focuses on the experimental results of MRPI on cylindrical micro-capillaries while sec. 4.3 focuses on triangular micro-capillaries. In sec. 4.4 the MRPI mapping pulse sequence is applied to a sample 
containing cylindrical capillaries of two different sizes. These results and their potential in the context of porous media research are discussed in chap. 5 .

\subsection{Porous materials}

Porous materials consist of a solid or semisolid matrix containing void spaces which are filled with fluids (Dullien (1992)). The spaces free of solid are called "pores" which contain the pore fluid such as air, water, hydrocarbons etc. or a mixture of fluids (Dullien (1992)). Figure 1.1 shows the pores and the solid matrix on the example of activated carbon, a porous solid used for adsorption and purification applications (Ruthven (1984)). The structure of the pores including their shape, connectivity and

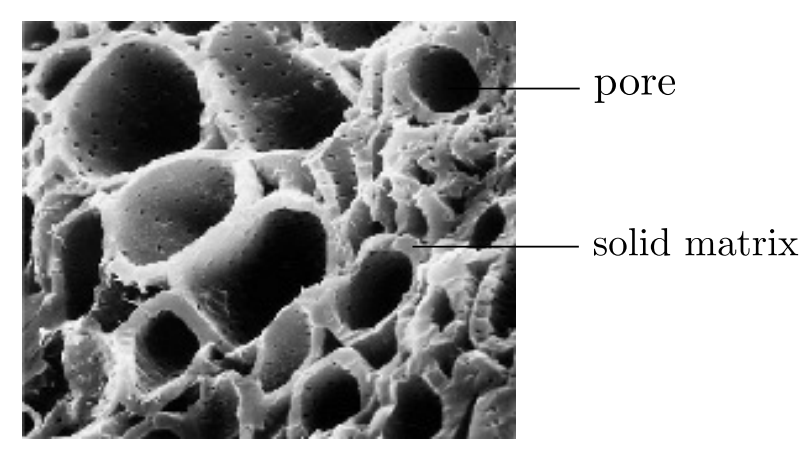

Figure 1.1: Electron microscope image of active carbon as an example of a porous material. The separation between pore space containing the pore fluid and the solid matrix is indicated. Reproduced with permission from Kalies (2009).

their size is crucial for understanding their interaction with the pore fluid (Ruthven (1984)). This interaction determines the performance of porous media in natural systems and technological applications including the ability to transport fluids through the pore space and the capacity of the pores to store fluids. According to the International Union of Pure and Applied Chemistry (IUPAC) (Rouquerol et al. (1994)) porous media are classified according to the width of their pores. In case of cylindrical pores this is the diameter and in case of slit pores it is the distance between the pore walls. The different categories recommended by IUPAC are

- micro-pores: $d<2 \mathrm{~nm}$ 
- meso-pores: $2 \mathrm{~nm} \leq d \leq 50 \mathrm{~nm}$

- macro-pores: $d>50 \mathrm{~nm}$

In recent literature the name nano-pores is used regularly and refers to pores of sizes in between $1 \mathrm{~nm} \leq d \leq 10 \mathrm{~nm}$. The relevant materials considered in this thesis are macro-porous with pore widths $d>1 \mu \mathrm{m}$.

\subsection{Molecular self-diffusion}

In this work, diffusing molecules will be the "agents" exploring the pore space and will enable the imaging of the pore shape by tracking their displacements using a novel NMR method. Diffusion is the random translational motion of particles from one part of the sample to another (Crank (1975)). It was first mathematically described by Fick (1855). Ficks laws assume concentration gradients of physically or chemically discernible particles and the resulting transport of matter by which thermal equilibrium is established.

Self-diffusion on the other hand is the diffusion of particles in the absence of any such concentration gradient. It can still be described by Ficks laws if one considers particles which are "labeled", but are otherwise equal to each other concerning all physical parameters. It describes the random motion of particles due to interactions which arise through the collisions with other particles in the sample. It was first experimentally observed by the botanist Robert Brown in 1827 and is therefore often called Brownian motion.

The function describing the motion of the particles is called the propagator $P\left(\boldsymbol{r}, \boldsymbol{r}_{0}, t\right)$, which is the probability density to find a particle at position $\boldsymbol{r}$ after time $t$, when it initially started at position $\boldsymbol{r}_{0}$ at $t=0$ (Neuman (1974)). Ficks second law also known as the diffusion equation is given by

$$
\frac{\partial P\left(\boldsymbol{r}, \boldsymbol{r}_{0}, t\right)}{\partial t}=D \nabla^{2} P\left(\boldsymbol{r}, \boldsymbol{r}_{0}, t\right)
$$

where $D$ is the self-diffusion coefficient and $\nabla^{2}=\nabla \cdot \nabla$ is the Laplace operator. Strictly, eq. 1.1 only holds under the assumption of an isotropic self-diffusion process, in which 
case $D$ reduces to a scalar. A more general expression can be found in Callaghan (2011). One may solve eq. 1.1 for $P\left(\boldsymbol{r}, \boldsymbol{r}_{0}, t\right)$ when assuming that all particles initially start in one point given by the initial condition $P\left(\boldsymbol{r}, \boldsymbol{r}_{0}, t=0\right)=\delta\left(\boldsymbol{r}-\boldsymbol{r}_{0}\right)$, and by specifying the appropriate boundary conditions for the system under study. In case of an infinitely extended medium the function $P\left(\boldsymbol{r}, \boldsymbol{r}_{0}, t\right)$ is a Gaussian given by

$$
P\left(\boldsymbol{r}, \boldsymbol{r}_{0}, t\right)=\frac{1}{\sqrt{(4 \pi D t)^{3}}} \exp \left\{-\frac{\left(\boldsymbol{r}-\boldsymbol{r}_{0}\right)^{2}}{4 D t}\right\}
$$

which is the fundamental solution of the diffusion equation (eq. 1.1).

Often the solution of a physical problem requires the knowledge of the diffusion behavior expected for a randomly chosen particle. This behavior is contained in the average propagator $\bar{P}(\boldsymbol{R}, t)$, which is obtained by integrating the propagator $P\left(\boldsymbol{r}, \boldsymbol{r}_{0}, t\right)$ over all starting positions $\boldsymbol{r}_{0}$ and including the probability density $p\left(\boldsymbol{r}_{0}\right)$ to start at each of these positions. Introducing the displacement vector $\boldsymbol{R}=\boldsymbol{r}-\boldsymbol{r}_{0}$ one obtains

$$
\bar{P}(\boldsymbol{R}, t)=\int_{V} P\left(\boldsymbol{r}_{0}+\boldsymbol{R}, \boldsymbol{r}_{0}, t\right) p\left(\boldsymbol{r}_{0}\right) \mathrm{d} \boldsymbol{r}_{0}
$$

The average propagator is the probability density to find a randomly chosen particle displaced by the vector $\boldsymbol{R}$ after time $t$. For the case of an isotropic, infinitely extended medium the propagator $P\left(\boldsymbol{r}, \boldsymbol{r}_{0}, t\right)$ is given by eq. 1.1 and the probability density $p\left(\boldsymbol{r}_{0}\right)$ is constant. Thus, the average propagator is equally given by a Gaussian centered at the origin given by

$$
\bar{P}(\boldsymbol{R}, t)=\frac{1}{\sqrt{(4 \pi D t)^{3}}} \exp \left\{-\frac{\boldsymbol{R}(t)^{2}}{4 D t}\right\} .
$$

Hence, the mean displacement is zero as required by the random nature of the process. However, the second moment given by

$$
\left\langle\boldsymbol{R}^{2}(t)\right\rangle=\int_{V} \boldsymbol{R}^{2} \bar{P}(\boldsymbol{R}, t) \mathrm{d} \boldsymbol{r}
$$

is non-zero. By inserting the average propagator for free diffusion as given by eq. 1.4 
into eq. 1.5 one arrives at Einsteins relation for the mean-squared displacement in three dimensions

$$
\left\langle\boldsymbol{R}^{2}(t)\right\rangle=6 D t
$$

In case of restrictions to the motion of the particles such as pore walls the propagator $P\left(\boldsymbol{r}, \boldsymbol{r}_{0}, t\right)$ has to be re-evaluated. Since the particles may be reflected at the boundary, this process is often called reflected Brownian motion (Grebenkov (2006)). The boundary condition is given by

$$
0=D \boldsymbol{n} \nabla P+\left.\rho P\right|_{\mathrm{s}}
$$

where $\boldsymbol{n}$ denotes the unit vector which is normal to the surface $S$ and $\rho$ describes the probability of a particle to be absorbed or transmitted through this surface.

When the absorption probability $\rho$ is zero, the boundary is assumed to be perfectly reflecting. This case is also called the Neumann boundary condition. On the other hand the Robin or Fourier boundary condition, where $\rho$ is finite, describe situations where particles get absorbed or transmitted through the boundary (Grebenkov (2008)). For example, a glass surface may be a perfectly reflecting boundary to water molecules and would therefore require the Neumann boundary condition, while a biological cell membrane may partially transmit water molecules and is described by the Fourier boundary condition. Note that the case of infinite absorption is known as Dirichlet boundary condition, but it will not be explored further here.

One ansatz to solve the diffusion equation eq. 1.1 is to expand the propagator $P\left(\boldsymbol{r}, \boldsymbol{r}_{0}, t\right)$ as a sum of the eigenfunctions $u_{\mathrm{n}}(\boldsymbol{r})$ of the confining domain given by

$$
P\left(\boldsymbol{r}, \boldsymbol{r}_{0}, t\right)=\sum_{\mathrm{n}=0}^{\infty} u_{\mathrm{n}}^{*}\left(\boldsymbol{r}_{0}\right) u_{\mathrm{n}}(\boldsymbol{r}) \exp \left\{-D \lambda_{\mathrm{n}} t\right\}
$$

where $\lambda_{\mathrm{n}}$ are the eigenvalues (Crank (1975)). Through the eigenfunctions of the domain, the functions $P\left(\boldsymbol{r}, \boldsymbol{r}_{0}, t\right)$ and $\bar{P}(\boldsymbol{R}, t)$ contain information about the confining pore structure. Especially, the average propagator $\bar{P}(\boldsymbol{R}, t)$ is important for NMR diffusion studies, 
since it can be shown that the PGSE NMR signal amplitude is directly related to this function (Kärger and Heink (1983)). Moreover, an expansion similar to eq. 1.8 is used in sec. 2.4 for the nuclear magnetization to lay the basis of the simulation technique used to model the MRPI signal amplitude.

\subsection{Imaging versus diffraction techniques}

The MRPI method presented in this thesis can be seen as an imaging method in disguise, a property which will be discussed in sec. 2.2.3 (Laun et al. (2012)). At the same time it is a modification of the diffraction technique called diffusive-diffraction PGSE NMR. Hence, it may be important to understand the difference between imaging techniques and diffraction techniques in general.

Suppose that an object has a specific structural density $\rho_{0}(\boldsymbol{r})$. This quantity can assume values such as photon absorber density in light microscopy and X-ray tomography or the density of proton spins in MRI. In imaging one aims to reconstruct $\rho_{0}(\boldsymbol{r})$ as close as possible to the original distribution. However, the size of the pores in many porous media is beyond the resolution limit of conventional imaging techniques and one may have to resort to diffraction techniques.

Consider the case of a pore filled with fluid. In case the fluid is homogeneous the function $\rho_{0}(\boldsymbol{r})$ is equal to the pore shape. If one performs an inverse Fourier transform of this pore shape one obtains the form factor of the pore given by (Callaghan (2011))

$$
S_{0}(\boldsymbol{k})=\int_{V} \rho_{0}(\boldsymbol{r}) \exp \{\mathrm{i} 2 \pi \boldsymbol{k} \cdot \boldsymbol{r}\} \mathrm{d} \boldsymbol{r}
$$

Note that in MRI studies as described in sec. 2.1.5.2 the vector $\boldsymbol{k}$ will be proportional to the time $t$ and thus the inverse Fourier transform has been used here. Real space coordinates $\boldsymbol{r}$ are in units of meter $[\mathrm{m}]$ while the reciprocal space is its inverse and is given by $\boldsymbol{k}=(2 \pi)^{-1} \hat{\boldsymbol{e}}_{\boldsymbol{k}} / \lambda$, where $\lambda$ is the wavelength and $\boldsymbol{e}_{\boldsymbol{k}}$ is the unit vector in the direction of $\boldsymbol{k}$. Therefore, $\boldsymbol{k}$ is often called a wave vector and assumes values in units

of $\left[\mathrm{rad}^{-1} \mathrm{~m}^{-1}\right]$. The form factor $S_{0}(\boldsymbol{k})$ is in general a complex function and it would be 
desirable to measure this function directly.

Using imaging techniques such as MRI one measures the full reciprocal space or $\boldsymbol{k}$ space "signal" $S_{0}(\boldsymbol{k})$ and one can obtain the real space structural density $\rho_{0}(\boldsymbol{r})$ by Fourier transform (Callaghan (2011))

$$
\rho_{0}(\boldsymbol{r})=\int_{V_{k}} S_{0}(\boldsymbol{k}) \exp \{-\mathrm{i} 2 \pi \boldsymbol{k} \cdot \boldsymbol{r}\} \mathrm{d} \boldsymbol{k}
$$

The difference between imaging techniques and diffraction techniques lies in the ability of the former to measure the amplitude and the phase of the signal $S_{0}(\boldsymbol{k})$ and of the latter to measure only its intensity. The intensity is given by the absolute square of the signal $\left|S_{0}(\boldsymbol{k})\right|^{2}$. The shortcoming of diffraction techniques to measure only intensities is commonly known as the "phase problem" and prevents one from obtaining $\rho_{0}(\boldsymbol{r})$ directly by Fourier transform (Svergun et al. (1987)). Upon Fourier transform of $\left|S_{0}(k)\right|^{2}$ one obtains the pore density auto-correlation function

$$
\mathcal{F}\left\{\left|S_{0}(k)\right|^{2}\right\}=\rho_{0}^{*}(\boldsymbol{R})=\int_{V} \rho_{0}(\boldsymbol{r}+\boldsymbol{R}) \rho_{0}(\boldsymbol{r}) \mathrm{d} \boldsymbol{r},
$$

which contains less information about the pore structure than $\rho_{0}(\boldsymbol{r})$ itself. For brevity we denote the pore auto-correlation function with $\rho_{0}^{*}(\boldsymbol{R})$ in this work.

As will be shown in sec. 2.2.2 the signal amplitude of PGSE NMR is the Fourier transform of the average propagator $\bar{P}(\boldsymbol{R}, t)$ which was introduced in the previous section (eq. 1.3). Interestingly, the average propagator converges to the pore density autocorrelation function when the diffusion time is infinite $\bar{P}(\boldsymbol{R}, t \rightarrow \infty)=\rho_{0}^{*}(\boldsymbol{R})$.

As an illustrative example for the difference of imaging and diffraction techniques in general, consider a rectangular pore shape with a pore density function in one dimension given by

$$
\rho_{0}(z)= \begin{cases}\frac{1}{L} & \text { for }-L / 2 \leq z \leq L / 2 \\ 0 & \text { elsewhere }\end{cases}
$$

$S_{0}(k)$ for the rectangle which resembles a sinc function is shown in fig. 1.2 (a). With 
imaging techniques one obtains the form factor $S_{0}(k)$ including its phase which is either 0 or $\pi$ (negative as well as positive real part) for the rectangular shape. From the knowledge of $S_{0}(k)$ one can obtain the profile of the rectangular pore $\rho_{0}(z)$ directly by inverse Fourier transform as as shown in fig. $1.2(\mathrm{~b})$. When $\rho_{0}(r)$ is measured in $2 \mathrm{~d}$ it represents the $2 \mathrm{~d}$ image of the pore. In diffraction studies however, one measures
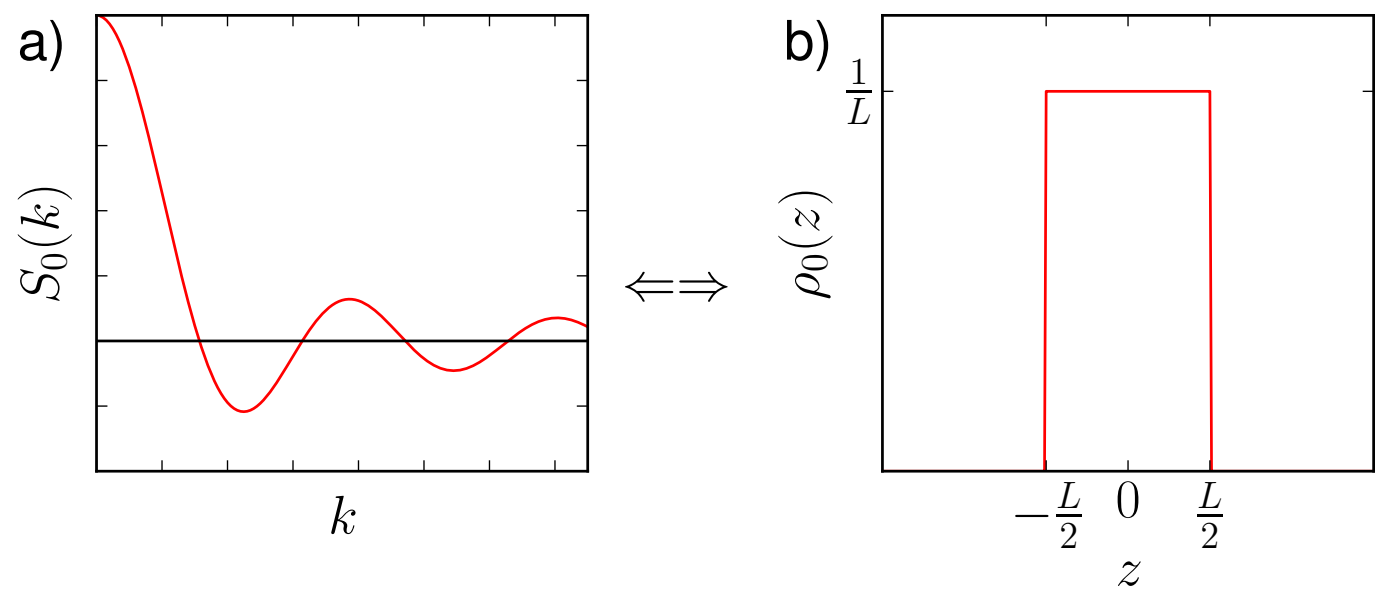

Figure 1.2: Form factor $S_{0}(k)$ for a rectangular pore as obtained by imaging methods such as MRI (a). Upon Fourier transform of $S_{0}(k)$ one obtains the pore density $\rho_{0}(z)$ projected on the direction of $k$, which represents a 1d image of the cross section of the pore (b).

the absolute square of the form factor $\left|S_{0}(k)\right|^{2}$ which lacks the phase information as shown in fig. 1.3 (a). The result upon Fourier transform yields the one dimensional auto-correlation function for the rectangular pore given by (Callaghan (2011))

$$
\mathcal{F}\left\{\left|S_{0}(k)\right|^{2}\right\}=\rho_{0}^{*}(Z)=\left\{\begin{array}{ccc}
\frac{L+Z}{L^{2}} & \text { for } & -L \leq Z \leq 0 \\
\frac{L-Z}{L^{2}} & \text { for } & 0<Z \leq L
\end{array}\right.
$$

which has a triangular shape as shown in fig. 1.3 (b). The pore density auto-correlation function $\rho_{0}^{*}(Z)$ still allows to extract information about the pore space such as its size, but the pore shape is obscured and one cannot obtain a $2 \mathrm{~d}$ image of the pore without further knowledge or assumptions.

The most commonly known example of diffraction phenomena is the double slit experiment also known as the Youngs interference experiment. In this experiment one 

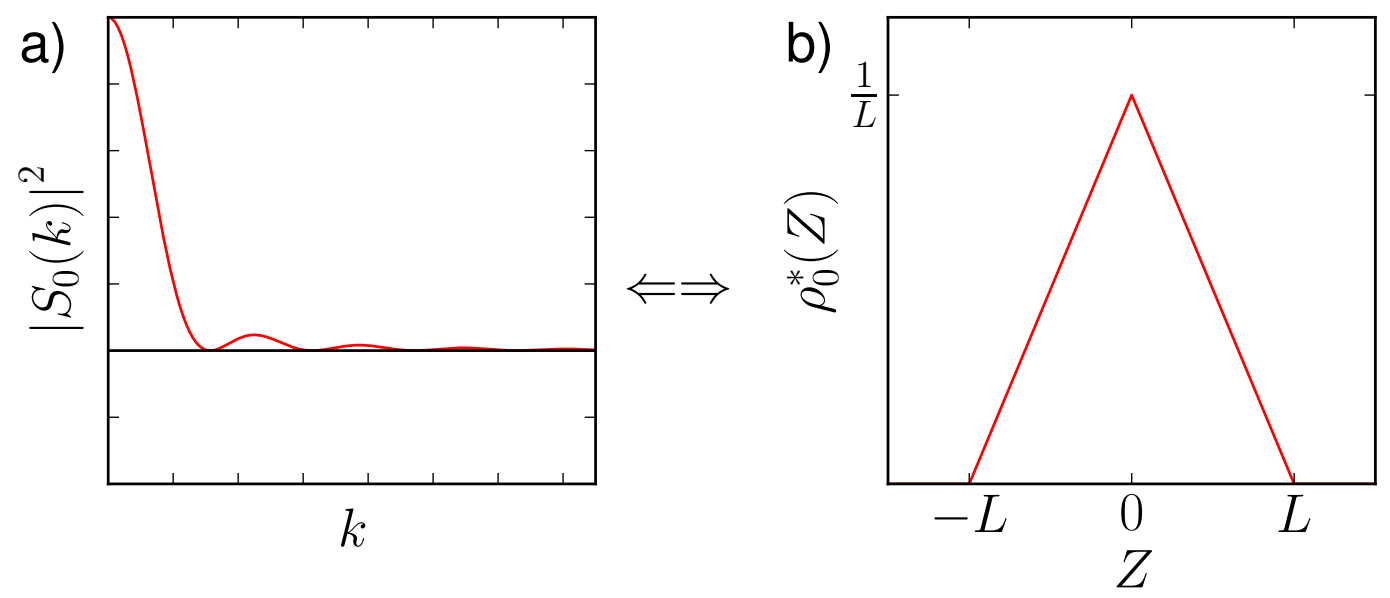

Figure 1.3: Absolute square of the form factor $\left|S_{0}(k)\right|^{2}$ for a rectangular pore as obtained by diffraction methods such as x-ray scattering and diffusive-diffraction PGSE NMR (a). Upon Fourier transform of $\left|S_{0}(k)\right|^{2}$ one obtains the pore density auto-correlation function $\rho_{0}^{*}(Z)$ projected on the direction of $k$. One may extract the size of the pore, but the pore shape is obscured. To reconstruct the pore image, one would need a priori assumptions about the underlying pore shape (b).

considers coherent light incident on a two slit system and observes the light intensity on a distant screen. A screen for visible light can only measure either light or dark regions and thus it is an energy-sensitive detector. The observed pattern is characterized by maxima and minima of the observed light intensity depending on the angle between the $\boldsymbol{k}$-vector and the slit system and thus the observed pattern describes the well known oscillations between bright and dark stripes which resemble $\left|S_{0}(k)\right|^{2}$ of the slit system.

In this work we present experimental proof of a further development of a diffraction method which allows us to measure the full complex form factor $S_{0}(k)$ and is therefore akin to an imaging technique. This advance allows one to obtain $2 \mathrm{~d}$ images of the average pore in the sample. 



\section{Theory and description of NMR diffusion techniques}

\subsection{NMR theory}

\subsubsection{Nuclear magnetization and rotating frame of reference}

In 1946 Felix Bloch in his famous paper "Nuclear Induction"(Bloch (1946)) laid out the basis for the technique that is today known as Nuclear Magnetic Resonance (NMR). Based on the work of Rabi et al. (1938), he found that magnetic moments of nuclei result in a nuclear polarization upon establishment of equilibrium in a static magnetic field. If this polarization is subject to radio frequency (rf) radiation at right angles to the constant field, it is forced to precess around the static field with decreasing latitude. This results in a component of nuclear polarization perpendicular to both the rf-field and the static field. This perpendicular component can induce an observable voltage in a coil.

The NMR technique relies on the presence of nuclei with a net nuclear spin. Spin is a property of particles arising from their intrinsic angular momentum and is associated with a magnetic moment $\mu$. In nuclei with unpaired nuclear spins there is a net nuclear spin characterized by the integer or half-integer number $I$ (Abragam (1961)). The magnetic moment associated with these nuclei is given by $\mu=\gamma \hbar I$, where $\gamma$ denotes the gyro-magnetic ratio and $\hbar$ is Plancks constant divided by $2 \pi$.

If an external magnetic field $\boldsymbol{B}_{0}$ is applied along the $z$-axis in the laboratory frame of reference the potential energy $\mathcal{H}_{\text {lab }}$ due to the Zeeman-interaction (Abragam (1961)) is 
given by

$$
\mathcal{H}_{\text {lab }}=-\gamma \hbar B_{0} m \quad m=I, I-1, \ldots,-I .
$$

$m$ denotes the projection of the net nuclear spin $I$ onto the direction of the applied magnetic field. For spin- $\frac{1}{2}$-systems such as ${ }^{1} \mathrm{H}$ and ${ }^{13} \mathrm{C}, m$ can only attain the two discrete values $m= \pm \frac{1}{2}$. In thermal equilibrium there is an excess occupation of the lower energy state $m=+\frac{1}{2}$ (Abragam (1961)), which is the state with spins pointing in the same direction as the magnetic field $B_{0}$. Since spin is leading to a magnetic moment, the occupation excess in the lower energy level leads to an excess of magnetic moments oriented along the applied magnetic field $\boldsymbol{B}_{0}$ and hence to a macroscopic nuclear magnetization $M_{0}$ of the sample given by

$$
\boldsymbol{M}_{0}=\left(\sum \mu_{+\frac{1}{2}}+\sum \mu_{-\frac{1}{2}}\right) \boldsymbol{e}_{z}
$$

One can show with the help of Boltzmanns law (Slichter (1996)) that the population excess between spins- $1 / 2$ aligned with the outer field $\left(N_{+}\right)$and opposing the outer field $\left(N_{-}\right)$in thermal equilibrium is given by

$$
\frac{N_{+}-N_{-}}{N_{+}+N_{-}}=\frac{1-\exp \left\{-\Delta E / k_{\mathrm{B}} T_{\mathrm{K}}\right\}}{1+\exp \left\{-\Delta E / k_{\mathrm{B}} T_{\mathrm{K}}\right\}} \approx \frac{\Delta E}{2 k T_{\mathrm{K}}}
$$

where $\Delta E$ is the energy difference between the two states $m= \pm 1 / 2$. In eq. 2.3 the high temperature approximation $k_{\mathrm{B}} T_{\mathrm{K}} \gg \Delta E$ and subsequent Taylor expansion truncated up to first order $\exp (X) \approx 1+X$ was used. For commonly used high-field superconducting magnets (9.4 Tesla) this population difference for ${ }^{1} \mathrm{H}$ nuclei and room temperature $T=$ $298 \mathrm{~K}$ is on the order of $10^{-5}$, meaning only ten in a million spins contribute to the signal. Note that the equilibrium magnetization $M_{0}$ is given by multiplying the population excess eq. 2.3 with the total number of nuclear spins $N$ times their magnetic moment $\mu=\gamma \hbar / 2$ yielding the Curie Law for $I=1 / 2$ spins

$$
M_{0}=N \frac{\gamma^{2} \hbar^{2} B_{0}}{4 k_{\mathrm{B}} T_{\mathrm{K}}}
$$


Each individual spin rotates about the direction of the applied magnetic field $\boldsymbol{B}_{0}$. Since the macroscopic magnetization $\boldsymbol{M}$ is the sum of the individual spins, it is also precessing around $\boldsymbol{B}_{0}$. Its precession angular frequency is called Larmor frequency which is given by

$$
\boldsymbol{\omega}_{0}=\gamma \boldsymbol{B}_{0}
$$

It corresponds to the frequency of an electromagnetic wave, which can induce transitions between the possible orientations of the nuclear spins in the external magnetic field.

If one follows the convention that the magnetic field is applied in $z$-direction then the observable signal is proportional to the $x$ - $y$-component of the magnetization $\boldsymbol{M}_{\mathrm{x}, \mathrm{y}}$ (Callaghan (1991)). Therefore, one has to disturb the spin system from its thermal equilibrium and rotate the magnetization into the $x$-y-plane. This can be achieved by applying an on-resonant $\left(\omega_{\mathrm{rf}}=\omega_{0}\right)$ radio-frequency-pulse (rf-pulse) perpendicular to the polarizing magnetic field (Bloch (1946)).

In order to allow for a coupling of the rf-pulse to the magnetization $\boldsymbol{M}_{0}$, one has to generate a rotating magnetic field $B_{1}$ precessing in the same direction and with the same angular frequency $\omega_{0}$ as the magnetization $\boldsymbol{M}_{0}$. From an engineering perspective it is possible to apply a linearly polarized oscillating magnetic field transverse to the $B_{0}$ field. This can be achieved by applying an alternating current to an rf-coil with its axis aligned transverse to the $B_{0}$ field as shown in fig. 2.1. The applied oscillating magnetic field with amplitude $2 B_{1} \cos \omega t$ can be represented by two counter-rotating circularly polarized fields with amplitude $B_{1}$. The part which is rotating in direction of the magnetization will couple to $\boldsymbol{M}_{0}$ and rotate it into the $x, y$-plane when the onresonance condition $\omega_{\mathrm{rf}}=\omega_{0}$ is fulfilled. The counter rotating component can be ignored if the condition $B_{1}<<B_{0}$ is satisfied (Callaghan (1991)).

After excitation one may utilize the rotating frame of reference as a useful tool to solve for the dynamics of the magnetization. In the laboratory frame of reference the Hamiltonian of non-interacting spins is given by adding a term arising from the $B_{1}$ 


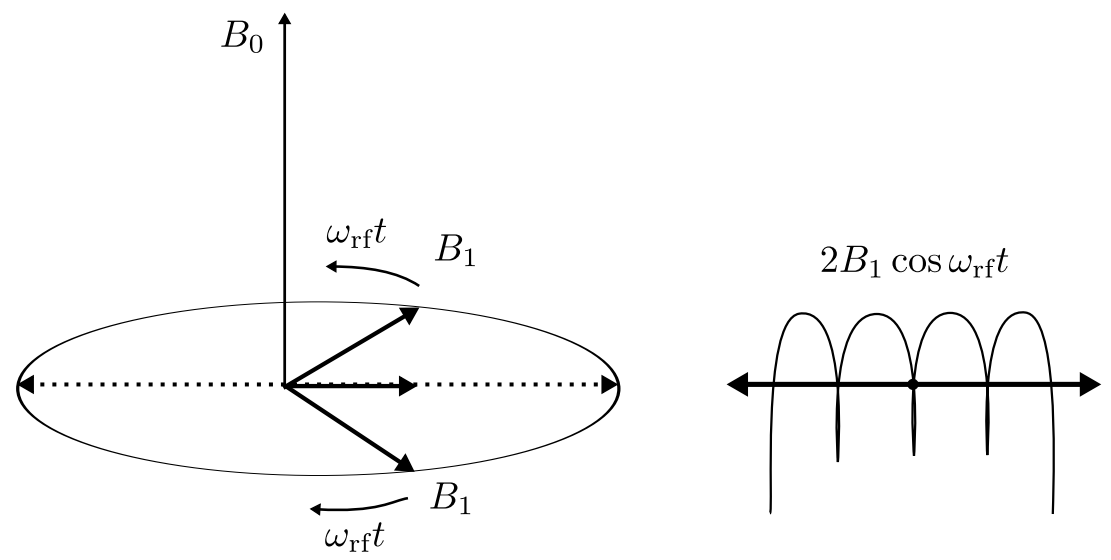

Figure 2.1: Application of an oscillating magnetic field through a solenoid perpendicular to the $B_{0}$ field. The linearly polarized field with amplitude $2 B_{1}$ can be represented as two counterrotating circularly polarized fields of amplitude $B_{1}$. Adapted from Callaghan (1991).

magnetic field to eq. 2.1 and is given by

$$
\mathcal{H}_{\text {lab }}=-\gamma B_{0} I_{\mathrm{z}}-2 \gamma B_{1} \cos \omega t I_{x}
$$

Since $\mathcal{H}_{\text {lab }}$ is time dependent it is hard to solve for the dynamics of the system directly. However, one may perform a coordinate transformation to a rotating frame of reference $x^{\prime}, y^{\prime}, z^{\prime}$, rotating at angular frequency $\omega$ (Rabi et al. (1954)). The precession around $B_{0}$ is reduced by the precession frequency of the rotating frame $\omega$. To account for this reduced influence of $B_{0}$ in the rotating frame of reference one has to subtract the field strength $\omega / \gamma$ from the laboratory reference frame Hamiltonian $H_{\text {lab }}$. Thus, the Hamiltonian in the rotating frame of reference is given by

$$
\mathcal{H}_{\mathrm{rot}}=-\gamma\left(B_{0}-\omega / \gamma\right) I_{\mathrm{z}}-\gamma B_{1} I_{x}
$$

On resonance $\left(\omega=\omega_{0}\right)$ the only magnetic field present in the rotating frame of reference is the rf-pulse magnetic field $B_{1}$. The magnetic field $B_{1}$ couples to the magnetization $M$ and rotates it about the direction of $B_{1}$ with the angular frequency $\omega_{1}=\gamma B_{1}$ as shown in fig. 2.2. The rotation angle depends on the length of application of the rf-pulse. Two rotation angles $\omega_{1} t$ are particularly often encountered in NMR experiments. First, the rotation of the magnetization into the $x$-y-plane, which corresponds to an angle of 


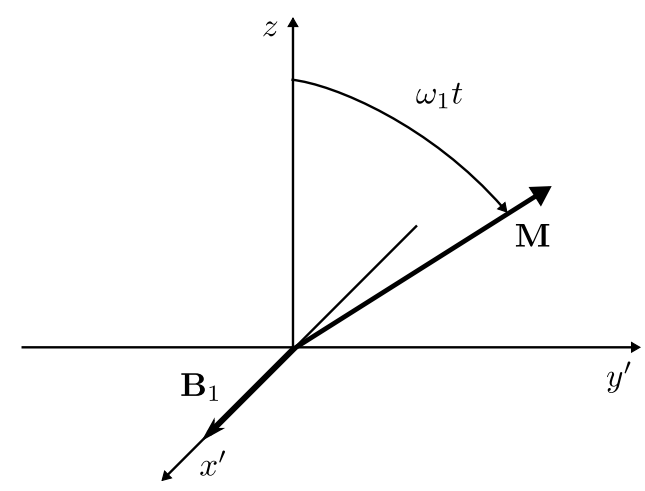

Figure 2.2: Apparent magnetic field $B_{1}$ in the rotating frame of reference and its influence on the magnetization $\boldsymbol{M}$. If the rf-magnetic field $B_{1}$ is applied on-resonance $\omega_{\mathrm{rf}}=\omega_{0}$ it rotates the magnetization into the $x, y$-plane. The angular frequency of rotation of the magnetization is $\omega_{1}$ and the direction of rotation is about the direction of $B_{1}$. Adapted from Callaghan (1991).

$\omega_{1} t=90^{\circ}$ and second the inversion of the magnetization corresponding to a rotation about $\omega_{1} t=180^{\circ}$.

\subsubsection{Relaxation}

Relaxation describes the tendency of a non-equilibrium spin system e.g. magnetization that is rotated in to the $x-y$ plane to return to thermal equilibrium $M_{0}$ along the $z$ direction. There are three major relaxation time constants affecting the magnetization after excitation (Bloembergen et al. (1948)). There is the spin-lattice relaxation characterized by the time constant $T_{1}$, the spin-spin relaxation or $T_{2}$-relaxation and there is loss of coherence of the magnetization components called $T_{2}^{*}$-relaxation. In general it holds that $T_{1} \geq T_{2} \geq T_{2}^{*}$ for reasons that will be examined below.

Any Rotation of the magnetization corresponds to an energetic excitation of the spin system, upon which the system tends to re-establish thermal equilibrium. This process involves the exchange of the potential energy of the spins with the surrounding thermal reservoir (or lattice). Thus, once the vector of magnetization $\boldsymbol{M}$ is rotated out of equilibrium it starts to re-align with the $z$-direction (Bloch (1946)). The time constant for this process is $T_{1}$ and it can be described by the differential equation

$$
\frac{\mathrm{d} M_{\mathrm{z}}}{\mathrm{d} t}=-\frac{\left(M_{\mathrm{z}}-M_{0}\right)}{T_{1}},
$$


for which one obtains upon integration

$$
M_{\mathrm{z}}(t)=M_{\mathrm{z}}(0) \exp \left\{\frac{-t}{T_{1}}\right\}+M_{0}\left(1-\exp \left\{\frac{-t}{T_{1}}\right\}\right)
$$

Therefore, $T_{1}$ relaxation describes the rate at which thermal equilibrium is re-established and gives an upper bound of experimental time of approximately $5 T_{1}$. After this time all elements of an NMR experiment including the acquisition have to be completed.

The interactions responsible for $T_{2}$ relaxation only involve the transverse components of the magnetization $M_{\mathrm{x}, \mathrm{y}}$. This so called spin-spin relaxation describes random jumps in the phases of spins caused by interactions in between spins as well as spin-lattice interactions. The random phase jumps lead to a decoherence of the transverse magnetization $M_{\mathrm{x}, \mathrm{y}}$ and to an irreversible loss of observable magnetization (Bloembergen et al. (1948)). The differential equation for this process is given by (Bloch (1946))

$$
\frac{\mathrm{d} M_{\mathrm{x}, \mathrm{y}}}{\mathrm{d} t}=-\frac{M_{\mathrm{x}, \mathrm{y}}}{T_{2}}
$$

which upon integration yields

$$
M_{\mathrm{x}, \mathrm{y}}(t)=M_{\mathrm{x}, \mathrm{y}}(0) \exp \left\{\frac{-t}{T_{2}}\right\}
$$

Note that $T_{2}$ relaxation does not involve any spin flips and hence the components of the magnetization that have undergone phase jumps still reside in the $x, y$-plane. Thus, these components are still subject to $T_{1}$ relaxation although they do not contribute to the measurable magnetization $M_{\mathrm{x}, \mathrm{y}}$. Hence, one may find that in NMR it holds that $T_{1} \geq T_{2}$.

$T_{2}^{*}$ relaxation on the other hand is caused by inhomogeneities of the polarizing magnetic field $B_{0}$. Such inhomogeneities may arise both from the design of the magnet system as well as from the sample itself. In this case the magnetic field $B_{0}$ has to be replaced by a local magnetic field $B_{0}^{\text {loc }}$, which is varying across the sample. One may divide the sample into volume elements located at $\boldsymbol{r}_{\mathrm{i}}$, where the nuclear spins in each volume element give rise to a magnetization density vector $\boldsymbol{m}_{\mathrm{i}}\left(\boldsymbol{r}_{\mathrm{i}}\right)$. The total magnetization of 
the sample is given by

$$
\boldsymbol{M}=\sum_{\mathrm{i}} \boldsymbol{m}_{\mathrm{i}}\left(\boldsymbol{r}_{\mathrm{i}}\right)
$$

Due to the spatial dependence of the magnetic field strength $B_{0}^{\text {loc }}$ one may find different Larmor frequencies in different volume elements of the sample. This leads to a successive build up of phase differences in between the magnetization density vectors $\boldsymbol{m}_{\mathrm{i}}$. Thus the magnetization $\boldsymbol{M}$, which is the vector sum (Eq. 2.12) of the magnetization density vectors $\boldsymbol{m}_{\mathrm{i}}$ is decaying due to this coherence loss. Consider for example the Free Induction Decay (FID) NMR experiment, which consists of a $90^{\circ}$-rf-pulse followed by the acquisition of the induced voltage in the rf-coil. Figure 2.3 (a) shows the magnetization $M_{\mathrm{x}^{\prime}, \mathrm{y}^{\prime}}$ just after the $90^{\circ} \mathrm{rf}-$ pulse in the rotating frame of reference $x^{\prime}, y^{\prime}$. Figure 2.3
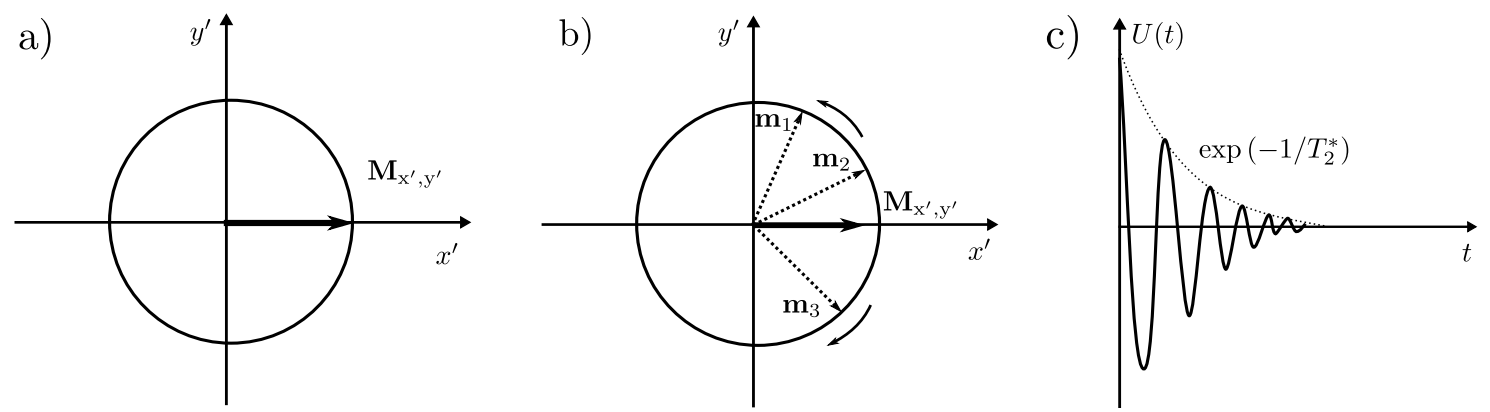

Figure 2.3: Schematic representation of the transverse magnetization just after a $90^{\circ} \mathrm{rf}-$ pulse in the rotating frame of reference $x^{\prime}, y^{\prime}$ (a). Transverse magnetization densities $\boldsymbol{m}_{1}, \boldsymbol{m}_{2}, \boldsymbol{m}_{3}$ dephasing through magnetic field inhomogeneities. The observable magnetization $M_{\mathrm{x}^{\prime}, \mathrm{y}^{\prime}}$ is given by vector addition of the magnetization density vectors from different parts of the sample (b). Induced voltage $U(t)$ of the transverse magnetization in the laboratory reference frame decaying with the relaxation rate $1 / T_{2}^{*}(\mathrm{c})$.

(b) shows schematically three magnetization density vectors $\boldsymbol{m}_{1}, \boldsymbol{m}_{2}, \boldsymbol{m}_{3}$ from different parts of the sample which are dephasing due to the locally different Larmor frequencies. The total magnetization $M_{\mathrm{x}^{\prime}, \mathrm{y}^{\prime}}$ is the vector addition of these magnetization vectors and thus after time $t$ its amplitude is reduced with respect to that in fig. 2.3 (a). Figure 2.3 (c) shows the decay of the induced voltage $U(t)$ in the laboratory reference frame in the rf-coil which decays proportional to $\exp \left\{-1 / T_{2}^{*}\right\}$. Note that all three relaxation processes, $T_{1}, T_{2}$ and $T_{2}^{*}$ relaxation, start to influence the magnetization just after the 
excitation pulse. However, the dephasing due to magnetic field inhomogeneities $T_{2}^{*}$ usually dominates (Bloembergen et al. (1948)). Thus if the time constants $T_{1}$ and $T_{2}$ shall be measured one may have to refocus the dephased magnetization using a train of refocusing rf-pulses (Carr and Purcell (1954)), which will be introduced in the next sections.

\subsubsection{Spin-echo pulse sequence}

The spin-echo pulse sequence was first proposed by Hahn (1950) and is now a basic building block of NMR experiments. Figure 2.4 shows the rf-pulse scheme of the spinecho experiment which consists of a leading $90^{\circ} \mathrm{rf}-$ pulse followed by a refocusing $180^{\circ}$ rf-pulse after a time period $\tau$. If this rf-pulse sequence is applied in an inhomogeneous

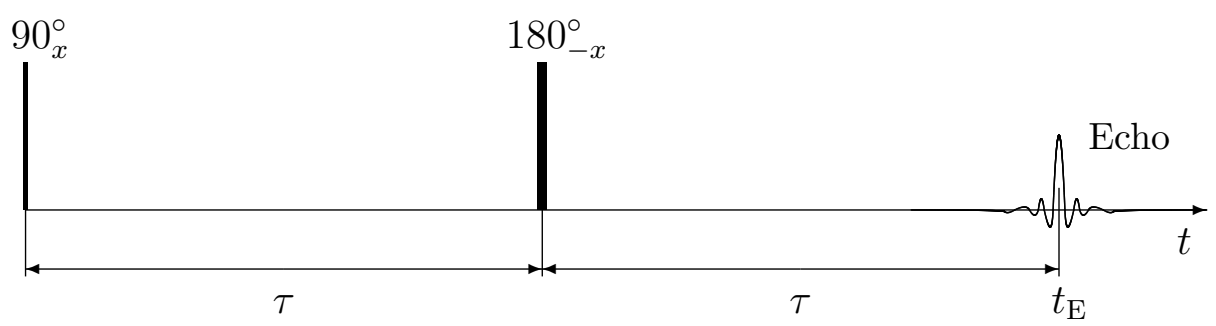

Figure 2.4: Spin-echo pulse sequence consisting of a leading $90^{\circ}$ rf-pulse which rotates the magnetization into the $x, y$-plane. It is followed by a $180^{\circ}$ rf-pulse after time $\tau$, which rotates the magnetization by $180^{\circ}$. At time $t_{\mathrm{E}}=2 \tau$ the acquired phase due to free precession in the inhomogeneous magnetic field is refocused, which leads to a spin-echo formation.

magnetic field a spin-echo forms at time $t_{\mathrm{E}}=2 \tau$. The spin echo can be understood by following the dephasing and rephasing of the magnetization density vectors $\boldsymbol{m}_{\mathrm{i}}$ from different volume elements in the sample. Figure 2.5 shows schematically the dynamics of three exemplary magnetization density vectors in the rotating frame of reference. After the $90^{\circ}$ excitation rf-pulse the magnetization $\boldsymbol{M}$ is rotated in the $x^{\prime}, y^{\prime}$-plane as shown in fig. 2.5 (a). Figure 2.5 (b) shows the magnetization after excitation where the individual magnetization vectors from different volume elements start to dephase due to their different Larmor frequencies. Thus, the observable magnetization decays with the relaxation rate $1 / T_{2}^{*}$ and one would be able to record an FID. Figure 2.5 (c) shows the 

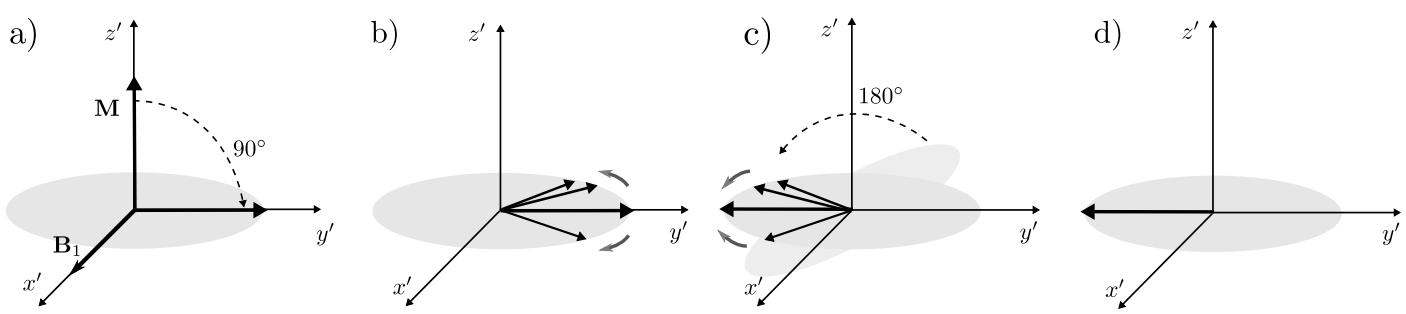

Figure 2.5: Scheme of the dynamics of the magnetization in the spin-echo experiment in the rotating frame of reference. The magnetization is rotated in the $x^{\prime}, y^{\prime}$-plane with a $90^{\circ} \mathrm{rf}-$ pulse (a). After excitation the magnetization vectors start to dephase due to different Larmor frequencies in different volume elements of the sample (b). The magnetization is rotated by $180^{\circ}$ by the second rf-pulse and starts to refocus (c). When the same time $\tau$ has elapsed as between the $90^{\circ}$ and $180^{\circ}$ rf-pulses the magnetization is re-focused and a spin-echo appears (d).

magnetization vectors just after application of the $180^{\circ} \mathrm{rf}-$ pulse. The rotation by $180^{\circ}$ inverts the acquired phase differences of each magnetization density vector $\boldsymbol{m}_{\mathrm{i}}$. Since the local Larmor frequencies do not change the acquired phase differences are starting to disappear. This leads to the refocusing of the magnetization at time $2 \tau$ after excitation as shown in fig. 2.5 (d). Note that the magnitude of the spin-echo is reduced in comparison to the FID through $T_{1}, T_{2}$ relaxation and self-diffusion of the spin-bearing particles in the in-homogeneous magnetic field (not shown). The spin-echo rf-pulse sequence is such a vital ingredient in NMR experiments since it extends the available time for studying the magnetization to $5 T_{2}$ instead of the much shorter time $5 T_{2}^{*}$. The relaxation rate $1 / T_{2}$ may be measured by repeatedly refocusing the magnetization as will be discussed in the next section.

\subsubsection{CPMG pulse sequence}

The Carr-Purcell-Meiboom-Gill (CPMG) pulse sequence as shown in fig. 2.6 (top) was introduced by Carr and Purcell (1954) and Meiboom and Gill (1958). It consists of a leading $90^{\circ} \mathrm{rf}$-pulse followed by a train of $180^{\circ} \mathrm{rf}$-pulses each separated by twice the initial $90^{\circ}-180^{\circ}$ rf-pulse separation $(2 \tau)$. Spin-echos form half way between the $180^{\circ}$ rf-pulses and decay with the transverse relaxation rate $1 / T_{2}$ (bottom). The acquisition of a train of spin-echos and the knowledge of the rf-pulse separation $2 \tau$ can therefore be utilized to measure the relaxation rate $1 / T_{2}$. However, in the presence of magnetic 


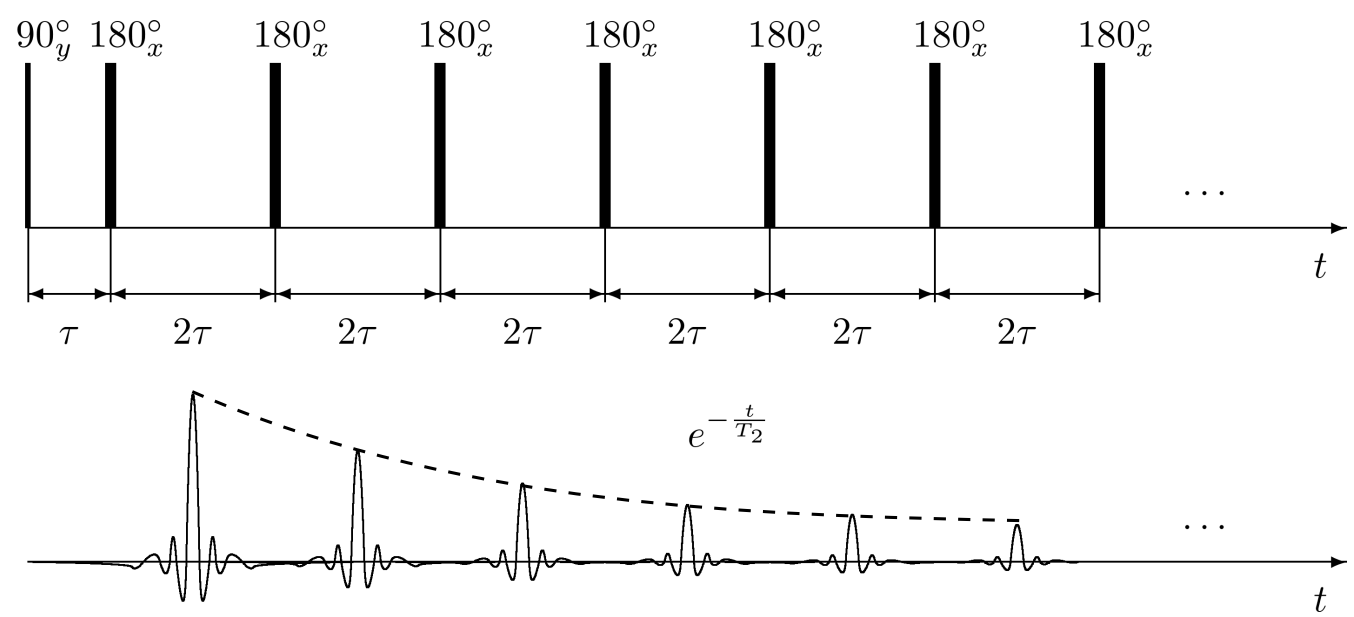

Figure 2.6: The CPMG rf-pulse sequence consisting of a leading $90^{\circ}$ rf-pulse followed by a train of $180^{\circ}$ rf-pulses each separated by twice the initial $90^{\circ}-180^{\circ}$ rf-pulse separation $2 \tau$ (top). Spin-echos form half way between the $180^{\circ}$ rf-pulses and decay with the transverse relaxation rate $1 / T_{2}$ (bottom).

field inhomogeneities this decay rate may be obscured by additional dephasing due to self-diffusion of the spin bearing molecules in a internal magnetic field gradient $g$ (a spatially dependent magnetic field as will be discussed in detail in sec. 2.1.5). Carr and Purcell (1954) derived the effective transverse relaxation rate given by

$$
\frac{1}{T_{2}^{\mathrm{eff}}}=\frac{1}{T_{2}}+\frac{1}{3} D(\gamma \boldsymbol{g} \tau)^{2}
$$

where $g$ be the magnitude of the magnetic field gradient and $D$ is the self-diffusion coefficient. The second term in eq. 2.13 can be minimized by reducing the rf-pulse separation $\tau$. Additionally, one may measure the relaxation rate $1 / T_{2}$ by performing a series of CPMG experiments with different times $\tau$ and extrapolating the $\tau$-dependent relaxation rate $1 / T_{2}^{\text {eff }}$ to $\tau=0$.

In addition to relaxation rate measurements, the CPMG rf-pulse sequence can be incorporated into other NMR experiments to exploit its special properties regarding magnetic field gradients (Callaghan and Stepisnik (1995)) and its influence on the phase coherence of the nuclear spin ensemble (Jenista et al. (2009), Álvarez et al. (2013)). In this work the CPMG rf-pulse sequence will be utilized as a building block of the MRPI 
pulse sequence as will be shown in sec. 3.1.1.

\subsubsection{Magnetic field gradients}

Magnetic field gradients are vital ingredients of MRI experiments (Bernstein et al. (2004)) and for NMR studies of translational motion (Callaghan (2011)). In this work they are utilized both for imaging and for the encoding of the self-diffusion process of spin bearing molecules. Magnetic field gradients can either be purposefully applied by specially built coils (Hidalgo-Tobon (2010)), or they are inherent in the experimental setup e.g. due to an inhomogeneous polarizing magnetic field $\boldsymbol{B}_{0}$ (Hürlimann (1998)).

Let the polarizing magnetic field point in $z$-direction $\boldsymbol{B}_{0}=\left(0,0, B_{0}\right)$. A magnetic field gradient $\boldsymbol{G}$ is given by

$$
\boldsymbol{G}=\left(G_{\mathrm{x}}, G_{\mathrm{y}}, G_{\mathrm{z}}\right)=\left(\frac{\partial B_{\mathrm{z}}}{\partial x}, \frac{\partial B_{\mathrm{z}}}{\partial y}, \frac{\partial B_{\mathrm{z}}}{\partial z}\right)
$$

According to eq. 2.14 the magnetic field gradient $\boldsymbol{G}$ may change the strength of the polarizing magnetic field $\boldsymbol{B}_{0}$, but not its direction. It has to be noted that transverse components which are not accounted for in eq. 2.14 are generated with every gradient since the magnetic field has to satisfy Maxwells equation $\nabla \cdot \boldsymbol{B}=0$. However, these so called concomitant fields are negligible under the condition that $|\boldsymbol{G} \cdot \boldsymbol{r}| \ll B_{0}$ (Bernstein et al. (1998)). This condition is usually satisfied for the gradient amplitudes and magnetic field strengths used in MRI and PGSE NMR applications (Bernstein et al. (1998)) although concomitant fields may be influential in low field systems (Volegov et al. (2005)).

If a magnetic field gradient is applied for a short time period $t$ it is called a pulsed gradient. A pulsed gradient $G(t)$ may be generated using a Maxwell coil design as shown in fig, 2.7 (Callaghan (2011)). In a Maxwell coil the loops are wound in such a way that the current $i(t)$ in one loop is flowing in the opposite direction as compared to the opposing loop. In the center of the gradient coil the generated magnetic fields cancel, while away from the center they enhance or subtract from the polarizing magnetic field. Pulsed gradients allow for a flexible design of PGSE NMR and MRI pulse sequences since 


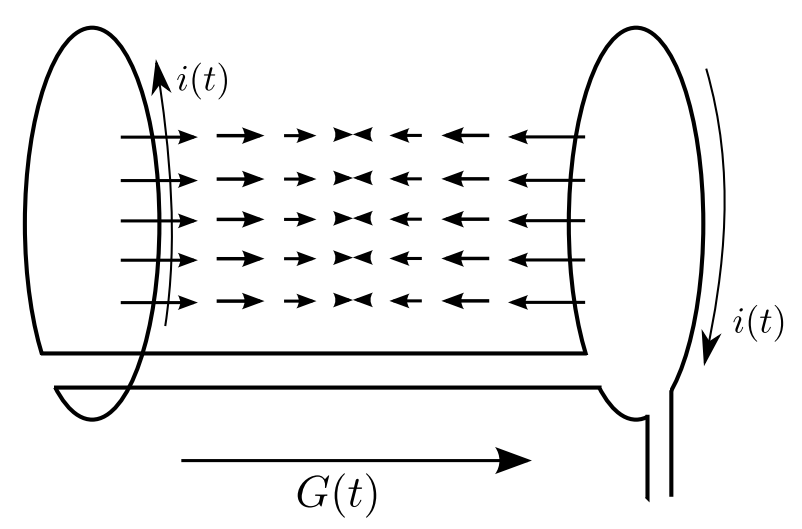

Figure 2.7: Scheme of a Maxwell coil generating a magnetic field gradient $G(t)$. The two loops are wound such that the current $i(t)$ in one loop is flowing in opposite direction as compared to the other loop. The lengths of the arrows along the axis of the coil indicate the local magnetic field strengths. The strength of the resulting magnetic field gradient $G(t)$ depends on the current $i(t)$ in the coil.

they can be applied for a well defined time period and their strength may be varied by the current amplitude $i(t)$ in the gradient coil.

\subsubsection{Effective magnetic field gradients}

The effect of the magnetic field gradients on the spin system can be reversed by application of a $180^{\circ}$ rf-pulse. However, it may be tedious to account for both the rf-pulse scheme and the magnetic field gradient scheme in case of many rf-pulses. Therefore, Mansfield and Pykett (1978) and Karlicek and Lowe (1980) introduced the concept of "effective" magnetic field gradients $\boldsymbol{G}^{*}(t)$ which allows to study the effect of magnetic field gradients on the spin system without having to account for rf-pulses.

As an example, consider the spin echo sequence as applied in a constant magnetic field gradient $\boldsymbol{G}$ as shown in fig. 2.8. In the laboratory frame of reference the magnetic field gradient $\boldsymbol{G}(t)$ stays constant with amplitude $\boldsymbol{G}$ throughout the experiment. However, the effective gradient $\boldsymbol{G}^{*}(t)$ as seen by the spin system changes sign when the $180^{\circ} \mathrm{rf}$ pulse is applied, since the effect of the gradient $\boldsymbol{G}$ on the spin system is inverted. The 


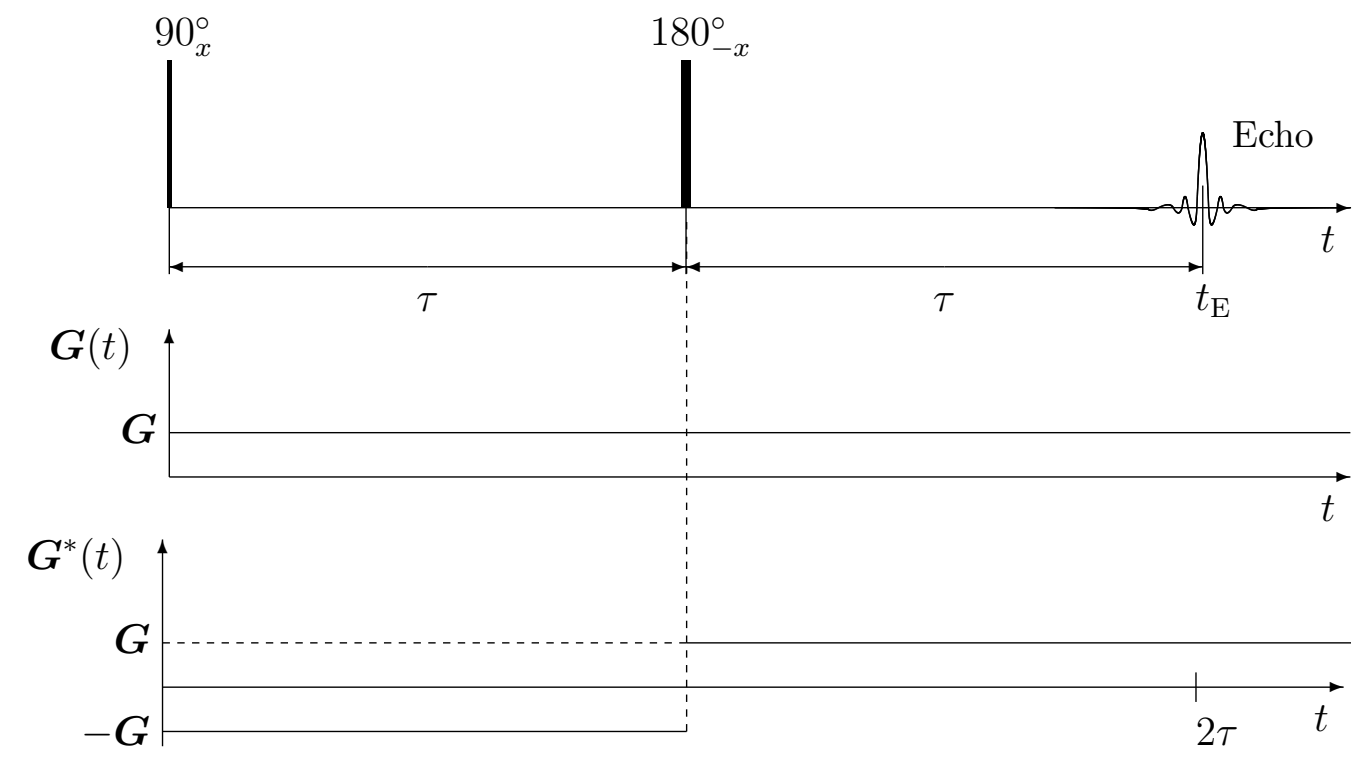

Figure 2.8: Spin echo pulse sequence under the influence of a constant gradient $G$. The effective magnetic field gradient $G^{*}(t)$ represents the temporal gradient scheme as seen by the spin system.

effective gradient $\boldsymbol{G}^{*}(t)$ is then given by

$$
\boldsymbol{G}(t) \rightarrow \boldsymbol{G}^{*}(t)= \begin{cases}-\boldsymbol{G} & \text { for } 0 \leq t \leq \tau \\ \boldsymbol{G} & \text { for } \tau<t\end{cases}
$$

In case of many $180^{\circ}$ rf-pulses one may start at the echo time $t_{\mathrm{E}}$ and work backwards, inverting the sign of the gradient $\boldsymbol{G}(t)$ each time a $180^{\circ}$ rf-pulse is encountered. The concept of effective gradients can be applied to static background gradients as well as pulsed gradients.

In order to obtain a spin echo at time $t_{\mathrm{E}}$ the total phase difference acquired during the gradient scheme has to be deleted. This requirement leads to the so called echo condition given by

$$
\int_{0}^{t_{\mathrm{E}}} G^{*}(t)=0 .
$$




\subsubsection{2 k-space}

Given the magnetic field gradient $\boldsymbol{G}$ the magnetic field at position $\boldsymbol{r}$ within the sample is given by $B(\boldsymbol{r})=B_{0}+\boldsymbol{G} \cdot \boldsymbol{r}$. This will lead to a spatially dependent Larmor frequency during application of the gradient given by

$$
\omega(\boldsymbol{r})=\gamma B_{0}+\gamma \boldsymbol{G} \cdot \boldsymbol{r} .
$$

This dependence of the Larmor frequency on the position $\boldsymbol{r}$ within the sample is the physical principle underlying the usefulness of magnetic field gradients, since one usually measures the frequencies and the associated phase shifts in MRI applications.

Consider a volume element $\mathrm{d} V$ at position $\boldsymbol{r}$ in the sample. The signal as detected by the rf-coil arising from this volume element is given by (Callaghan (1991))

$$
\begin{aligned}
\mathrm{d} S(\boldsymbol{G}, t) & =\rho(\boldsymbol{r}) \mathrm{d} V \exp \{\mathrm{i} \omega(\boldsymbol{r}) t\} \\
& =\rho(\boldsymbol{r}) \mathrm{d} V \exp \left\{\mathrm{i} \gamma B_{0}+\gamma \boldsymbol{G} \cdot \boldsymbol{r} t\right\},
\end{aligned}
$$

where $\rho(\boldsymbol{r})$ is the local spin density of the sample. In phase sensitive NMR detection the radio-frequency signal is mixed with a reference oscillation and the resulting signal is oscillating with the difference of both frequencies. If this mixing frequency is equal to $\gamma B_{0}$ the obtained signal is oscillating with $\gamma \boldsymbol{G} \cdot \boldsymbol{r}$. Thus, one may neglect the $\gamma B_{0}$ term in eq. 2.18. The integrated signal amplitude is given by

$$
S(\boldsymbol{G}, t)=\int_{V} \rho(\boldsymbol{r}) \exp \{\mathrm{i} \gamma \boldsymbol{G} \cdot \boldsymbol{r} t\} \mathrm{d} \boldsymbol{r}
$$

where $\mathrm{d} \boldsymbol{r}$ represents the volume integration. This relation is similar to the Fourier relationship already found in sec. 1.3 and which is given by Eq. 1.9. Mansfield and Pykett (1978) introduced the concept of $\boldsymbol{k}$-space by defining the reciprocal vector $\boldsymbol{k}$ according to

$$
\boldsymbol{k}=(2 \pi)^{-1} \gamma \boldsymbol{G} t
$$


This definition shows that $\boldsymbol{k}$-space may be traversed by advancing either in time $t$ or by changing the gradient amplitude. The direction of this traverse is determined by the direction of the gradient $\boldsymbol{G}$. Substituting $\boldsymbol{k}$ in eq. 2.20 one obtains the Fourier relationship and its inverse given by

$$
\begin{aligned}
& S(\boldsymbol{k})=\int_{V} \rho(\boldsymbol{r}) \exp \{\mathrm{i} 2 \pi \boldsymbol{k} \cdot \boldsymbol{r}\} \mathrm{d} \boldsymbol{r} \\
& \rho(\boldsymbol{r})=\int_{V_{k}} S(\boldsymbol{k}) \exp \{-\mathrm{i} 2 \pi \boldsymbol{k} \cdot \boldsymbol{r}\} \mathrm{d} \boldsymbol{k},
\end{aligned}
$$

where $V_{k}$ denotes the volume of $\boldsymbol{k}$-space. Note that this Fourier pair is similar to eq. 1.9 and eq. 1.10, the difference being that the subscript zero was dropped, since here the spin density does not represent that of a single pore, but can be any distribution related to the shape and spin density of the sample. Furthermore, since the signal will be sampled at discrete time steps, the Fourier transform of $S(\boldsymbol{k})$ will not return the original function $\rho(\boldsymbol{r})$, but a discrete representation.

\subsubsection{3 q-space}

The concept of $q$-space is employed if two compensating pulsed gradients are applied (Callaghan (1991)). If such a gradient pair is used in conjunction with a spin echo pulse sequence (sec. 2.1.3) one is able to measure phase shifts related to the molecular motion of the spin bearing molecules. This way the mean squared displacement of the molecules can be measured, which will be discussed in detail in sec. 2.2.1.

Here, the focus lies on the conceptual difference between two compensating gradient pulses and the phase shift arising from molecular motion ( $\boldsymbol{q}$-space) in contrast to a phase shift due to the location of the spins ( $\boldsymbol{k}$-space). For this purpose, consider the gradient pulses to be sufficiently narrow such that the motion during the pulses can be neglected. This so called "narrow-pulse approximation" is equal to saying that the gradient pulse duration $\delta$ is much smaller that the gradient pulse separation $\Delta$, i.e. $\delta \ll \Delta$ (Callaghan (1991)).

The effect of the first gradient is to imprint a phase shift of $\gamma \delta \boldsymbol{G} \cdot \boldsymbol{r}_{0}$ on a spin located 
at position $\boldsymbol{r}_{0}$. Using a spin-echo sequence the $180^{\circ} \mathrm{rf}-$ pulse subsequently inverts this phase. Suppose that the spin bearing molecule has moved to position $\boldsymbol{r}$ before the second gradient pulse. The phase shift after the second pulse is given by $\gamma \delta \boldsymbol{G} \cdot\left(\boldsymbol{r}-\boldsymbol{r}_{0}\right)$. Let $S(\boldsymbol{G}, \Delta)$ be the amplitude of the spin-echo at its center and for brevity we neglect relaxation influences. The signal amplitude $S(\boldsymbol{G}, \Delta)$ is given by the integral over all transverse magnetizations carrying the phase term $\exp \left\{\mathrm{i} \gamma \delta \boldsymbol{G} \cdot\left(\boldsymbol{r}-\boldsymbol{r}_{0}\right)\right\}$. This term has to be weighted by the probability density for a spin to start at position $\boldsymbol{r}_{0}$ and to move to $\boldsymbol{r}$. This probability density is equal to $\rho\left(\boldsymbol{r}_{0}\right) P\left(\boldsymbol{r}_{0}, \boldsymbol{r}, \Delta\right)$, as discussed in sec. 1.2 where we have replaced the probability density $p\left(\boldsymbol{r}_{0}\right)$ by the initial spin density $\rho\left(\boldsymbol{r}_{0}\right)$. The total signal is given by

$$
S(\boldsymbol{G}, \Delta)=\int_{V} \rho\left(\boldsymbol{r}_{0}\right) \int_{V} P\left(\boldsymbol{r}, \boldsymbol{r}^{\prime}, \Delta\right) \exp \left\{\mathrm{i} \gamma \delta \boldsymbol{G} \cdot\left(\boldsymbol{r}-\boldsymbol{r}_{0}\right)\right\} \mathrm{d} \boldsymbol{r} \mathrm{d} \boldsymbol{r}_{0}
$$

which is similar to eq. 2.20. Analogous to $\boldsymbol{k}$-space one may define a reciprocal space vector given by

$$
\boldsymbol{q}=(2 \pi)^{-1} \gamma \delta \boldsymbol{G}
$$

and eq. 2.24 can be rewritten

$$
S(\boldsymbol{q}, \Delta)=\int_{V} \rho\left(\boldsymbol{r}_{0}\right) \int_{V} P\left(\boldsymbol{r}_{0}, \boldsymbol{r}, \Delta\right) \exp \left\{\mathrm{i} 2 \pi \boldsymbol{q} \cdot\left(\boldsymbol{r}^{\prime}-\boldsymbol{r}\right)\right\} \mathrm{d} \boldsymbol{r} \mathrm{d} \boldsymbol{r}_{0}
$$

This equation is similar to the scattering function which is measured in quasi-elastic neutron scattering (Jobic (2000)) and dynamic light scattering (Berne and Pecora (2000)). Most notably the technique is analog to scattering techniques in general where the gradient pulses resemble the incoming and outgoing wave-vectors with $\boldsymbol{q}$ being the scattering wave-vector.

Furthermore, making the substitution $\boldsymbol{r}=\boldsymbol{r}_{0}+\boldsymbol{R}$ in eq. 2.26 it may be rewritten and 
one obtains the Fourier pair

$$
\begin{aligned}
& S(\boldsymbol{q}, \Delta)=\int_{V} \bar{P}(\boldsymbol{R}, \Delta) \exp \{\mathrm{i} 2 \pi \boldsymbol{q} \cdot \boldsymbol{R}\} \mathrm{d} \boldsymbol{R} \\
& \bar{P}(\boldsymbol{R}, \Delta)=\int_{V_{q}} S(\boldsymbol{q}, \Delta) \exp \{-\mathrm{i} 2 \pi \boldsymbol{q} \cdot \boldsymbol{R}\} \mathrm{d} \boldsymbol{q},
\end{aligned}
$$

where $\bar{P}(\boldsymbol{R}, \Delta)$ is the average propagator found in sec. 1.2 and $V_{q}$ is the volume of $\boldsymbol{q}$-space. Thus, acquisition of the signal in $\boldsymbol{q}$-space permits the imaging of $\bar{P}(\boldsymbol{R}, \Delta)$ by labeling for displacements just as acquisition in $\boldsymbol{k}$-space yields an image of $\rho(\boldsymbol{r})$ by labeling for positions.

This important relation is the basis of many PGSE NMR techniques measuring translational motion (Callaghan (2011)). Of special relevance for this work is the case of restrictions to the displacement e.g. due to the walls of a pore. In this case the average propagator may contain information about the confining domain via the expansion in eigenfunctions of the confining domain (eq. 1.8) as will be further explored in sec. 2.2.2.

\subsection{NMR diffusive-diffraction techniques}

\subsubsection{Pulsed Gradient Spin Echo (PGSE) NMR}

The earliest NMR diffusion experiments were carried out using the spin-echo pulse sequence developed by Hahn (1950). As in the works of Carr and Purcell (1954) and Douglass and McCall (1958) the required magnetic field gradient $G$ was kept constant during the entire time of the experiment and the spin-echo attenuation was studied by varying the time between the $90^{\circ} \mathrm{rf}$-pulse and the spin-echo $(2 \tau)$. Woessner $(1960)$ showed that in the case of restrictions to the self-diffusion of the molecules the measured self-diffusion coefficient depends on the time $\tau$ and thus the method of varying this time was prone to misinterpretation. In the same work Woessner (1960) proposed to keep the time $2 \tau$ constant and instead vary the gradient strength $G$ by changing the electric current through the gradient coil system. Employing this concept Woessner (1963) could show experimentally that a time-dependent apparent self-diffusion coefficient as 
measured by NMR $D_{\text {app }}$ can be defined. $D_{\text {app }}$ is influenced by boundaries such as pore walls which are limiting the mean-square displacement of the diffusing molecules and thus $D_{\text {app }}$ may vary considerably from the bulk self-diffusion coefficient $D$. However, this method introduced new problems such as the change in the selectivity of the rf-pulses with gradient strength $G$. Therefore, Stejskal and Tanner (1965) introduced the use of time dependent pulsed field gradients which are switched on for a short time period $\delta$ in between the rf-pulses. The pulsed gradient spin echo (PGSE) NMR sequence shown in fig. 2.9 not only overcame the problem of rf-pulse selectivity, but also introduced a well defined observation time $\Delta$, which extends from the start of the first gradient pulse to the start of the second gradient pulse assuming $\delta$ is short compared to $\Delta$. As discussed

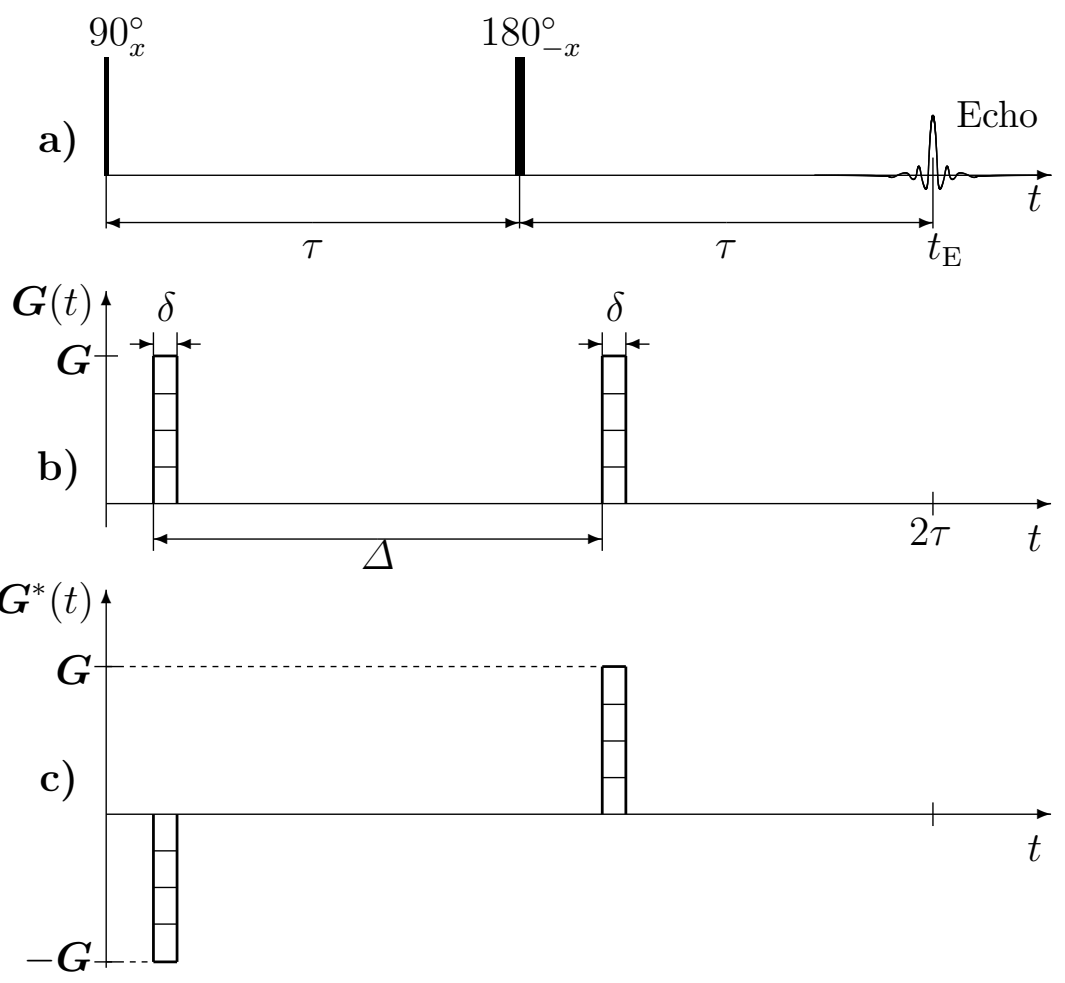

Figure 2.9: The pulsed gradient spin echo sequence as proposed by Stejskal and Tanner (1965). The time period between the two first rf-pulses is denoted by $\tau$ and a spin echo forms at time $2 \tau$ (a). Two magnetic field gradient pulses of variable amplitude $G$ and duration $\delta$ are applied in between the rf pulses with a time separation $\Delta$ separating their leading edges (b). The effective gradient pulses as seen by the spin system appear with opposite polarity due to the phase inversion by the $180^{\circ} \mathrm{rf}$ pulse (c). The normalized spin echo amplitude $E$ is primarily sensitive to the mean squared displacement due to self-diffusion during the observation time $\Delta$. 
in sec. 2.1.5.2, during application of a magnetic field gradient pulse of amplitude $\boldsymbol{G}$ the magnetic field $\boldsymbol{B}(r)$ depends on the spatial coordinate $\boldsymbol{r}$. If one defines the direction of $B_{0}$ to be the $z$-direction and the magnetic field gradient is applied along $B_{0}$ then the magnetic field is given by adapting eq. 2.17 to yield

$$
B(z, t)=B_{0}+G_{z}(t) z
$$

At different positions $z$ in the sample the spins rotate with angular frequencies $\omega_{0}(z)$ according to the Larmor equation

$$
\omega_{0}(z)=\gamma B_{0}+\gamma G_{z} z
$$

This space dependent angular frequency is only present during the gradient pulse application time $\delta$. After this time $\delta$ there is a space dependent phase shift of the nuclear spins along the $z$-axis. Since the $180^{\circ}$ rf-pulse inverts the effect of the magnetic field gradient on the accumulated magnetization phase, the effective gradient is given for the PGSE NMR sequence by

$$
\boldsymbol{G}^{*}(t)=\left\{\begin{array}{lll}
-\boldsymbol{G}(t) & \text { for } & t \leq \tau \\
+\boldsymbol{G}(t) & \text { for } & t>\tau
\end{array}\right.
$$

The time dependence of $G^{*}(t)$ is shown in fig. 2.9 (c). This allows to refocus the spins with a second pulsed field gradient if the spins with the respective phases remained at their places. If the second gradient has the same amplitude $G$ and duration $\delta$, the space dependent phase shift is deleted and the maximum magnetization is refocused in a spin echo at time $t_{\mathrm{E}}=2 \tau$.

If on the other hand, a molecule changed its z-position due to self-diffusion during the observation time $\Delta$, it carries its spin and hence the phase shift with it. Self-diffusion then leads to a redistribution of the phase shifts in space and thus to an incomplete refocusing through the second gradient pulse. The spin echo amplitude is therefore primarily sensitive to the mean squared displacement $\left\langle z^{2}\right\rangle=2 D \Delta$ of the spin-bearing 
particles during the observation time $\Delta$.

PGSE NMR diffusion experiments are performed by varying the gradient strength $G$ and recording the resulting spin echo amplitude $S(G, \Delta)$. For an experiment with all times fixed but only the gradient strength varied, relaxation leads to a constant attenuation factor which cancels when normalizing to $E(G, \Delta)=S(G, \Delta) / S(0, \Delta)$. In the PGSE NMR diffusion literature as reviewed by Stallmach and Galvosas (2007) the influence of the gradient duration and amplitude is often summed up in one factor, commonly referred to as $b$-value. The expression for the normalized spin echo amplitude can then be expressed as

$$
E(b)=\exp \{-b D\}
$$

The influence of gradients on the spin echo amplitude can be calculated with the double time integral of the effective pulsed field gradients $G^{*}(t)$

$$
b=\gamma^{2} \int_{0}^{t} \mathrm{~d} t^{\prime}\left[\int_{0}^{t^{\prime}} \mathrm{d} t^{\prime \prime} \boldsymbol{G}^{*}\left(t^{\prime \prime}\right)\right]^{2}
$$

Evaluation of the integrals in eq. 2.33 for a pair of rectangular gradient pulses as schematically shown for $G^{*}(t)$ in fig. 2.9 yields (Stejskal and Tanner (1965))

$$
E(G, \Delta)=\exp \left\{-D \gamma^{2} \delta^{2}\left(\Delta-\frac{1}{3} \delta\right) G^{2}\right\}
$$

Using this pulse sequence, Stejskal (1965) studied diffusion in restricted systems and a multitude of studies followed (Kärger et al. (1988)). NMR diffusion experiments offer insight into the micro-structure of a sample below the resolution limit of NMR microimaging techniques. The vast literature on the influence of reflecting boundaries such as pores on NMR diffusion experiments has recently been reviewed by Grebenkov (2007). One particularly striking result was obtained by Callaghan et al. (1991), for the case that the observation time $\Delta$ is increased such that $D \Delta \gg L^{2}$, where $L$ is the size of a restriction such as a closed pore radius. In this case the normalized signal dependence 
on the gradient amplitude resembles a diffractogram of the pore space, which allowed to obtain detailed information about porous media structures as discussed in the next section.

\subsubsection{Diffusive-diffraction PGSE NMR}

Cory and Garroway (1990) showed on the example of water diffusing in yeast cells that compartmentalization of a sample can be detected with PGSE NMR diffusion studies. This was soon followed by the theoretical prediction (Callaghan (1990)) and later experimental proof of diffusive-diffraction PGSE NMR by Callaghan et al. (1991). The name diffusive-diffraction PGSE NMR was chosen, because the technique shows close resemblance to light, x-ray and neutron scattering experiments (Mansfield and Grannell (1975), Cotts (1991), Barrall et al. (1992)). Analogous to diffraction techniques, in diffusive-diffraction PGSE NMR one defines the wave vector $\boldsymbol{q}=(2 \pi)^{-1} \gamma \boldsymbol{G} \delta$ (see sec. 2.1.5.3), which resembles the change between the incoming and outgoing waves in scattering experiments.

To derive the governing expressions of diffusive-diffraction PGSE NMR one may consider the case of a single closed pore. Figure 2.10 shows schematically the pore spin density $\rho_{0}(\boldsymbol{r})$ of a closed pore with circular cross section of radius $L . \rho_{0}(\boldsymbol{r})$ is assumed to be equal to one inside the pore and zero outside. In the previous section in eq. 2.34 the

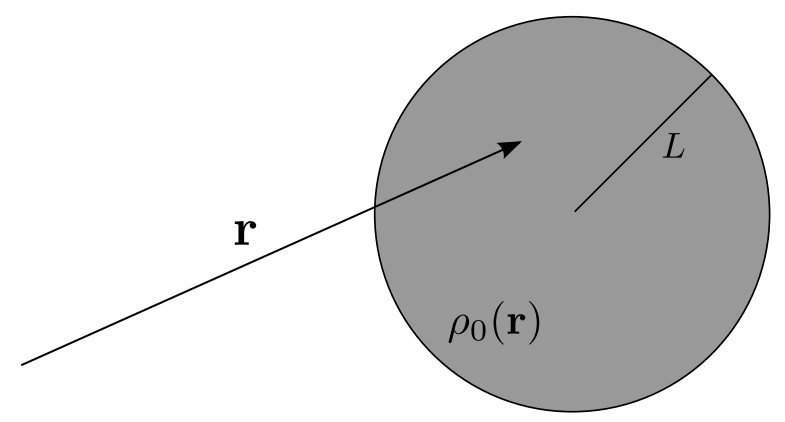

Figure 2.10: Sketch of the pore spin density of an isolated closed pore. If the pore is filled with a homogeneous fluid the density is constant within the pore and zero outside.

normalized spin-echo amplitude $E(G, \Delta)$ was given for the case of unrestricted Gaussian self-diffusion. In case of restrictions to the molecular self-diffusion one has to evaluate 
a more general expression given by the ensemble average of the phase $\Phi$ that the spins acquire during the NMR diffusion experiment (Douglass and McCall (1958), Neuman (1974))

$$
E=\langle\exp \{-\mathrm{i} \Phi\}\rangle
$$

The ensemble average $\langle\ldots\rangle$ has to be taken over all spins contributing to the signal formation and can be replaced by the volume integral over the spin density function $\langle\ldots\rangle=1 / V \int_{V} \ldots \rho_{0}(\boldsymbol{r}) \mathrm{d} \boldsymbol{r}$. In this analysis the phase $\Phi$ contains contributions due to the Brownian motion of the spin bearing molecules in a spatially and temporally varying magnetic field $B(\boldsymbol{r}, t)$. Similar to the previous analysis in sec. 2.2.1, the static polarizing magnetic field $B_{0}$ can be superimposed by a pulsed field gradient $\boldsymbol{G}^{*}(t)$ (Stejskal and Tanner (1965)), such that the magnetic field at time $t$ at position $\boldsymbol{r}(t)$ is given by $B(\boldsymbol{r}, t)=B_{0}+\boldsymbol{G}^{*}(t) \cdot \boldsymbol{r}(t)$. Suppose that the gradient pulse pattern is of total duration $T$ and the rephasing- or echo-condition $\int_{0}^{T} \boldsymbol{G}^{*}(t) \mathrm{d} t=0$ is fulfilled.

A spin following a path described by the random variable $\boldsymbol{r}(t)$ acquires a phase $\Phi_{\delta}=\gamma \int_{0}^{\delta} \boldsymbol{G}^{*}(t) \cdot \boldsymbol{r}(t) \mathrm{d} t$ during the time interval $\delta$ under the influence of a magnetic field gradient of amplitude $\boldsymbol{G}^{*}(t)$. Assuming a constant gradient $\boldsymbol{G}$ during this time interval this relation can be rewritten as

$$
\Phi_{\delta}=2 \pi \boldsymbol{q} \cdot \frac{1}{\delta} \int_{0}^{\delta} \boldsymbol{r}(t) \mathrm{d} t
$$

Diffusive-diffractive PGSE NMR consists of two magnetic field gradient pulses of duration $\delta$ with opposite polarity separated by the time $\Delta$ as shown for the effective gradient pattern $G^{*}(t)$ in fig. 2.9. Equation 2.36 can be modified to resemble the situation of the diffusive-diffractive PGSE NMR experiment by integrating over the whole time $T$ of the experiment. Since the magnetic field gradient is only non-zero during the two short pulses of duration $\delta$ one arrives at

$$
\Phi_{\Delta, \delta}=2 \pi \boldsymbol{q} \cdot\left[\frac{1}{\delta} \int_{\Delta}^{\Delta+\delta} \boldsymbol{r}_{1}(t) \mathrm{d} t-\frac{1}{\delta} \int_{0}^{\delta} \boldsymbol{r}_{0}(t) \mathrm{d} t\right]
$$


Here, $\boldsymbol{r}_{1}(t)$ and $\boldsymbol{r}_{0}(t)$ are the positions of a spin during the two gradient pulses. Generally, $\boldsymbol{r}_{1}(t)$ and $\boldsymbol{r}_{0}(t)$ are random variables since the spins undergo Brownian motion in the pore space. Equation 2.37 can be significantly simplified in the limit of the narrow pulse approximation. As mentioned in sec. 2.1.5.3, this approximation assumes that the pulsed field gradient is short such that $\delta \rightarrow 0$ while the area $G \delta$ is kept constant. In this case the time integrals can be replaced by the instantaneous positions at the times of the gradient pulses $\boldsymbol{r}_{0}(0)$ and $\boldsymbol{r}_{1}(\Delta)$ leading to

$$
\Phi_{\Delta, \delta \rightarrow 0}=2 \pi \boldsymbol{q} \cdot\left[\boldsymbol{r}_{1}(\Delta)-\boldsymbol{r}_{0}(0)\right]
$$

This expression has to be inserted into eq. 2.35 and in order to obtain the NMR signal amplitude one has to average over the ensemble of spins

$$
E_{\Delta}(\boldsymbol{q})=\left\langle\exp \left\{-\mathrm{i} 2 \pi q \cdot\left[\boldsymbol{r}_{1}(\Delta)-\boldsymbol{r}_{0}(0)\right]\right\}\right\rangle
$$

If the gradient separation time is infinitely large $\Delta \rightarrow \infty$ such that

$$
D \Delta \gg L^{2}
$$

the starting $\boldsymbol{r}_{0}(0)$ and final positions $\boldsymbol{r}_{1}(\Delta)$ lose their correlation due to their multiple reflections at the pore walls. Thus, on average the molecules have to traverse the pore space multiple times during the observation time $\Delta$. If this condition is fulfilled, the ensemble average can be calculated for each exponential separately leading to

$$
\begin{aligned}
E_{\infty}(\boldsymbol{q}) & =\left\langle\exp \left\{-\mathrm{i} 2 \pi \boldsymbol{q} \cdot \boldsymbol{r}_{1}\right\}\right\rangle\left\langle\exp \left\{\mathrm{i} 2 \pi \boldsymbol{q} \cdot \boldsymbol{r}_{0}\right\}\right\rangle \\
& =\int_{V} \rho_{0}(\boldsymbol{r}) \exp \left\{-\mathrm{i} 2 \pi \boldsymbol{q} \cdot \boldsymbol{r}_{1}\right\} \mathrm{d} \boldsymbol{r} \int_{V} \rho_{0}(\boldsymbol{r}) \exp \left\{\mathrm{i} 2 \pi \boldsymbol{q} \cdot \boldsymbol{r}_{0}\right\} \mathrm{d} \boldsymbol{r} .
\end{aligned}
$$

Using the definition of the form factor $S_{0}(\boldsymbol{q})=\int_{V} \rho_{0}(\boldsymbol{r}) \mathrm{e}^{-\mathrm{i} 2 \pi \boldsymbol{q} \cdot \boldsymbol{r}} \mathrm{d} \boldsymbol{r}$ given by eq. 1.9 one 
obtains

$$
\begin{aligned}
E_{\infty}(\boldsymbol{q}) & =S_{0}(\boldsymbol{q}) S_{0}^{*}(\boldsymbol{q}) \\
& =\left|S_{0}(\boldsymbol{q})\right|^{2}
\end{aligned}
$$

Thus, this experiment yields the absolute square of the form factor. Figure 2.11 shows the simulated PGSE NMR signal $E(q)$ on the example of cylindrical pores for the ratios $D \Delta / L^{2}=0.2$ (squares), $D \Delta / L^{2}=0.5$ (triangles), $D \Delta / L^{2}=1.0$ (circles) and $D \Delta / L^{2}=2.0$ (diamonds). With increasing ratio the signal converges to the absolute square of the form factor of the cylindrical pore $\left|S_{0}\right|^{2}=\left|J_{1}(2 \pi \boldsymbol{q} L) /(\pi \boldsymbol{q} L)\right|^{2}$ (solid line). Note that only positive values can be measured just as it is common for diffraction techniques and the phase information is lost as discussed in sec. 1.3. For the case of a

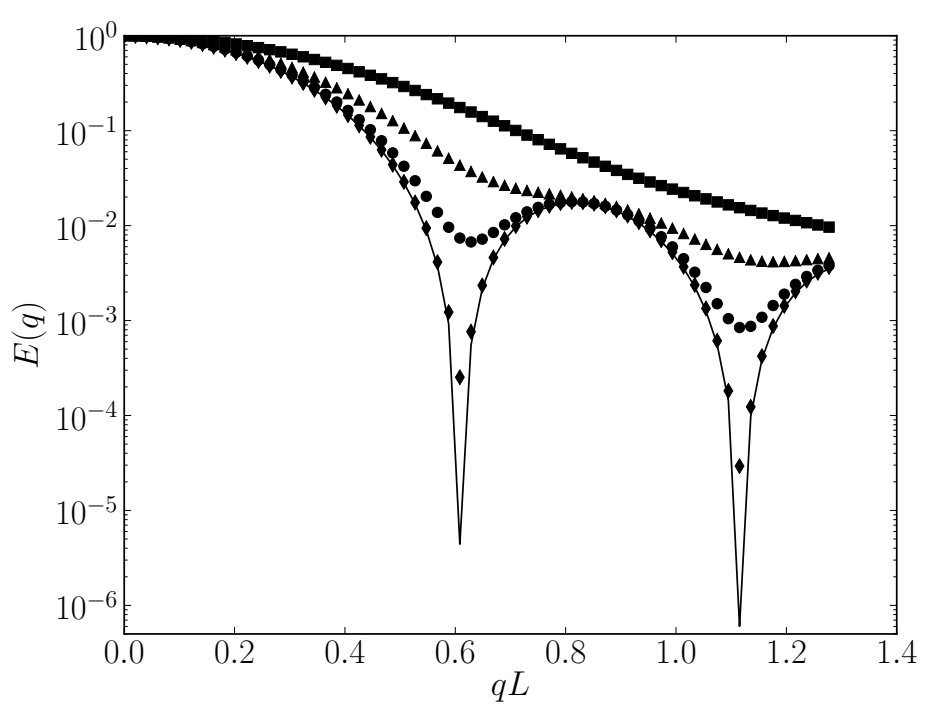

Figure 2.11: PGSE NMR signal amplitude $E(q)$ for $D \Delta / L^{2}=0.2$ (squares), $D \Delta / L^{2}=0.5$ (triangles), $D \Delta / L^{2}=1.0$ (circles) and $D \Delta / L^{2}=2.0$ (diamonds) of a cylindrical pore and the absolute square of the form factor $\left|S_{0}\right|^{2}$ (solid line).

collection of pores consider a matrix of $N$ isolated pores as shown in fig. 2.12 (Callaghan (2011)). Let the center of the pore $i$ be at position $\boldsymbol{r}_{0 i}$ and the local pore density be denoted $\rho_{0 i}$. One may define a local pore coordinate as $\boldsymbol{r}_{\mathrm{p}}=\boldsymbol{r}-\boldsymbol{r}_{0 i}$. when adapting 


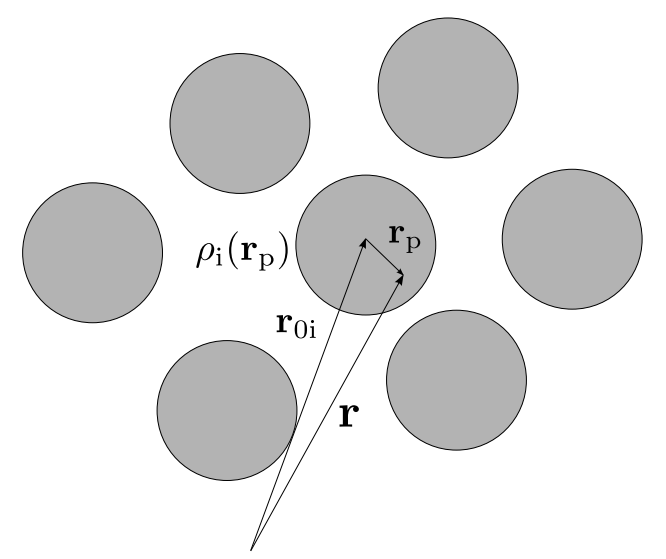

Figure 2.12: Sketch of a matrix of identical cylindrical pores. Shown is the transverse crosssection of the pore system.

eq. 2.41 for the case of $N$ pores one obtains for the spin echo signal (Callaghan (2011))

$$
\begin{aligned}
E_{\infty}(\boldsymbol{q}) & =\frac{1}{N} \sum_{i=1}^{N} \int_{V} \rho_{0 i}\left(\boldsymbol{r}-\boldsymbol{r}_{0 i}\right) e^{-\mathrm{i} 2 \pi \boldsymbol{q} \cdot \boldsymbol{r}} \mathrm{d} \boldsymbol{r} \int_{V} \rho_{0 i}\left(\boldsymbol{r}^{\prime}-\boldsymbol{r}_{0 i}\right) e^{\mathrm{i} 2 \pi \boldsymbol{q} \cdot \boldsymbol{r}^{\prime}} \mathrm{d} \boldsymbol{r}^{\prime} \\
& =\frac{1}{N} \sum_{i=1}^{N} \int_{V} \rho_{0 i}\left(\boldsymbol{r}_{\mathrm{p}}\right) e^{-\mathrm{i} 2 \pi \boldsymbol{q} \cdot\left(\boldsymbol{r}_{0 i}+\boldsymbol{r}_{\mathrm{p}}\right)} \mathrm{d} \boldsymbol{r}_{\mathrm{p}} \int_{V} \rho_{0 i}\left(\boldsymbol{r}_{\mathrm{p}}^{\prime}\right) e^{\mathrm{i} 2 \pi \boldsymbol{q} \cdot\left(\boldsymbol{r}_{0 i}+\boldsymbol{r}_{\mathrm{p}}^{\prime}\right)} \mathrm{d} \boldsymbol{r}_{\mathrm{p}}^{\prime} \\
& =\frac{1}{N} \sum_{i=1}^{N} \int_{V} \rho_{0 i}\left(\boldsymbol{r}_{\mathrm{p}}\right) e^{-\mathrm{i} 2 \pi \boldsymbol{q} \cdot\left(\boldsymbol{r}_{\mathrm{p}}\right)} \mathrm{d} \boldsymbol{r}_{\mathrm{p}} \int_{V} \rho_{0 i}\left(\boldsymbol{r}_{\mathrm{p}}^{\prime}\right) e^{\mathrm{i} 2 \pi \boldsymbol{q} \cdot\left(\boldsymbol{r}_{\mathrm{p}}^{\prime}\right)} \mathrm{d} \boldsymbol{r}_{\mathrm{p}}^{\prime} \\
& =\frac{1}{N} \sum_{i=1}^{N} S_{0 i}(\boldsymbol{q}) S_{0 i}^{*}(\boldsymbol{q}) \\
& =\frac{\left|S_{0}(\boldsymbol{q})\right|^{2}}{}
\end{aligned}
$$

Thus, the signal $E(\boldsymbol{q})$ will be the average squared form factor of the pore system $E_{\infty}(\boldsymbol{q})=\overline{\left|S_{0}(\boldsymbol{q})\right|^{2}}$ and its Fourier transform yields the average pore density autocorrelation function $\overline{\rho^{*}(\boldsymbol{R})}$ (compare fig. 1.3)

$$
\overline{\rho^{*}(\boldsymbol{R})}=\mathcal{F}\left\{\overline{\left|S_{0}(\boldsymbol{q})\right|^{2}}\right\}=\overline{\int_{V} \rho_{0}\left(\boldsymbol{r}_{\mathrm{p}}\right) \rho_{0}\left(\boldsymbol{r}_{\mathrm{p}}+\boldsymbol{R}\right) \mathrm{d} \boldsymbol{r}_{\mathrm{p}}}
$$

This function does not directly return the average pore shape, but contains enough information to extract characteristic length scales of the pores (Callaghan (2011)).

The advantage of diffusive-diffraction PGSE NMR is that it averages the signal of the 
whole sample to yield the spin-echo amplitude. MRI also uses the signal of the whole sample, but it is distributed into voxels of the final image. Thus, diffusive-diffraction PGSE NMR overcomes the signal-to-noise limitation of MRI. However, the spatial distribution of the pore sizes is lost and is replaced by the averaged convolution of all the pore structures present in the sample.

\subsubsection{Magnetic Resonance Pore Imaging (MRPI)}

Magnetic Resonance Pore Imaging was first proposed by Laun et al. (2011) who showed that by a simple modification of the diffusive-diffraction PGSE NMR experiment one may obtain the full complex form factor $S_{0}(\boldsymbol{q})$ of closed pores. This approach promised to answer a long standing question whether it is possible to obtain the pore shape directly by NMR diffusion experiments as posed by Grebenkov (2007) and Shemesh et al. (2012) for example.

In the MRPI experiment the initial narrow gradient pulse in the diffusive-diffractive PGSE NMR experiment (fig. 2.13 (a)) is replaced by a long gradient pulse of duration $\delta_{\mathrm{L}}$ with reduced amplitude $\boldsymbol{G}_{\mathrm{L}}$ as shown in fig. 2.13 (b). The second gradient pulse is intense with amplitude $\boldsymbol{G}_{\mathrm{N}}$ and narrow with duration $\delta_{\mathrm{N}}$, while the spin-echo condition $\boldsymbol{G}_{\mathrm{L}} \delta_{\mathrm{L}}=\boldsymbol{G}_{\mathrm{N}} \delta_{\mathrm{N}}$ has to be full-filled for each gradient step. One has to solve for the ensemble average of the acquired phase as discussed for diffusive diffraction PGSE NMR and given by eq. 2.35. Assuming the short gradient pulse is applied instantaneously $\delta_{\mathrm{N}} \rightarrow 0$ such that $\delta_{\mathrm{L}} \rightarrow T$ one obtains (Laun et al. (2011))

$$
\Phi_{T}=2 \pi \boldsymbol{q} \cdot\left[\frac{1}{T} \int_{0}^{T} \boldsymbol{r}(t) \mathrm{d} t-\boldsymbol{r}(T)\right]
$$

where $\boldsymbol{r}(T)$ is the position of the spin at the time of the narrow gradient $T$. Furthermore, any time integral over the position of a random walker can be replaced by the center of mass of its trajectory (Mitra and Halperin (1995)). The crucial point of the MRPI experiment is that in the limit of infinitely long times $T \rightarrow \infty\left(D T \gg L^{2}\right)$ each diffusing molecule will have been at every point inside the pore with equal probability. In this long time limit, the center of mass of each trajectory converges to the center of mass of 


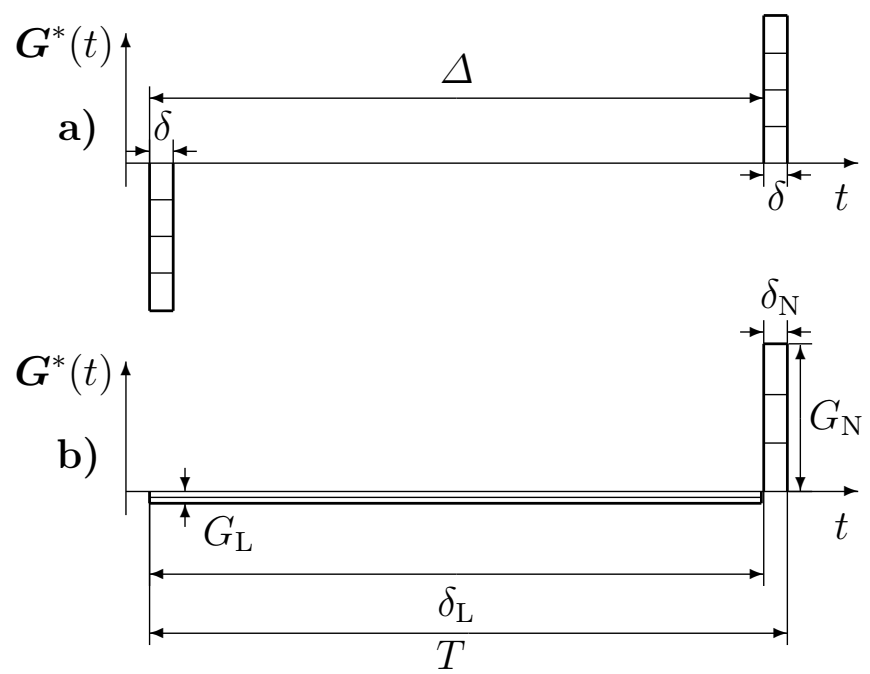

Figure 2.13: Effective gradient schemes of diffusive-diffraction PGSE NMR with two narrow gradients of duration $\delta$ and with "observation time" $\Delta$ (a). MRPI where one of the narrow gradients is extended in duration to $\delta_{\mathrm{L}}$ with reduced amplitude $\boldsymbol{G}_{\mathrm{L}}$, which is balanced by a narrow and intense gradient pulse of duration $\delta_{\mathrm{N}}$ (Laun et al. (2011)). The time $T$ is defined as the total duration of the gradient pulse scheme (b).

the pore space $\boldsymbol{r}_{\mathrm{cm}}$ (Laun et al. (2011))

$$
\lim _{T \rightarrow \infty} \frac{1}{T} \int_{0}^{T} \boldsymbol{r}(t) \mathrm{d} t=\boldsymbol{r}_{\mathrm{cm}} .
$$

Figure 2.14 shows schematically the mean position of the trajectories $\overline{\boldsymbol{r}}_{1,2}$ of two exemplary molecules labeled 1,2. The molecules start at two distinct positions inside the pore space as shown in fig.2.14 (a). As the molecules diffuse in the pore space they are reflected at the pore walls (fig.2.14 (b)) and one may define the mean position of their trajectories as $\overline{\boldsymbol{r}_{\mathrm{i}}}=1 / t \int_{t} \boldsymbol{r}_{\mathrm{i}} \mathrm{d} t^{\prime}$. After the time $T$ and when the condition $D T \gg L^{2}$ is met, the mean positions converge to the center of mass position $\boldsymbol{r}_{\mathrm{cm}}$ of the pore (fig.2.14 (c)). Equation 2.49 and eq. 2.50 can be inserted into eq. 2.35 leaving the ensemble average to be evaluated. For sufficiently long times $T$ the final position $\boldsymbol{r}(T)$ will be uncorrelated to the prior particle positions and one can separate the evaluation of the ensemble average

$$
E_{\infty}(\boldsymbol{q})=\left\langle\exp \left\{\mathrm{i} 2 \pi \boldsymbol{q} \cdot \boldsymbol{r}_{\mathrm{cm}}\right\}\right\rangle\langle\exp \{-\mathrm{i} 2 \pi \boldsymbol{q} \cdot \boldsymbol{r}(T)\}\rangle
$$



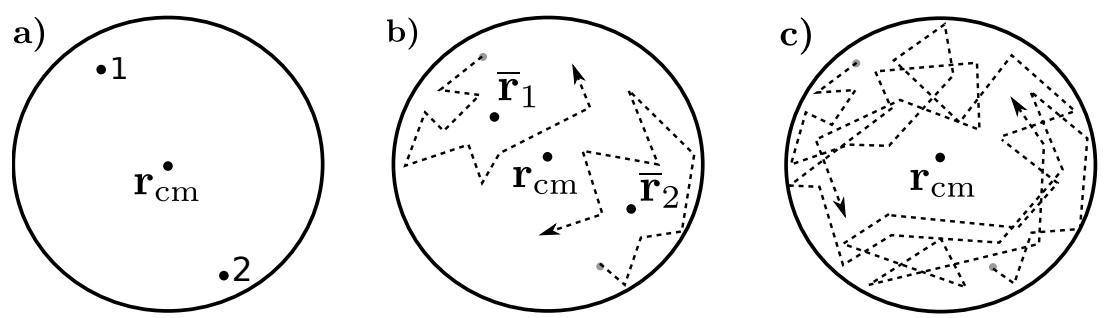

Figure 2.14: The molecules 1,2 start at two distinct positions inside the pore space (a). As the molecules diffuse in the pore space they are reflected at the pore walls and one may define the mean position $\overline{\boldsymbol{r}_{1,2}}$ (b). After the time $T$ and when the condition $D T \gg L^{2}$ is met, the mean positions converge to the center of mass position $\boldsymbol{r}_{\mathrm{cm}}$ of the pore (c)).

The left term yields a complex number and the right term returns the form factor of the pore space

$$
E_{\infty}(\boldsymbol{q})=\frac{1}{V} \exp \left\{\mathrm{i} 2 \pi \boldsymbol{q} \cdot \boldsymbol{r}_{\mathrm{cm}}\right\} S_{0}(\boldsymbol{q})
$$

The complex prefactor in eq. 2.52 will be the same for all pores of the same shape and can therefore be neglected. The corresponding expression for the pore system can be obtained by averaging over all pores following the same concept as for diffusive-diffraction PGSE NMR in the previous section. Thus, the spin echo signal is given by

$$
E_{\infty}(\boldsymbol{q})=\overline{S_{0}(\boldsymbol{q})}
$$

By stepping the gradient amplitudes one acquires the average form factor in the direction of the applied gradients. Thus, this experiment is akin to MRI phase imaging, but with a resolution no more limited by signal-to-noise as it is for MRI.

Note that the required convergence time $T$ may be longer for MRPI as compared to diffusive-diffraction PGSE NMR due to the stronger requirement of convergence to the center of mass of the pore space $\boldsymbol{r}_{\mathrm{cm}}$ as compared to the loss of correlation of the starting $\boldsymbol{r}_{0}(0)$ and final position $\boldsymbol{r}_{1}(\Delta)$. Therefore, depending on the self-diffusion coefficient of the pore fluid $D$ and the characteristic pore length $L$, the required duration of the long gradient pulse may require several hundreds of milliseconds. 


\subsubsection{MRPI resolution}

Signal-to-noise is not the limiting factor for resolution in MRPI, since the signal of the average pore image is arising from the whole sample. This assumption holds for samples which exhibit sufficient NMR signal to be imaged using MRI, but no further subdivision of the voxels in the MRI image is possible to resolve the underlying micro-structure. Another limiting factor for the achievable resolution of MRI and MRPI is the achievable gradient strength by the gradient hardware. The narrow gradient in MRPI acts similar to MRI phase encoding gradients and one may apply similar concepts to derive the resolution of the technique. To make sure that $\boldsymbol{q}$-space is not under sampled, phase increments due to increments of $\boldsymbol{q}$-vectors need to be below $2 \pi$ over the field of view $(F O V)$. Thus, the phase increment $\Delta \Phi$ has to obey the inequality

$$
\Delta \Phi \leq 2 \pi
$$

The increment in phase spread over the $F O V$ using a phase encoding gradient pulse is given by

$$
\Delta \Phi=\gamma \delta \Delta G F O V
$$

where $\Delta G$ is the increment of the gradient strength as shown in fig. 2.15. Inserting

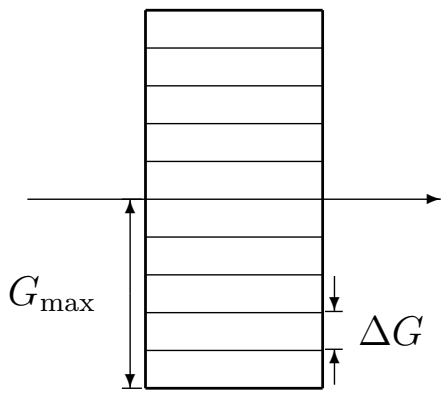

Figure 2.15: Scheme of gradient strength increment $\Delta G$ and the maximum gradient strength $G_{\max }$. 
eq. 2.55 into eq. 2.54 and re-arranging for the field of view yields

$$
F O V=\frac{2 \pi}{\gamma \Delta G \delta}=\frac{1}{\Delta q}
$$

Using bipolar gradient pulses as shown in fig. 2.15 the increment in gradient strength is given by

$$
\Delta G=\frac{2 G_{\max }}{N_{\mathrm{G}}}
$$

where $N_{\mathrm{G}}$ is the number of gradient steps. Inserting eq. 2.57 into eq. 2.56 yields

$$
F O V=\frac{N_{\mathrm{G}} \pi}{\gamma G_{\max } \delta}
$$

The number of points in real space is equal to the number of points in $\boldsymbol{q}$-space. Thus, the resolution of the final image is given by the $F O V$ divided by the number of gradient steps

$$
\Delta z=\frac{F O V}{N_{\mathrm{G}}}=\frac{\pi}{\gamma G_{\max } \delta}
$$

Substituting the definition of $q$ into eq. 2.59 one obtains

$$
\Delta z=\frac{1}{2 q_{\max }}
$$

Note that the factor of two is present due to the use of bipolar gradients and the extra information gained by scanning $\boldsymbol{q}$-space symmetrically. According to eq. 2.60, the resolution of MRPI is determined by the highest gradient intensity applicable by the gradient hardware.

Furthermore, Eq. 2.60 is valid for the MRPI pulse sequence in the case of infinitely long $\delta_{\mathrm{L}}$ and infinitesimally short $\delta_{\mathrm{N}}$. Deviations from this limit will lead to artifacts having to be accounted for in the image analysis (Laun et al. (2012)) as will be discussed in the next sections. 


\subsubsection{Blurring}

If the long gradient is not long enough, such that the limit $D \delta_{L} \gg L^{2}$ is violated, the phase spread of the spins in the pore space cannot be approximated as a delta function anymore. Instead the phase distribution function will have a Gaussian shape which will lead to blurring of the final MRPI image. The width of this distribution can be calculated using the Gaussian phase approximation (Neuman (1974), Robertson (1966), Laun et al. (2012)) or it can be simulated by Monte Carlo simulation (Toumelin et al. (2007)) and other matrix based techniques as suggested by Callaghan (1997) and Grebenkov (2007).

Based on the Gaussian phase approximation, one may show that the probability density $p\left(x_{\mathrm{cm}}\right)$ of finding a molecule with a path having a center of mass position at $x_{\mathrm{cm}}$ is given by (Mitra and Halperin (1995))

$$
p\left(x_{\mathrm{cm}}\right)=\left(2 \pi \sigma_{\mathrm{x}}^{2}\right)^{-1 / 2} \exp \left\{-\frac{x_{\mathrm{cm}}^{2}}{2 \sigma_{\mathrm{x}}^{2}}\right\}
$$

The standard deviation is given by

$$
\sigma_{\mathrm{x}}=\sqrt{\left\langle x_{\mathrm{cm}}^{2}\right\rangle}=L^{2} \sqrt{\frac{2 \xi_{-1} I_{f}}{\delta_{\mathrm{L}} D}},
$$

where $\delta_{\mathrm{L}}$ is the duration of the long gradient in the MRPI sequence as shown in fig. 2.13 (b). Further, $\xi_{-1}$ is a geometry dependent factor, which is $1 / 120$ for parallel planes and $7 / 96$ for cylindrical pores (Grebenkov $(2007)$ ) and $I_{\mathrm{f}}$ is given by $I_{\mathrm{f}}=\int \mathrm{d} t f(t)^{2}$, where $f(t)$ is the temporal gradient profile. Evaluation of $p\left(x_{\mathrm{cm}}\right)$ allows us to visualize the distribution of the center of mass positions of Brownian trajectories during application of the long gradient $\delta_{\mathrm{L}}$. Note that the function $p\left(x_{\mathrm{cm}}\right)$ given by eq. 2.61 corresponds to the phase distribution function after the long gradient and allows to estimate the effect of blurring resulting from a finite duration $\delta_{\mathrm{L}}$ on the pore image.

Figure 2.16 (a) shows the function $p\left(x_{\mathrm{cm}}\right)$ for three times $\delta_{\mathrm{L}}=(200,500,1000 \mathrm{~ms})$ for parallel planes with a separation of $L=20 \mu \mathrm{m}$ and for $\mathrm{H}_{2} \mathrm{O}$ with a self-diffusion coefficient of $D=2.0 \times 10^{-9} \mathrm{~m}^{2} \mathrm{~s}^{-1}$ as obtained by evaluating eq. 2.61. The effect on the pore image is shown in fig. 2.16 (b) as would be obtained if the narrow gradient was 
instantaneous $\delta_{\mathrm{N}} \rightarrow 0$. The profiles were calculated by convoluting the function $p\left(x_{\mathrm{cm}}\right)$ with the pore density function $\rho_{0}(x)$ which is constant in between the parallel planes and zero outside. Laun et al. (2012) give an estimate based on Eq. 2.62 for the duration
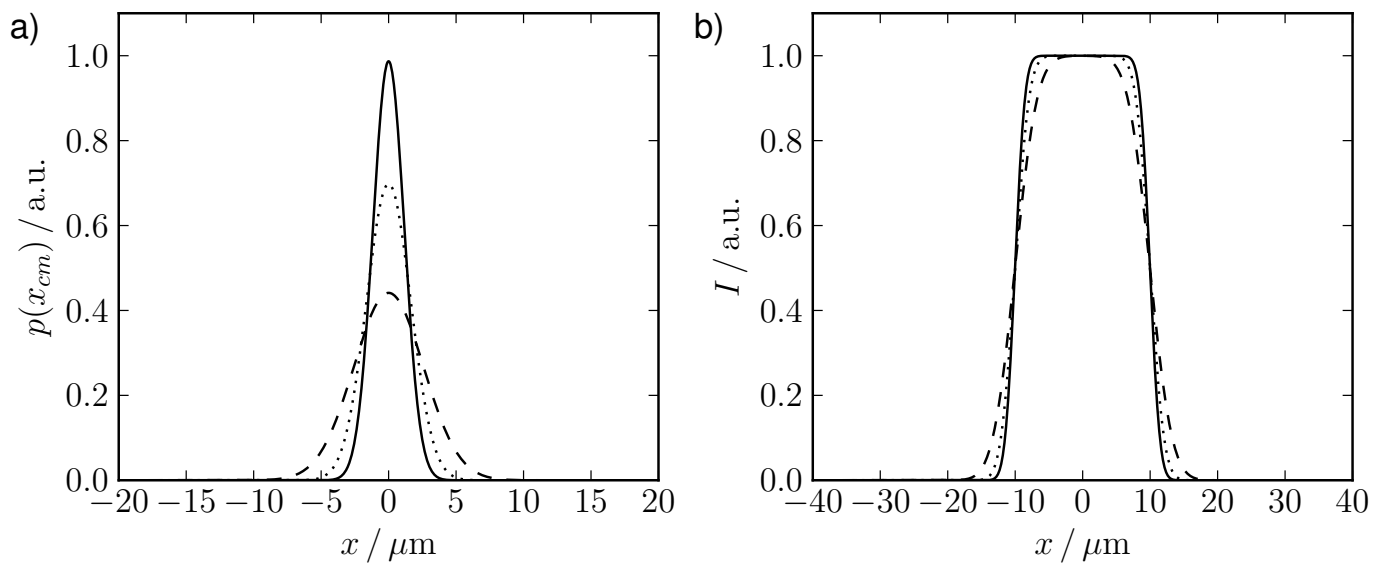

Figure 2.16: Center of mass distribution function $p\left(x_{\mathrm{cm}}\right)$ for gradient duration $\delta_{\mathrm{L}}=10 \mathrm{~ms}$ (dashed), $\delta_{\mathrm{L}}=50 \mathrm{~ms}$ (dotted) and $\delta_{\mathrm{L}}=100 \mathrm{~ms}$ (solid) (a). Expected 1d-MRPI image if the narrow gradient was applied instantaneously $\delta_{\mathrm{N}} \rightarrow 0$ for $\delta_{\mathrm{L}}=200 \mathrm{~ms}$ (dashed), $\delta_{\mathrm{L}}=500 \mathrm{~ms}$ (dotted) and $\delta_{\mathrm{L}}=1000 \mathrm{~ms}$ (solid) (b).

of $\delta_{\mathrm{L}}$ such that the width of this phase spread $\sigma_{\mathrm{x}}$ is smaller than the pixel size $\Delta x$

$$
\delta_{\mathrm{L}} \geq 1000 \Delta x^{2} /(6 D)
$$

\subsubsection{Edge enhancement}

Another image artifact has to be accounted for if the narrow gradient has a finite width $\delta_{\mathrm{N}}$. During that time the molecules may diffuse a distance comparable to the pixel size of the MRPI image. Molecules which are starting near the wall at the beginning of the gradient pulse are likely to be reflected and directed towards the center of the pore. Thus, there will be a region of depleted signal close to the wall. Moreover, due to conservation of the total signal there will be a region of increased intensity shifted towards the center of the pore image. This effect is well known from diffusive-diffraction PGSE NMR (Mitra and Halperin (1995)) and NMR microscopy (Pütz et al. (1991, 1992), Callaghan et al. (1993), De Swiet (1995)) and has been called edge-enhancement. An empirical formula for the shape of $p\left(x_{b}\right)$ has been found by Mitra and Halperin (1995) 
which is well suited to approximate the shift of the signal maximum towards the pore center in case of reflecting pore walls and restricted diffusion.

The effect of a finite refocusing gradient pulse for smooth surfaces and for a locally flat surface is given by Mitra and Halperin (1995)

$$
P\left(x_{\mathrm{b}}\right)=\exp \left\{-\frac{a D \delta_{\mathrm{N}}}{x_{\mathrm{b}}^{2}}\right\}\left[1+b \exp \left\{-c\left(\frac{x_{\mathrm{b}}}{\sqrt{D \delta_{\mathrm{N}}}}+x_{\mathrm{s}, 0}\right)^{2}\right\}\right]
$$

with $a=0.1181, b=7.703, c=0.6361$ and $x_{\mathrm{s}, 0}=1.206$.

Figure 2.17 shows the edge enhancement effect for three times $\delta_{\mathrm{N}}=(1,10,20 \mathrm{~ms})$ on the slab domain. The position of the signal maximum $x_{\max }$ can be obtained from eq. 2.64 by analyzing the maxima of the function and is given by

$$
x_{\max }=0.5926 \sqrt{D \delta_{\mathrm{N}}} .
$$

Note that this position will be shifted further towards the center of the pore in case

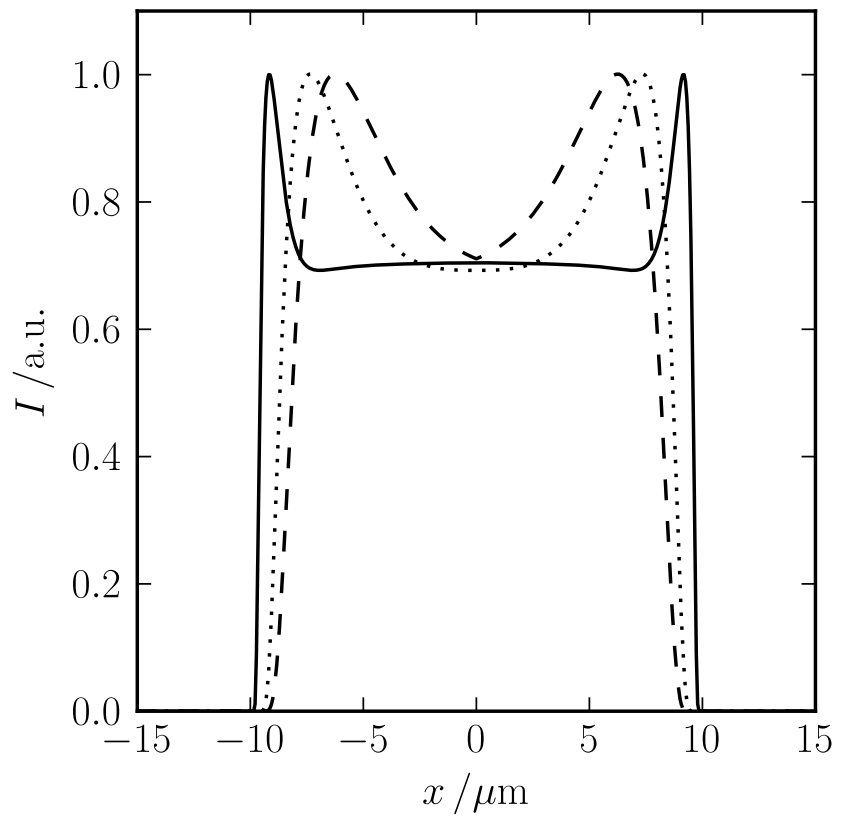

Figure 2.17: Edge enhancement for the parallel plane geometry for the short gradient duration $\delta_{\mathrm{N}}=1 \mathrm{~ms}($ solid$), \delta_{\mathrm{N}}=10 \mathrm{~ms}$ (dotted) and $\delta_{\mathrm{N}}=20 \mathrm{~ms}$ (dashed).

significant blurring due to the finite duration of the long gradient is present. This effect 
may be rationalized when considering that the edge enhanced image has to be convoluted with the Gaussian function (eq. 2.64). Since the edge enhanced image in fig. 2.17 has approximately constant intensity inside the pore and zero outside, the convolution with a Gaussian will shift the peaks further towards the pore center.

\subsection{Inhomogeneous magnetic fields in porous media}

Magnetic fields in porous materials may be inhomogeneous due to magnetic susceptibility differences $\Delta \chi(\boldsymbol{r})$ in different parts of the sample. These susceptibility differences may arise between the solid matrix and the pore fluid as well as due to a distribution of materials and material densities in the solid matrix. NMR experiments are sensitive to the resulting distribution of magnetic field strengths due to the dependence of the Larmor frequency on the magnetic field $\Delta \omega_{0}(\boldsymbol{r})=\gamma \Delta \chi(\boldsymbol{r}) B_{0}$. Especially, for wide distributions of these internal magnetic field strengths as they are common in porous materials it is important to account for their influence on the NMR experiment.

\subsubsection{Coherence pathways of spin- $1 / 2$ nuclei}

This section presents a formalism developed by Hürlimann (2001) for the calculation of the dynamics of the magnetization of spin- $1 / 2$ nuclei in inhomogeneous magnetic fields. This formalism will be used to derive and explain the phase cycling schemes of NMR rf-pulse sequences in sec. 2.3.3 as well as to explain the results of the MRPI PIETA experiments in sec. 4.2.2.2.

Suppose an experiment is conducted in a spatially varying magnetic field $\boldsymbol{B}_{0}(z)$ along the $z$-axis. Let $\Delta \omega_{0}(z)$ be the offset of the Larmor frequency relative to the rf-frequency $\omega_{\text {rf }}$, which represents the frequency of the rf-pulses and the rotating frame of reference. $\omega_{\text {rf }}$ is chosen according to the gyro-magnetic ratio $\gamma$ of the observed nucleus (in this case ${ }^{1} \mathrm{H}$ ) and the polarizing magnetic field $B_{0}$ without the sample. The local offset frequency in the rotating frame of reference is given by

$$
\Delta \omega_{0}(z)=\omega_{\mathrm{rf}}-\gamma\left|\boldsymbol{B}_{0}(z)\right|
$$


Let $B_{1}$ be the strength of the circularly polarized magnetic field of the rf-pulse and let $\varphi$ be its phase. Thus, $\omega_{1}$ is a measure for the strength of the rf-pulse and one may write

$$
\gamma B_{1}(t)=\omega_{1} \cos \left(\omega_{\mathrm{rf}} t+\varphi\right)
$$

During application of an rf-pulse and in the presence of the local offset frequency $\Delta \omega_{0}(z)$ the nutation frequency $\Omega$ of the magnetization is given by $\Omega=\sqrt{\omega_{1}^{2}+\Delta \omega_{0}^{2}}$. Figure 2.18 shows the local effective magnetic field $B_{\text {eff }}$ about which the magnetization is rotating in the rotating frame of reference. The direction of the nutation $\Omega$ is given by the unit vector $\hat{\boldsymbol{n}}$ according to

$$
\hat{\boldsymbol{n}}=\frac{\omega_{1}}{\Omega}[\cos \varphi \hat{\boldsymbol{x}}+\sin \varphi \hat{\boldsymbol{y}}]+\frac{\Delta \omega_{0}}{\Omega} \hat{\boldsymbol{z}}
$$

One may perform a coordinate transformation to the complex circular basis (Hürlimann

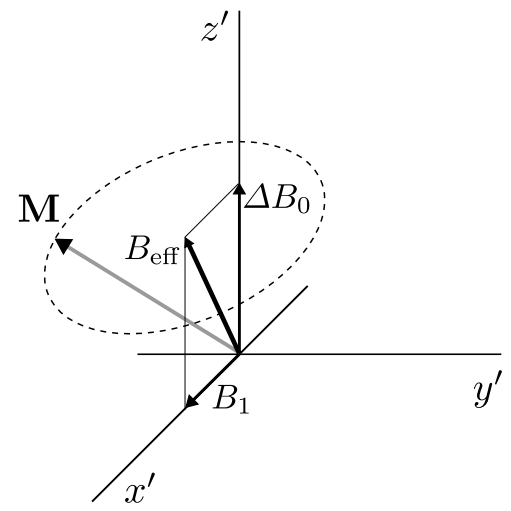

Figure 2.18: Precession of the magnetization vector about the effective magnetic field $B_{\text {eff }}=\Omega / \gamma$ in the rotating frame of reference. Given a local in-homogeneity of the polarizing magnetic field $\Delta B_{0}=\Delta \omega / \gamma$ and the rf-pulse strength $B_{1}$ the direction of $B_{\text {eff }}$ can be found by vector addition of the two magnetic fields.

(2001)) where the magnetization vector is given by the following transformation

$$
\boldsymbol{M}=\left(\begin{array}{c}
M_{+} \\
M_{-} \\
M_{0}
\end{array}\right)=\left(\begin{array}{c}
M_{\mathrm{x}}+\mathrm{i} M_{\mathrm{y}} \\
M_{\mathrm{x}}-\mathrm{i} M_{\mathrm{y}} \\
M_{\mathrm{z}}
\end{array}\right)
$$


This transformation allows to follow the magnetization components according to their coherence, i.e. their acquisition of phase over time. Furthermore, this transformation offers the advantage that the phase evolution of the magnetization components under free precession is diagonalized and is given by the matrix multiplication $\boldsymbol{M}(t)=\boldsymbol{F}(t) \boldsymbol{M}(0)$ which is given component wise by

$$
\left(\begin{array}{c}
M_{+}(t) \\
M_{-}(t) \\
M_{0}(t)
\end{array}\right)=\left(\begin{array}{ccc}
e^{\mathrm{i} \Delta \omega_{0} t} & 0 & 0 \\
0 & e^{-\mathrm{i} \Delta \omega_{0} t} & 0 \\
0 & 0 & 1
\end{array}\right)\left(\begin{array}{c}
M_{+}(0) \\
M_{-}(0) \\
M_{0}(0)
\end{array}\right)
$$

The rotation of the magnetization $\boldsymbol{M}$ during application of an rf-pulse can be described by a $3 \times 3$ matrix $\boldsymbol{\Lambda}(\hat{\boldsymbol{n}}, \theta)$, which can be applied from the left to the magnetization vector

$$
\boldsymbol{M}\left(t_{p}\right)=\boldsymbol{\Lambda}(\hat{\boldsymbol{n}}, \theta) \boldsymbol{M}(t=0)
$$

Applying the rf-pulse for a period $t_{\mathrm{p}}$ will rotate the magnetization vector by an angle $\theta=\Omega t_{p}$ about the direction $\hat{\boldsymbol{n}}$.

The matrix $\boldsymbol{\Lambda}(\hat{\boldsymbol{n}}, \theta)$ mixes the elements of the magnetization vector $M_{+}, M_{-}$and $M_{0}$ due to non-zero off-diagonal elements of $\boldsymbol{\Lambda}(\hat{\boldsymbol{n}}, \theta)$. The elements of $\Lambda$ are labeled by indices $l, m$ with values,+- and 0 . Rewriting eq. 2.71 in matrix notation one obtains

$$
\left(\begin{array}{c}
M_{+}\left(t_{\mathrm{p}}\right) \\
M_{-}\left(t_{\mathrm{p}}\right) \\
M_{0}\left(t_{\mathrm{p}}\right)
\end{array}\right)=\left(\begin{array}{ccc}
\Lambda_{++} & \Lambda_{+-} & \Lambda_{+0} \\
\Lambda_{-+} & \Lambda_{--} & \Lambda_{-0} \\
\Lambda_{0+} & \Lambda_{0-} & \Lambda_{00}
\end{array}\right)\left(\begin{array}{c}
M_{+}(0) \\
M_{-}(0) \\
M_{0}(0)
\end{array}\right)
$$

For example, the matrix element $\Lambda_{0+}$ determines how much magnetization is transferred from the component along the $z$-direction $\left(M_{0}\right)$ into the component $M_{+}$.

The matrix elements $\Lambda_{1, \mathrm{~m}}$ depend on the rf-pulse duration $t_{\mathrm{p}}$, the rf-pulse phase $\varphi$ as well as the local offset frequency $\Delta \omega_{0}(z)$ and the rf-field strength $\omega_{1}$. The indices $l, m$ indicate which elements of the vector $\boldsymbol{M}$ are mixed. Two exemplary elements are given 
by

$$
\begin{aligned}
& \Lambda_{0,+}=\frac{1}{2} \frac{\omega_{1}}{\Omega}\left\{\frac{\Delta \omega_{0}}{\Omega}\left[1-\cos \left(\Omega t_{p}\right)\right]-\mathrm{i} \sin \left(\Omega t_{p}\right)\right\} e^{-\mathrm{i} \varphi} \\
& \Lambda_{+,-}=\frac{1}{2}\left(\frac{\omega_{1}}{\Omega}\right)^{2}\left[1-\cos \left(\Omega t_{p}\right)\right] e^{+\mathrm{i} 2 \varphi} .
\end{aligned}
$$

The expressions for all 9 elements of $\boldsymbol{\Lambda}(\hat{\boldsymbol{n}}, \theta)$ are provided by Hürlimann (2001). The dependence of the matrix elements on the rf-pulse phase according to $\Lambda_{1, \mathrm{~m}} \propto e^{\mathrm{i}(l-m) \varphi}$ can be used to separate different coherence pathways by phase cycling, as will be described in sec 2.3.3.

Consider a pulse sequence of $N$ rf-pulses, which involves the mixing of the magnetization components by each rf-pulse as well as the acquisition of phase according to eq. 2.70 in between the rf-pulses. After each rf-pulse the components $+1,-1,0$ are mixed and one can decompose the final signal into coherence pathways given by the vector $\boldsymbol{p}=\left(p_{1}, p_{2}, \ldots, p_{\mathrm{N}}\right)$, where each element $p_{\mathrm{k}}$ can assume the values $+1,-1$ or 0 . For spin-1/2 nuclei in the absence of any long range couplings the maximum change in coherence $\left|\Delta p_{\mathrm{k}}\right|=|m-l|$ is $\max \left(\left|\Delta p_{\mathrm{k}}\right|\right)=+2$.

For example consider the spin-echo rf-pulse sequence discussed in sec. 2.1.3. The magnetization at time $t=2 \tau$ neglecting the influence of diffusion and relaxation is given by the ordered matrix product

$$
\boldsymbol{M}(2 \tau)=\boldsymbol{F}(\tau) \Lambda\left(180^{\circ}\right) \boldsymbol{F}(\tau) \Lambda\left(90^{\circ}\right) \boldsymbol{M}(0)
$$

where $\boldsymbol{F}(\tau)$ is the free precession matrix and $\Lambda\left(90^{\circ}\right)$ and $\Lambda\left(180^{\circ}\right)$ denote the rf-pulse rotation matrices. One may notice that the free-precession matrix $\boldsymbol{F}(\tau)$ enters twice, one for each time period $\tau$. However, the effect of the free precession matrices on the final phase of the magnetization is opposite to each other caused by the transfer of magnetization from $M_{+}$to $M_{-}$. Selecting the direct pathway $\boldsymbol{p}_{\text {direct }}=(0,+1,-1)$ one may write component wise

$$
M_{0,+1,-1}(2 \tau)=e^{-\mathrm{i} \Delta \omega_{0} \tau} \Lambda_{+-} e^{\mathrm{i} \Delta \omega_{0} \tau} \Lambda_{0+} M_{0}
$$


The effect of free precession cancels at the center of the spin-echo at time $t=2 \tau$.

Hence, the signal contribution of one specific coherence pathway $\boldsymbol{p}$ in case of $N$ rfpulses and for one offset frequency $\Delta \omega_{0}(z)$ is given by

$$
M_{\mathrm{p}_{1}, \mathrm{p}_{2}, \ldots, \mathrm{p}_{\mathrm{N}}}=\left(\prod_{k=1}^{N} \Lambda_{\mathrm{p}_{\mathrm{k}}, \mathrm{p}_{\mathrm{k}-1}}\right) \exp \left\{\mathrm{i} \Delta \omega_{0}(z) \sum_{k=1}^{N} p_{\mathrm{k}} t_{\mathrm{k}}\right\}
$$

The total magnetization $\boldsymbol{M}^{(N)}$ observed after $N$ rf-pulses arising from one position $z$ in the sample is given by the sum over all coherence pathways

$$
\begin{aligned}
\boldsymbol{M}^{(N)}= & \sum_{\mathrm{p}_{1}, \mathrm{p}_{2}, \ldots, \mathrm{p}_{\mathrm{N}-1}}\left[M_{\mathrm{p}_{1}, \mathrm{p}_{2}, \ldots, \mathrm{p}_{\mathrm{N}-1},+1}\left(\frac{\hat{x}-\mathrm{i} \hat{y}}{2}\right)\right. \\
& \left.+M_{\mathrm{p}_{1}, \mathrm{p}_{2}, \ldots, \mathrm{p}_{\mathrm{N}-1},-1}\left(\frac{\hat{x}+\mathrm{i} \hat{y}}{2}\right)+M_{\mathrm{p}_{1}, \mathrm{p}_{2}, \ldots, \mathrm{p}_{\mathrm{N}-1}, 0} \hat{z}\right],
\end{aligned}
$$

which one can rewrite given that $M_{-\mathrm{p}_{1},-\mathrm{p}_{2}, \ldots,-\mathrm{p}_{\mathrm{N}}}=M_{\mathrm{p}_{1}, \mathrm{p}_{2}, \ldots, \mathrm{p}_{\mathrm{N}}}^{*}$ and the final expression is given as

$$
\begin{aligned}
\boldsymbol{M}^{(N)}= & \sum_{p_{1}, p_{2}, \ldots, p_{\mathrm{N}-1}}\left[\operatorname{Re}\left\{M_{p_{1}, p_{2}, \ldots, p_{\mathrm{N}-1},+1}\right\} \hat{x}\right. \\
& \left.+\operatorname{Im}\left\{M_{p_{1}, p_{2}, \ldots, p_{\mathrm{N}-1},+1}\right\} \hat{\boldsymbol{y}}+\operatorname{Re}\left\{M_{p_{1}, p_{2}, \ldots, p_{\mathrm{N}-1}, 0}\right\} \hat{z}\right] .
\end{aligned}
$$

Note that in order to obtain the NMR signal one has to sum the magnetization $\boldsymbol{M}^{(N)}$ for all offset frequencies $\Delta \omega_{0}(z)$.

The number of coherence pathways $\boldsymbol{p}$ grows with the number of rf-pulses with $3^{N}$. However, only a subset of coherence pathways contributes significantly to the magnetization $\boldsymbol{M}^{(N)}$. In strongly in-homogeneous magnetic fields one may find the selection rule

$$
\Delta \omega_{0} \sum_{\mathrm{k}=1}^{N} p_{\mathrm{k}} t_{\mathrm{k}}=0
$$

which can be found considering that at the time of the echo formation the total accumulated phase due to free precession has to be zero. This point will be further explained in sec. 2.3.3.1 on the example of the spin-echo pulse sequence as introduced in sec. 2.1.3. 
The above presented formalism can be utilized to calculate the NMR signal in inhomogeneous fields and to estimate the most significantly contributing coherence pathways.

\subsubsection{Influence of relaxation and self-diffusion on coherence pathways}

As has been shown in sec. 2.1.2 transverse magnetization $M_{+}, M_{-}\left(p_{\mathrm{k}}= \pm 1\right)$ is subject to the transverse relaxation rate $1 / T_{2}$. Similarly, during periods when the magnetization is stored in the $z$-direction $\left(p_{\mathrm{k}}=0\right)$ the magnetization decays with relaxation rate $1 / T_{1}$. These relaxation processes can be accounted for by incorporating eq. 2.9 and eq. 2.11 into eq. 2.77 and accounting for the respective time intervals $t_{\mathrm{k}}$ during which the relaxation processes occur. The expression for each coherence pathway is given by

$$
\exp \left\{-\sum_{k=1}^{N}\left(p_{\mathrm{k}}^{2} / T_{2}+\left(1-p_{\mathrm{k}}^{2}\right) / T_{1}\right) t_{\mathrm{k}}\right\}
$$

Note that the coherence loss with time constant $T_{2}^{*}$ is caused by inhomogeneous magnetic fields and is already accounted for in the free precession matrices. The relaxation rate $1 / T_{2}^{*}$ can be extracted by studying the time dependence of the NMR signal when integrating over all offset frequencies $\Delta \omega_{0}(z)$.

Another influence is the self-diffusion in magnetic field inhomogeneity. A spin bearing molecule undergoes Brownian motion and is gaining a phase $\Phi$ depending on its path in the inhomogeneous magnetic field $\Delta B_{0}(z)=\gamma \Delta \omega_{0}(z)$. This accumulation of phase

during any time interval $t_{\mathrm{k}}$ depends on the coherence of the spin packet $p_{\mathrm{k}}$. According to Hürlimann (2001) the expression for a coherence transfer pathway $\boldsymbol{p}=\left(p_{1}, p_{2} \ldots p_{\mathrm{N}}\right)$ is given by

$$
\begin{aligned}
\left\langle\exp \left\{\mathrm{i} \sum_{k=1}^{N} p_{\mathrm{k}} \phi_{\mathrm{k}}\right\}\right\rangle_{\phi_{1}, \ldots, \phi_{\mathrm{N}}}= & \exp \left\{-\gamma^{2} g^{2} D\left[\sum_{k=1}^{N} \frac{1}{3} p_{\mathrm{k}}^{2} t_{\mathrm{k}}^{3}+\sum_{k=1}^{N} p_{\mathrm{k}} t_{\mathrm{k}} \sum_{l=1}^{k} p_{\mathrm{l}} t_{\mathrm{l}} T_{1}\right.\right. \\
& \left.\left.+\sum_{k=1}^{N} p_{\mathrm{k}} t_{\mathrm{k}}\left(t_{\mathrm{k}}+T_{\mathrm{k}}\right) \sum_{l=k+1}^{N} p_{\mathrm{l}} t_{\mathrm{l}}\right]\right\}
\end{aligned}
$$

where $T_{l}$ is the sum of of all the durations of free precession from the first instance 
a non-zero coherence is created up to the beginning of the $l$-th pulse. The expression for the magnetization $M_{\mathrm{p}_{1}, \mathrm{p}_{2}, \ldots, \mathrm{p}_{\mathrm{N}}}$ given by eq. 2.77 given a specific coherence pathway $\boldsymbol{p}=\left(p_{1}, p_{2} \ldots p_{\mathrm{N}}\right)$ has to be multiplied by the terms for relaxation (eq. 2.82) and selfdiffusion (eq. 2.83) and is given by

$$
\begin{aligned}
M_{\mathrm{p}_{1}, \mathrm{p}_{2}, \ldots, \mathrm{p}_{\mathrm{N}}} & =\left(\prod_{k=1}^{N} \Lambda_{\mathrm{p}_{\mathrm{k}}, \mathrm{p}_{\mathrm{k}-1}}\right) \exp \left\{\mathrm{i} \Delta \omega_{0} \sum_{k=1}^{N} p_{\mathrm{k}} t_{\mathrm{k}}\right\} \\
& \times \exp \left\{-\sum_{k=1}^{N}\left(\frac{p_{\mathrm{k}}^{2}}{T_{2}}+\frac{1-p_{\mathrm{k}}^{2}}{T_{1}}\right) t_{\mathrm{k}}\right\} \\
& \times\left\langle\exp \left\{\mathrm{i} \sum_{k=1}^{N} p_{\mathrm{k}} \phi_{\mathrm{k}}\right\}\right\rangle_{\phi_{1}, \ldots, \phi_{\mathrm{N}}} .
\end{aligned}
$$

The magnetization $\boldsymbol{M}^{(N)}$ can be found by summing over all contributing coherence pathways $\boldsymbol{p}$ according to eq. 2.79. These terms have been used to study CPMG rf-pulse sequences in inhomogeneous fields and to estimate the influence on the measured $T_{2}$ and $D$ values by Song (2002).

\subsubsection{Phase cycles for the selection of desired coherence pathways}

In this thesis a novel rf-pulse sequence will be introduced in sec. 3.1.1 for which a special phase cycle was constructed. The procedure applied is outlined below. In NMR experiments one is usually interested in one specific coherence pathway $\boldsymbol{p}=\left(p_{1}, p_{2} \ldots p_{\mathrm{N}}\right)$ or a specific subset of all possible pathways. Therefore, one has to separate this set of pathways from all other pathways that contribute to the signal and fulfill the selection rule (eq. 2.81). One possibility is to utilize the dependence of the rf-pulse matrices $\Lambda$ on the rf-pulse phase $\varphi$ as shown in eq. 2.74. Bodenhausen et al. (1984) and Bain (1984) introduced a procedure to construct a phase cycling scheme which is now widely applied. This procedure allows the selection of a specific coherence pathway by changing the rfpulse phase scheme from scan to scan, i.e. by repeating the NMR experiment with a changed rf-pulse phase scheme several times and adding the resulting signals. Let $\Delta p_{\mathrm{i}}=p_{\mathrm{i}}-p_{\mathrm{i}-1}$ denote the coherence transfer difference induced by the $i$-th rf-pulse, then the rf-pulse phase enters the expression eq. 2.84 for the magnetization due to the 
dependence of the elements of $\Lambda$ on the rf-pulse phase as

$$
\exp \left\{-\mathrm{i} \Delta p_{\mathrm{i}} \varphi\right\}
$$

For the transitions of the ${ }^{1} \mathrm{H}$ spins in spin-echo experiments one has the following possible values for the coherence transfer difference $\Delta p_{\mathrm{i}} \in\{-2,-1,0,+1,+2\}$. For the construction of the phase cycle, one has to list all possible changes in $\Delta p_{\mathrm{i}}$ for a particular rf-pulse $i$ and mark the desired transfer pathway. For example let the set of possible transitions under the influence of the $i$-th rf-pulse be given by

$$
\Delta p_{\mathrm{i}}:\{-2, \underline{-1},(0),(1), 2\},
$$

where the underlined value is the desired transition and the transitions in brackets shall be eliminated. Let the other transitions be of negligible influence on the final signal such that they can be ignored. If $\Delta p_{\text {i }}$ shall be chosen out of a range of $K$ possibilities the number of steps $N_{\mathrm{i}}$ in the $i$-th rf-pulse phase has to be at least equal to $K$. In the example above there are three considered coherence transfer values $K=3$ and therefore the minimum number of phase steps is $\min \left(N_{\mathrm{i}}\right)=3$. Note that $N_{\mathrm{i}}$ is equal to the number of scans $N_{\text {scan }}$ that have to be utilized, since the rf-pulse phase is only changed from scan to scan.

The corresponding rf-pulse phase $\varphi_{\mathrm{i}}$ has to be set to the following values

$$
\varphi_{\mathrm{i}}=\frac{k_{\mathrm{i}} 2 \pi}{N_{\mathrm{i}}}
$$

with $k_{\mathrm{i}}=0,1, \ldots N_{\mathrm{i}}-1$. The above analysis has to be repeated for each rf-pulse in the sequence. The goal of phase cycling is that the signal for the desired transfer pathway always adds up while the undesired ones are cancelled after all scans are completed. The most common way to achieve this is to shift the rf-receiver reference phase $\varphi_{\text {rec }}$ from scan to scan according to the equation

$$
\varphi_{\mathrm{rec}}=-\sum_{i=1}^{N} \Delta p_{\mathrm{i}} \varphi_{\mathrm{i}}
$$


where $\Delta p_{\mathrm{i}}$ is the desired coherence transfer difference of the $i$-th rf-pulse and $\varphi_{\mathrm{i}}$ is its phase for one particular scan. This way the desired coherence transfer pathway will always be recorded with the same phase in the receiver, while the undesired pathways will cycle in phase and average to zero upon addition.

\subsubsection{Phase cycle of the spin-echo sequence}

As an example for the application of the procedure of Bodenhausen et al. (1984) and Bain (1984) consider the spin-echo pulse sequence as discussed in sec. 2.1.3. All possibilities for the coherence pathway vector $\boldsymbol{p}=\left(p_{0}, p_{1}, p_{2}\right)$ are given by combining all possible values $p_{\mathrm{k}} \in\{-1,0,+1\}$ for each time period $t_{\mathrm{k}}$. However, assuming thermal equilibrium at the start of the experiment all pathways start with $z$-magnetization $\left(p_{0}=0\right)$. The possibilities for $\boldsymbol{p}$ are given in the first column of tab. 2.1.

Table 2.1: Possible coherence pathway vectors $\boldsymbol{p}$ of the spin-echo pulse sequence (left). After retaining only the measurable coherences with $p_{2}=-1$ (assuming perfect quadrature detection) there are only three contributing coherence pathways (center). After phase cycling one may filter the direct pathway $\boldsymbol{p}_{\text {direct }}=(0,+1,-1)$.

\begin{tabular}{lll}
$\boldsymbol{p}$ & $\boldsymbol{p}\left(p_{2}=-1\right)$ & $\boldsymbol{p}_{\text {direct }}$ \\
\hline & & \\
$0,+1,+1$ & & \\
$0,+1, \quad 0$ & & \\
$0,+1,-1$ & $0,+1,-1$ & \\
$0, \quad 0,+1$ & & \\
$0, \quad 0, \quad 0$ & & \\
$0, \quad 0,-1$ & $0, \quad 0,-1$ & \\
$0,-1,+1$ & & \\
$0,-1, \quad 0$ & & \\
$0,-1,-1$ & $0,-1,-1$ & \\
\hline
\end{tabular}

Assuming perfect quadrature detection, only the pathways ending in $p_{2}=-1$ contribute to the signal, see tab. 2.1 (center). Further information about quadrature detection and why only the coherence $p_{2}=-1$ can be measured can be found in Callaghan (2011). The aim of phase cycling in this case is to filter for the direct coherence pathway $\boldsymbol{p}_{\text {direct }}=(0,+1,-1)$. Focusing on the $180^{\circ}$ rf-pulse the contributing pathway transitions 
are

$$
\Delta p_{2}:\{\underline{-2},(-1),(0)\}
$$

where the underlined value is the desired transition and the transitions in brackets have to be eliminated. As discussed in the previous section, the phase increment is given by $\Delta \varphi_{2}=k_{2} 2 \pi / 3$, where $k_{2}=0,1,2$. The phase of the signal due to the second rf-pulse and the receiver phase is given by

$$
\exp \left\{-\mathrm{i}\left(\Delta p_{2} \varphi_{2}+\varphi_{\text {rec }}\right\}=\exp \left\{-\mathrm{i}\left(\Delta p_{2} k_{2} 2 \pi / 3-\sum_{i=1}^{N} \Delta p_{\mathrm{i}} \varphi_{\mathrm{i}}\right)\right\}\right.
$$

where $\varphi_{\text {rec }}$ in the right term was replaced by eq. 2.88 .

First, consider the desired pathway $\boldsymbol{p}_{\text {direct }}=(0,+1,-1)$ with $\Delta p_{2}=-2$. For the first scan with $k_{2}=0$ one obtains the phase factor

$$
\exp \{-\mathrm{i} 0\}=1
$$

the second step with $k_{2}=1$ is giving

$$
\exp \{-\mathrm{i}(2 \pi / 3+4 \pi / 3\}=\exp \{-\mathrm{i} 2 \pi\}=1
$$

and the third step with $k_{2}=2$ yields

$$
\exp \{-\mathrm{i}(4 \pi / 3+8 \pi / 3\}=\exp \{-\mathrm{i} 4 \pi\}=1
$$

Upon addition of the signal arising from all three scans one obtains an amplitude equal to three times the value as obtained by a single scan.

Once the receiver phase $\varphi_{\text {rec }}$ has been determined for all scans one may proceed to analyze the phases of the signal of the undesired pathways. Note that the sum in eq. 2.90 is not re-evaluated. For the pathway with $\Delta p_{2}=0$ one obtains for the first scan $\left(k_{2}=0\right)$

$$
\exp \{-\mathrm{i}(0)\}=1
$$


for the second scan $\left(k_{2}=1\right)$

$$
\exp \{-\mathrm{i}(4 \pi / 3)\}
$$

and for the third scan $\left(k_{2}=2\right)$ one obtains

$$
\exp \{-\mathrm{i}(8 \pi / 3)\}
$$

The sum of the last two exponentials yields -1 and thus adding the first scan yields zero. Hence, this pathway is eliminated after addition of the three scans. Similar expressions can be found for the pathway with $\Delta p_{2}=-1$. Thus, the number of scans for the phase cycle of the spin-echo pulse sequence is $N_{\text {scan }}=3$.

Historically, the phases for rf-pulses had to be multiples of $\pi / 2$ and thus the phase cycle was expanded to $N_{\text {scan }}=4$ phase steps, see tab. 2.2. In case of imperfections in the

Table 2.2: Phase cycle of the spin-echo pulse sequence with $N_{\text {scan }}=4$ steps. The rf-pulse phases $\varphi_{1}, \varphi_{2}, \varphi_{\text {rec }}$ correspond to the first and second rf-pulse and the reference phase during acquisition as shown in fig. 2.4.

\begin{tabular}{ccc}
\hline \multicolumn{2}{c}{ Pulse phases } & Acquisition \\
\cline { 1 - 2 }$\varphi_{1}$ & $\varphi_{2}$ & $\varphi_{\text {rec }}$ \\
\cline { 1 - 2 } 0 & 0 & $3 \pi / 2$ \\
0 & $\pi / 2$ & $\pi / 2$ \\
0 & $\pi$ & $3 \pi / 2$ \\
0 & $3 \pi / 2$ & $\pi / 2$ \\
\hline
\end{tabular}

quadrature detection as they might occur in some analog detector systems, the number of phase steps $N_{\text {scan }}=4$ has the advantage of eliminating the additional coherence pathways with $p_{2}=+1$.

\subsubsection{Phase cycle of the CPMG sequence}

Figure 2.19 shows the CPMG pulse sequence including the coherence levels of spin$1 / 2$ nuclei. The brackets indicate the section of the rf-pulse sequence that is repeated multiple times to create a train of $180^{\circ}$ rf-pulses. The number of coherence pathways 


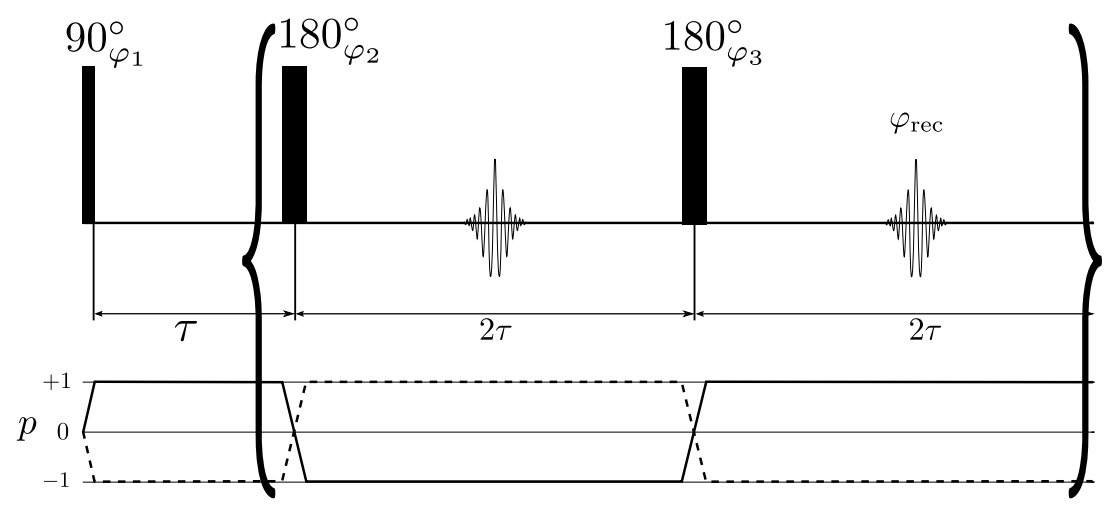

Figure 2.19: Carr-Purcell-Meiboom-Gill (CPMG) rf-pulse sequence. The leading $90^{\circ}$ rf-pulse is creating coherence of the spin system (transverse magnetization $M_{+}$and $M_{-}$). A train of $180^{\circ}$ rf-pulses follows each separated by twice the initial $90^{\circ}-180^{\circ}$ rf-pulse separation $(2 \tau)$. The curly brackets enclose the segment that is repeated $n / 2$ times to yield a CPMG rf-pulse sequence with $n$ spin echos. The three levels of coherence $p_{\mathrm{k}} \in\{-1,0+1\}$ are shown below. The direct pathway $\boldsymbol{p}_{\text {direct }}=(-1,+1,-1,+1, \ldots)$ and its conjugate $\tilde{\boldsymbol{p}}_{\text {direct }}=(+1,-1,+1,-1, \ldots)$ are indicated by a solid and a dotted line, respectively.

grows with $3^{n}$, where $n$ is the number of rf-pulses and the number of spin-echos. Typical CPMG experiments involve such a large number of rf-pulses $n$ that the construction of a complete phase cycle becomes impracticable. In practice, a reduced phase cycle is commonly applied, which accounts for the most important imperfections in CPMG experiments. This phase cycle consists of $N_{\text {scan }}=2$ steps, which are listed in tab. 2.3. In practice one often employs a 4-step phase cycle, which also accounts for imperfect

Table 2.3: Phase cycle of the CPMG pulse sequence with $N_{\text {scan }}=2$ steps (Meiboom and Gill (1958)). The rf-pulse phases $\varphi_{1}, \varphi_{2}, \varphi_{3}, \varphi_{\text {rec }}$ correspond to the rf-pulses and the reference phase during acquisition as shown in fig. 2.19. Shifting the phase of the $90^{\circ}$ rf-pulse $\varphi_{1}$ and the reference phase $\varphi_{\text {rec }}$ corrects for quadrature detection errors and eliminates magnetization that is excited by the $180^{\circ} \mathrm{rf}$-pulses.

\begin{tabular}{cccc}
\hline \multicolumn{3}{c}{ Pulse phases } & Acquisition \\
\cline { 1 - 2 }$\varphi_{1}$ & $\varphi_{2}$ & $\varphi_{3}$ & $\varphi_{\mathrm{rec}}$ \\
\hline & & & \\
0 & $\pi / 2$ & $\pi / 2$ & $\pi / 2$ \\
$\pi$ & $3 \pi / 2$ & $3 \pi / 2$ & $3 \pi / 2$ \\
\hline
\end{tabular}

quadrature detection. However, even the 4-step phase cycle might not be enough to filter the most destructive coherence pathways and more elaborate phase schemes such as discussed in the next section may have to used. 


\subsubsection{Phase Incremented Echo Train Acquisition (PIETA)}

Baltisberger et al. (2012) introduced a new phase cycling scheme that allows to unambiguously extract desired coherence pathways from Echo Train Acquisition (ETA) experiments. ETA is a general expression for a train of spin echos due to a train of rfpulses, which can be appended to an NMR experiment or may represent a stand-alone experiment like CPMG. This approach is called Phase Incremented Echo Train Acquisition (PIETA), because the phase of every other refocusing pulse $\varphi_{\mathrm{P}}$ is incremented as a single variable from scan to scan. PIETA applied to the CPMG pulse sequence is shown in fig. 2.20. In conventional CPMG experiments one acquires the signal in the dimen-

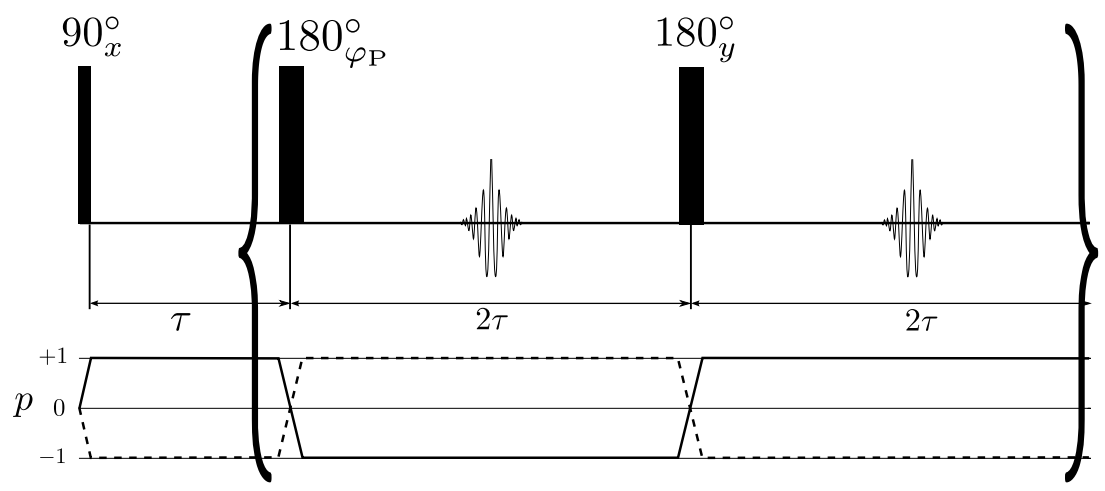

Figure 2.20: PIETA phase scheme as applied to the CPMG pulse sequence, where every other $180^{\circ} \mathrm{rf}$-pulse is phase incremented in $\varphi_{\mathrm{P}}$. The phase of the $90^{\circ} \mathrm{rf}$-pulse and every even $180^{\circ}$ rf-pulse stays fixed.

sions of echo count $n$ and the time $t$ after the last refocusing pulse, which yields the signal $E(n, t)$. PIETA adds another dimension to CPMG experiments in the direction of the phase increments $\varphi_{\mathrm{P}}$ and thus the signal is given by the matrix $E\left(n, t, \varphi_{\mathrm{P}}\right)$. The signal can be reduced back to two dimensions by applying a Fourier transform of the signal with respect to the phase $\varphi_{\mathrm{P}}$

$$
E(n, t, \Delta P)=\int E\left(n, t, \varphi_{\mathrm{P}}\right) \exp \left\{-\mathrm{i} \Delta P \varphi_{\mathrm{P}}\right\} \mathrm{d} \varphi_{\mathrm{P}}
$$

which can be seen as a Fourier transform with respect to the phase $\varphi_{\mathrm{P}}$ with $\Delta P$ being its conjugate space. This Fourier transform is followed by extracting cross-sections through the $\Delta P$ and echo count $n$ dimension according to the desired pathway equation $\Delta P(n)$. 
Thus, PIETA de-aliases coherence pathways according to their total change of coherence $\Delta P$, which is induced by the rf-pulses which are phase cycled with $\varphi_{\mathrm{P}}$.

The desired pathway in the CPMG experiment is the direct pathway $\boldsymbol{p}_{\text {direct }}=(0,+1,-1, \ldots,+1,-1)$. The corresponding coherence transfer change vector is given by $\Delta \boldsymbol{p}_{\text {direct }}=(+1,-2,+2,-2, \ldots,+2,-2)$. Since the coherence transfer change vector of the direct coherence pathway $\Delta \boldsymbol{p}_{\text {direct }}$ contains the collection of the highest values $( \pm 2)$ for a spin-1/2 system, the cumulative change in coherence $\Delta P$ is highest among all pathways. It is therefore unique for this pathway and it may be extracted without any interference from other pathways.

Consider for example the case of two segments in fig. 2.20 and thus in total four $180^{\circ}$ rf-pulses and $n=4$ spin-echos. Following eq. 2.85 , the first $180^{\circ}$ rf-pulse adds a phase factor of

$$
\exp \left\{-\mathrm{i} \Delta p_{2} \varphi_{\mathrm{P}}\right\}
$$

where $\Delta p_{2}$ is the second entry in the coherence transfer vector. Thus, for the direct pathway this value is $\Delta p_{2}=-2$. The second $180^{\circ}$ rf-pulse adds a phase factor that is constant from scan to scan and can thus be neglected in this analysis. The third $180^{\circ}$ rf-pulse however adds another phase factor of $\varphi_{4}=-\varphi_{\mathrm{P}} \Delta p_{4}$. For the direct pathway the coherence transfer of this pulse is again $\Delta p_{4}=-2$. Thus defining $\Delta P=-\left(\Delta p_{1}+\Delta p_{4}\right)$, where the - sign was introduced, since the second and fourth $180^{\circ}$ rf-pulse inverted the total acquired phase up to their occurrence. The total phase at the time of the $n=4$ spin-echo is then given by

$$
\varphi_{\text {tot }}=\Delta P \varphi_{\mathrm{P}}=4 \varphi_{\mathrm{P}}
$$

Since eq. 2.97 can be seen as a Fourier transform, the signal is decomposed in its "frequency" components $\Delta P$ and the direct pathway will yield a signal at $\Delta P(n=4)=4$.

Note that if one measures an odd number of spin-echos e.g. $n=3$, the analysis changes slightly. Since one always measures coherences ending in $M_{-1}$ the direct pathway is given by $\boldsymbol{p}_{\text {direct }}=(0,-1,+1, \ldots,+1,-1)$ and the coherence transfer vector is given 
by $\Delta \boldsymbol{p}_{\text {direct }}=(-1,+2,-2, \ldots,+2,-2)$. Thus the total change in coherence is given by $\Delta P=\Delta p_{2}+\Delta p_{4}$ since the total phase has not yet been inverted by the fourth rf-pulse. Thus, the total phase acquired for the third $(n=3)$ spin-echo is given by

$$
\varphi_{\text {tot }}=\Delta P \varphi_{\mathrm{P}}=-4 \varphi_{\mathrm{P}}
$$

and thus this spin-echo yields a signal at the "frequency" component $\Delta P(n=3)=-4$.

One may generalize the above consideration to $n$ spin echos, which leads to the desired pathway equation for the case of the direct coherence pathway given by

$$
\Delta P(n)= \begin{cases}2 M & \text { for even } n \\ -2 M & \text { for odd } n\end{cases}
$$

where $M$ is the number of segments with pairs of $180^{\circ}$ rf-pulses. Figure 2.21 (left) shows the signal at the center of the spin-echo $E(n, t=\tau, \Delta P)$ after Fourier transformation for well adjusted rf-pulse durations. The direct pathway is represented by the two diagonals with values $\Delta \boldsymbol{P}=(-2,2,-4,4,-6,6 \ldots)$. Since the rf-pulses are well adjusted, all other pathways receive negligible intensity. Figure 2.21 (right) shows the same experiment repeated with the duration of the $180^{\circ}$ rf-pulses deliberately misset to the duration of $90^{\circ}$ rf-pulses. The undesired coherence pathways with $|\Delta P|<\left|\Delta P_{\text {direct }}\right|$ receive much more intensity. However, it is still possible to extract just the signal corresponding to the direct pathway along the diagonals although with a much smaller signal amplitude. The number of phase increments has to be chosen such that the Nyquist-Shannon criterion (Shannon (1949)) is fulfilled. In case of PIETA the sample frequency $1 / \Delta \varphi_{\mathrm{P}}$ is determined by $\Delta \varphi_{\mathrm{P}}=2 \pi / N_{\text {scan }}$ and has to be at least double the frequency which shall be detected, which is given by $\Delta P(n)$.

In case of the CPMG PIETA experiment the highest frequency is given by $|\Delta P(n)|=2 M$ and thus $N_{\text {scan }}=4 M$. Since each segment is comprised of two echoes the number of phase increments in dependence on the echo count is given by

$$
N_{\text {scan }}=2 n
$$



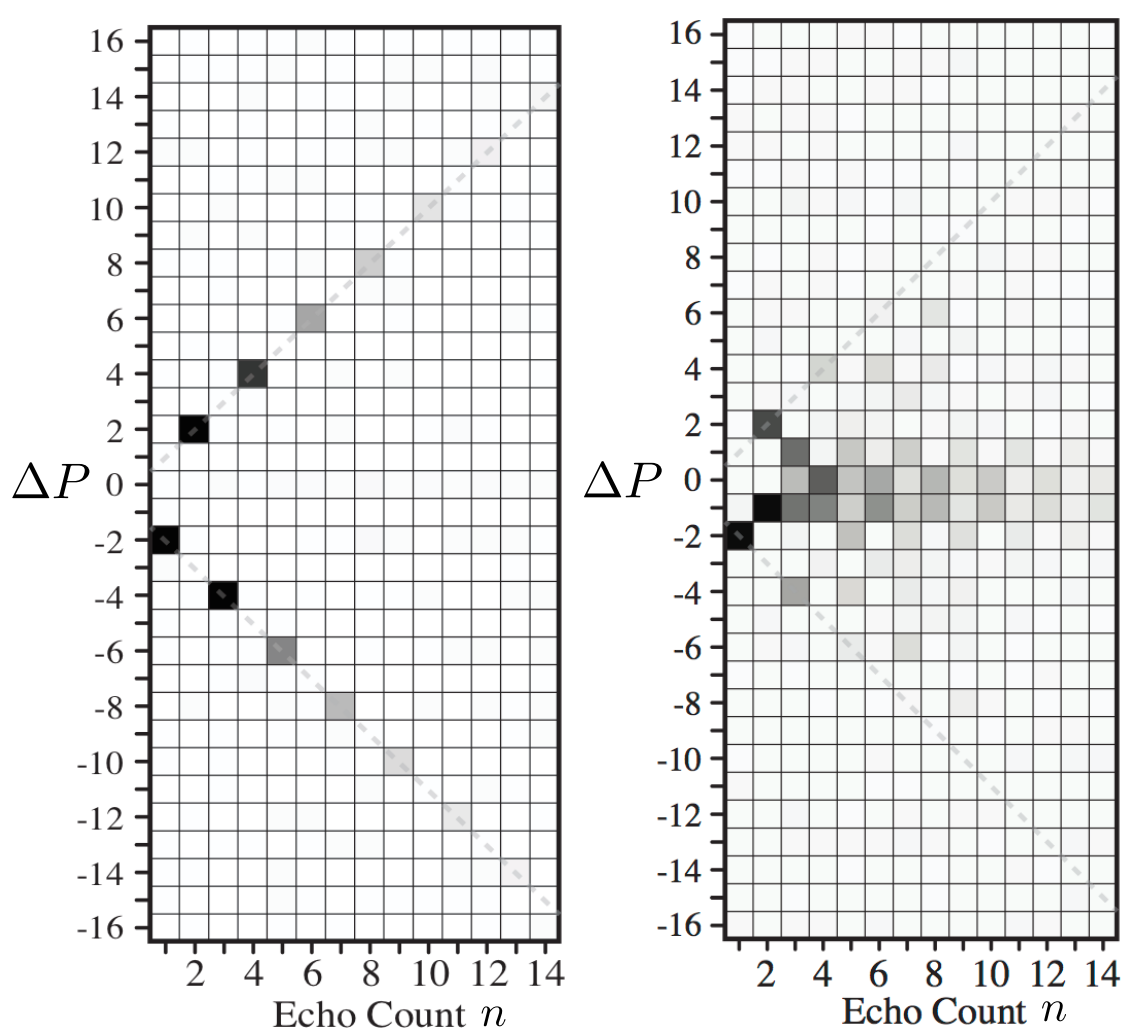

Figure 2.21: Cumulative coherence transfer $\Delta P$ versus spin-echo count $n$. The signal at the spin-echo center $E(n, t=\tau, \Delta P)$ is shown for a well adjusted $180^{\circ}$ rf-pulse duration (left). In the second experiment the rf-pulse duration was misset to the duration of $90^{\circ}$ rf-pulses (right). Reprinted from Baltisberger et al. (2012).

Thus, PIETA may increase the experimental time when many spin-echos are measured, however also the signal-to-noise is improved and it provides additional information. In this thesis PIETA is applied to recover distorted spin-echo amplitudes for CPMG based experiments and it will be utilized to gain a deeper understanding of CPMG NMR relaxation measurements in general.

\subsection{Multiple Correlation Function technique}

The Multiple Correlation Function (MCF) technique was specially developed to solve PGSE NMR problems in closed pore systems. In this thesis it was chosen to simulate the MRPI signal amplitude due to the accuracy and speed of computation of MCF as well as the possibility to study arbitrary temporal gradient patterns with relative 
ease. Grebenkov (2007) introduced this matrix based simulation technique with which he reproduced many results from the PGSE NMR diffusion literature. The derivation of the MCF technique given here is mainly based on the review by Grebenkov (2007), an educational contribution written by Grebenkov (2008) and the recent review by Laun et al. (2012).

The macroscopic equation governing the complex valued magnetization density $m(\boldsymbol{r}, t)=m_{x}(\boldsymbol{r}, t)+$ i $m_{y}(\boldsymbol{r}, t)$ was first introduced by Bloch (1946) and then modified by Torrey (1956) to include the effect of diffusion. This equation is known as the BlochTorrey equation and is given by

$$
\frac{\partial}{\partial t} m(\boldsymbol{r}, t)=D \Delta m(\boldsymbol{r}, t)-\mathrm{i} \gamma B(\boldsymbol{r}, t) m(\boldsymbol{r}, t)
$$

The first term describes the evolution due to diffusion with diffusion coefficient $D$. The second term describes the magnetic field encoding due to the magnetic field $B(\boldsymbol{r}, t)$, which can be expressed as the superposition given by

$$
B(\boldsymbol{r}, t)=B_{0}+f(t) \beta \tilde{G}(\boldsymbol{r})
$$

Note that this expression is similar to eq. 2.17, but has to be modified to separate the time dependence $f(t)$ of the gradient from its maximum intensity $\beta$ and its spatial dependence $\tilde{G}(\boldsymbol{r})$. The static polarizing magnetic field $B_{0}$ leads to a common phase factor for all magnetization densities $m(\boldsymbol{r}, t)$ in the sample and can thus be dropped in the analysis. In the dimensionless function $f(t)$ the application of a $180^{\circ}$ rf-pulse is taken into account by inverting the function $f(t)$ for $t<t_{180^{\circ}}$. The re-phasing condition (eq. 2.16) is re-formulated to

$$
\int_{0}^{T} \mathrm{~d} t f(t)=0 .
$$

Both functions $f(t)$ and $\tilde{G}(\boldsymbol{r})$ are dimensionless and are normalized to one and $f(t)$ is piece wise smooth on $[0, T]$, while $\tilde{G}(\boldsymbol{r})$ is a smooth function on the domain of the pore 
space $\Omega_{\mathrm{p}}$. Given these re-definitions the Bloch-Torrey equation is given by

$$
\left(\frac{\partial}{\partial t}-D \Delta+\mathrm{i} \gamma \beta f(t) \tilde{G}(\boldsymbol{r})\right) m(\boldsymbol{r}, t)=0
$$

\subsubsection{Neumann boundary condition}

As has been discussed in sec. 1.2 the diffusing molecules may experience different degrees of permeability at the pore walls. The Neumann boundary condition describes the case of no surface relaxation or zero permeability through the boundary of the confining domain $\Omega_{\mathrm{p}}$ and is given by

$$
\frac{\partial}{\partial \boldsymbol{n}} m(\boldsymbol{r}, t)=0 \quad \forall \boldsymbol{r} \in \partial \Omega_{\mathrm{p}}
$$

where $\partial / \partial \boldsymbol{n}$ denotes the derivative perpendicular to the boundary $\partial \Omega_{\mathrm{p}}$. One ansatz for solving eq. 2.106 is to expand the magnetization density $m(\boldsymbol{r}, t)$ in the basis of eigenfunctions $u_{\mathrm{m}}(\boldsymbol{r})$ of the Laplace operator

$$
m(\boldsymbol{r}, t)=\sum_{\mathrm{m}}^{\infty} c_{\mathrm{m}}(t) u_{\mathrm{m}}(\boldsymbol{r})
$$

with integer index $m=0,1,2, \ldots$ Note that eq. 2.108 is similar to the expansion of the propagator in eigenfunctions of the confining domain given by eq. 1.8. The Neumann boundary condition has to be applied to the eigenfunctions $u_{\mathrm{m}}(\boldsymbol{r})$ and eigenvalues $\lambda_{\mathrm{m}}$ following the relations

$$
\begin{aligned}
& \Delta u_{\mathrm{m}}(\boldsymbol{r})+\frac{\lambda_{\mathrm{m}}}{L^{2}} u_{\mathrm{m}}(\boldsymbol{r})=0 \quad\left(\boldsymbol{r} \in \Omega_{\mathrm{p}}\right), \\
& \frac{\partial}{\partial n} u_{\mathrm{m}}(\boldsymbol{r})=0 \quad\left(\boldsymbol{r} \in \partial \Omega_{\mathrm{p}}\right) .
\end{aligned}
$$

To find the coefficients $c_{\mathrm{m}}(t)$ one substitutes eq. 2.108 into the Bloch Torrey equation eq. 2.103, multiplies with the complex conjugate of the eigenfunctions $u_{\mathrm{m}}^{*}(\boldsymbol{r})$ and inte- 
grates over $\Omega_{\mathrm{p}}$. The resulting set of ordinary differential equations is given by

$$
\frac{\mathrm{d}}{\mathrm{d} t} c_{\mathrm{m}}(t)+\frac{D \lambda_{\mathrm{m}}}{L^{2}} c_{\mathrm{m}}(t)+\mathrm{i} \gamma \beta L \sum_{\mathrm{m}^{\prime}=0}^{\infty} \mathcal{B}_{\mathrm{m}, \mathrm{m}^{\prime}} c_{\mathrm{m}^{\prime}}(t)=0,
$$

where $L$ is the characteristic dimension of the confining domain e.g. the radius of a cylinder and $\mathcal{B}$ is an infinite dimensional matrix given by

$$
\mathcal{B}_{\mathrm{m}, \mathrm{m}^{\prime}}=\int_{\Omega_{\mathrm{p}}} \mathrm{d} \boldsymbol{r} u_{\mathrm{m}}^{*}(\boldsymbol{r}) \tilde{G}(\boldsymbol{r}) u_{\mathrm{m}^{\prime}}(\boldsymbol{r})
$$

The set of differential equations given by eq. 2.111 can be rewritten by seeing the coefficients $c_{\mathrm{m}}(t)$ as components of an infinite dimensional vector $C(t)$. The set of differential equations in matrix representation can be written as

$$
T \frac{\mathrm{d}}{\mathrm{d} t} C(t)=-\left(p \Lambda+\mathrm{i} q_{\mathrm{G}} \mathcal{B}\right) C(t)
$$

with the solution

$$
C(t)=\exp \left\{-\left(p \Lambda+\mathrm{i} q_{\mathrm{G}} \mathcal{B}\right) t / T\right\} C(0)
$$

Here, the diagonal matrix of dimensionless eigenvalues was defined as

$$
\Lambda_{\mathrm{m}, \mathrm{m}^{\prime}}=\delta_{\mathrm{m}, \mathrm{m}^{\prime}} \lambda_{\mathrm{m}}
$$

Two new dimensionless quantities were introduced, the reduced self-diffusion coefficient

$$
p=\frac{D T}{L^{2}}
$$

and the generalized gradient intensity

$$
q_{\mathrm{G}}=\gamma \beta T L
$$


We followed the notation used by Laun et al. (2012) and denote the generalized gradient intensity as $q_{\mathrm{G}}$ to discern it from the gradient wave vector $q=(2 \pi)^{-1} \gamma G \delta$.

The NMR signal $E$ can be obtained by integrating $m(\boldsymbol{r}, t)$ eq. 2.108 over the domain $\Omega_{\mathrm{p}}$. Assuming that the detection coil has a homogeneous detection profile one arrives at

$$
\begin{aligned}
E & =\int_{\Omega_{\mathrm{p}}} m(\boldsymbol{r}, t) \mathrm{d} \boldsymbol{r}=\sum_{\mathrm{m}=0}^{\infty} c_{\mathrm{m}}(t) \int_{\Omega_{\mathrm{p}}} u_{\mathrm{m}}(\boldsymbol{r}) \mathrm{d} \boldsymbol{r} \\
& =V \sum_{\mathrm{m}=0}^{\infty} c_{\mathrm{m}}(t) c_{\mathrm{m}}(0)
\end{aligned}
$$

Substituting the solution eq. 2.114 already found for the vector $\boldsymbol{C}(t)$ one arrives at

$$
\begin{aligned}
E & =V \boldsymbol{C}^{*}(0) \exp \left\{-\left(p \Lambda+\mathrm{i} q_{\mathrm{G}} \mathcal{B}\right) t / T\right\} \boldsymbol{C}(0) \\
& =\boldsymbol{U}^{*} \exp \left\{-\left(p \Lambda+\mathrm{i} q_{\mathrm{G}} \mathcal{B}\right) t / T\right\} \boldsymbol{U}
\end{aligned}
$$

where a new vector $\boldsymbol{U}=V^{1 / 2} \boldsymbol{C}(0)$ has been introduced. At $t=0$ the magnetization density is uniform which is equivalent to assuming that there is no relaxation prior to the onset of the gradient and thus one has

$$
m(\boldsymbol{r}, t=0)=\frac{1}{V}
$$

where $V$ is the volume of the confining domain $\Omega_{\mathrm{p}}$. The initial condition given by eq. 2.122 implies that

$$
c_{\mathrm{m}}(0)=V^{-1 / 2} \delta_{\mathrm{m}, 0}
$$

Therefore, $\boldsymbol{C}(0)$ has only one entry in case of the Neumann boundary condition and the expression eq. 2.120 is equivalent to taking the first diagonal element according to

$$
E=\left[\exp \left\{-\left(p \Lambda+\mathrm{i} q_{\mathrm{G}} \mathcal{B}\right) t / T\right\}\right]_{0,0}
$$

In case the temporal profile deviates from $f(t)=1$, one can subdivide the interval $[0, T]$ into a number of sub-intervals $K$ of duration $\tau=T / K . f(t)$ is equal to $f(k \tau)$ 
on the $k^{\text {th }}$ sub-interval by approximating the temporal profile as piece wise constant on these sub-intervals. The signal is then found by evaluating the ordered matrix product

$$
E \approx\left[\prod_{k=1}^{K} \exp \left\{-\left(p \Lambda+\mathrm{i} q_{\mathrm{G}} f(k \tau) \mathcal{B}\right) \tau / T\right\}\right]_{0,0}
$$

One has to compute the matrices $\mathcal{B}$ and $\Lambda$ for the chosen domain $\Omega_{\mathrm{p}}$ and spatial profile $\tilde{G}(\boldsymbol{r})$ only once. Then the time dependence $f(t)$ (the usual experimental variation) of the magnetic field can be approximated by eq. 2.125 and its influence on the NMR signal E can be studied (Grebenkov (2008)). The MCF approach will be especially useful for the MRPI pulse sequence discussed in sec. 3.1.1 since it contains many intervals with gradient pulses. Note that the matrix $\mathcal{B}$ has been computed for constant gradients for the slab, cylinder and sphere domain and can be found in Grebenkov (2007). The matrices $\mathcal{B}$ for various triangular domains are given by Laun et al. (2012). Furthermore, one may find implementations into Matlabßand Python code on "http://www.nitrc.org/projects/mcftool". A general overview over geometrical properties of Laplacian eigenfunctions is given by Grebenkov and Nguyen (2013).

\subsubsection{Fourier boundary condition}

In the case of a finite surface relaxation $\rho$ the boundary condition eq. 2.107 has to be rewritten as

$$
\frac{\partial}{\partial n} u_{\mathrm{m}}(\boldsymbol{r})+\frac{h}{L} u_{\mathrm{m}}(\boldsymbol{r})=0 \text { on } \partial \Omega_{\mathrm{p}}
$$

where the dimensionless surface relaxivity

$$
h=L \rho / D
$$

has been defined. In order to account for the effect of surface relaxation the signal attenuation has to be normalized with the signal without gradient

$$
E=\frac{\mathbf{U}^{*} \exp \left\{-\left(p \Lambda+\mathrm{i} q_{\mathrm{G}} \mathcal{B}\right) t / T\right\} \mathbf{U}}{\mathbf{U}^{*} \exp \{-p \Lambda t / T\} \mathbf{U}}
$$


However, the drawback of this solution is that the eigenfunctions and eigenvalues have to be re-evaluated for each value of $h$.

An alternative approach was suggested by Grebenkov (2008) which introduced an extra term in the Bloch-Torrey equation. The term $\kappa \tilde{\mathbf{B}}(\boldsymbol{r})$ is proportional to a generalized relaxation rate $\kappa=p h$ and a spatial distribution of relaxation centers $\tilde{\mathbf{B}}(\boldsymbol{r})$. In this case the exponential evaluated for the signal eq. 2.124 contains an extra term proportional to $\kappa \tilde{\mathcal{B}}$ with

$$
\tilde{\mathcal{B}}_{\mathrm{m}, \mathrm{m}^{\prime}}=\int_{\Omega_{\mathrm{p}}} \mathrm{d} \boldsymbol{r} u_{\mathrm{m}}^{*}(\boldsymbol{r}) \tilde{\mathbf{B}}(\boldsymbol{r}) u_{\mathrm{m}}{ }^{\prime}(\boldsymbol{r})
$$

Since this expression is similar to eq. 2.112 one may proceed to implement surface relaxation $\tilde{\boldsymbol{B}}(\boldsymbol{r})$ using similar expressions as for a spatial dependence of the magnetic field $\tilde{\boldsymbol{G}}(\boldsymbol{r})$. The case of uniform surface relaxation is implemented by letting the spatial distribution $\tilde{\mathbf{B}}(\boldsymbol{r})$ be localized near the boundary $\partial \Omega_{\mathrm{p}}$. In case of a vanishingly small thickness of $\tilde{\mathbf{B}}(\boldsymbol{r})$ on $\partial \Omega_{\mathrm{p}}$ the volume integral in eq. 2.129 reduces to the boundary integral

$$
\tilde{\mathcal{B}}_{\mathrm{m}, \mathrm{m}^{\prime}}^{s}=\int_{\partial \Omega_{\mathrm{p}}} \mathrm{d} \boldsymbol{r} u_{\mathrm{m}}^{*}(\boldsymbol{r}) \tilde{\mathbf{B}}(\boldsymbol{r}) u_{\mathrm{m}},(\boldsymbol{r})
$$

The signal for arbitrary temporal gradient profiles $f(t)$ is then approximated as

$$
E \approx\left[\prod_{k=1}^{K} \exp \left\{-\left(p \Lambda+\mathrm{i} q_{\mathrm{G}} f(k \tau) \mathcal{B}+p h \tilde{\mathcal{B}}^{s}\right) \tau / T\right\}\right]_{0,0}
$$

This approach has the advantage that one has to compute the boundary integral eq. 2.130 only once and one can scale the surface relaxivity through the parameter $h$. The Fourier boundary may be useful for studying semi-permeable pores such as biological cells as well as porous structures with significant surface relaxation such as porous rocks in oilfields. 



\section{Experimental}

\subsection{MRPI pulse sequence design}

As discussed in sec. 2.2.3, in order to achieve the limiting condition $D \delta_{\mathrm{L}} \gg L^{2}$ the duration of the long gradient $\delta_{\mathrm{L}}$ may need to be on the order of several $100 \mathrm{~ms}$ or more. During this time $\delta_{\mathrm{L}}$ the magnetization has to reside in the transverse plane and is therefore subject to particular experimental conditions and limitations. Thus, the MRPI pulse sequence as shown in fig. 2.13 (b) may have to be adapted to utilize and extend the available time most efficiently. Furthermore, MRPI utilizes two gradient pulses with different durations $\left(\delta_{\mathrm{L}}\right.$ and $\left.\delta_{\mathrm{N}}\right)$ as well as amplitudes $\left(G_{\mathrm{L}}\right.$ and $\left.G_{\mathrm{N}}\right)$, which have to be matched to fulfill the echo condition eq. 2.16. This requirement poses additional challenges for the application of the gradient pulses as will be discussed in detail in sec. 3.2.2 and sec. 3.2.3.

The limitations as imposed by the instruments and the sample can be categorized as follows

- Internal gradients: Self-diffusion of spin bearing molecules in an internal gradient leads to an additional coherence loss and thus increases the effective transverse relaxation rate $1 / T_{2}^{\mathrm{eff}}$ (Robertson $\left.(1966)\right)$.

- Gradient non-linearities and offsets: The set gradient strength by the controlling software may not be equal to the actual gradient strength delivered by the system. One of the causes can be the heating of the sensing resistor in the current controlled amplifier. Furthermore, any voltage offset may render bipolar gradients non symmetric. Both effects can lead to mismatches and a distorted echo formation as well as additional spin echo attenuation (Price (1998)). 
- Eddy currents: The rapidly changing magnetic fields due to the switching of gradient pulses may induce eddy currents in the surfaces of conducting materials surrounding the gradient coils. Their effect scales with the slew rate $\mathrm{d} I / \mathrm{dt}$ of the gradient pulses and the final gradient strength. Eddy currents, in turn, generate magnetic fields affecting the nuclear magnetization, which may appear as gradient mismatches and may lead to additional spin echo attenuation (Price (1998)).

- Concomitant fields: According to Maxwells equations any magnetic field gradient has to be accompanied by an additional gradient field perpendicular to the applied gradient field (see sec. 2.1.5). If uncompensated concomitant fields may also lead to echo distortions and additional spin echo attenuation (Norris and Hutchison (1990), Bernstein et al. (1998)).

- Lab main power: One main source of noise is the residual hum of the direct current (DC) power supply of the gradient amplifier, which is generated by rectifying the alternating current (AC) of the lab main power (Galvosas et al. (2001)). In this thesis this source of noise was converted into a reproducible mismatch by triggering the pulse sequence to the phase of the lab main $\mathrm{AC}$ power source as described in Galvosas et al. (2001). The resulting mismatch was subsequently corrected with the help of read gradients.

One may note that in diffusive-diffractive PGSE NMR some of the above stated effects may have none or a much reduced effect due to the symmetry in the gradient pulse pattern. According to Norris and Hutchison (1990) and Bernstein et al. (1998) concomitant fields will be equally strong if two identical, but opposite gradient pulses are applied and thus their effect is compensated. However, in MRPI one has to apply two gradients of very different durations and amplitudes and thus novel approaches to pulse sequence design were applied.

\subsubsection{1d-MRPI}

In this work, one key innovation to reduce the influence of experimental artifacts is to employ a CPMG like pulse sequence (discussed in sec. 2.1.4). Karlicek and Lowe 
(1980) showed that CPMG based PGSE NMR experiments may minimize the influence of internal gradients on the transverse relaxation rate $1 / T_{2}^{\text {eff }}$. As an illustrative example, fig. 3.1 (a) shows a pulsed gradient (black) in the presence of an internal gradient, which distorts the amplitude of the applied gradient (grey). In fig. 3.1 (b) the pulsed gradient

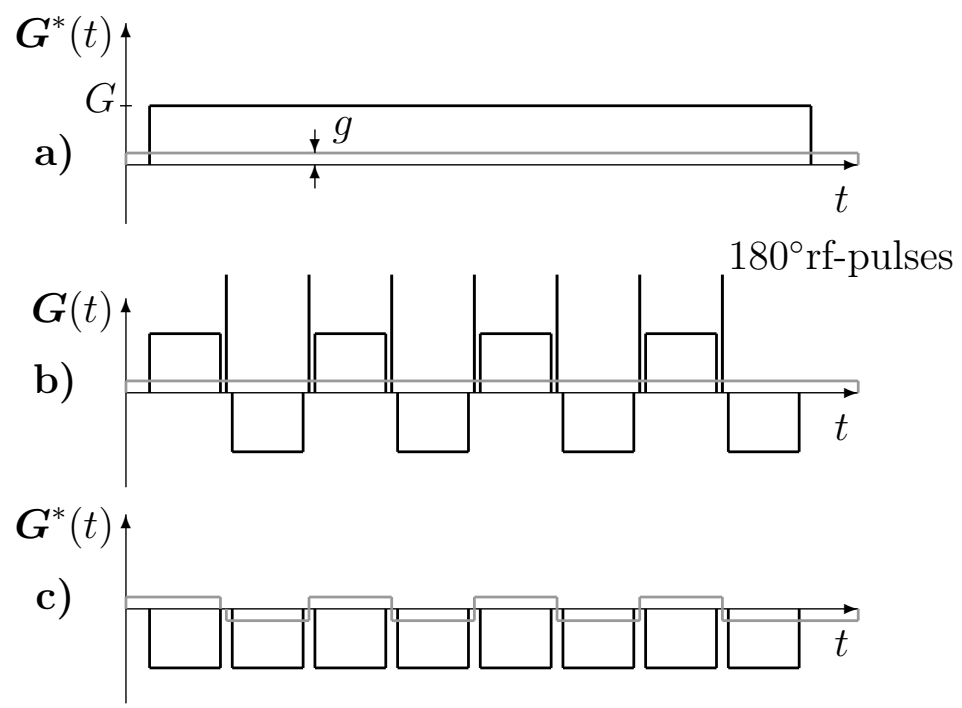

Figure 3.1: Internal gradient compensation utilizing a CPMG like rf-pulse sequence. Pulsed gradient with amplitude $G$ (black) in the presence of an internal gradient, which distorts the amplitude of the applied gradient with amplitude $g$ (grey) (a). The pulsed gradient is interspersed by $180^{\circ} \mathrm{rf}$-pulses and is purposefully applied with alternating polarity in the laboratory reference frame (black). The internal gradient retains its direction in the laboratory reference frame (grey) (b). Effective pulsed gradient $G^{*}(t)$ (black) and the effective internal gradient (grey) (c). Adapted from Laun et al. (2012).

is interspersed by $180^{\circ} \mathrm{rf}$-pulses, which allows the application of the pulsed gradient in both directions in the laboratory reference frame (black). In contrast, the internal gradient retains its direction in the laboratory reference frame (grey). Figure 3.1 (c) shows the effective pulsed gradient (black) and the effective internal gradient (grey). The cumulative effect of the internal gradient is compensated with each second gradient pulse. Additionally, it is vital to reduce the rf-pulse separation to reduce the effective transverse relaxation rate $1 / T_{2}^{\text {eff }}$ given by eq. 2.13 . The second term in eq. 2.13 describes the influence of the self-diffusion of spin bearing molecules in the internal gradient $\boldsymbol{g}$, which can be minimized by the variation of $\tau$ and its extrapolation to zero (Karlicek and Lowe (1980), Kärger et al. (1988)). In doing so, one may utilize a longer time for 
the application of a long gradient, which is crucial for the MRPI technique to fulfill the condition $D \delta_{\mathrm{L}} \gg L^{2}$.

Figure 3.2 (a) shows the CPMG like rf-pulse sequence as utilized in this work. The rf-pulse phases $\varphi_{\mathrm{i}}$ are adapted for the special requirements of the MRPI approach as will be discussed in sec. 3.1.1.1. In order to apply the CPMG concept to MRPI the long

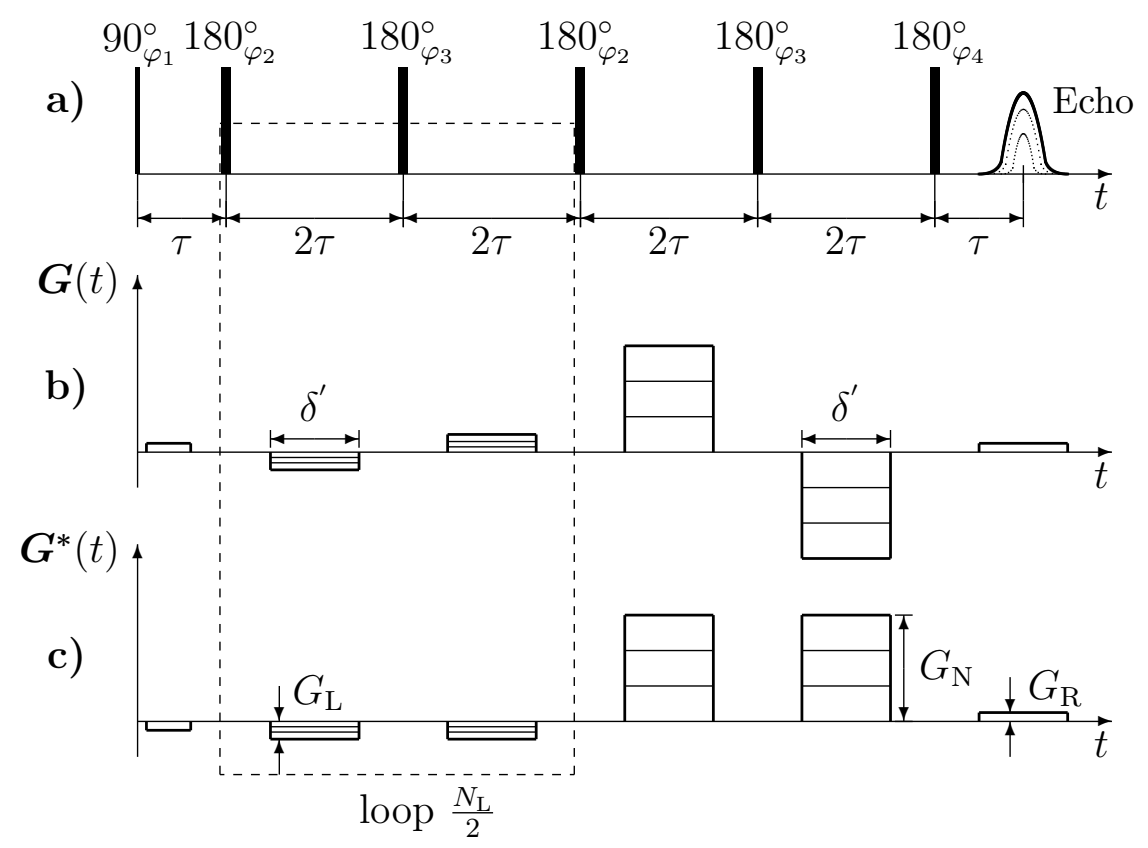

Figure 3.2: 1d-MRPI pulse sequence with a CPMG like rf-pulse scheme (a). The long gradient is replaced by $N_{\mathrm{L}}$ gradient pieces with gradient amplitude $G_{\mathrm{L}}$ and the narrow gradient was split into two intense gradient pulses with gradient amplitude $G_{\mathrm{N}}$. The gradients are shown relative to each other as applied in the laboratory reference frame $(\boldsymbol{G}(t))$ (b). The effective gradient pulse scheme $\boldsymbol{G}^{*}(t)$ shows that the gradient pulses $G_{\mathrm{L}}$ add up and are balanced by the two gradient pulses $G_{\mathrm{N}}$ (c). In practice the gradient pulses are ramped in order to reduce eddy currents (not shown).

gradient $\boldsymbol{G}_{\mathrm{L}}$ was split into a succession of short gradient pulses of duration $\delta^{\prime}$, which were applied centered between the $180^{\circ}$ rf-pulses (fig. 3.2 (b)). Similarly, the narrow gradient pulse $G_{\mathrm{N}}$ was split into two intense gradient pulses each of duration $\delta^{\prime}$. It is important to note that in the laboratory reference frame the gradients were applied in alternating polarity, such that their effect on the spin system is cumulative. The effective gradient pulse scheme $\boldsymbol{G}^{*}(t)$ as seen by the spin system is schematically shown in fig. 3.2 (c). The effective pulsed gradient $\boldsymbol{G}^{*}(t)$ consists of $N_{\mathrm{L}}$ gradient pieces of strength $\boldsymbol{G}_{\mathrm{L}}$ which are balanced by two narrow gradients with gradient strength $\boldsymbol{G}_{\mathrm{N}}$. In this work we employed 
two narrow gradients as well as an even number $N_{\mathrm{L}}$ of long gradient pieces to ensure that internal gradients and offsets are canceled with each second gradient pulse. Additionally, all gradients were ramped to reduce eddy currents and to ensure a controlled gradient pulse shape (not shown in fig. 3.2).

Furthermore, we introduced read gradients $\boldsymbol{G}_{\mathrm{R}}$ in the interval between the leading $90^{\circ}$ and the first $180^{\circ}$ rf-pulse and during acquisition, see fig. 3.2 (b) and (c). The acquisition of the spin echo in the presence of the read gradient allows to monitor the echo position in the time domain (Callaghan (1990)). This approach is vital to detect gradient mismatches. Suppose for example that one narrow gradient is applied with amplitude difference $\Delta G_{\mathrm{N}}$. The magnetization will thus acquire a phase difference $\Delta \Phi=\gamma \Delta G_{\mathrm{N}} \delta^{\prime}$. In the presence of the read gradient $G_{\mathrm{R}}$ this additional phase $\Delta \Phi$ will be rephased at a different time than expected for the spin echo without the gradient mismatch (Galvosas et al. (2001), Hrovat and Wade (1980)). This time difference $\Delta t$ is given by the condition that the phase difference $\Delta \Phi$ is compensated thus giving

$$
\gamma G_{\mathrm{R}} \Delta t=\gamma \Delta G_{\mathrm{N}} \delta^{\prime} \Rightarrow \Delta t=\frac{\Delta G_{\mathrm{N}} \delta^{\prime}}{G_{\mathrm{R}}}
$$

Furthermore, the read gradient allows the separation of the wanted signal (the direct coherence pathway $\boldsymbol{p}=(0,+1,-1,+1,-1, \ldots,+1,-1))$ from unwanted coherence pathways as will be discussed in the next section.

\subsubsection{Elimination of unwanted coherence pathways}

The introduction of many rf-pulses can give rise to unwanted spin echoes due to additional coherence pathways (Hürlimann (2001)), especially if the rf-pulses are applied in inhomogeneous fields as they are common in porous materials (see sec. 2.3.1). Unwanted coherence pathways can either be filtered using phase cycling as discussed in sec. 2.3.3 or using gradient pulses which was first proposed by Bax et al. (1980).

Filtering using gradient pulses is based on the selection rule given by eq. 2.81. According to this selection rule, the acquired phase due to inhomogeneities including gradient pulses has to be rephased to allow for a spin-echo to occur. The gradient schemes of 
PGSE NMR experiments are usually designed to dephase and rephase a selected coherence pathway e.g. the direct pathway $\boldsymbol{p}_{\text {direct }}$. In this case, other pathways which miss or invert part of the gradient pulses may be dephased and cannot contribute to the spin-echo anymore.

The MRPI pulse sequence utilizes a distinct gradient pattern which limits the number of contributing coherence pathways if the gradient amplitudes are sufficiently high. For example, take a coherence pathway that misses one of the long gradient pieces of amplitude $\boldsymbol{G}_{\mathrm{L}}$ such as $\boldsymbol{p}=(0,+1,0,+1,-1, \ldots,+1,-1)$, where the magnetization is stored in $z$-direction during the time interval $t_{2}$. The additional phase of this particular coherence pathway will be $\Delta \Phi=\gamma \boldsymbol{G}_{\mathrm{L}} \delta^{\prime}$. Thus, it will not contribute to the spin echo at the nominal echo time, if the gradient intensity $\boldsymbol{G}_{\mathrm{L}} \delta^{\prime}$ is sufficiently high. Note that for the smallest gradient steps $\boldsymbol{G}_{\mathrm{L}} \delta^{\prime}$ might not be large enough to dephase all destructive coherences and one may observe spin-echo amplitude distortions for low gradient amplitudes $G_{\mathrm{L}}$ (see sec. 4.1.2).

Nonetheless, there are remaining coherence pathways which are completely unaffected by the pulsed gradients of the MRPI pulse sequence. One example is the stimulated echo pathway $\boldsymbol{p}=(0,+1,0, \ldots, 0,-1)$, where $p_{2}=+1$ and $p_{\mathrm{N}}=-1$ correspond to the time intervals when the read gradients are applied. This pathway is stored by the first $180^{\circ}$ rf-pulse in the $z$-direction until it is transformed into detectable signal by the last $180^{\circ} \mathrm{rf}$ pulse, where both rf-pulses partially act as $90^{\circ}$ pulses. Figure 3.3 (left) shows the time domain signal arising from this stimulated coherence pathway with increasing pulsed gradients. The time domain signal was acquired under the influence of the read gradient $\boldsymbol{G}_{\mathrm{R}}$. Note that signal arising from the direct coherence pathway $\boldsymbol{p}_{\text {direct }}$ is attenuated below the noise level due to diffusive attenuation and is therefore absent in the data shown. The spin-echo tops at the nominal echo time (here $t=0$ ) are indicated by red dots. As discussed above, the amplitude of this pathway does not change although the gradient amplitudes are increased. Figure 3.3 (right) shows the signal of the same experiment, but with the last $180^{\circ}$ rf-pulse cycled in phase $\varphi_{4}$ according to the method described in sec. 2.3.3. The stimulated pathway signal is eliminated successfully. Note that residual signals arising from other pathways are still present. 

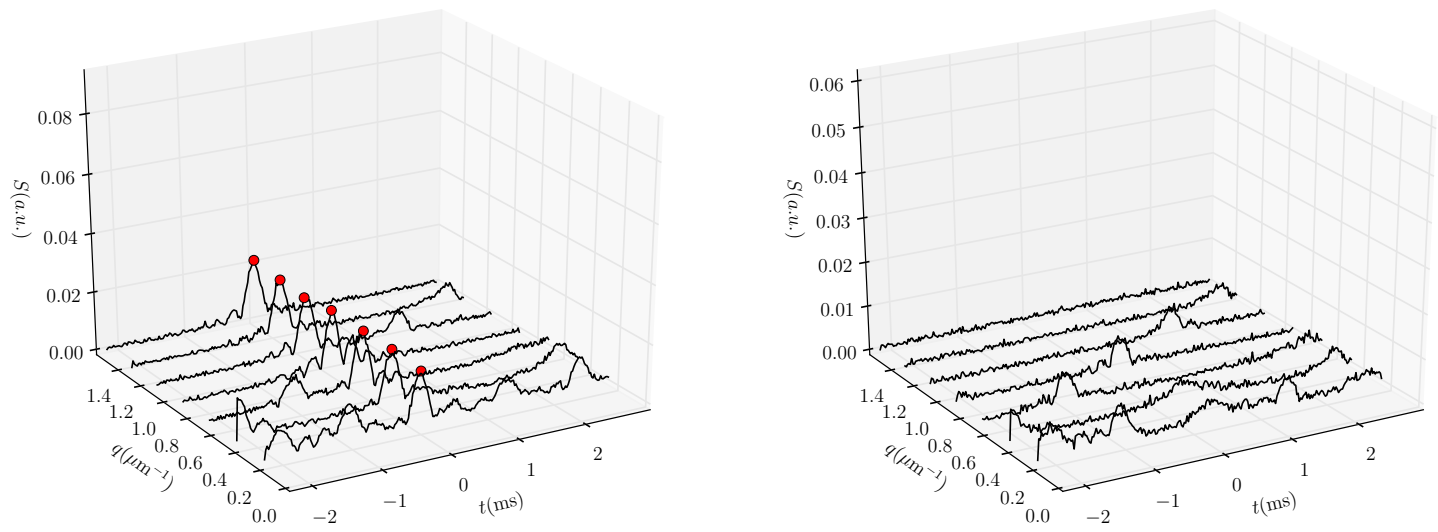

Figure 3.3: Time domain signal of the stimulated echo pathway $\boldsymbol{p}=(0,+1,0, \ldots, 0,-1)$, which constant in amplitude when the pulsed gradient strengths are increased (left). Applying phase cycling to the last $180^{\circ} \mathrm{rf}$-pulse eliminates the signal from this pathway (right). The spin-echo signal of the direct coherence pathway is attenuated below the noise level and is therefor absent in both data sets.

Other unwanted signals were detected in the time domain as shown in fig. 3.3 and the rf-pulses involved in the generation of these coherence pathways were identified by changing the duration of selected $180^{\circ}$ rf-pulses. As discussed in sec. 2.3 .1 the intensity of each pathway depends on the rotation angle generated by the rf-pulses. If the duration of the involved rf-pulse is changed, one may observe a change in the amplitude of the corresponding spin-echo in the time domain.

The phase cycle as shown in tab. 3.1 compensates for the most destructive coherence pathways in our experimental setup. One may notice that it is distinctly different from the CPMG phase cycle as proposed by Meiboom and Gill (1958) and shown in tab. 2.3. The phase cycle proposed here is more akin to the alternating phase cycle as originally proposed by Carr and Purcell (1954), which is due to the unique gradient scheme as utilized with MRPI. The number of phase steps was restricted to 4 steps to keep experiment time within acceptable limits, especially if the pulse sequence is used for multi-dimensional MRPI experiments as will be discussed in sec. 3.1.2 and sec. 3.1.3. Note that especially for low gradient amplitudes this phase cycle is not sufficient to cancel all unwanted coherence pathways. More elaborate phase schemes such as the PIETA approach discussed in sec. 2.3.4 may have to be applied in this case. 
Table 3.1: Phase cycle for the CPMG based MRPI pulse sequence. The number of phase steps was kept at 4 steps to keep experiment time within acceptable limits for multi-dimensional MRPI experiments. Unwanted coherences were identified by analyzing the time domain signal and eliminating echoes arising from unwanted coherence transfer pathways. The rf-phases $\varphi_{\mathrm{i}}$ correspond to the rf-pulses according to fig. 3.2 (a).

\begin{tabular}{ccccc}
\hline \multicolumn{4}{c}{ Pulse phases } & \\
\cline { 1 - 4 }$\varphi_{1}$ & $\varphi_{2}$ & $\varphi_{3}$ & $\varphi_{4}$ & Rec. phase \\
\hline & & & & \\
$x$ & $x$ & $-x$ & $y$ & $-x$ \\
$x$ & $y$ & $-y$ & $x$ & $x$ \\
$x$ & $-x$ & $x$ & $-y$ & $-x$ \\
$x$ & $-y$ & $y$ & $-x$ & $x$ \\
\hline
\end{tabular}

\subsubsection{2d-MRPI}

It is delightful to recognize that the MRPI pulse sequence is in fact an imaging pulse sequence in disguise (Laun et al. (2012)). In doing so, it becomes apparent that the narrow gradient $G_{\mathrm{N}}$ acts similar to the imaging gradient in purely phase encoded MRI. In MRPI the gradient $G_{\mathrm{N}}$ imprints a phase proportional to the distance of the spins to the centre of mass of the pores very similar to phase encoded MRI where the phase gradient imprints a phase proportional to the distance of the spins, however in this case to the center of the gradient system.

This similarity can be exploited to sample $\boldsymbol{q}$ space in whichever experimentally preferred manner. In this work, we applied two approaches known from imaging techniques. These are the 2d-Fourier transform approach discussed in the next section and the Radon-transform approach as will be discussed in sec. 3.1.2.2

\subsubsection{2d-Fourier transform approach}

Based on the similarity of MRPI to phase encoded MRI, a two-dimensional MRPI approach was proposed by Hertel et al. (2013) in which two gradient pulse patterns are stepped independently to sample $\boldsymbol{q}$-space point-by-point on a Cartesian grid. The effective gradient scheme of the two-dimensional version of the MRPI pulse sequence is shown in fig. 3.4. In practice the CPMG based MRPI pulse sequence with alternating $G_{\mathrm{x}}$ and $G_{\mathrm{y}}$ (as shown in fig. 3.2) was employed. Similar to the expression found for 


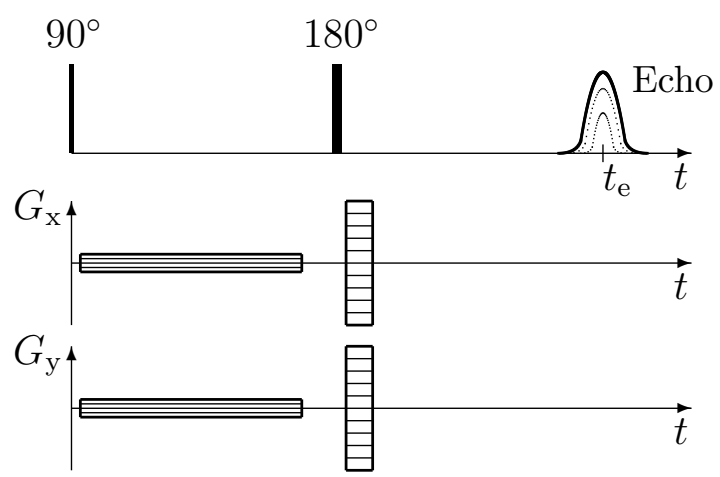

Figure 3.4: 2d-MRPI pulse scheme. Gradients $G_{\mathrm{x}}$ and $G_{\mathrm{y}}$ are stepped independently. In practice the gradient pattern and rf-pulse pattern were replaced with the MRPI pulse pattern (using alternating gradients) shown in fig. 3.2. It was omitted here for clarity.

$\boldsymbol{k}$-space and given by eq. 2.22 , the $2 \mathrm{~d} \boldsymbol{q}$-space signal $S_{0}\left(q_{x}, q_{y}\right)$ is the inverse $2 \mathrm{~d}$-Fourier transform of the $2 \mathrm{~d}$ spin density of the pore space given by

$$
S_{0}\left(q_{x}, q_{y}\right)=\int_{-\infty}^{\infty} \int_{-\infty}^{\infty} \rho_{0}(x, y) \exp \left\{\mathrm{i} 2 \pi\left(q_{x} x+q_{y} y\right)\right\} \mathrm{d} x \mathrm{~d} y
$$

From this it is possible to obtain the average pore image directly by 2 d-Fourier transform of the $2 \mathrm{~d} \boldsymbol{q}$-space data given by

$$
\rho_{0}(x, y)=\int_{-\infty}^{\infty} \int_{-\infty}^{\infty} S_{0}\left(q_{x}, q_{y}\right) \exp \left\{-\mathrm{i} 2 \pi\left(q_{x} x+q_{y} y\right)\right\} \mathrm{d} q_{x} \mathrm{~d} q_{y}
$$

Note that with the pulse sequence shown in fig. $3.4 \boldsymbol{q}$-space is sampled on a discrete Cartesian grid. Therefore, the Fourier transform pair given by eq. 3.2 and eq. 3.3 have to be replaced by the discrete Fourier transform.

\subsubsection{Radon transform approach}

The Radon transform approach may provide an advantage if a set of 1d-MRPI profiles is measured first and the 2d-pore image is subsequently reconstructed. This might be necessary in cases where the sample condition has to be monitored frequently in between the acquisition of 1d-profiles, e.g. to check if any evaporation of the pore fluid has occurred. 
The Radon transform of a function $\rho(r, \theta)$ is given by

$$
P(r, \theta)=\mathcal{R}\{\rho(r, \theta)\}=\int_{-\infty}^{\infty} \rho(r \cos \theta+s \sin \theta, r \sin \theta-s \cos \theta) \mathrm{d} s
$$

where $P(r, \theta)$ is a set of real space projections of the function $\rho(x, y)$ onto the direction $(\cos \theta, \sin \theta)$. The integration proceeds along lines $s$ perpendicular to $(r \cos \theta, r \sin \theta)$. Figure 3.5 shows the Radon transform $P(r, \theta)$ of a 2 d-function $\rho(x, y)$ (resembling a
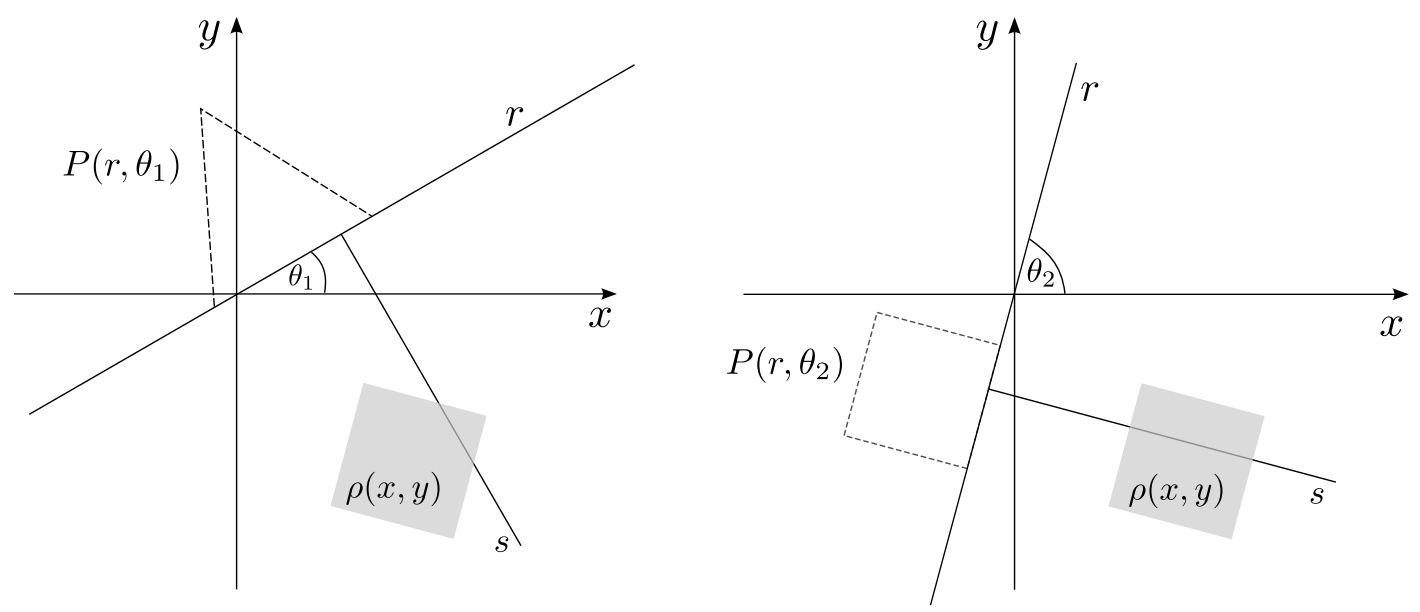

Figure 3.5: Radon transform $P(r, \theta)$ (dashed profile) of a two dimensional function $\rho(x, y)$ for two angles $\theta_{1}$ (left) and $\theta_{2}$ (right).

square) for two exemplary angles $\theta_{1,2}$. The integration in eq. 3.4 proceeds along the line $s$. Thus, the profile $P(r, \theta)$ appears as a triangle when plotting the integrated intensity for all $r$ in case of $\theta_{1}$. While for the second angle $\theta_{2}$ a square profile is obtained. The $\boldsymbol{q}$-space signal is given by the inverse Fourier transform of the profiles $P(r, \theta)$ given by

$$
S(\boldsymbol{q})=\int_{-\infty}^{\infty} P(\boldsymbol{r}, \theta) \exp \{\mathrm{i} 2 \pi q r\} \mathrm{d} r
$$

where $q=|\boldsymbol{q}|=\left(q_{x}^{2}+q_{y}^{2}\right)^{1 / 2}$. If the Radon transform $P(r, \theta)$ is acquired for sufficiently many angles $\theta$, the function $\rho(x, y)$ can be reconstructed by applying an inverse Radon transform also called filtered back-projection. The filtered back-projection is a mathe- 
matical operation given by

$$
\begin{aligned}
\rho_{0}(x, y) & =\int_{0}^{\pi} \int_{-\infty}^{\infty} S(\boldsymbol{q}) \exp \{-\mathrm{i} 2 \pi q r\}|q| \mathrm{d} q \mathrm{~d} \theta \\
& =\int_{0}^{\pi}\left\{\int_{-\infty}^{\infty}\left[\int_{-\infty}^{\infty} P(\boldsymbol{r}, \theta) \exp \{\mathrm{i} 2 \pi q r\} \mathrm{d} r\right]|q| \exp \{-\mathrm{i} 2 \pi q r\} \mathrm{d} q\right\} \mathrm{d} \theta .
\end{aligned}
$$

Thus, using this approach one may acquire radial $\boldsymbol{q}$-space profiles $S(\boldsymbol{q})$ at different angles $\theta$ to the sample. The resulting $q$-space profiles have to be individually Fourier transformed to yield a set of real space profiles $P(r, \theta)$ (projections along the gradient direction) of the sample. Subsequently, a filtered back-projection may be applied to the magnitude data of the real space profiles to obtain the $2 \mathrm{~d}$-image.

\subsubsection{MRPI mapping}

Measuring the spatial distribution of pore sizes is of great relevance in technological applications and for medical diagnosis. One particularly compelling example of the integration of MRPI within the MRI toolbox is the MRPI mapping experiment. It is similar to chemical shift imaging introduced by Mansfield (1984), however here the MRPI $\boldsymbol{q}$-space information is mapped onto MRI images instead of the chemical shift. Figure 3.6 shows the effective gradient pattern of the 1d-MRPI pulse sequence $\boldsymbol{G}^{*}(t)$, where the read gradient $\boldsymbol{G}_{\mathrm{R}}^{*}(t)$ is utilized simultaneously for MRI frequency encoding. A 1d-Fourier transform of the spin-echo for each step in the MRPI gradient strength yields the 1d-profile of the sample with the MRPI $\boldsymbol{q}$-space data mapped onto each pixel of the 1d-profile.

Figure 3.7 shows the effective gradient pulse pattern of the MRPI 2d-mapping experiment. A conventional 2d-MRI pulse sequence typically employs both frequency encoding gradients $\boldsymbol{G}_{\mathrm{R}}^{*}(t)$ and phase encoding gradients $\boldsymbol{G}_{\mathrm{P}}^{*}(t)$ (Bernstein et al. (2004)). In the actual experiment the effective gradient $G^{*}(t)$ is replaced by the CPMG based MRPI alternating gradient pattern as shown in fig. 3.2. In MRPI mapping the read gradient $\boldsymbol{G}_{\mathrm{R}}$ in the beginning and end of the MRPI pulse sequence is not only used for establishing a well defined echo condition, but for frequency encoding in read direction as 


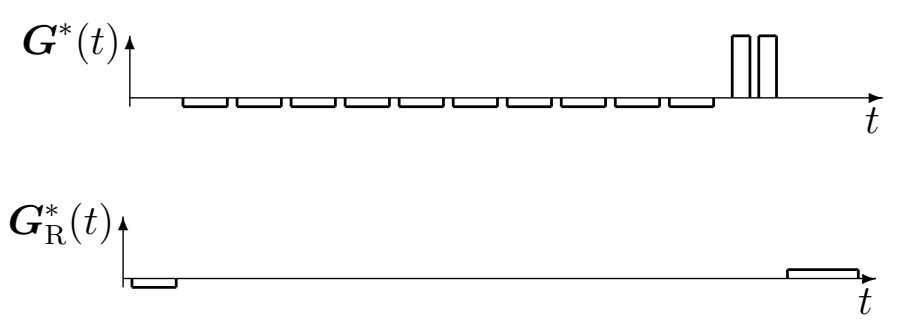

Figure 3.6: MRPI 1d-mapping pulse sequence for acquisition of the spatial distribution of pore shapes and sizes mapped onto 1d-MRI images. The read gradient $G_{\mathrm{R}}^{*}(t)$ is utilized for MRI frequency encoding for each step in the MRPI gradient strength $G^{*}(t)$. Effective gradients of the MRPI part $G^{*}(t)$ are stepped (steps are not shown).

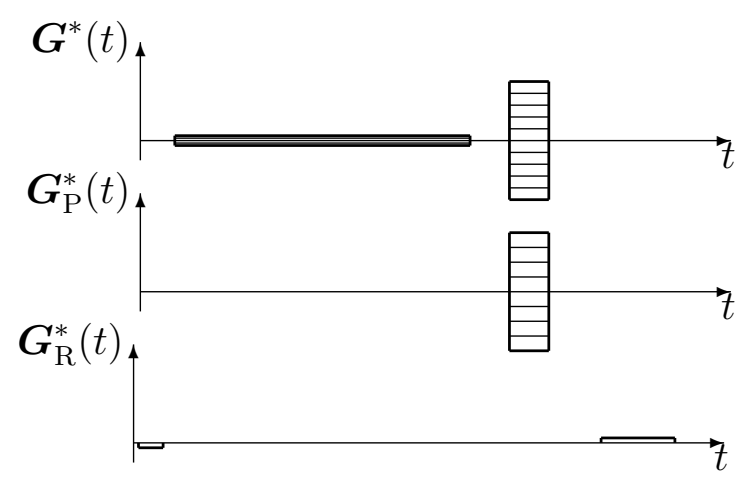

Figure 3.7: MRPI 2d-mapping pulse sequence for acquisition of the spatial distribution of pore shapes and sizes mapped onto MRI images. Effective gradients of the MRPI part $G^{*}(t)$ are stepped for each step in the phase gradient $G_{\mathrm{P}}^{*}(t)$. The read gradient $G_{\mathrm{R}}^{*}(t)$ yields the second dimension of the MRI image.

well. Additionally, a phase gradient $\boldsymbol{G}_{\mathrm{P}}$ is stepped independently to provide the phase encoding of the MRI image. Thus, the experiment proceeds by sampling $\boldsymbol{q}$-space for each step in the phase gradient $\boldsymbol{G}_{\mathrm{P}}$. A two-dimensional MRI image is acquired and the 1d-MRPI signal is encoded in the third dimension.

\subsection{Challenges imposed by NMR hardware limitations}

\subsubsection{NMR hardware used}

The experiments for this thesis were performed on a Bruker Avance 400 spectrometer operating at a proton resonance frequency of $400 \mathrm{MHz}$. Imaging capabilities needed for 
the generation of the gradient pulses were provided by a Bruker micro-imaging system. This system consisted of a Bruker Micro 2.5 imaging probe, which provides gradients in all three Cartesian directions with gradient strengths of $24.2 \mathrm{mTm}^{-1} \mathrm{~A}^{-1}$. The inductance of the gradient coils was $L_{\text {coil }} \leq 100 \mu \mathrm{H}$ and the resistance was $R \leq 400 \mathrm{~m} \Omega$. Three Bruker GREAT 60 gradient amplifiers were utilized each having a maximum current output of $60 \mathrm{~A}$ at a DAC resolution of 16 bit. At the maximum current output of $\pm 60 \mathrm{~A}$ the gradient system provided gradient strengths up to $G_{\max }= \pm 1.45 \mathrm{Tm}^{-1}$. The minimum gradient pulse rise times, due to the maximum voltage of the gradient amplifier of $U_{\max }= \pm 100 \mathrm{~V}$, were between $50 \mu$ s and $150 \mu$ s depending on the gradient coil inductance.

The utilized rf-coil had a birdcage coil design and allowed for a maximum outer diameter of the sample of $O D=1 \mathrm{~cm}$. Typical rf-pulse durations were $t_{90^{\circ}}=12.5 \mu \mathrm{s}$ and $t_{180^{\circ}}=25 \mu$ s when utilizing ${ }^{1} \mathrm{H}$ as the NMR active nuclei in a distilled $\mathrm{H}_{2} \mathrm{O}$ sample.

\subsubsection{Resolution limit of the gradient amplifier}

In contrast to conventional PGSE NMR experiments, the MRPI pulse sequence contains a long weak gradient $G_{\mathrm{L}}$. Due to the typical duration of the long gradient $\delta_{\mathrm{L}}$ which may be on the order of several $100 \mathrm{~ms}$, there may be gradient mismatches due to the finite DAC resolution of the gradient amplifier. Figure 3.8 shows on the example of the unaltered MRPI pulse sequence how a small gradient uncertainty $\Delta G^{\text {min }}$ may cause a large shift in the spin-echo position $\Delta t$ when acquired during application of a read gradient $\boldsymbol{G}_{\mathrm{R}}$. With the digital resolution of the DAC of the amplifier of 16 bit there

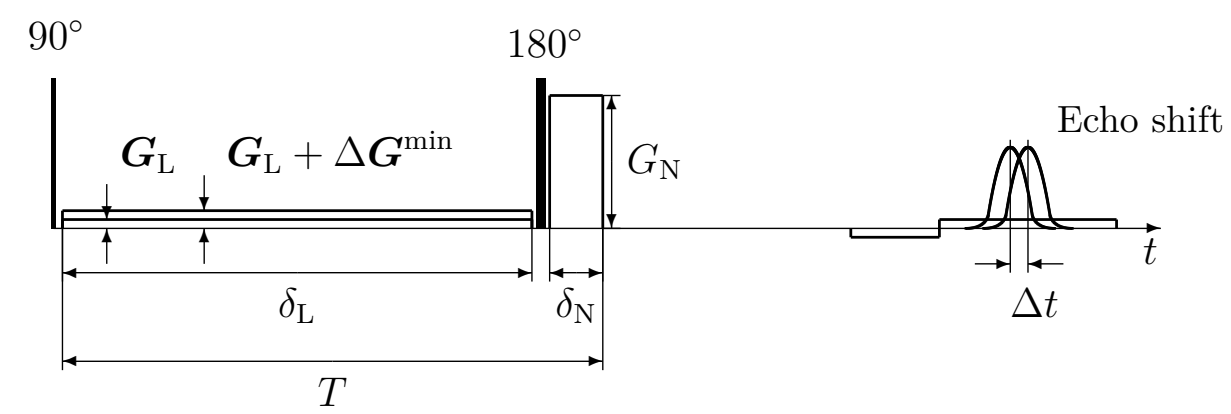

Figure 3.8: Spin echo shift due to gradient mismatch caused by the finite resolution of the gradient amplifier. 
are effectively $2^{15}$ current steps ( 1 bit is reserved to determine the polarity). Thus the minimal gradient step is given by $\Delta G^{\mathrm{min}}=1.45 \mathrm{Tm}^{-1} / 2^{15}=4.4 \times 10^{-5} \mathrm{Tm}^{-1}$. In case the gradient amplitude of the long gradient is missed by half a bit this value $\Delta G^{\text {min }}$ has to be multiplied by the ratio $\delta_{\mathrm{L}} / \delta_{\mathrm{N}}$ to reflect the gradient mismatch. At a ratio of $\delta_{\mathrm{L}} / \delta_{\mathrm{N}}=100-1000$ this value lies in the tenths of milli tesla regime and can lead to serious additional spin echo attenuation (Kärger et al. (1988), Price (1998)) in absence of a read gradient or a large time shift $\Delta t$ when a read gradient is applied. For this reason, we implemented a program to determine the long gradient amplitude $G_{\mathrm{L}}$ first with amplitudes matching to the actual values as output by the DAC and with a precision up to the digital step. Subsequently, the narrow gradient amplitude $G_{\mathrm{N}}$ was automatically calculated from this information.

Note that it may be more convenient to set the narrow gradient strength first to determine the resolution and the field of view of the MRPI experiment. The described procedure above therefore represents an additional step, which was automatically performed by the pulse program.

\subsubsection{Further experimental precautions}

Ramping of the gradient pulses may be crucial for the CPMG based MRPI pulse sequence, since a large number of weak gradient pulses are utilized which are balanced by two intense gradient pulses. In case of the presence of eddy currents or other gradient amplitude distortions their effect will be multiplied by the overall number of gradient pulses.

Figure 3.9 (a) shows a pulsed field gradient shape as it may look like if no ramping of the current output is applied. The gradient amplitude may be distorted in the time intervals of the rising and falling edges of the gradient shape. The exponential rise and fall of the gradient amplitude is determined by the inductance of the gradient coils $L_{\text {coil }}$ and the finite voltage range $U_{\max }$ of the gradient amplifier. Furthermore, eddy currents may be induced during switching of the gradients which scale with the slew rate $\mathrm{d} I / \mathrm{d} t$ (Price (1998)). Eddy currents may lead to additional artifacts if they extend until the application of an rf-pulse, since they may change the selectivity of the rf-pulses (Price 
a)

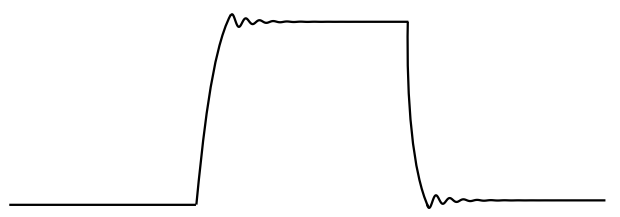

b)

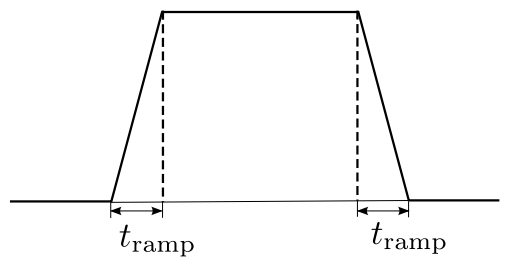

Figure 3.9: Pulsed field gradient shape if no ramping of the gradient amplitude is applied. Eddy currents and noise from the main power supply may occur (a). The desired pulsed field gradient shape may be obtained by ramping the current in the gradient coil during a time interval $t_{\text {ramp }}(\mathrm{b})$.

(1998)). In contrast, fig. 3.9 (b) shows a shaped gradient when the gradient shape is controlled by ramping the current during a time $t_{\text {ramp }}$. This way the desired gradient shape can be obtained. In this work we ramped the current by digital stepping with 10 steps and a total ramp time of $t_{\mathrm{ramp}}=0.1 \mathrm{~ms}$. Hence, the voltage drop $U$ across the gradient coils as given by $U=I R+L_{\text {coil }} \mathrm{d} I / \mathrm{d} t$ at a maximum current of $I=60 \mathrm{~A}$ does not exceed $U= \pm 84 \mathrm{~V}$ and is therefore well below the maximum voltage range of the gradient amplifier of $U_{\max }= \pm 100 \mathrm{~V}$. In order to prevent residual eddy currents during application of the rf-pulses a minimum separation time of $0.25 \mathrm{~ms}$ after each gradient pulse was implemented in the pulse program.

\subsection{Samples}

This section presents the utilized samples for calibration of the MRPI pulse sequence as well as the phantom samples used for proving the general applicability of MRPI. The phantom samples were custom made to provide well defined porous systems. The triangular capillaries presented in sec. 3.3.3 were produced in collaboration with the Photon Factory at Auckland University.

\subsubsection{Calibration samples}

The MRPI pulse sequences presented in the previous sections were newly developed and all pulse programs were written as part of this thesis. Therefore, it was crucial to apply 
these sequences first to well known calibration samples. Two different calibration samples were utilized which contained liquids of known self-diffusion coefficients. Sample CAL1 consisted of a cylindrical sample tube of inner diameter $I D=0.8 \mathrm{~cm}$ filled with distilled $\mathrm{H}_{2} \mathrm{O}$. However, a second sample (CAL2) was needed for the case of calibrating the pulse sequence up to the highest gradient strengths available. For this purpose, a substance with low self-diffusion coefficient was needed. We chose poly-dimethyl-siloxane (PDMS) with a molecular weight of $M_{\mathrm{w}}=36000 \mathrm{gmol}^{-1}$ in a sample tube of inner diameter of $I D=0.8 \mathrm{~cm}$. Manufacturer information about the polydispersity of the PDMS sample was not available, but preliminary PGSE NMR self-diffusion measurements suggest a bimodal distribution of chain lengths.

\subsubsection{Cylindrical glass capillaries as phantom samples}

Cylindrical glass capillaries with inner diameters in the micro-metre range were utilized in a number of recent studies involving PGSE NMR and NMR methods to extract pore sizes and other parameters. Examples of recent contributions utilizing such samples include Bar-Shir et al. (2008), Shemesh et al. (2009), Benjamini and Nevo (2013), Álvarez et al. (2013) and Shemesh et al. (2012). This model porous system is ideally suited to study the influence of the various parameters of the MRPI pulse sequence, since the domain is straightforward to simulate and the inner glass surface leads to a negligible wall relaxation.

The capillaries utilized in this study were commercially available cylindrical glass capillaries (Polymicro Inc.) with an inner capillary radius of $L=10 \pm 1 \mu \mathrm{m}$. These particular capillaries consist of a silica core coated with polyimide. Figure 3.10 (left) shows schematically an extruded silica capillary. The outer diameter of the capillaries including the coating was $O D=360 \mu \mathrm{m}$. The polyimide coating is giving the capillaries some flexibility and allows them to be wound on spools for distribution. Hence, one has to cut the several metre long capillary into pieces of the desired length using a sharp knife. For this study a total of 470 capillaries were cut with a length of $5 \mathrm{~cm}$ each. Subsequently, the capillaries were submerged in distilled $\mathrm{H}_{2} \mathrm{O}$ for filling by capillary forces. The bath was heated to the boiling point in order to remove residual gas trapped 

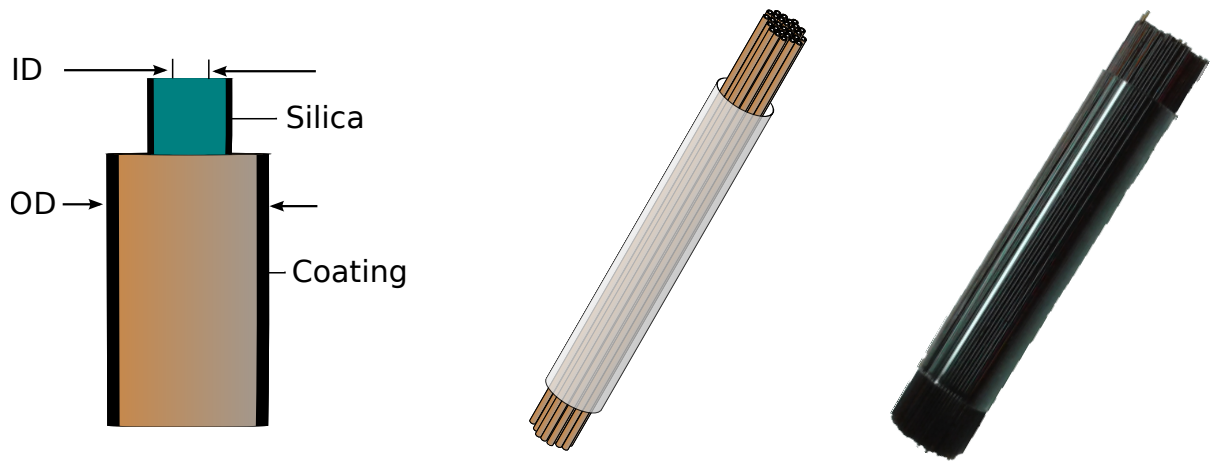

Figure 3.10: Sketch of the cylindrical micro-capillaries used to assemble the NMR phantom (left). The final sample, which consisted of 470 fused silica capillaries stacked in a glass cylinder (middle). Photo of the final sample CYL1 (right).

in the capillaries. After removal of the capillaries from the water bath, excess water in the inter capillary spaces was removed by blotch-drying. Figure 3.10 (right) shows the final capillary stack, which was designed by packing all capillaries in a glass sample tube.

A second sample containing two different capillary diameters was prepared for the MRPI mapping experiments as discussed in sec. 4.4. A sketch of the transverse crosssection through the sample is shown in fig. 3.11. Note that the sketch is not to scale and many more capillaries were present in the sample. This sample was prepared by creating two compartments by inserting a polymer sheet in the middle of the glass sample tube. Both compartments were filled with capillaries of cylindrical shape, however their

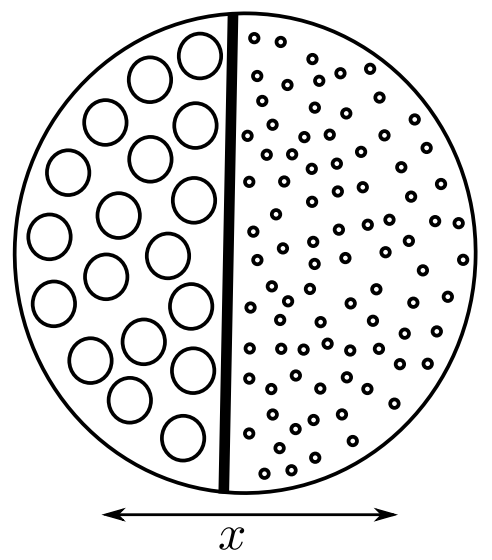

Figure 3.11: Sketch of the capillary sample CYL2 with two compartments of different capillary radii (not to scale). 
radii for the two compartments differed and were $L_{1}=10 \pm 1 \mu \mathrm{m}$ and $L_{2}=5 \pm 1 \mu \mathrm{m}$ respectively. The final sample with two compartments appeared the same from the outside as the sample with only one size of capillaries and thus no photo is shown of the assembly. The sample containing capillaries of only one type with $L=10 \pm 1 \mu \mathrm{m}$ is called CYL1 in this work. The sample containing two capillary sizes of $L_{1}=10 \pm 1 \mu \mathrm{m}$ and $L_{2}=5 \pm 1 \mu \mathrm{m}$ is called CYL2.

Upon drying the filled stacks were imaged using an MRI imaging (Bruker Paravision 5.0) sequence to check for correct filling and to exclude the possibility of any water presence in the inter-capillary spaces. The pulse sequence used was Flash with a maximum resolution of $26 \mu \mathrm{m} \times 26 \mu \mathrm{m}$. Figure 3.12 shows an image of the spin density as observed using a $2 \mathrm{~mm}$ thick slice perpendicular to the symmetry axis of the cylinders. The filled capillaries appear as white dots. Indicated is the distance between nearest

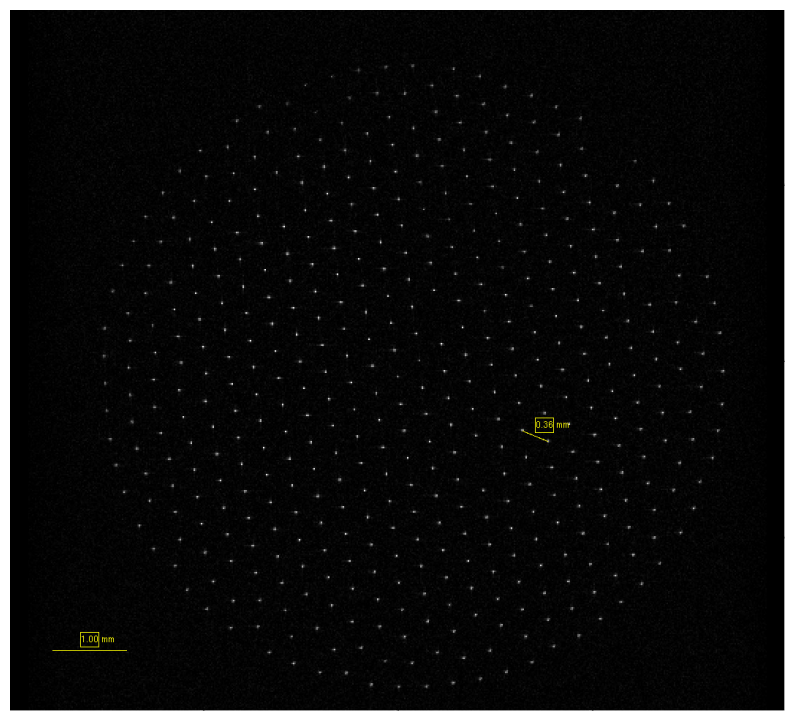

Figure 3.12: MRI image of the filled capillaries. Paravision Flash pulse sequence with a resolution of $26 \mu \mathrm{m} \times 26 \mu \mathrm{m}$. Axial $2 \mathrm{~mm}$ thick slice in the middle of the filled capillaries. Filled capillaries appear as white dots.

neighbouring capillary centers, which is $0.36 \mathrm{~mm}$ in agreement with the diameter of each capillary including the silicate core and the polymer coating. Note that the image shown in fig. 3.12 has been acquired with the highest resolution obtainable for MRI images with our gradient system. No MRI image of the sample CYL2 has been included here, since the MRI images of the samples CYL1 and CYL2 were not discernible. The fact that 
the capillaries appear only as white dots without any information about their shape shows once more the necessity for alternative techniques such as MRPI to obtain higher resolution images.

\subsubsection{Hemi-equilateral triangular capillaries as phantom samples}

The form factor of point symmetric pore shapes such as a cylinder is a real function and the imaginary part is zero. However, the concept of MRPI promised to return also non-zero imaginary parts with arbitrary phases between the real and imaginary part. Thus, a non-pointsymmetric pore system was needed to prove the general applicability of the MRPI technique. Therefore, one major challenge of this work was to produce a suitable phantom sample with non-pointsymmetric pore shapes on the micro-metre scale. For this work, we chose a hemi-equilateral triangular shape, since its MCF parameters necessary for MRPI simulations have recently been calculated by Laun et al. (2012). The requirements for the final sample are high since the pore shape dimensions have to be of high precision to prevent washing out of the average pore image. Additionally, all capillaries have to be prepared with the same orientation such that the average pore image resembles the underlying pore shape.

\subsubsection{Principle of excimer laser dragging}

The required set of capillaries (sample TRI) was created in collaboration with the Photon Factory at the University of Auckland. The method used was microgroove pattern machining by excimer laser dragging. Figure 3.13 shows schematically the process of excimer laser dragging as published by Hocheng and Wang (2008). The excimer laser is guided perpendicular onto the mask containing the desired pattern. When the laser impinges on the triangular mask, the laser beam will go through the transparent portion of the mask onto the work piece surface and ablate the work piece. The pattern may be machined with macroscopic dimensions into the mask and it will be de-magnified by the projection lens to the desired dimension of the pattern on the work piece.

The laser ablates material from the work piece with an ablation depth proportional to the exposure time and the fluence $F$ of the laser. As schematically shown in fig. 3.14, 


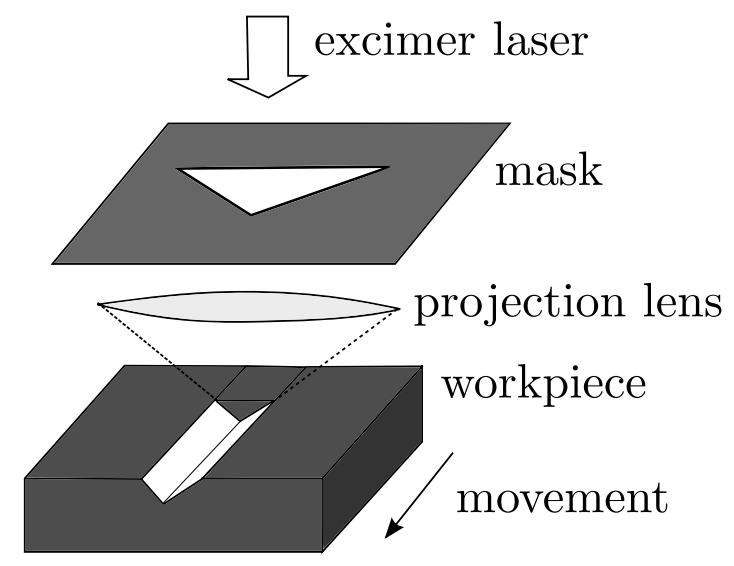

Figure 3.13: Scheme of microgroove pattern machining by excimer laser dragging. Adapted from Hocheng and Wang (2008).
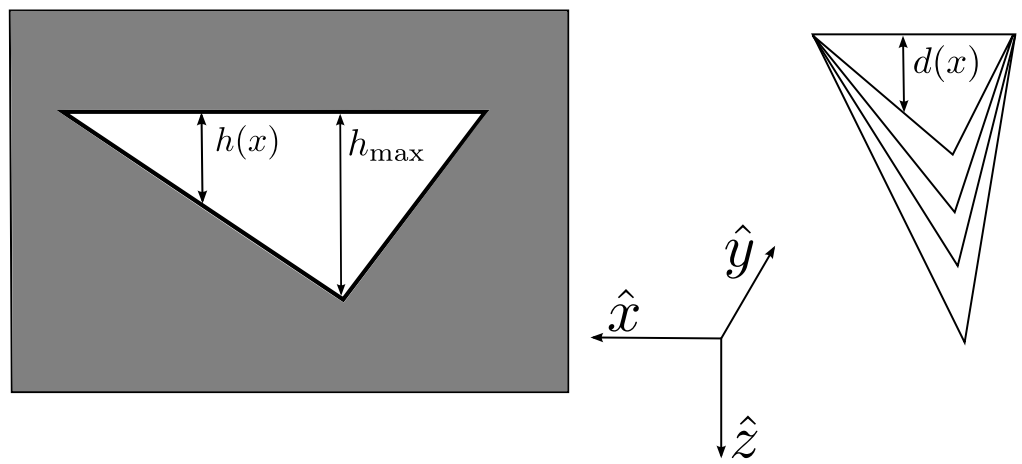

Figure 3.14: Scheme of the height of the mask and the depth of the groove. Adapted from Hocheng and Wang (2008)

the depth of the groove $d(x)$ in the work piece is therefore proportional to the height of the mask pattern in the scanning direction $h(x)$ and it is inversely proportional to the movement velocity $v$. Thus, the depth is given by

$$
d(x) \propto \frac{h(x) F}{v} .
$$

Additionally, the width of the grooves in the work piece $L$ will be given by the width of the mask pattern $W$ divided by the de-magnification factor. 


\subsubsection{Production of triangular capillary arrays}

The triangular mask was machined into chrome on quartz using a $800 \mathrm{~nm} 110 \mathrm{fs}$ pulsed laser $\left(\right.$ Coherent ${ }^{\circledR}$ Legend elite). The extensions of the mask were $W=400 \mu \mathrm{m}$ and $h_{\max }=1200 \mu \mathrm{m}$. Using this mask, a $248 \mathrm{~nm}$ nanosecond pulsed laser (Coherent ${ }^{\circledR}$ Xantos XS) was utilized at a fluence of $F=3 \mathrm{Jcm}^{-2}$ to machine hemi-equilateral triangular channels into sheets of amorphous poly-carbonate (Makrofol ${ }^{\circledR}$ ). The quartz mask was mounted at $20 \times$ de-magnification to yield a characteristic width of the channels of $L=20 \mu \mathrm{m}$. The length of the channels in dragging direction was $l=5 \mathrm{~cm}$. Figure 3.15 shows schematically a part of an array of closed capillaries with a channel separation of $80 \mu \mathrm{m}$, while the insert in fig. 3.15 shows schematically the dimensions of the channel cross section. Closed hemi-equilateral channels were created by bonding a flat poly-
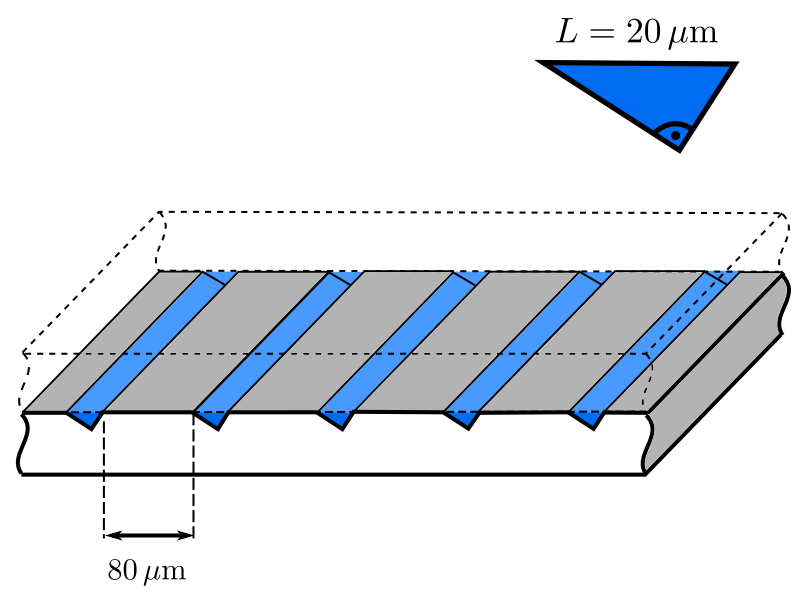

Figure 3.15: Sketch of hemi-equilateral triangular pores and their alignment into an ordered array. The characteristic width of $L=20 \mu \mathrm{m}$ is indicated for one channel cross-section (top). The channels were machined into amorphous polycarbonate sheets. The separation between channels was $80 \mu \mathrm{m}$. Closed hemi-equilateral channels were created by bonding a flat polycarbonate sheet on top of the array (indicated as dotted line).

carbonate sheet on top of the array (indicated as dotted line in fig. 3.15). Bonding of two polycarbonate sheets was performed by exposing the surface of the sheets to Dichloromethane (DCM) following a procedure published by Ogonczyk et al. (2010). After machining the channels, a blank polycarbonate sheet was exposed to DCM vapour for three minutes. For this purpose, the sheet rested on a glass microscope slide above a covered petri dish filled with DCM. The exposed surface of the sheet was applied to 


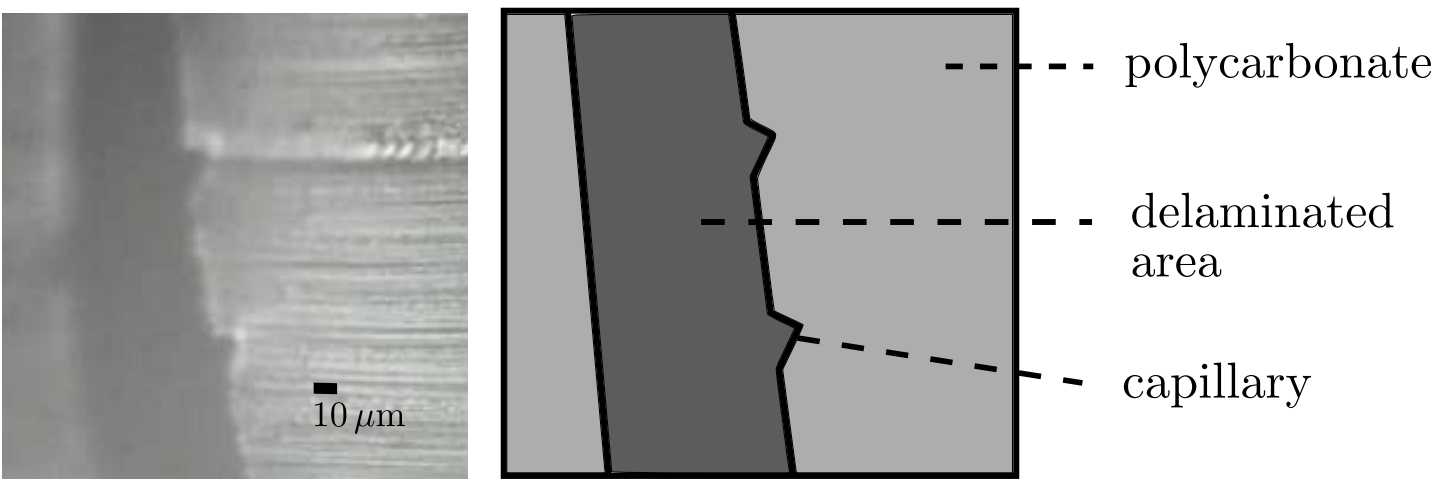

Figure 3.16: Optical microscope image of the channel cross-section for two exemplary triangular capillaries. The flat poly-carbonate sheet de-laminated during cutting of the stack (dark grey region) and the channels appear to be open (left). The schematic drawing shows the different regions of the polycarbonate and the de-laminated region (right). The intact samples used for our MRPI experiments contained only closed channels as verified by MRI images of the sample cross section of samples filled with water.

the featured side of a machined sheet to bond in the flat regions of the machined sheet. This way the machined channels were capped by the flat surface creating sets of closed capillaries. The edges of the flat polycarbonate sheet which is closing the channels are indicated as dashed lines in fig. 3.15. Following this procedure we produced chips with two rows of channels and each row containing 40 channels. Thus, each chip contained 80 microchannels.

Figure 3.16 shows an optical microscope image of the cross-section of two exemplary channels. For this image the stack had to be cut with a sharp knife, which led to a delamination of the flat sheet and thus the channels appear to be open (dark grey region in fig. 3.16). Filling with distilled water was carried out by submersion of the whole chips into a water bath. Subsequently, vacuum was applied over the water phase to remove residual air in the channels by degassing. After sufficient degassing had occurred the vacuum was removed to allow the filling of the capillaries with distilled water. In order to maximize signal-to-noise for the NMR experiments, a total of 4 of these filled chips were stacked in a sample tube of diameter $d=1 \mathrm{~cm}$. A sketch of the stack can be seen in fig. 3.17.

The final sample contained a total of 320 micro-channels, since each chip contained 2 rows of channels with 40 channels per row. The aforementioned sample was characterized 


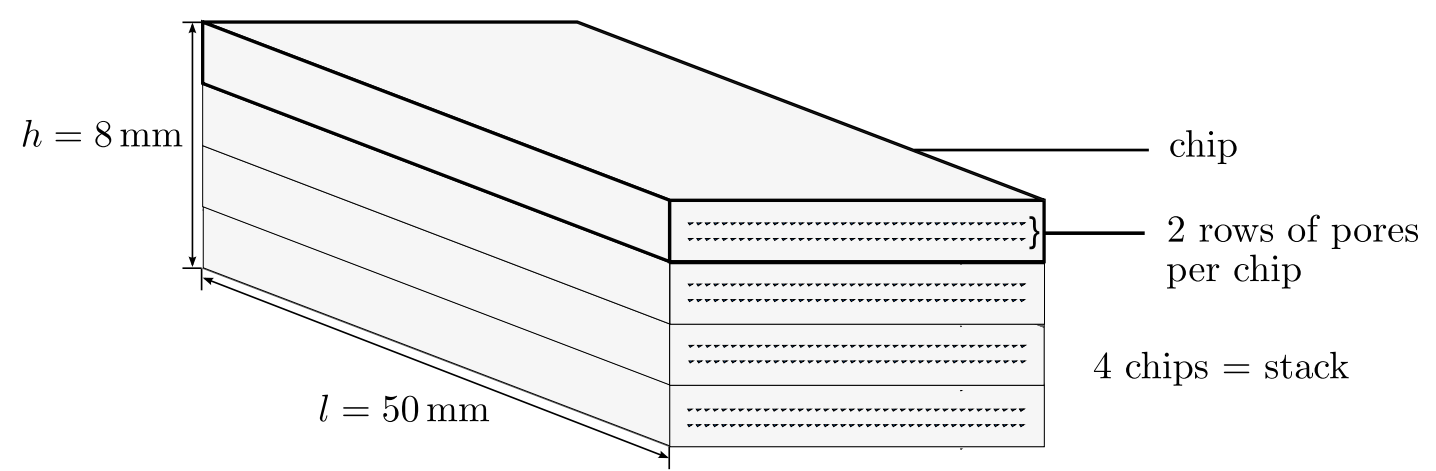

Figure 3.17: Sketch of the final stack of polycarbonate chips. Each chip contained two rows of triangular channels. In total 4 chips were stacked leading to a final length of the assembly of $l=50 \mathrm{~mm}$ and a height of $h=8 \mathrm{~mm}$.

using standard MRI images as shown in fig. 3.18 to ensure that signal was only coming from inside the pores and that the pores were isolated from each other. One may

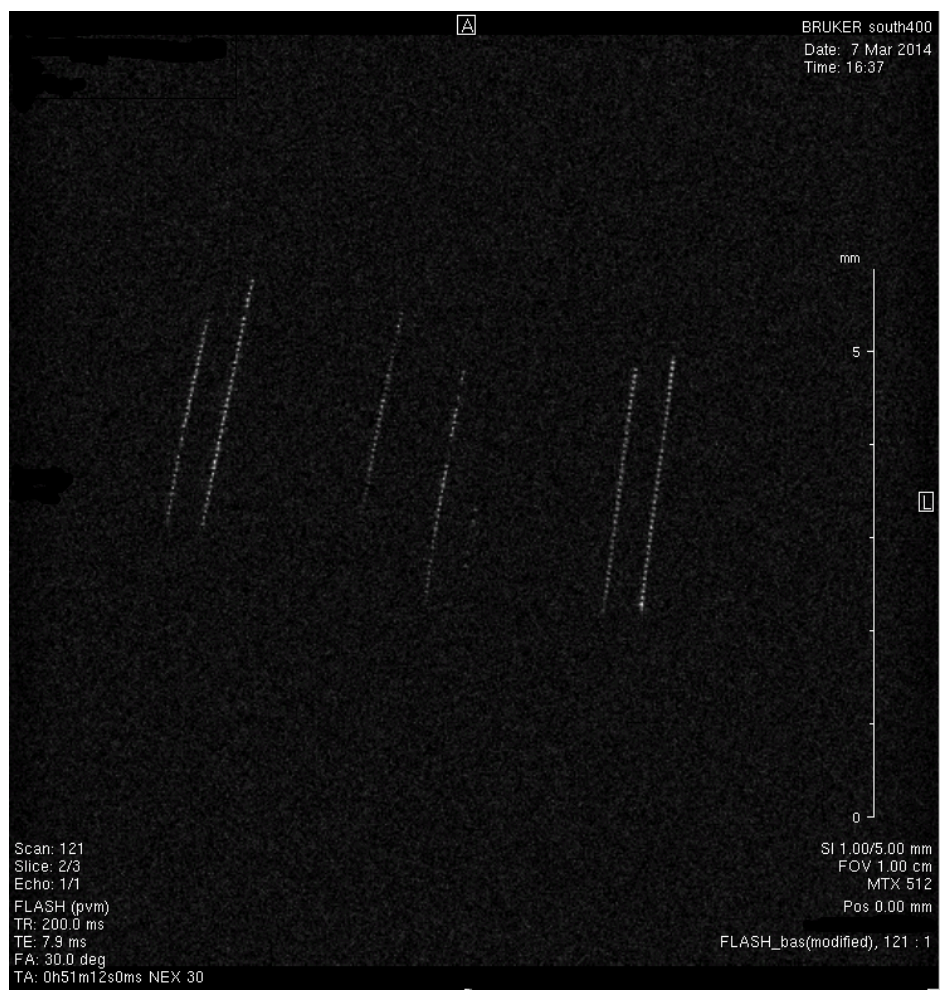

Figure 3.18: MRI image of the filled triangular capillaries. 4 chips are present in the sample with 2 layers of channels each. However, only 6 layers (white lines) can be observed in the MRI image. The white dots are the filled channels. Close examination shows that the white dots are separated with regions free of signal, indicating that the channels are isolated from each other. 
notice that there are 6 white lines which can be discerned in fig. 3.18, although 8 lines are expected (4 chips with 2 layers of channels each). The absence of these two lines indicates that the channels may have been closed during gluing. Close examination shows that the white dots are separated with regions free of signal, indicating that the channels are isolated from each other. 


\section{Results and discussion}

\subsection{Free diffusion}

\subsubsection{Calibration}

In order to test whether the given NMR setup is suitable to apply long weak gradients, which are refocused by short intense gradients we measured the unrestricted selfdiffusion of sample CYL1 whose self-diffusion coefficient is known from the literature. Self-diffusion coefficients of distilled water for different temperatures are given with a high precision by Mills (1973), Holz and Weingärtner (1991), Holz et al. (2000) and Tofts et al. (2000) and are thus well suited to calibrate PGSE NMR experiments. The calibration procedure relies on the exponential dependence of the spin-echo amplitude $E$ on the self-diffusion coefficient $D$ and the $b$-value as described in sec. 2.2.1. The $b$-value contains all experimental parameters and is given by the double time integral eq. 2.33. Thus, if the $b$-value is known for a specific pulse sequence, the fit of the spin-echo attenuation $E(b)$ has to yield a self-diffusion coefficient which agrees with the literature value if the experimental setup is working as expected. The $b$-value for the PGSE NMR pulse sequence with two narrow gradient pulses with $\Delta \gg \delta$ such that $\Delta \approx T$ is given by $b_{\mathrm{PGSE}}=(\gamma G \delta)^{2} T$.

In the case of the unaltered long-narrow PGSE NMR pulse scheme (as shown in fig. 2.13 (b)) a weaker signal decay is expected than for the diffusive-diffraction PGSE NMR pulse sequence with two narrow gradients. The $b$-value for long-narrow PGSE NMR as found by evaluating eq. 2.33 in the limit $T \gg \delta$ is given by $b_{\mathrm{LN}}=(\gamma G \delta)^{2} T / 3$ 
and thus one has

$$
b_{\mathrm{LN}}=\frac{b_{\mathrm{PGSE}}}{3} .
$$

Hence, the spin-echo attenuation of the unaltered long-narrow PGSE NMR experiment is smaller by a factor of three as compared to a PGSE NMR experiment with the same gradient scheme duration $T$ and for a given gradient amplitude $G$. Figure 4.1 shows a comparison for the spin echo attenuation of the unaltered long-narrow PGSE NMR pulse sequence (squares) and the diffusive-diffractive PGSE NMR pulse sequence (circles) with the sample CAL1 at a temperature of $293 \mathrm{~K}$. In both cases a spin-echo rf-pulse scheme (see sec. 2.1.3) was utilized consisting of a leading $90^{\circ}$ and a refocusing $180^{\circ}$ rf-pulse. The gradient parameters were $\delta=2 \mathrm{~ms}, \Delta=50 \mathrm{~ms}$ for the diffusive-diffraction PGSE NMR experiment and $\delta_{\mathrm{L}}=50 \mathrm{~ms}, \delta_{\mathrm{N}}=2 \mathrm{~ms}$ for the unaltered long-narrow PGSE NMR experiment. The echo time for both pulse sequences was $t_{\mathrm{E}}=2 \tau=100 \mathrm{~ms}$. The self-

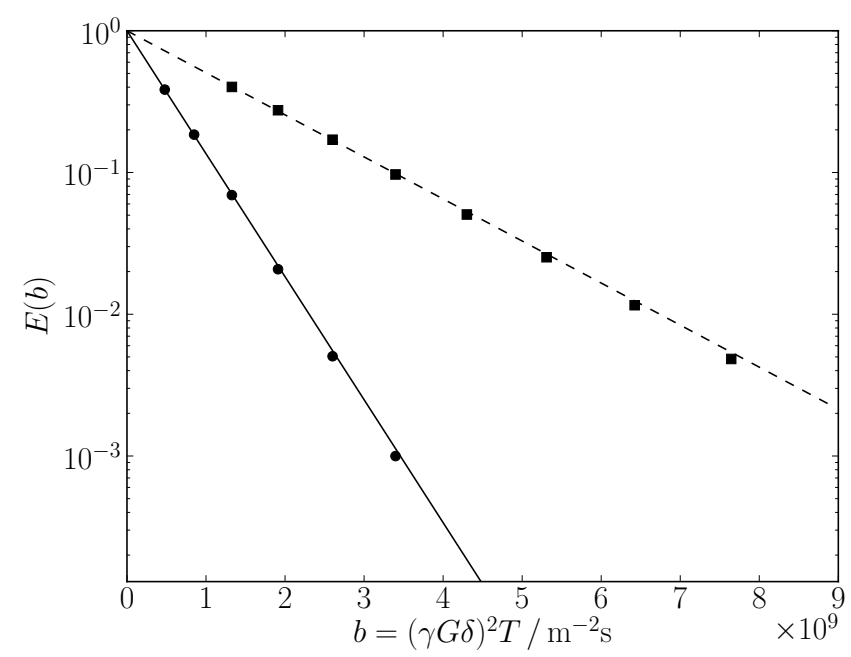

Figure 4.1: Comparison of spin-echo attenuation of the unaltered MRPI experiment (squares) versus the diffusive-diffraction PGSE NMR experiment (circles) for sample CAL1. The slopes of the fits for the MRPI experiment (dashed line) and the diffusive-diffraction PGSE NMR experiment (solid line) yield a ratio of 0.34 close to the expected value of 0.33 .

diffusivities were fitted for both data sets and yielded $D_{\mathrm{LN}}=2.1 \pm 0.1 \times 10^{-9} \mathrm{~m}^{2} \mathrm{~s}^{-1}$ via fit against $b_{\mathrm{LN}}$ (dotted line in fig. 4.1) and $D_{\mathrm{PGSE}}=2.0 \pm 0.1 \times 10^{-9} \mathrm{~m}^{2} \mathrm{~s}^{-1}$ via fit against $b_{\mathrm{PGSE}}$ (solid line in fig. 4.1). Thus, the results verify the validity of eq. 4.1 since the two 
self-diffusivities agree with each other within experimental uncertainties.

\subsubsection{1d-MRPI calibration}

In case the MRPI pulse sequence is applied to measure self-diffusion in an unrestricted sample exhibiting Gaussian diffusion, one may find the $b$-value by evaluating eq. 2.33. The corresponding $b$-value for the 1d-MRPI pulse sequence based on CPMG applying $\int_{0}^{T} G^{*}(t) \mathrm{d} t=0$ and with two narrow gradient pulses is given by

$$
b=\frac{\left(\gamma G_{\mathrm{N}} 2 \delta^{\prime}\right)^{2}}{3}\left[\delta^{\prime}\left(N_{\mathrm{L}}+2\right)+\delta_{\mathrm{s}}\left(\frac{2 N_{\mathrm{L}}^{2}+3 N_{\mathrm{L}}+1}{2 N_{\mathrm{L}}}+\frac{3}{4}\right)\right]
$$

where the parameters $G_{\mathrm{N}}, \delta^{\prime}$ and $N_{\mathrm{L}}$ are defined in fig. 3.2. The duration $\delta_{\mathrm{s}}$ is the separation between the gradient pulses, i.e. the time from the end of one gradient pulse until the leading edge of the next gradient pulse. Equation 4.2 reduces to eq. 4.1 in the limit of $\delta_{\mathrm{s}} \rightarrow 0$ and $\delta^{\prime}\left(N_{\mathrm{L}}+2\right)=T$. The effect of the gradient pulse ramps is partially accounted for by adding one ramp time to the gradient pulse duration $\delta^{\prime}$.

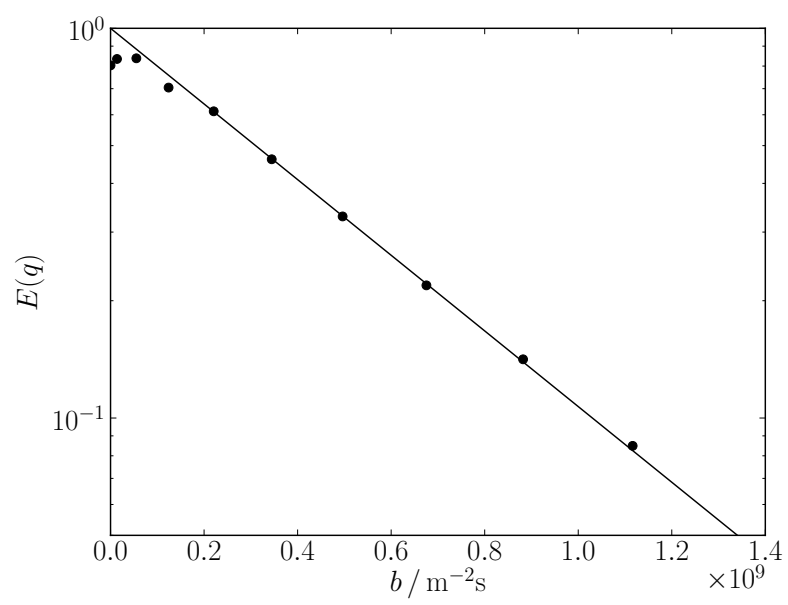

Figure 4.2: Experimental data obtained by applying the 1d-MRPI pulse sequence to measure free diffusion of $\mathrm{H}_{2} \mathrm{O}$ in sample tube (dots). The fit of the data with eq. 4.2 yields a self-diffusion coefficient of $D=2.2 \pm 0.2 \times 10^{-9} \mathrm{~m}^{2} \mathrm{~s}^{-1}$ (solid line).

Figure 4.2 shows the result when applying the 1d-MRPI pulse sequence to measure unrestricted self-diffusion in sample CAL1 at a temperature of $T_{\mathrm{K}}=293 \mathrm{~K}$. The param- 
eters of the MRPI pulse sequence were $N_{\mathrm{L}}=40, \delta^{\prime}=2 \mathrm{~ms}, 2 \tau=2.5 \mathrm{~ms}, \delta_{\mathrm{s}}=0.5 \mathrm{~ms}$ and $G_{\mathrm{N}}^{\max }=0.175 \mathrm{Tm}^{-1}$. A fit of the experimental data yields a self-diffusion coefficient of $D=2.2 \pm 0.2 \times 10^{-9} \mathrm{~m}^{2} \mathrm{~s}^{-1}$ within experimental error of the literature value of $D=2.0 \times 10^{-9} \mathrm{~m}^{2} \mathrm{~s}^{-1}$ (Holz and Weingärtner (1991)).

The larger value obtained for the self-diffusion coefficient may be caused by not taking into account the extra term in the $b$-value arising from the gradient ramp times, a correction term well known in PGSE NMR diffusion experiments and published by Price and Kuchel (1991). In this work we chose to ignore this term, since we were foremost concerned with obtaining a Gaussian attenuation for the case of free diffusion as well as a stable spin-echo in the time domain with each pulsed gradient step. The spin echo attenuation shows a depression for the spin-echo amplitude of the first four data points. This phenomenon is more pronounced when more $180^{\circ} \mathrm{rf}$-pulses are utilized. It can be explained by destructive coherence pathways that are created by imperfect rf-pulses as discussed in sec. 3.1.1.1. These destructive coherences are dephased by the gradient pulses once they are applied at sufficiently high gradient strengths. These points have been excluded from the fit. In sec. 4.2.2.2 it is attempted to recover these points using the PIETA approach.

\subsubsection{2d-MRPI calibration}

MRPI experiments may require utilization of the maximum gradient strength available with the gradient system. In this case, the high self-diffusion coefficient of $\mathrm{H}_{2} \mathrm{O}$ of sample CAL1 would lead to a too strong attenuation of the spin-echo signal. Thus, a substance with low self-diffusion coefficient was necessary to achieve sufficient signal-to-noise even for highest gradient strengths $G_{N}$. The sample CAL2 contains highly viscous PDMS and is therefore ideally suited. Figure 4.3 shows a surface plot of the magnitude of the spin echo maxima obtained when applying the 2d-MRPI pulse sequence to the sample CAL2. The parameters used were $N_{\mathrm{L}}=36, \delta^{\prime}=4.6 \mathrm{~ms}, \delta_{\mathrm{s}}=0.9 \mathrm{~ms}, G_{\mathrm{N}}^{\max }=1.45 \mathrm{Tm}^{-1}$ and $T_{\mathrm{K}}=293 \mathrm{~K}$. The observed spin-echo attenuation $E(\boldsymbol{q})$ exhibits rotational symmetry around the axis $\left(q_{x}, q_{y}\right)=(0,0)$ as expected for isotropic Gaussian self-diffusion. Figure 4.4 shows the spin-echo attenuation along the one-dimensional contour at $q_{\mathrm{x}}=0$. 


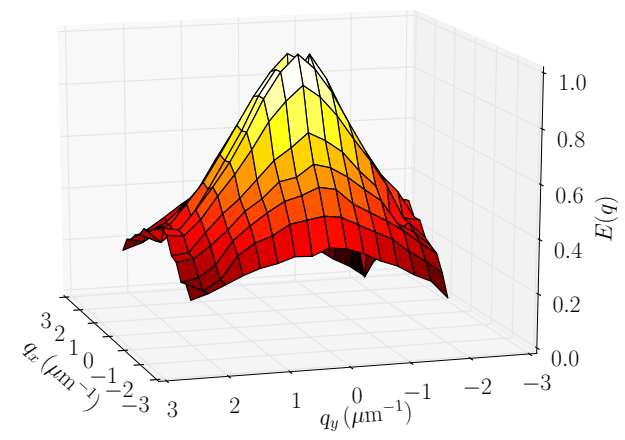

Figure 4.3: 2d-MRPI applied to PDMS. Shown is the spin echo maximum $E(\boldsymbol{q})$ obtained by sampling $\boldsymbol{q}$-space on a Cartesian grid.

The fit to eq. 4.2 yields the self-diffusion coefficient of $D=1.0 \pm 0.1 \times 10^{-12} \mathrm{~m}^{2} \mathrm{~s}^{-1}$ which lies in the expected range according to Appel and Fleischer (1993) and is consistent with a diffusion measurement utilizing a PGSE NMR stimulated echo pulse sequence. The first point in fig 4.4 is depressed due to destructive coherence pathways and was therefore disregarded in the analysis. Note that the second and third point may be attributed to a second faster diffusing component which may arise from molecules with shorter chain lengths. However, the second component can be attributed to PDMS molecules with comparable chain lengths as found in Appel and Fleischer (1993). In addition to

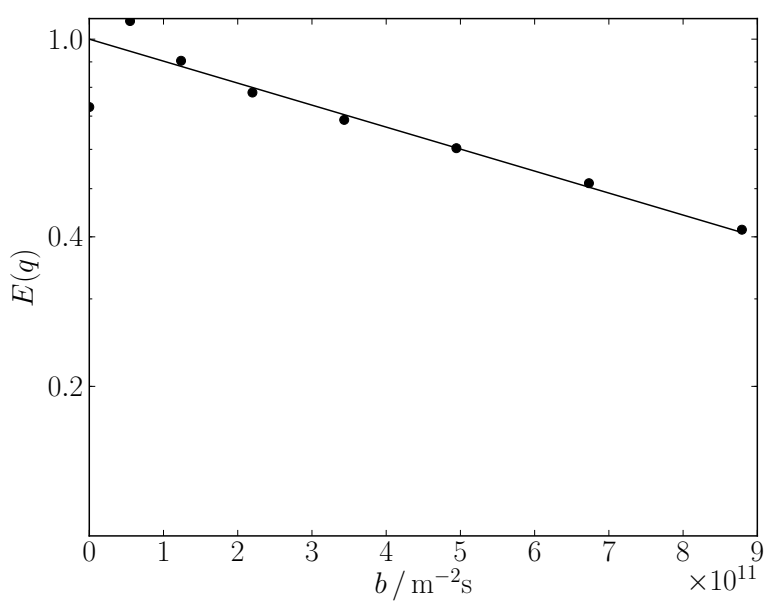

Figure 4.4: Spin-echo attenuation as obtained by extracting the one-dimensional contour at $q_{\mathrm{x}}=0$ from the data shown in fig. 4.3. The solid line shows the fit of the data to Eq. 4.2 disregarding the first three data points, which yields a self-diffusion coefficient of $D=1.0 \pm 0.1 \times 10^{-12} \mathrm{~m}^{2} \mathrm{~s}^{-1}$. 
the 2d-MRPI experiment, a 1d-MRPI experiment was performed as well to observe the spin-echo signal in the time domain up to the highest gradient strengths. The parameters used were $N_{\mathrm{L}}=50, \delta^{\prime}=4.1 \mathrm{~ms}, \delta_{\mathrm{s}}=0.9 \mathrm{~ms}, G_{\mathrm{N}}^{\max }=1.45 \mathrm{Tm}^{-1}$ and $T_{\mathrm{K}}=293 \mathrm{~K}$. Figure 4.5 shows the spin-echo signal in the time domain. The echo centers which are used to extract $E(q)$ are marked by red dots. The first spin-echo amplitude is depressed due to destructive coherence pathways as discussed in the previous section.

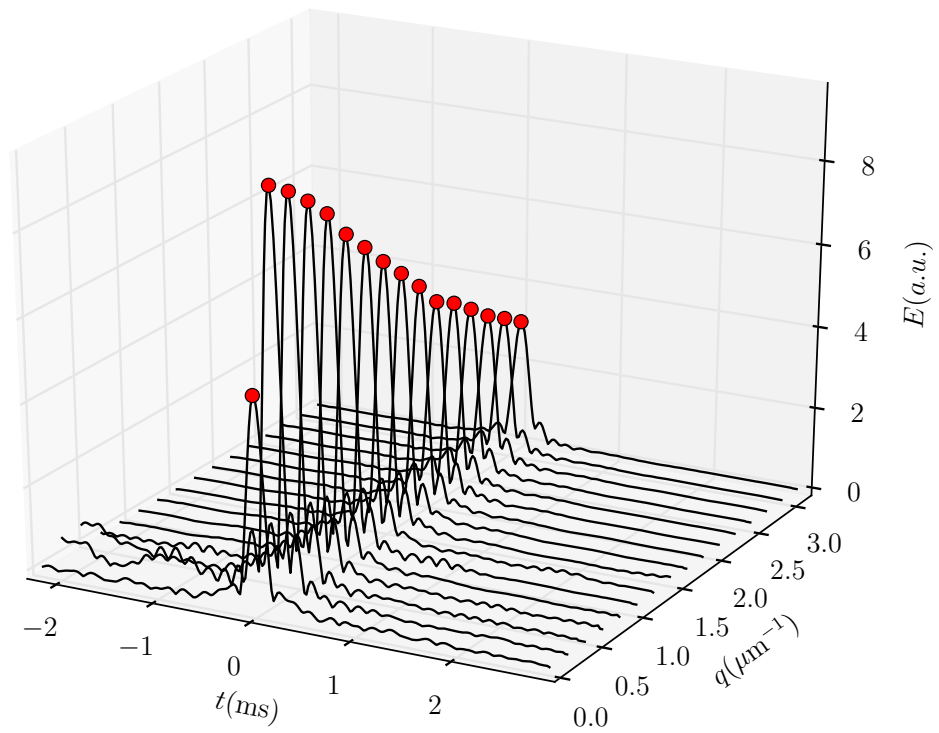

Figure 4.5: Spin-echo signal in the time domain as obtained by applying the 1d-MRPI pulse sequence to the sample CAL2. Echo centers are marked by red dots.

The spin-echo positions and spin-echo shapes in the time domain are stable for all gradient amplitudes as required for a well calibrated experimental setup. This way we ensured that there are no gradient mismatches and other spurious experimental artifacts.

\subsection{Cylindrical capillaries}

\subsubsection{Diffusive-diffraction PGSE NMR results}

Prior to the MRPI experiments it was checked whether the diffusive-diffraction PGSE NMR method can be successfully applied to the sample CYL1. Therefore, the PGSE 
NMR pulse sequence shown in fig. 2.9 was applied with the gradient direction perpendicular to the cylinder axis. For cylindrical pores the form factor expressed in terms of the wave vector $q$ is given by

$$
S_{0}(q)=\frac{J_{1}(2 \pi q L)}{\pi q L}
$$

where $J_{1}$ denotes the cylindrical Bessel function of first order. Three experiments were performed each with a gradient duration of $\delta=4 \mathrm{~ms}$ and the gradient amplitude was ramped from zero to a maximum value of $G_{\max }=1.16 \mathrm{Tm}^{-1}$ in 64 steps. Figure 4.6 shows the experimental data for three observation times $\Delta_{1}=20 \mathrm{~ms}$ (green dots), $\Delta_{2}=40 \mathrm{~ms}$ (yellow stars) and $\Delta_{3}=100 \mathrm{~ms}$ (red triangles). One may observe the convergence of the

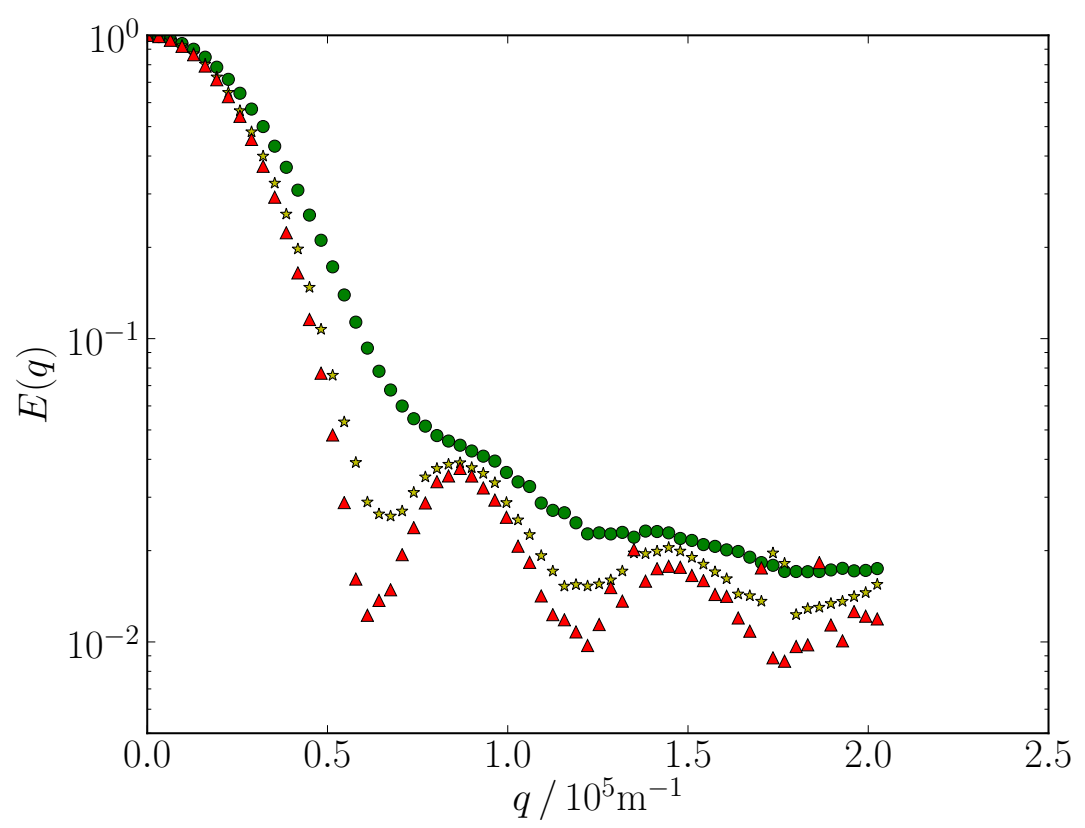

Figure 4.6: Diffusive-diffraction PGSE NMR data obtained by measuring restricted diffusion in the cylindrical capillaries of sample CYL1. Three observation times $\Delta$ were used, $\Delta_{1}=20 \mathrm{~ms}$ (green dots), $\Delta_{2}=40 \mathrm{~ms}$ (yellow stars) and $\Delta_{3}=100 \mathrm{~ms}$ (red triangles).

signal $E(q)$ to the average square of the form factor $\overline{\left|S_{0}(q)\right|^{2}}$ with increasing observation time $\Delta$. With a self-diffusion coefficient of $D=2.0 \times 10^{-9} \mathrm{~m}^{2} \mathrm{~s}^{-1}$ and a nominal radius of the capillaries of $L=10 \mu \mathrm{m}$, one obtains the ratios $D \Delta_{1} / L^{2}=0.4, D \Delta_{2} / L^{2}=0.8$ and $D \Delta_{3} / L^{2}=2.0$ consistent with the expected convergence according to Callaghan (1995). 
The pore density projected onto the radial direction $r$ for cylindrical pores is given by

$$
\rho_{0}(r)=2 \sqrt{L^{2}-r^{2}}
$$

As discussed in sec. 2.2.2, the diffusive-diffraction PGSE NMR technique shares the "phase problem" of diffraction techniques and therefore it only returns the pore density auto-correlation function $\rho_{0}^{*}(R)$ upon Fourier transform of the $\boldsymbol{q}$-space data. $\rho_{0}^{*}(R)$ is plotted in fig. 4.7 (black) for the cylinders used in the experiments above. It was obtained by inserting eq. 4.4 into eq. 1.11 and numerically evaluating the integral. The Fourier

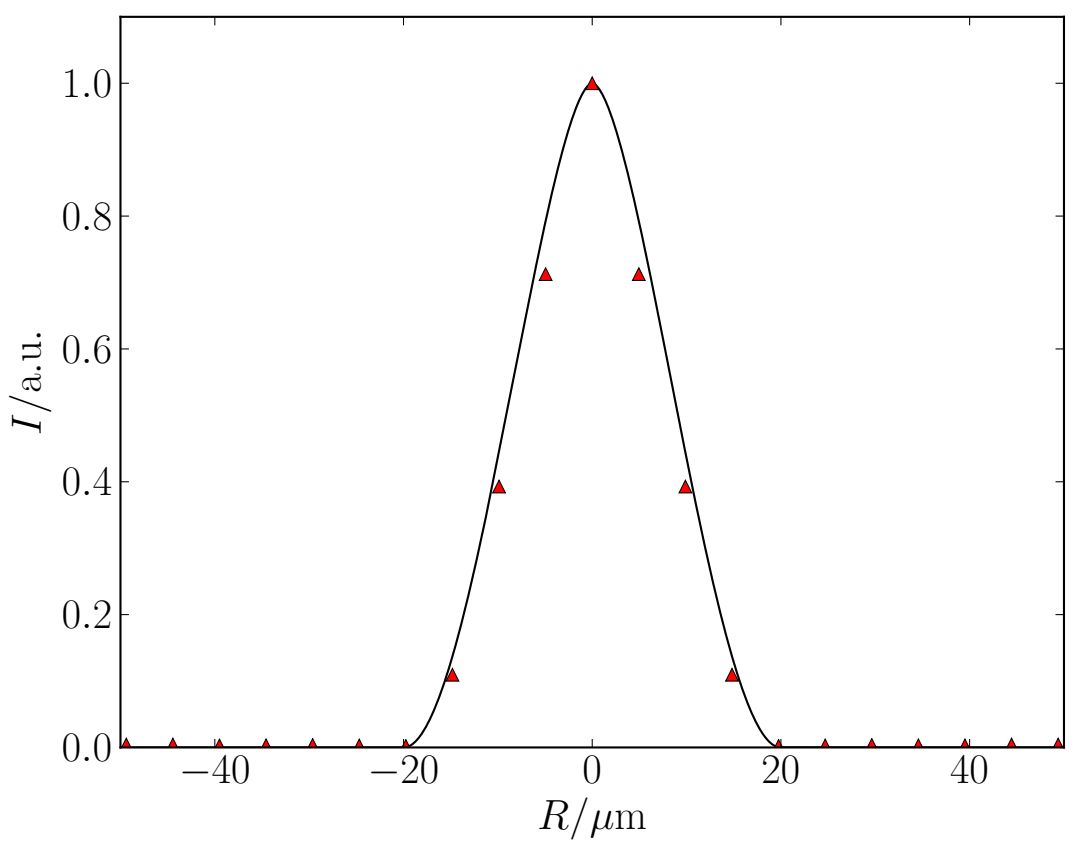

Figure 4.7: Pore density auto-correlation function $\rho_{0}^{*}(R)$ for the cylindrical domain with radius $L=10 \mu \mathrm{m}$ (black). Fourier transform of the diffusive-diffraction PGSE NMR data with the observation time $\Delta_{3}=100 \mathrm{~ms}$ (red triangles).

transform of the experimental data for the observation time $\Delta_{3}=100 \mathrm{~ms}$ is shown in fig. 4.7 (red triangles). One may notice the extension of $\rho_{0}^{*}(R)$ from $-2 L=-20 \mu \mathrm{m}$ to $2 L=20 \mu \mathrm{m}$. Additionally, one may compare the pore auto-correlation function as shown in fig. 4.7 and for the parallel plane as plotted in fig. 1.3. A comparison between the two domains shows that the underlying pore density is obscured which prevents direct conclusions about the pore shape just by analyzing the Fourier transformed data. 
Moreover, the experimental data and theory agree within uncertainty, thus proving a well adjusted experimental setup and showing that the sample CYL1 is well suited for the following MRPI experiments.

\subsubsection{1d-MRPI results}

After successful calibration, we applied the 1d-MRPI pulse sequence as shown in fig. 3.2 to the sample CYL1 perpendicular to the capillary axis. The capillaries were aligned with the main magnetic field $B_{0}$. The following set of parameters was used $N_{\mathrm{L}}=36$, $\delta^{\prime}=3.045 \mathrm{~ms}$ (accounts for the ramping time), $2 \tau=5.56 \mathrm{~ms}$ and the read gradient was applied for $\delta_{\text {read }}=2.12 \mathrm{~ms}$ with $2 \%$ of the maximum gradient amplitude. The two narrow gradients $G_{\mathrm{N}}$ were ramped up to a maximum of $G_{\max }=1.45 \mathrm{Tm}^{-1}$ and a total of 64 gradient steps were utilized for the MRPI gradient scheme.

Multiplying the number of long gradient intervals with their time separation $2 \tau$ yields an approximate time of the gradient pulse scheme of $T=200 \mathrm{~ms}$. This time is an important parameter to estimate whether the condition $D T / L^{2} \gg 1$ is fulfilled. At a sample temperature of $T_{\mathrm{K}}=293 \mathrm{~K}$ one obtains a ratio of $D T / L^{2}=4$. As discussed in sec. 2.2.3, a higher ratio $D T / L^{2}$ may be advantageous to observe the average form factor. Thus, for this proof of concept experiment we chose to increase this ratio by heating the sample to a temperature of $T_{\mathrm{K}}=313 \mathrm{~K}$, which increases the self-diffusion coefficient to $D=3.2 \times 10^{-9} \mathrm{~m}^{2} \mathrm{~s}^{-1}$ according to Holz et al. (2000). Thus, for the experiment presented in this section the ratio is given by

$$
\frac{D T}{L^{2}}=6.4
$$

Figure 4.8 shows the real $(+)$ and imaginary part $(\times)$ of the MRPI spin-echo amplitude $E(\boldsymbol{q})$ versus the applied $q$-values. The solid line in fig. 4.8 shows the simulation based on the MCF technique, when setting the radius to a value of $L=10 \mu \mathrm{m}$. The agreement between experiment and simulation is remarkable. These experimental results proved for the first time that the complete form factor for microscopic pores can be measured using the MRPI approach (Hertel et al. (2013)). Most notably, the real part in fig. 4.8 


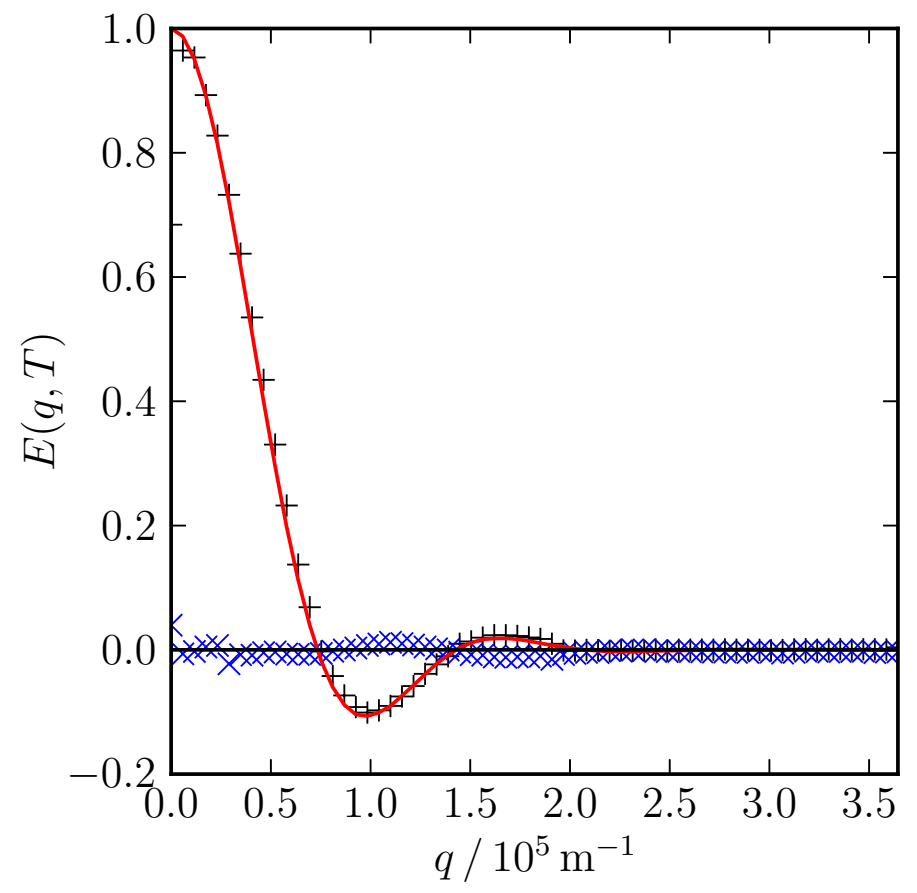

Figure 4.8: Real $(+)$ and imaginary $(\times)$ part of the spin-echo amplitude $E(\boldsymbol{q})$. The solid line was obtained by MCF simulation. (Figure from S. Hertel, M.Hunter and P. Galvosas, Phys. Rev. E, 87, 030802(R), 2013 "Copyright 2013 by the American Physical Society.").

exhibits both positive and negative values, while the imaginary part is close to zero as required for a sample with cylindrical symmetry. Thus, the "phase problem", which is limiting the information obtainable from diffraction techniques in general is lifted using MRPI. Moreover, comparing fig. 4.8 and the results obtained using diffusive-diffraction PGSE NMR (see fig. 4.6), one may observe a much higher signal-to-noise for the MRPI experiment. This is a consequence of measuring $\overline{S_{0}(q)}$ in MRPI as compared to $\overline{\left|S_{0}(q)\right|^{2}}$ in diffusive-diffraction PGSE NMR. Note that the first three data points in fig. 4.8 are depressed similar to the observations made in the calibration experiments in sec. 4.1.2. However, the amplitudes of these data points may be recovered using the PIETA approach as will be shown in sec. 4.2.2.2.

One may obtain an apparent radius $L_{\text {app }}$ of the pores by comparing the measured data shown in fig. 4.8 to the form factor given by eq. 4.3. For this purpose, one may read off the zero crossings of the real part of the signal $E(q)$ and relate the $q$-value to the radius using the relation $\alpha_{\mathrm{i}}=2 \pi q L$, where $\alpha_{\mathrm{i}}$ is the $i$-th zero of the cylindrical 
Table 4.1: The first three zeros of $J_{1}(x)$ and the zeros of the NMR signal $E(q)$ obtained from the MRPI and diffusive-diffraction PGSE-NMR data together with the derived apparent capillary radius $L$.

\begin{tabular}{lccccc}
\hline \hline & Zeros of $J_{1}(x)$ & \multicolumn{2}{c}{ MRPI } & \multicolumn{2}{c}{ PGSE-NMR } \\
& $x=2 \pi L q$ & $q\left(\mu m^{-1}\right)$ & $L_{\text {app }}(\mu m)$ & $q\left(\mu m^{-1}\right)$ & $L_{\text {app }}(\mu m)$ \\
\hline First & 3.8317 & 0.075 & 8.1 & 0.066 & 9.2 \\
Second & 7.0156 & 0.146 & 7.7 & 0.117 & 9.5 \\
Third & 10.1735 & 0.197 & 8.2 & 0.170 & 9.5 \\
\hline \hline
\end{tabular}

Bessel function $J_{1}$. Table 4.1 shows a comparison of the first three zeros for the MRPI data shown in fig. 4.8 and the diffusive-diffraction PGSE NMR data shown in fig. 4.6. The zeros of the MRPI data are shifted towards higher $\boldsymbol{q}$ values compared to the zeros of the diffusive diffraction PGSE-NMR experiments. Thus, the pores appear smaller in the MRPI experiments than the actual radius $L=10 \mu \mathrm{m}$ of the capillaries. This difference may be explained by the edge enhancement effect discussed in sec. 2.2.3.3. Edge enhancement is caused by the shift of the center of masses of Brownian motions away from the capillary boundary. This leads to an apparent shrinkage of the pores, which scales with the duration of the refocusing gradient pulse. The diffusive-diffraction PGSE NMR experiments were carried out with narrower gradient pulses of $\delta=4 \mathrm{~ms}$. This compares to an effective duration of the narrow gradient consisting of two gradient pulses in the MRPI experiment of $\delta_{N, \text { eff }}=8.1 \mathrm{~ms}$. The narrower pulse in the diffusivediffraction PGSE NMR experiments allows the centre of mass to be closer to the pore wall which consistently yields a larger radius (last column in tab. 4.1).

Further, evidence of this effect can be obtained by performing the Fourier transform of the experimental MRPI data. Figure 4.9 (red) shows the real part of the Fourier transform of the MRPI $\boldsymbol{q}$-space data. For comparison the pore density projection of a cylindrical pore which is given by eq. 4.4 is plotted in fig. 4.9 (black). Figure 4.9 shows that the pore density projection can be measured with MRPI, while for conventional diffusive-diffraction PGSE NMR the observed function returns the much broader pore density correlation function as shown in fig. 4.7. One may observe that the measured pore density projected onto the gradient direction (fig. 4.9 (red)) appears narrower than the theoretical pore density $\rho_{0}(r)$ given by eq. 4.4 and shown in fig. 4.9 (black). This 


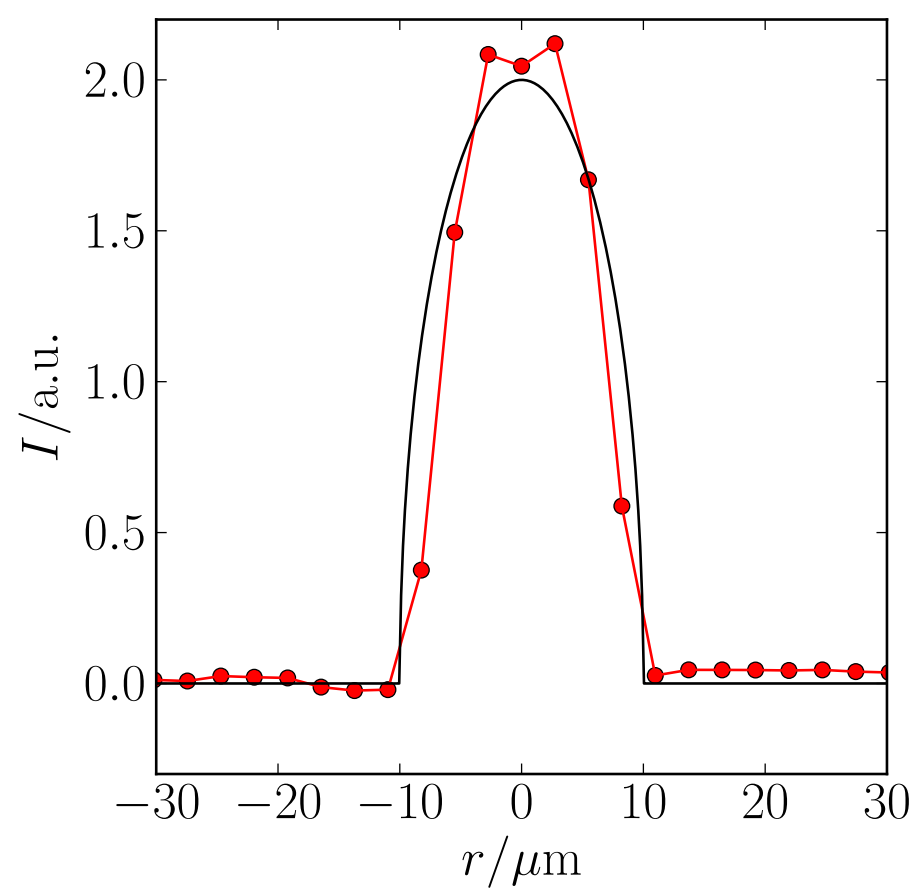

Figure 4.9: $1 \mathrm{~d}$ projection of the pore density as obtained by Fourier transform of the q-space data shown in fig. 4.8 (red). Pore density of a cylindrical pore according to eq. 4.4 (black).

observation is consistent with the analysis of the apparent radius $L_{\text {app }}$ using the zero crossings shown in tab. 4.1. Furthermore, there are two points with enhanced signal amplitude on the top of the experimental pore density, which is due to the effect of edge enhancement (sec. 2.2.3.3). However, the correct pore dimensions can be extracted when taking into account these effects as will be discussed in the following sections.

\subsubsection{MRPI convergence to the form factor}

It was already noted with the first theoretical simulations by Laun et al. (2012) that the MRPI pulse sequence may need considerably more time for convergence than diffusivediffractive PGSE NMR. However, it was not yet clear which ratios $D T / L^{2}$ would be needed to allow structure determination. Figure 4.10 shows the convergence of the signal $E(q)$ to the form factor $S_{0}(q)$ for a series of experiments with increasing ratio $D T / L^{2}$. 


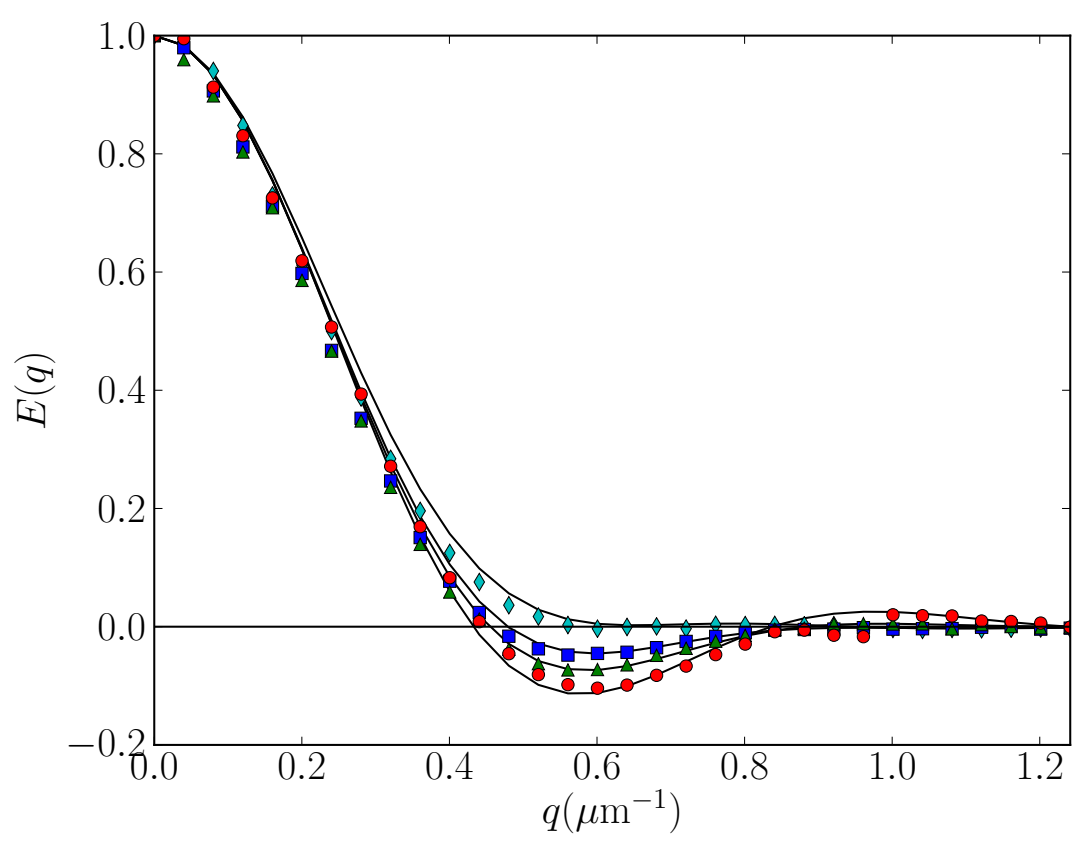

Figure 4.10: Real part of the normalized spin-echo amplitude $E(q)$ versus wave vector $q$ for increasing ratio $D T / L^{2}$. The total time of the gradient pattern $T$ of the MRPI experiment was increased by increasing the number of long gradient pulses $N_{\mathrm{L}}$ and keeping all other parameters fixed. The shown experimental data corresponds to $D T / L^{2}=1.1$ (diamonds), $D T / L^{2}=2.1$ (squares), $D T / L^{2}=3.0$ (triangles) and $D T / L^{2}=5.8$ (circles). MCF simulation for each experiment is shown as black lines.

The total time of the gradient pattern $T$ of the MRPI experiment was increased by increasing the number of long gradient pulses $N_{\mathrm{L}}$ while keeping all other parameters fixed. The MRPI parameters were $2 \tau=4.725 \mathrm{~ms}, \delta^{\prime}=4 \mathrm{~ms}$ and $G_{\max }=0.58 \mathrm{Tm}^{-1}$. In contrast to the experiments shown in sec. 4.2.2, here the sample was not heated, but was kept at a temperature of $T_{\mathrm{K}}=293 \mathrm{~K}$ leading to a self-diffusion coefficient of $D=2.0 \times 10^{-9} \mathrm{~m}^{2} \mathrm{~s}^{-1}$. The number of long gradient pulses was varied and the values $N_{\mathrm{L}}=\{10,20,30,50\}$ were applied, leading to ratios of $D T / L^{2}=\{1.1,2.1,3.0,5.8\}$. In fig. 4.10 the spin-echo amplitude $E(q)$ contains the first negative lobe of the form factor even for moderate durations $T$ of the MRPI gradient pattern. At room temperature the parameters $N_{\mathrm{L}}=20$ and thus $T=103 \mathrm{~ms}$ were sufficient to extract useful information about the pore structure. Observation of the second lobe and therefore more detailed information about the structure requires more long gradient pulses e.g. $N_{\mathrm{L}}=50$ and thus $D T / L^{2}=5.8$ for the parameters used in this work. 


\subsubsection{MRPI - PIETA phase cycle}

As discussed in sec. 3.1.1.1 at low gradient strengths additional coherence pathways may influence the signal. In order to recover the correct signal intensity the PIETA rf-pulse phase scheme discussed in sec. 2.3.4 was applied to the 1d-MRPI rf-pulse scheme. For this purpose the rf-pulse phases $\Phi_{2}$ and $\Phi_{4}$ (as assigned in fig. 3.1.1) were incremented from 0 to $2 \pi$. The number of long gradient pulses was set to $N_{\mathrm{L}}=30$ and thus the measured spin echo corresponds to the spin echo number $n=32$. According to eq. 2.102 the number of phase steps was set to $N_{\text {scan }}=2 n=64$ and the direct coherence pathway was expected at $\Delta P=32$ as discussed in sec. 2.3.4. The other rf-pulse phases were kept constant at $\Phi_{1}=\Phi_{\text {rec }}=0$ and $\Phi_{3}=\pi / 2$, respectively. The resulting data set had dimensions of $512 \times 16 \times 64$, where 512 points were acquired in the time domain, $\boldsymbol{q}$ was incremented in 16 steps and 64 phase steps were acquired. A subsequent Fourier transform in the phase direction allowed to extract the direct coherence pathway.

Figure 4.11 shows the total change in coherence $\Delta P$ for each step in $q$. The form factor can be obtained by selecting the direct pathway with $\Delta P=32$. Furthermore,

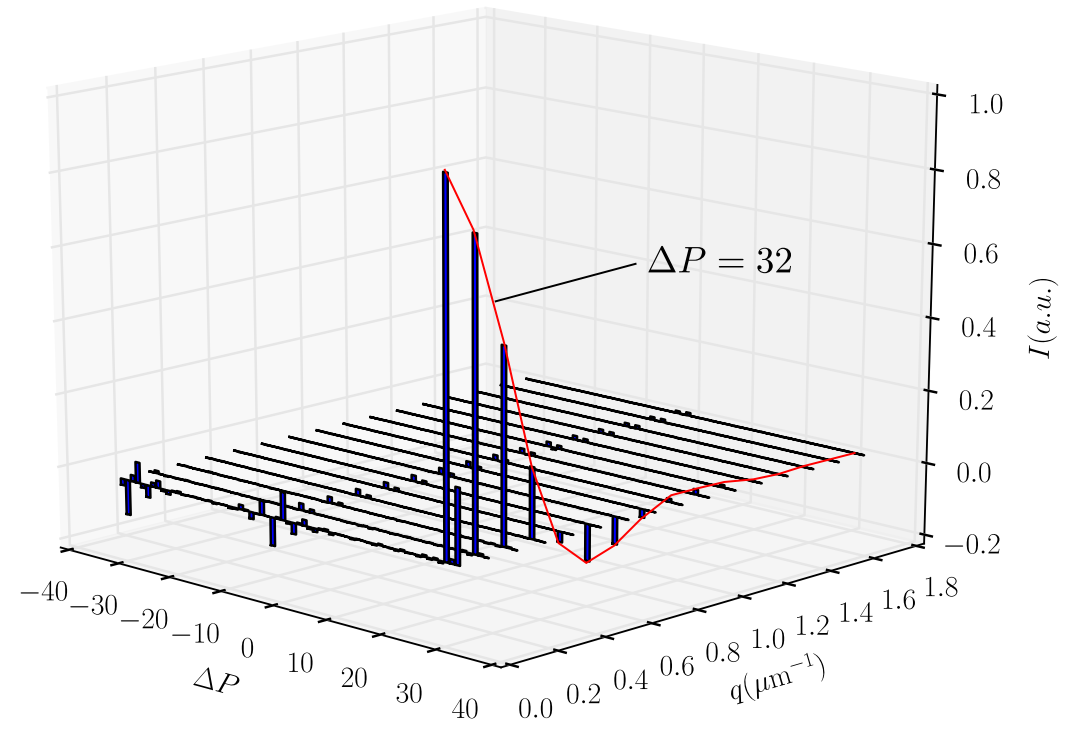

Figure 4.11: Coherence transfer pathway map obtained by applying MRPI including the PIETA phase cycling scheme to the sample CYL1. Real part of the coherence pathways with total coherence change $\Delta P$ for the center of the spin echo. The desired pathway is the direct coherence pathway with $\Delta P=32$. 
one may identify destructive coherence pathways with enough intensity to significantly impact on the spin echo amplitude. Such pathways are the ones close to $\Delta P=0$, which are stimulated echo pathways with many intervals where the magnetization is stored in the $z$-direction $\left(p_{\mathrm{k}}=0\right)$. Secondly, coherence pathways close to the direct pathway with $\Delta P=32$ and $\Delta P=-32$ also appear with significant intensity. These pathways are characterized by a small number of time intervals where the magnetization is stored in $z$-direction. Note that with higher gradient strength and thus higher $q$-values, the destructive coherence pathways are dephased and only the direct pathway contains significant intensity.

Figure 4.12 (b) shows the complex form factor as obtained by selecting the spinecho center of the direct coherence pathway for each step in $\boldsymbol{q}$-space. As predicted, the points for low gradient strength were recovered. Moreover, when comparing the signal amplitude for low $\boldsymbol{q}$ values between fig. 4.12 (a) and (b) one may notice that the imaginary part (squares) is closer to zero in fig. 4.12 (b) as required by the cylindrical pore shape.
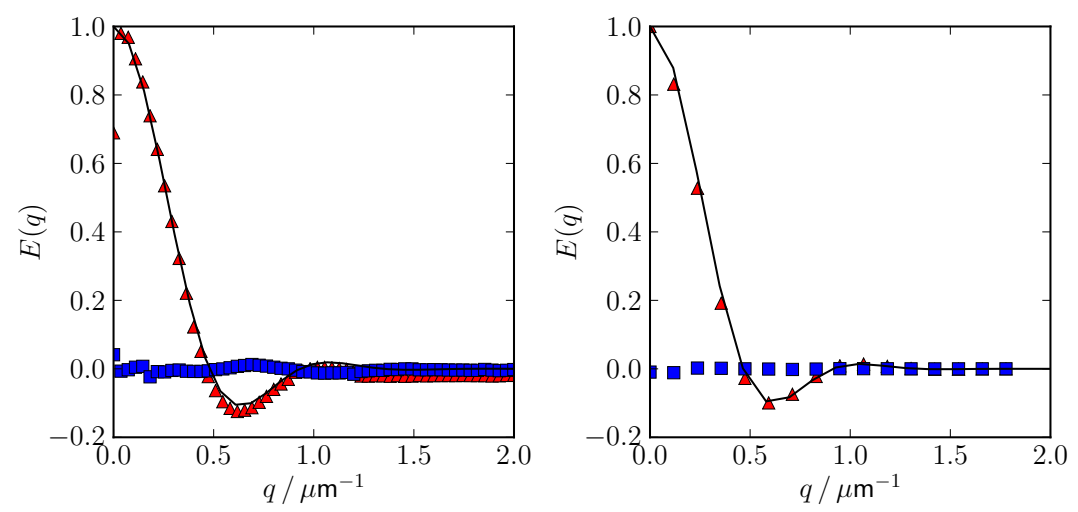

Figure 4.12: Real (triangles) and imaginary part (squares) of the $\boldsymbol{q}$-space profile of sample CYL1 as obtained by the 1d-MRPI pulse sequence. This figure is identical to fig. 4.8 , which is reprinted here for better comparison. The spin echo amplitude is distorted at low gradient strengths due to destructive coherence pathways (a). The PIETA rf-pulse phase scheme allows to extract the direct pathway and to recover the points at low gradient strengths (b). The solid line was obtained by MCF simulation (a) and (b).

Note that the experiment time increases by a factor of 16 when comparing with the 4 step phase cycle. Therefore, this phase scheme is not feasible for multi-dimensional 
MRPI applications. However, by analyzing the coherence pathway spectrum contributing to the echos, one is in principle able to identify the most important spurious coherence pathways. Based on this information one can design shorter phase schemes to eliminate them, depending on the particular conditions of the experiment (Hertel and Galvosas $(2015))$.

\subsubsection{2d-MRPI results}

While 1d-profiles of the pores may be suited to determine the characteristic length of the underlying pore structure, it may be desired to obtain the actual pore shape. The pore shape as well as its size may be directly obtained by applying the 2d-MRPI approach as discussed in sec. 3.1.2.1. Thus, the $2 \mathrm{~d}$-approach may be considered as generally superior to the 1d-MRPI experiment, however, the additional information gained comes at the expense of experimental time.

We have applied a two-dimensional MRPI experiment following the 2d-Fourier transform approach discussed in sec. 3.1.2.1 to the sample CYL1. The parameters of the MRPI gradient pattern were $N_{\mathrm{L}}=36, \delta^{\prime}=3.045 \mathrm{~ms}$ (includes the ramping time), $2 \tau=5.56 \mathrm{~ms}$ and the sample was heated to a temperature of $T_{\mathrm{K}}=313 \mathrm{~K}$. The gradients were stepped independently to traverse $\boldsymbol{q}$-space in a $32 \times 32$ matrix as discussed in sec. 3.1.2.1.

The result as shown in fig. 4.13 (a) is the real part of the two-dimensional form factor which inherits its point symmetry from the object it is describing - the water confined by the capillary walls. 

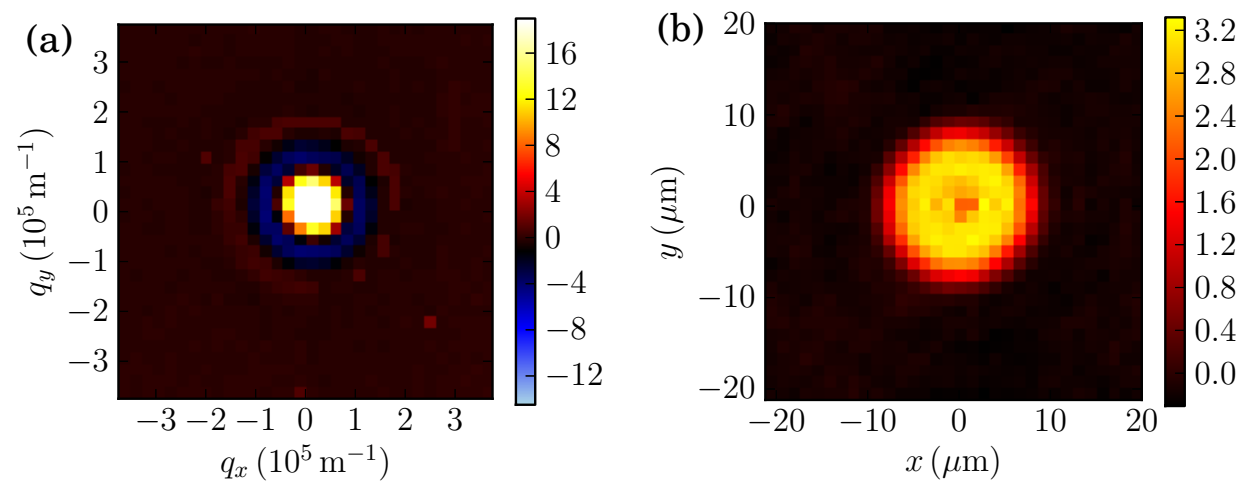

Figure 4.13: Real part of the two-dimensional form factor as acquired with MRPI from water in a bundle of capillaries (a) and the corresponding averaged pore image obtained by a subsequent $2 \mathrm{~d}$ Fourier transform of these data (b). (Figures from S. Hertel, M.Hunter and P. Galvosas, Phys. Rev. E, 87, 030802(R), 2013 "Copyright 2013 by the American Physical Society.")

As for the one-dimensional form factor shown in fig. 4.8, the imaginary part of the acquired signal (not shown) is close to zero in the two-dimensional data set. It is most intriguing to see in fig. 4.13 (b) that a simple two-dimensional Fourier transform reconstructs the actual pore shape, averaged over all pores. Remarkably, the nominal resolution of this image is $1.3 \mu \mathrm{m} /$ pixel, however it was achieved with moderate gradients provided by a standard micro-imaging system.

Figure 4.14 (dots) shows the cross section through the pore image shown in fig. 4.13 (b) at $y=0$. The solid line in fig. 4.14 shows the data as obtained by MCF simulation. Simulation and experiment agree remarkably well, since the MCF simulation was carried out using the CPMG based gradient scheme of the MRPI pulse sequence shown in fig. 3.2. Thus, the effects of blurring (sec. 2.2.3.2) and edge enhancement (sec. 2.2.3.3) are accounted for. One may estimate the peak of the edge enhancement with eq. 2.65 assuming a locally flat surface. With an effective duration of the narrow gradient of $2 \delta^{\prime}=6.09 \mathrm{~ms}$ this peak is expected at $x_{\max }=3.1 \mu \mathrm{m}$. However, comparison with fig. 4.14 shows that the peaks are shifted further towards the center of the pore due to the finite duration of the long gradient as discussed in sec. 2.2.3.3.

Note that the pore density obtained by extracting a cross section through the $2 \mathrm{~d}$-pore image is different from the pore density projection obtained by the 1d-MRPI experiment shown in fig. 4.9. Using the $2 \mathrm{~d}$-approach one may extract the one-dimensional pore 


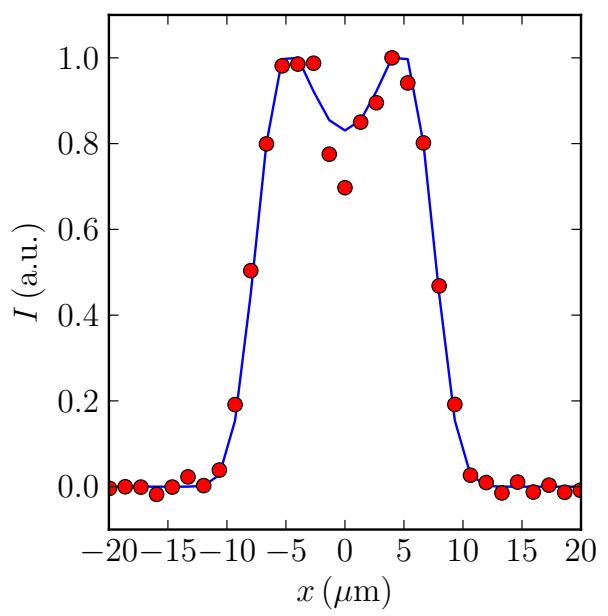

Figure 4.14: One-dimensional cross section through the 2d-MRPI image at $y=0$ (dots) and MCF simulation (solid line). The combined effect of blurring and edge-enhancement for the given experimental parameters are captured by the MCF simulation.

density, which resembles the local spin density and can be described by the convolution of eq. 2.61 and eq. 2.64. In contrast, the 1d-MRPI approach measures the pore spin density projected onto the gradient direction, which is given by eq. 4.4. Furthermore, the resolution in fig. 4.14 is twice that of fig. 4.9 due to the use of bipolar gradients which allows to scan $\boldsymbol{q}$-space symmetrically in the 2d-MRPI experiment, while only one side of $\boldsymbol{q}$-space was sampled in the 1d-MRPI experiments.

\subsection{Triangular capillaries}

MRPI experiments on the hemi-equilateral triangular capillary sample TRI pose several additional challenges. The solid pore matrix consists of amorphous poly-carbonate, which is partially permeable for $\mathrm{H}_{2} \mathrm{O}$ molecules. Thus, there is exchange of molecules between the capillary space and the solid matrix during the MRPI experiment. Moreover, there is a certain amount of surface relaxation at the polycarbonate- $\mathrm{H}_{2} \mathrm{O}$ interface. Both processes have similar effects on the MRPI signal and can be regarded as a finite absorption probability of the diffusing $\mathrm{H}_{2} \mathrm{O}$ molecules at capillary boundaries. The experimental determination of the rate constants of both processes is possible, but time consuming and might warrant a study of its own. 
However, the presence of surface absorption has two additional consequences for the MRPI experiments. First, it impacts on the transverse relaxation time constant $T_{2}$, which will be shortened as compared to bulk $\mathrm{H}_{2} \mathrm{O}$. Thus, the time available for the application of the long gradient $G_{\mathrm{L}}$ will be shortened. Utilizing the CPMG rf-pulse sequence discussed in sec. 2.1.4 this time constant was determined to be $T_{2}=600 \mathrm{~ms}$. Second, the water molecules may leave the pore space much more efficiently than in the glass capillaries utilized in the previous sections. Thus, over the time it takes to acquire a 2d-MRPI data set on a Cartesian grid (Fourier transform approach discussed in sec. 3.1.2.1.) the triangular capillaries may not be homogeneously filled by $\mathrm{H}_{2} \mathrm{O}$ anymore. Therefore, the Radon transform approach as discussed in sec. 3.1.2.2 provides an advantage over the 2d-Fourier transform approach. By allowing to acquire a set of 1d $\boldsymbol{q}$-space profiles, the acquisition may be interrupted to check the sample condition by acquiring MRI images. Furthermore, individual $\boldsymbol{q}$-space profiles may be compared to simulations and can be repeated if any inconsistency was detected. The following section will discuss MCF simulations of the $2 \mathrm{~d}$ average pore images obtained using the Radon transform approach.

\subsubsection{Multiple Correlation Function simulations}

MCF simulations allow to efficiently study the influence of the parameters of the MRPI pulse sequence such as the effective durations of the long and short gradient pulses on the acquired pore images. The work presented in this thesis shows the first experiments of MRPI utilizing microscopic triangular pores. To our knowledge, no prior experimental experience existed about the optimal range of parameters and the expected quality of the average pore images. Therefore, the following simulations were carried out before performing the experiments to find a set of optimal parameters for the MRPI pulse sequence given experimental constrains such as $T_{2}=600 \mathrm{~ms}$. Further experimental constraints were the self-diffusion coefficient of $\mathrm{H}_{2} \mathrm{O}$ at a temperature of $T_{\mathrm{K}}=293 \mathrm{~K}$ of $D=2.0 \times 10^{-9} \mathrm{~m}^{2} \mathrm{~s}^{-1}$, the maximal gradient strength of $G_{\max }=1.45 \mathrm{Tm}^{-1}$ and the minimal separation time between gradient pulses and rf-pulses of $0.25 \mathrm{~ms}$. Note the necessity for the minimum delay of $0.25 \mathrm{~ms}$ was discussed in sec. 3.2.3. 
First, the influence of the effective duration of the narrow gradient pulse $G_{\mathrm{N}}$ was investigated. Other parameters were set to similar values as utilized for the sample CYL1, as described in the previous sections. The initial parameters were $2 \tau=6 \mathrm{~ms}, \delta^{\prime}=5.5 \mathrm{~ms}$, $N_{\mathrm{L}}=36$ and the number of angular $\boldsymbol{q}$-space profiles (discussed in sec. 3.1.2.2) was $N_{\theta}=18$. Figure 4.15 shows the obtained pore images for the the number of narrow gradients $N_{\mathrm{N}}=2(\mathrm{a}), N_{\mathrm{N}}=4(\mathrm{~b}), N_{\mathrm{N}}=6$ (c), $N_{\mathrm{N}}=8$ (d). With increasing effective
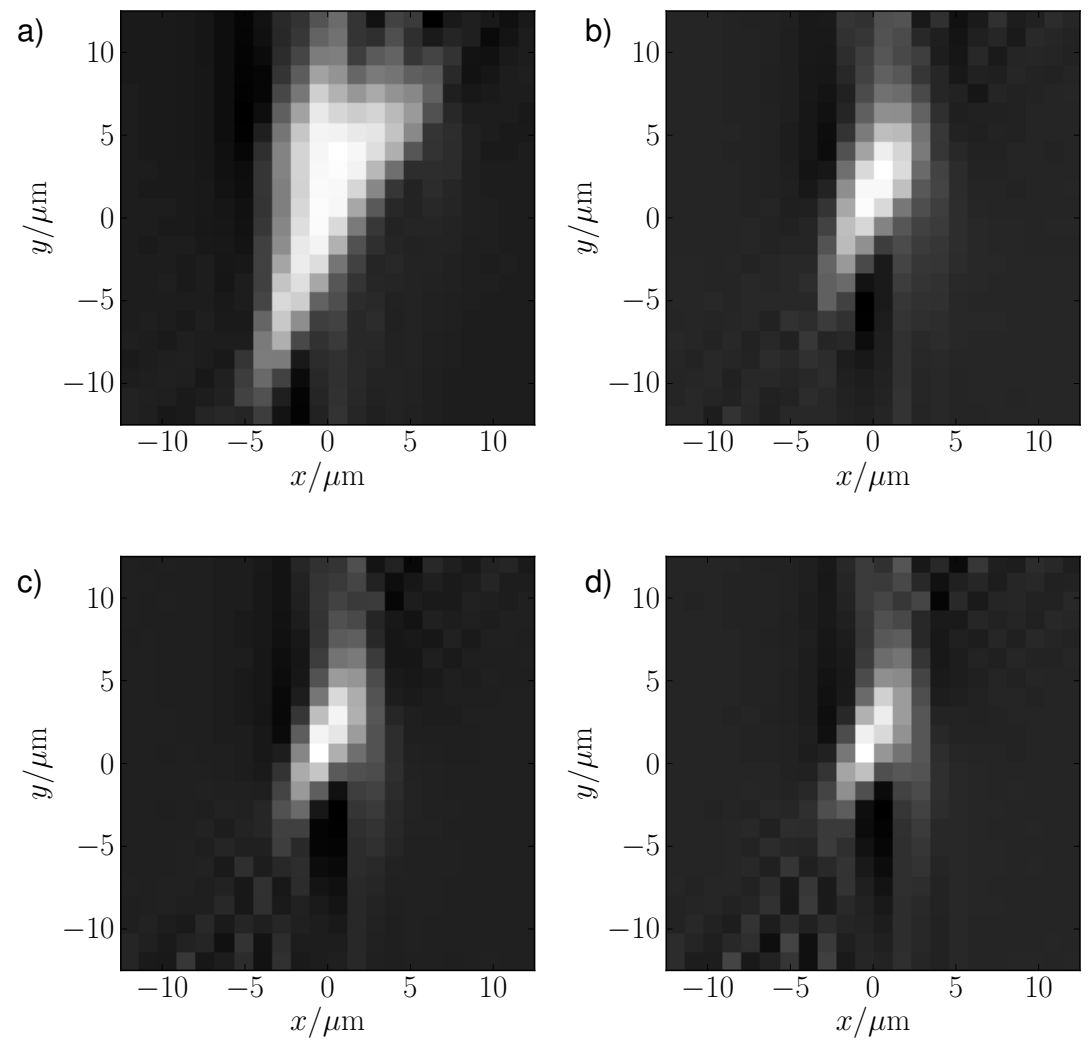

Figure 4.15: $\mathrm{MCF}$ simulated pore images of the hemi-equilateral triangular domain. Number of narrow gradients was varied to $N_{\mathrm{N}}=2$ (a), $N_{\mathrm{N}}=4$ (b), $N_{\mathrm{N}}=6$ (c), $N_{\mathrm{N}}=8$ (d).

duration of the narrow gradient pulse the edge enhancement effect is expected to shift intensity towards the center of the pore space. As can be seen this effect is severe for the images fig. 4.15 b), c) and d) and the pore shape is lost. In contrast, the image fig. 4.15 a) shows the hemi-equilateral triangular shape.

Subsequently, the influence of the effective duration of the long gradient $G_{\mathrm{L}}$ was studied by varying the number of long gradient segments $N_{\mathrm{L}}$ while keeping all other parameters fixed. Especially, the number of narrow gradient segments was kept at 
$N_{\mathrm{N}}=2$. Figure 4.16 shows the obtained pore images for the the number of long gradients $N_{\mathrm{L}}=36(\mathrm{a}), N_{\mathrm{L}}=26(\mathrm{~b}), N_{\mathrm{L}}=16(\mathrm{c})$ and $N_{\mathrm{L}}=6(\mathrm{~d})$.
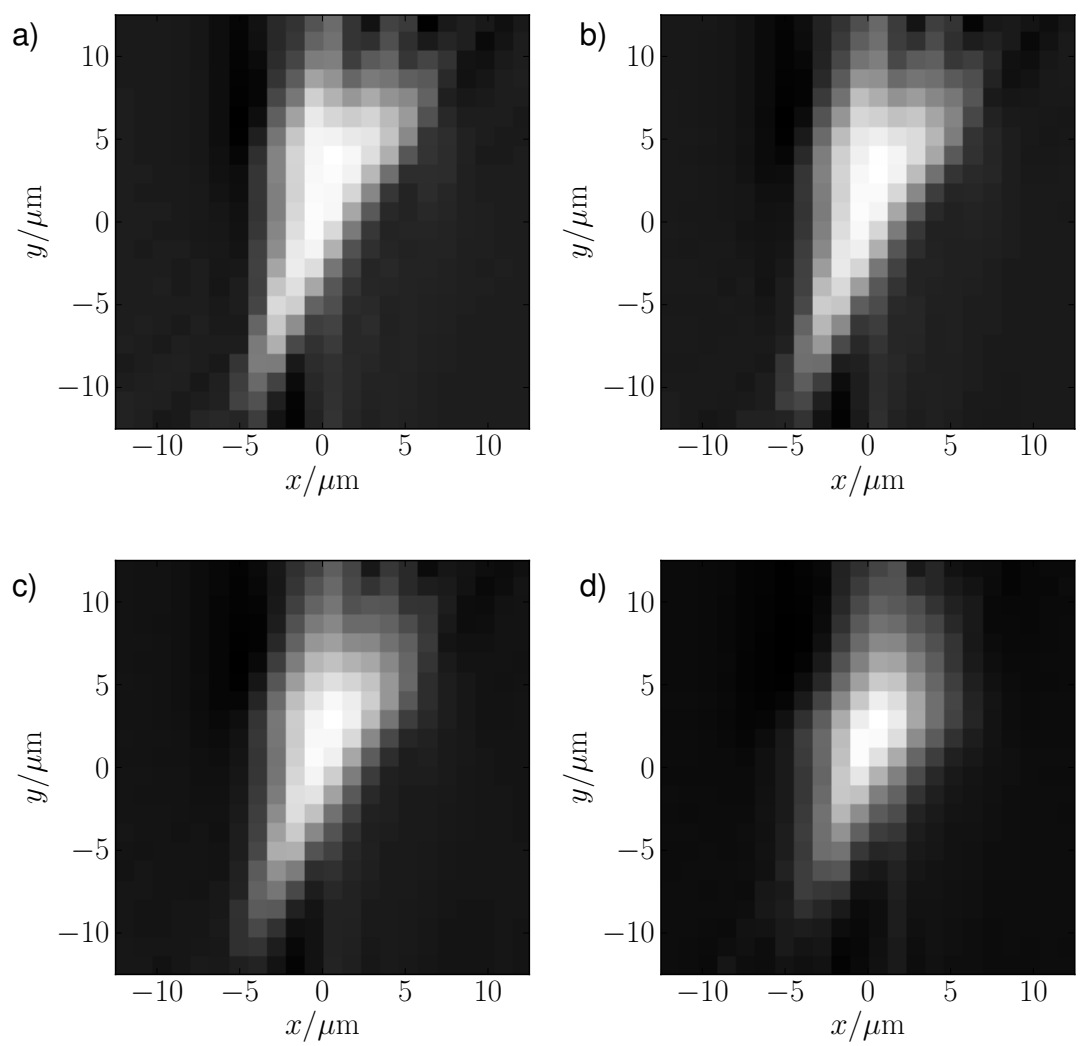

Figure 4.16: $\mathrm{MCF}$ simulated pore images of the hemi-equilateral triangular domain. Number of long gradients $N_{\mathrm{L}}=36(\mathrm{a}), N_{\mathrm{L}}=26(\mathrm{~b}), N_{\mathrm{L}}=16(\mathrm{c})$ and $N_{\mathrm{L}}=6(\mathrm{~d})$.

Figure 4.16 (a) shows the sharpest hemi-equilateral pore shape due to the largest effective duration of the long gradient. With decreasing effective duration of the long gradient pulse the pore images get blurred. Especially, in fig. 4.16 (c) and (d) the effective duration is too short and leads to severe burring. However, fig. 4.16 (b) with $N_{\mathrm{L}}=26$ still shows the triangular shape with well defined edges. In experiments less rf-pulses and a shorter effective duration is desirable due to decreasing experimental artifacts. Thus, the number of long gradient segments was set to $N_{\mathrm{L}}=26$ for the experiments discussed in the following sections.

Utilizing the optimized parameters found by the simulations above, the influence of the number of radial $\boldsymbol{q}$-space profiles $N_{\theta}$ was studied. The aim was to identify the least number of profiles, since experiment time is directly proportional to this number. All 
other parameters were fixed and were $2 \tau=6 \mathrm{~ms}, \delta^{\prime}=5.5 \mathrm{~ms}, G_{\max }=1.45 \mathrm{Tm}^{-1}$. The number of short gradient segments was set to $N_{\mathrm{N}}=2$ and the number of long gradient segments was set to $N_{\mathrm{L}}=26$. Figure 4.17 shows the obtained pore images for the number of radial profiles $N_{\theta}=9$ (a), $N_{\theta}=18$ (b), $N_{\theta}=36$ (c), $N_{\theta}=54$ (d). With
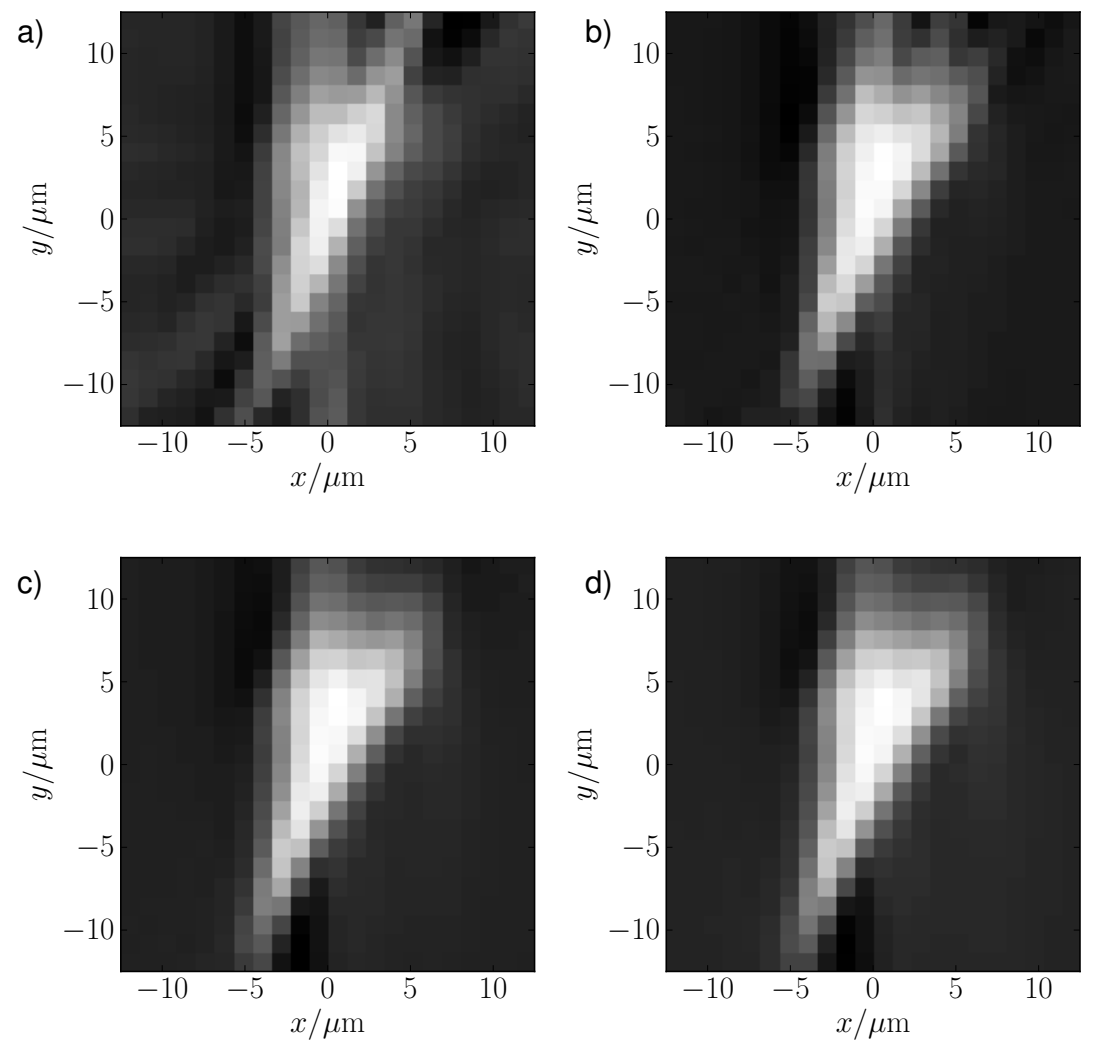

Figure 4.17: MCF simulated pore images of the hemi-equilateral triangular domain. Number of angular profiles acquired in $\boldsymbol{q}$-space was varied to $N_{\theta}=9$ (a), $N_{\theta}=18$ (b), $N_{\theta}=36$ (c), $N_{\theta}=54(\mathrm{~d})$.

increasing number of profiles the pore images become sharper and less artifacts due to the reconstruction are present. Figure 4.17 (a) would not be acceptable for experiments. However, all other images are acceptable and clearly show the triangular domain. Thus, the least acceptable number of profiles in this simulation were $N_{\theta}=18$.

\subsubsection{1d-MRPI results}

Following the preliminary MCF simulations discussed in the previous section, a 1d-MRPI experiment was performed on the triangular capillary sample TRI. The optimized set 
of parameters were $2 \tau=6 \mathrm{~ms}, N_{\mathrm{L}}=26$ and $\delta^{\prime}=5.5 \mathrm{~ms}$ leading to $T=168 \mathrm{~ms}$ and an effective narrow gradient duration of $2 \delta^{\prime}=11 \mathrm{~ms}$. Subsequently, a one-dimensional MRPI experiment was carried out along the direction indicated by the direction $\boldsymbol{q}$ in the insert of fig. 4.18. Figure 4.18 shows the real part (squares) and imaginary part (triangles) of the $\boldsymbol{q}$-space data as obtained from the center of the spin-echos for each gradient step. The solid and dashed line show the real and imaginary part as obtained by

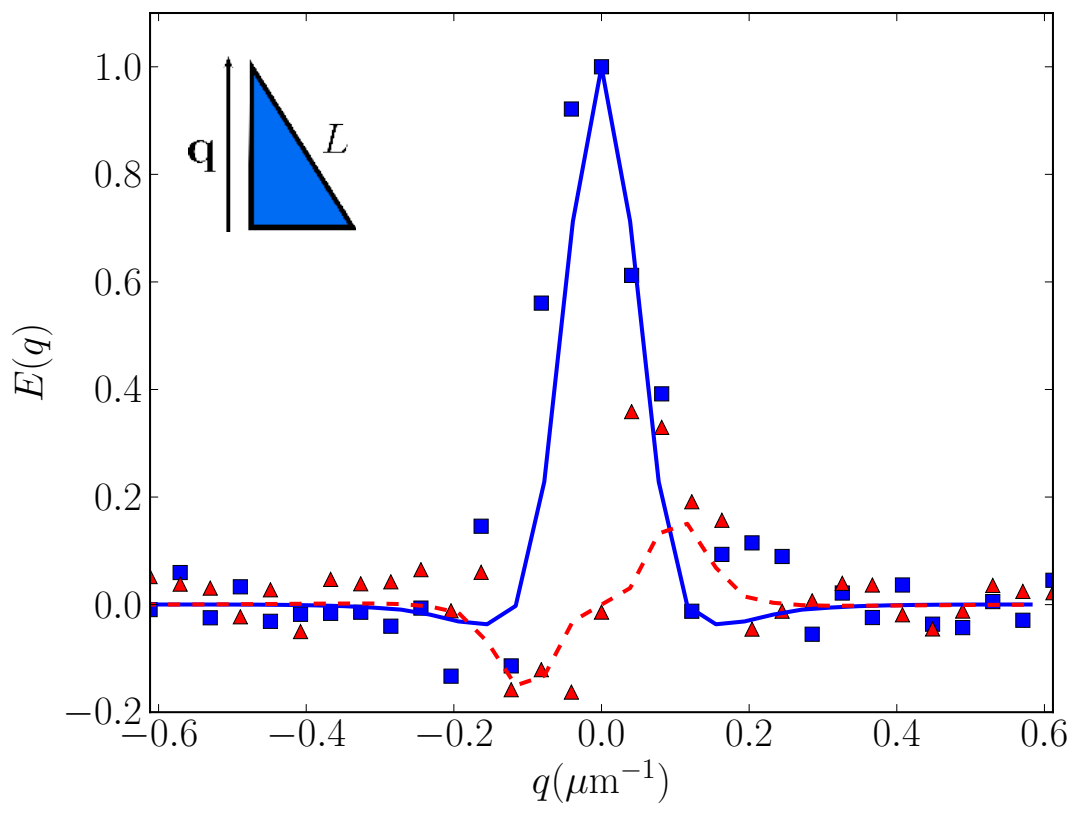

Figure 4.18: Comparison of experiment and simulation of the $q$-space profile as obtained when applying a one-dimensional MRPI experiment to the stack of hemi-equilateral triangular capillaries. The phase behavior of the real and imaginary parts of the signal are qualitatively in agreement. The deviations can be explained by irregularities in the capillary shape and the noise level inherent in the experimental data.

MCF simulation. Parameters of the MCF simulation were identical to the experimental parameters, however the gradient pulses were approximated to be rectangular, while their duration included one ramp time. Simulation and experiment agree qualitatively. Differences can be explained by residual irregularities in the capillary geometry due to the manufacturing process and the inherent noise in experimental data. It has to be considered that MRPI measures the average pore shape of all pores present in the sample. Therefore, any irregularities in the pore shape including rounded edges, slightly different sizes and any mismatch between the orientation of the triangular pores will be 
measured using MRPI. When considering the light microscope image shown in fig. 3.16, one may notice that such irregularities are present in the sample used in this work.

However, experiment and simulation show the correct phase behavior, which includes values different from $0^{\circ}$ and $180^{\circ}$. Thus, fig. 4.18 shows for the first time the measurement of the complex one dimensional form factor of a triangular domain on the micro-metre scale. These results show that the MRPI approach may be applied to arbitrary geometries of pores and thus resolves the "phase problem" for non-symmetric pores exhibiting form factors with arbitrary phases between the real and imaginary part.

Figure 4.19 shows the comparison between experiment (red dots) and simulation (black line) of the real space profiles as obtained by taking the magnitude of the Fourier transform of the $\boldsymbol{q}$-space data. In contrast to the profiles obtained for the cylindrical pores

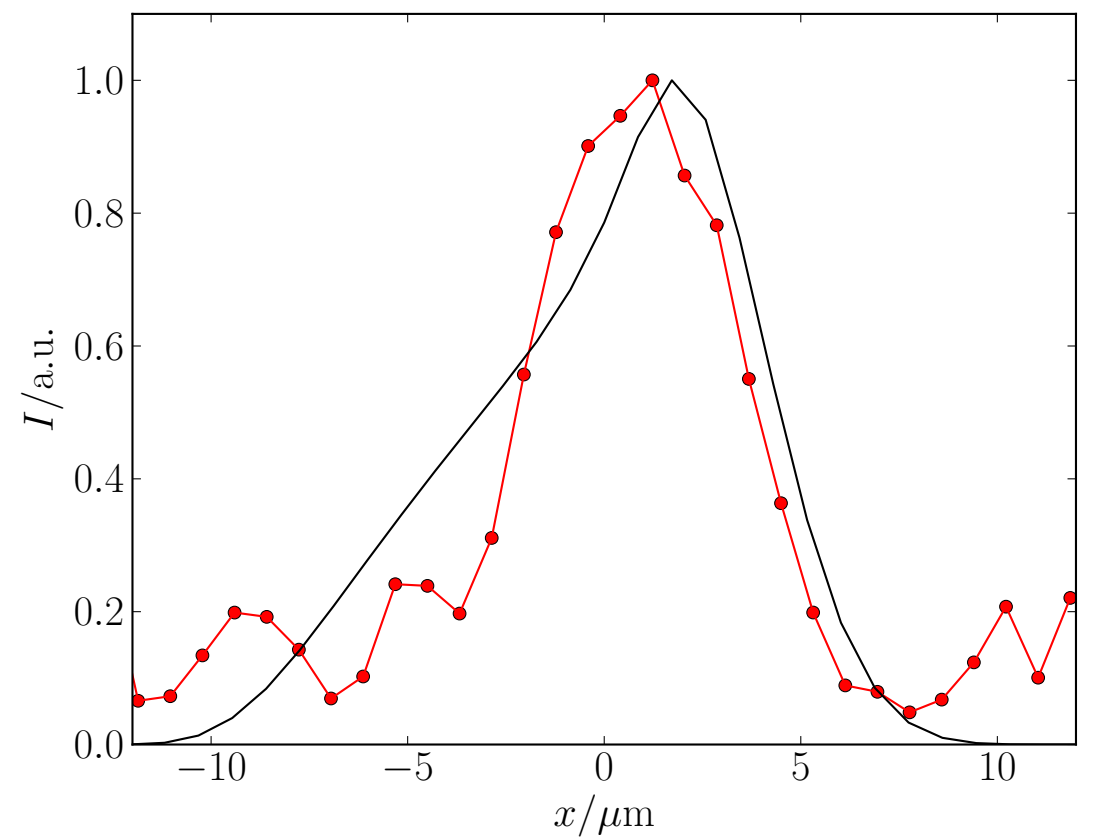

Figure 4.19: Comparison of the experimental one-dimensional profile of the hemi-equilateral triangular domain (red dots) and the MCF simulation (solid line). The profiles were obtained by taking the magnitude of the Fourier transform of the $\boldsymbol{q}$-space data. The magnitude of the data is shown here, since it is also used for for the $2 d$ inverse Radon transform as shown in sec. 4.3.3.

shown in fig. 4.9, the profiles shown here are non-symmetric. Thus, the reconstruction of the underlying non-symmetric pore shape becomes possible as will be shown in the next section. The nominal resolution of this experiment was $\Delta x=0.8 \mu \mathrm{m}$, which however will 
be reduced by blurring and edge enhancement as shown for the cylindrical capillaries in sec. 4.2.3. Furthermore, one may notice deviations between experiment and simulation, which have been discussed for the $\boldsymbol{q}$-space data in fig. 4.18 and are therefore present in the Fourier transform shown in fig. 4.19.

\subsubsection{2d-MRPI results}

The back-projection/Radon transform approach was chosen to perform 2d-MRPI experiments on the sample TRI as discussed in sec. 4.3. Due to the absorption of $\mathrm{H}_{2} \mathrm{O}$ molecules at the polycarbonate- $\mathrm{H}_{2} \mathrm{O}$ interface it was ensured that the pores remained filled throughout the set of experiments by MRI images as exemplary shown in fig. 3.18.

Experimental parameters as suggested by the MCF simulations discussed in sec. 4.3.1 were $N_{\mathrm{L}}=26, N_{\mathrm{N}}=2,2 \tau=6 \mathrm{~ms}, \delta^{\prime}=5.5 \mathrm{~ms}$ and the gradients were stepped from $G_{\min }=-1.45 \mathrm{Tm}^{-1}$ to $G_{\max }=1.45 \mathrm{Tm}^{-1}$ in 64 steps. The one-dimensional MRPI pulse sequence was applied in $N_{\theta}=18$ directions.

Each of the 18 acquired $\boldsymbol{q}$-space profiles was Fourier transformed and subsequently a back-projection algorithm was applied to yield the two-dimensional image. Figure 4.20 (a) shows the expected image for triangular capillaries where surface relaxation has been neglected. The image clearly shows the triangular shape of the underlying pore structure. Note that fig. 4.20 (a) is identical to fig. 4.17 (b) except for the rotation of the image, which was adjusted to reflect the orientation of the sample in the gradient system. Figure 4.20 (b) shows the experimental MRPI image. As a guide to the eye the expected triangular shape is indicated. The hemi-equilateral pore shape is recovered although with additional blurring due to the finite surface relaxation. Another major source of blurring may be heterogeneities in the pore shapes as can be seen from the light microscope image in fig. 3.16. Despite these deviations, fig. 4.20 (b) shows the elongated shape of the hemi-equilateral triangular pores. Moreover, both the simulated image and the experimental image show rounded edges in the images due to the blurring and edge enhancement.

We therefore proved for the first time the applicability of MRPI for non-point symmetric pore shapes. Furthermore, the presence of finite surface permeability and surface 

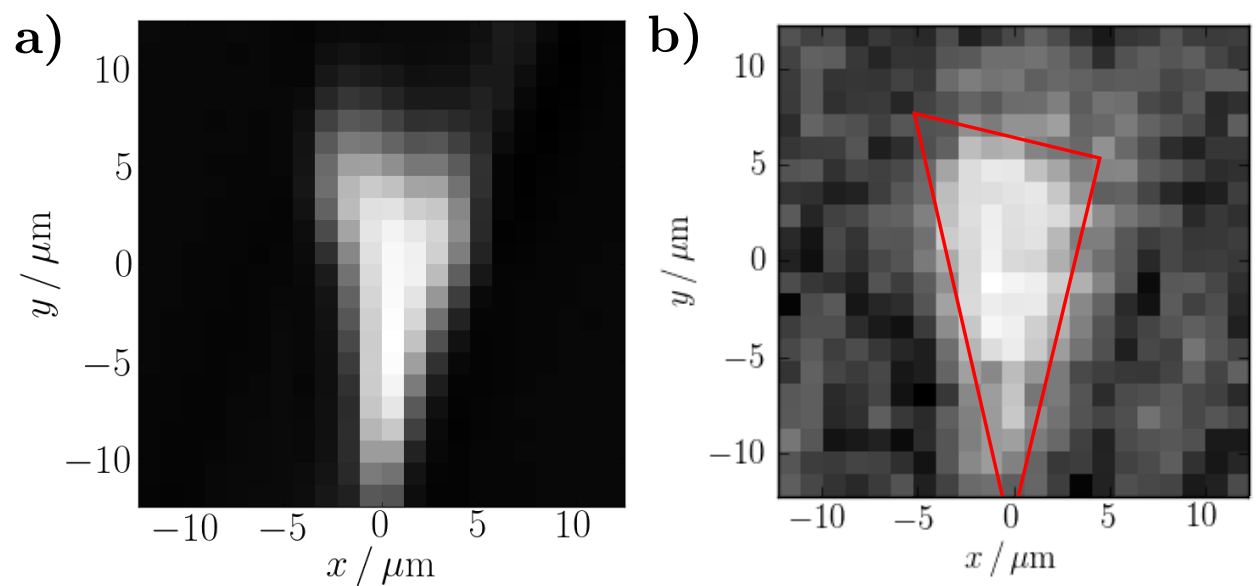

Figure 4.20: Image simulated with MCF technique (a) and experimental image obtained by Radon transform of the one-dimensional Fourier transformed $q$-space profiles (b).

relaxation did not pose any insurmountable barrier to the application of MRPI. In fact the presence of these effects in the polycarbonate sample may be regarded as a starting point for a systematic study of the effect of surface relaxivity and surface permeability and the required MRPI parameters. This may aid the method development for the further application of the method to natural porous media.

\subsection{MRPI mapping}

\subsubsection{MRPI 1d-mapping}

The MRPI mapping pulse sequence as shown in fig. 3.6 was applied to the capillary sample CYL2 with two compartments. A plot of the MRPI mapping data is shown in fig. 4.21 (Hertel et al. (2015a)). The horizontal dimension shows the one-dimensional spin density of the sample in $x$-direction, which was obtained by Fourier transform of the spin-echo signal in the time domain for each step in the MRPI gradients. The one-dimensional profile of the capillary stack with an extension of ca. $I D=8 \mathrm{~mm}$ is visible for each step in $\boldsymbol{q}$. The two compartments can be distinguished by a higher signal intensity for the side with the larger capillaries. It is worth noting that the higher signal intensity is solely caused by the larger volume of $\mathrm{H}_{2} \mathrm{O}$ present in the large capillaries rather than a higher number of capillaries per pixel. The vertical dimension shows the 


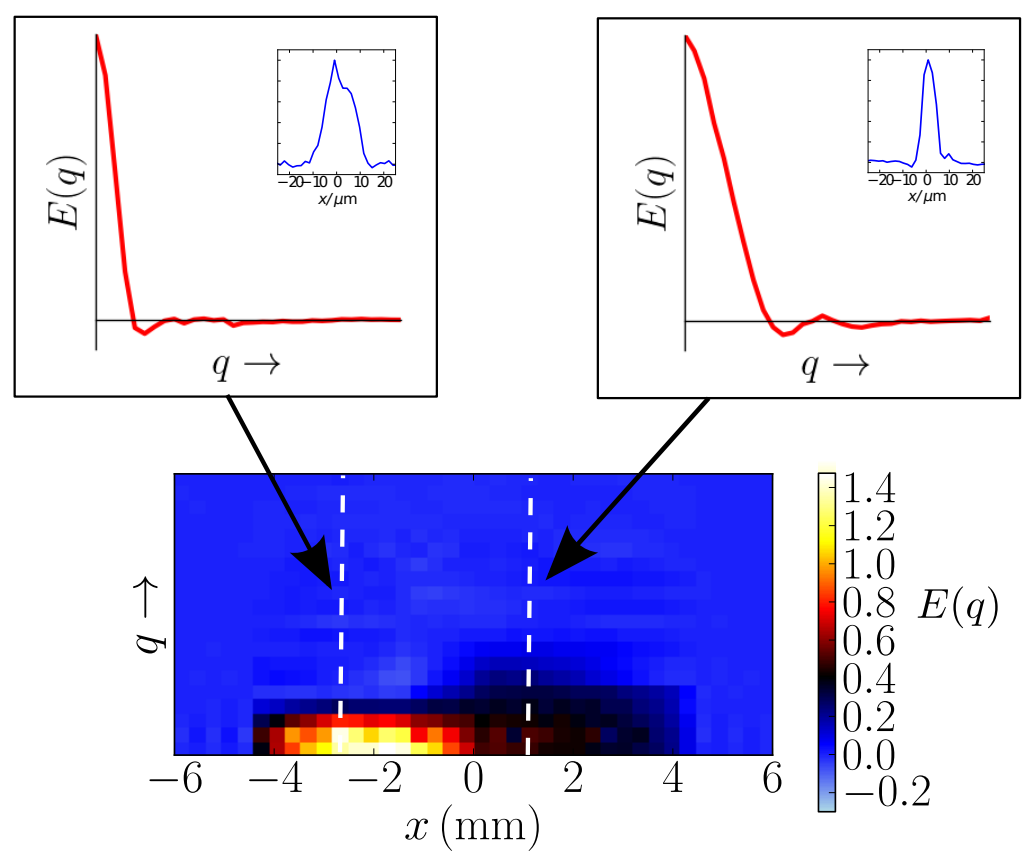

Figure 4.21: Plot of the results of the one-dimensional MRPI mapping experiment. Here, the Fourier transform of the spin echo in $x$-direction is plotted along the horizontal axis versus the MRPI $\boldsymbol{q}$ space encoding on the vertical axis (bottom). The one-dimensional profile of the capillary stack with an extension of ca. $I D=8 \mathrm{~mm}$ is visible for each step in $\boldsymbol{q}$ (bottom). Exemplary for two pixels the MRPI signal is extracted along the vertical dotted lines. One sees a shift of the zero crossings to higher $q$-values as is expected for the smaller pores (top). The inserts show the 1d-profiles of the pores obtained by Fourier transform of the $\boldsymbol{q}$ space profiles (top). Color scales are signal intensities in arbitrary units. Reprinted from Hertel et al. (2015a).

$\boldsymbol{q}$ space data. Profiles along the $\boldsymbol{q}$ direction are plotted for two exemplary pixels on the top of fig. 4.21. The form factors for the large and small capillaries are recovered as it is apparent by the shift of the zero-crossings to higher $q$-values for the side with the smaller pores. The inserts in fig. 4.21 (top) show the 1d-profiles of the pores obtained by Fourier transform of the $\boldsymbol{q}$ space profiles.

The experiments presented in this section were performed with the orientation of the sample in the gradient system, such that the compartment separation is perpendicular to the direction of the gradient $G_{\mathrm{R}}$. Therefore, the two compartments were clearly separated in the $1 \mathrm{~d}$ image. However, if the sample would have been rotated, one would measure a bi-modal distribution of pore sizes for each pixel where the two compartments overlap. In this case the analysis of the pore size distribution would be more complex. 
Moreover, the spatial distribution of pore sizes in the direction perpendicular to $G_{\mathrm{R}}$ is not obtainable using this approach. Therefore, the one-dimensional MRPI mapping experiment may provide a rapid measurement of the distribution of pore sizes, but the 2d-MRPI mapping experiment may provide further and more complete information.

\subsubsection{MRPI 2d-mapping}

The 2d-MRPI mapping pulse sequence as shown in fig. 3.7 was applied to the sample CYL2. Figure 4.22 (a) shows the two-dimensional MRI image of the cross-section of sample CYL2. This image was obtained by $2 \mathrm{~d}$-Fourier transform of the MRI encoded image for the first step in the MRPI gradient sequence $(\boldsymbol{q}=0)$. For higher values of $\boldsymbol{q}$ the capillary stack would still be visible, however with much reduced signal-to-noise due to signal attenuation. The two-dimensional matrix consisted of 512 points in the the time domain under influence of the read gradient $G_{\mathrm{R}}$ and 8 steps of the phase domain encoded by the phase gradient $G_{\mathrm{P}}$. The width of the circular profile in the MRI image agrees with the actual inner diameter of the sample tube of $I D=8 \mathrm{~mm}$. Figure 4.22 (c) shows a sketch of the transverse cross-section through the sample. Note that the sketch is not to scale and many more capillaries were contained in the sample tube. In the sketch, the direction $x$ indicates in which direction the read gradient $\boldsymbol{G}_{\mathrm{R}}$ was applied. The two compartments of capillary diameters can be distinguished by an increased signal intensity for the side with the larger capillaries. The MRPI signal was Fourier transformed for two exemplary pixels. The arrows indicate the location of the MRI pixels from which the pore images were extracted. Clearly, the dimensions of the large (fig. 4.22 (b)) and small capillaries (fig. 4.22 (d)) were recovered.

It is intriguing to see that the signal-to-noise of the profiles is on the order of 10 although only a few capillaries are present in each MRI voxel. This is a consequence of the increased signal-to-noise of MRPI as compared to diffusive-diffractive PGSE NMR, since $S_{0}(\boldsymbol{q})$ is measured instead of $\left|S_{0}(\boldsymbol{q})\right|^{2}$. This may render MRPI more robust and versatile for combinations with MRI in mapping experiments.

In principle one may perform 4 -dimensional $(2 \mathrm{~d} \times 2 \mathrm{~d})$ experiments where for each pixel of the MRI image a full 2-dimensional MRPI image is acquired. The resulting data set 


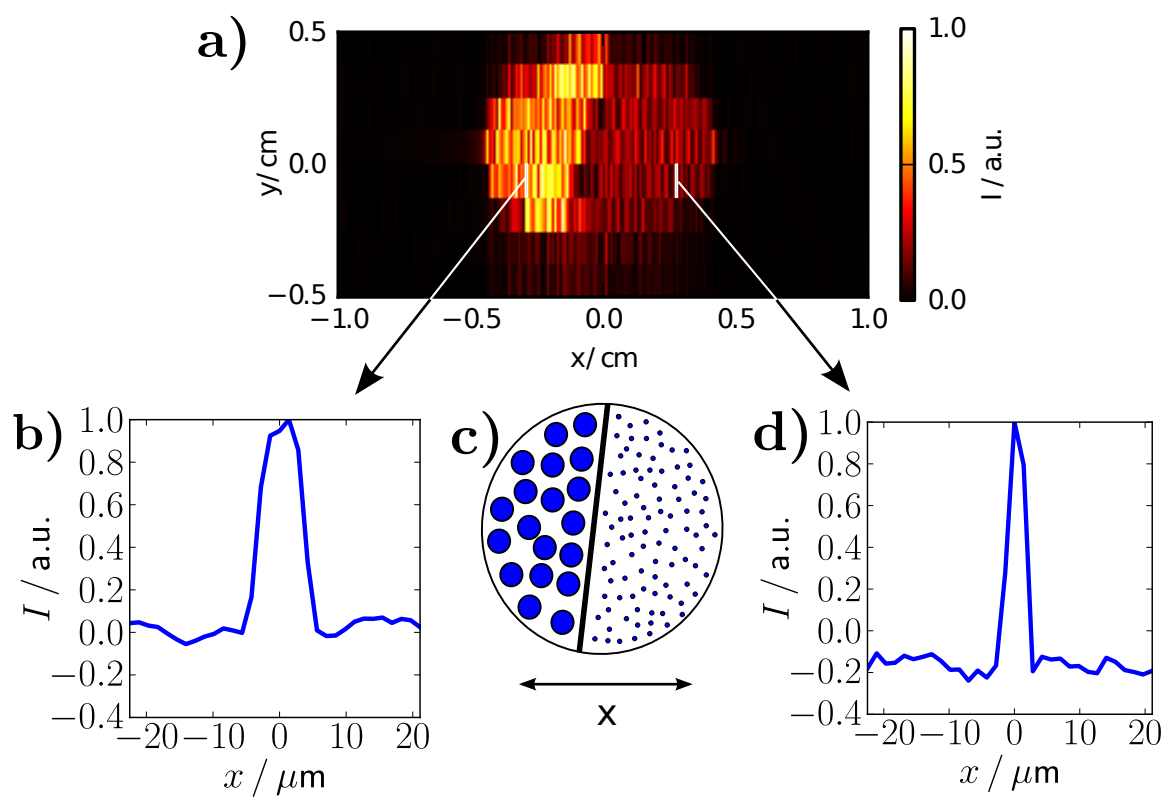

Figure 4.22: Mapping of average pore images on the MRI image. Shown are the two imaging dimensions $x$ and $y$. The MRPI encoding was applied in the third dimension (not shown). The Fourier transform of the MRPI $\boldsymbol{q}$-space profile is shown for two exemplary pixels (arrows). The profile widths yield the right pore diameters of $L_{1}=20 \mu \mathrm{m}$ (b) and $L_{2}=10 \mu \mathrm{m}(\mathrm{d})$. The sketch shows the cross-section through the whole sample and the two compartments filled with two sizes of cylindrical capillaries (c).

would be a 4 -dimensional matrix. The $2 \mathrm{~d}$-Fourier transform of the MRI $k$-space data at zero MRPI gradient $(\boldsymbol{q}=0)$ yields the MRI image. Another 2d-Fourier transform of the $q$-space data yields the average pore image for each voxel in the MRI image. However, the cylindrical symmetry of the underlying pore structure would not yield any additional information for the sample used in this study and a $3 \mathrm{~d}$ approach is sufficient in this case. Thus, one is able to map the average pore shapes as well as sizes present within a given voxel onto MRI images, which allows to combine the resolution of MRPI with the spatial information of MRI. 



\section{Conclusions}

In this thesis, it has been experimentally shown that the MRPI approach lifts fundamental limitations of diffusive-diffraction PGSE NMR. By creating a hybrid between MRI and PGSE NMR we could show that it is possible to obtain full structural information about model porous materials. Since MRPI measures the phase of the NMR signal, it allows the direct Fourier inversion of the experimental data to obtain average pore images. In this thesis one key innovation was the adaptation of a CPMG based design for the MRPI pulse sequence. This allowed us to extend the long gradient of the MRPI pulse sequence to fulfill the condition that the molecules on average traverse the pore space multiple times during its application.

The first proof of the applicability of MRPI was achieved with a stack of cylindrical capillaries with a common radius of $L=10 \mu \mathrm{m}$. The acquired $\boldsymbol{q}$-space profiles contained the negative lobes of the cylindrical form factor, while diffusive-diffraction PGSE NMR only measures positive values. Moreover, the signal-to-noise with MRPI is improved by up to an order of magnitude as compared to diffusive-diffraction PGSE NMR, since $\overline{S_{0}(\boldsymbol{q})}$ is measured instead of $\overline{\left|S_{0}(\boldsymbol{q})\right|^{2}}$.

Investigations about the required convergence times revealed that MRPI may need considerably more time for the convergence to the form factor, than diffusive-diffraction PGSE NMR needs to converge to the absolute square of the form factor. While for diffusive-diffraction PGSE NMR tens of milliseconds may be sufficient, MRPI may require a minimum of one hundred millisecond to extract useful information about the pore space. However, if the condition for convergence is met for MRPI one is rewarded by the greater information content of the average form factor.

Further considerations on the limits and applicability of the method are discussed in 
Laun et al. (2012). It depends on several experimental factors such as pore diameter, the mobility of the probe molecules or available gradient strength. Generally one needs to satisfy that molecules diffuse several times across the pore while maintaining a small diffusive displacement during the narrow gradient. Despite these challenges, further development of the method and the careful choice of the pore space fluid (from gases to viscous hydrocarbons) may allow to apply MRPI broadly.

We introduced the possibility to traverse $\boldsymbol{q}$-space on a Cartesian grid and thus to extend the method to 2d-MRPI. Once the $\boldsymbol{q}$-space data is acquired, we could show that a simple 2d-Fourier transform yields the average pore image of the sample under study. For the cylindrical capillaries we acquired $2 \mathrm{~d}$ pore images with an unprecedented resolution of $1.3 \mu \mathrm{m}$. The $2 \mathrm{~d}$ approach allows to image the pore shape directly without any prior assumptions. It is therefore generally superior to the $1 \mathrm{~d}$ approach, which may require the comparison of the profiles with theory to allow conclusions about the shape. Furthermore, we could show that the MCF simulation technique can be adapted to the CPMG based gradient scheme of the MRPI pulse sequence. A comparison with a cross section of the experimental $2 \mathrm{~d}$ pore image shows that the effects of blurring and edge enhancement are correctly captured using this simulation technique.

Another challenge for establishing MRPI was the application to non-symmetric pore shapes. Therefore, a fabrication method had to be found to produce capillaries of triangular shape on the micrometer scale. We met this challenge in cooperation with the Photon Factory of Auckland University. A stack of hemi-equilateral triangular capillaries with a characteristic width of $L=20 \mu \mathrm{m}$ was created by laser micro-machining. Utilizing these samples we could show that MRPI enables the measurement of the signal with arbitrary phase which is different from 0 and $180^{\circ}$. Since these samples exhibit a finite absorption probability of the water molecules on the pore boundaries we applied the back projection approach to acquire $2 \mathrm{~d}$ average pore images. This approach allows to check the sample condition in between the acquisition of $1 \mathrm{~d} \boldsymbol{q}$-space profiles and therefore it could be ensured that the capillaries stayed filled during the experiments. The $2 \mathrm{~d}$ pore images show the hemi-equilateral triangular pore shape and provide experimental proof that MRPI can be applied to pores with arbitrary symmetry. 
Natural porous materials may contain distributions of pore sizes and shapes. In this thesis, we introduced the MRPI mapping experiment, which allows to measure the MRPI signal inside an individual voxel of an MRI image. This way the spatial information of the MRI image which extends over the whole sample can be combined with the local microscopic information obtained with the MRPI approach. The proof of concept was shown on a sample containing two compartments filled with cylindrical capillaries of different sizes. By combining a 2d-MRI pulse sequence and the MRPI pulse sequence we could measure the profile of the cylindrical pores in individual voxels, which correctly returned the bi-modal distribution of pore sizes. While methods for the mapping of local pore sizes have been introduced (Komlosh et al. (2011), Shemesh et al. (2013)), MRPI mapping provides the local averaged pore shape as the more fundamental characterization of the porous system.

\subsection{Future work}

One interesting application of MRPI would be the imaging of biological porous media such as yeast cells, nerve bundles or cancer cells. Some preliminary considerations suggest that such experiments may pose additional experimental challenges, although if successful they would be very rewarding. For example, cell walls may be partially permeable to water molecules and thus the condition of diffusing several times across the pore space may not be met. However, this challenge may be overcome by utilizing the nuclear magnetization of other molecules such as $N$-acetylaspartic acid (NAA) (Laun et al. (2012)). NAA can only be found in the soma of neurons, but not outside of the neurons and may therefore be an ideal candidate for MRPI studies. If successful these studies could open new avenues to study biological processes and to gain a deeper understanding of various diseases. Other promising applications could be the study of the epithelial cell structure of plants and the imaging of rock fissures in oil bearing reservoirs.

Moreover, it was shown that MRPI integrates seamlessly with proven concepts of MRI. Therefore, one may expect to find advantages and established approaches of MRI to 
work for MRPI as well. This may include efficient ways to sample $\boldsymbol{q}$-space by employing advanced schemes such as sparse or spiral sampling as known from conventional MRI. This may reduce the required time to acquire average pore images, which would be necessary for applications like medical screening.

Another possibility is to utilize additional information which can be encoded in the NMR signal. For example, one may design pulse sequences with segments where the magnetization is stored in $z$-direction to create contrast relying on longitudinal relaxation times $T_{1}$. A second example, would be the utilization of chemical shift information for highlighting parts of the pore images with different magnetic susceptibilities. Furthermore, although the method is most promising for closed pore systems it maybe applicable for connected pore spaces in selected cases (Callaghan (2011). 


\section{Bibliography}

Abragam, A. (1961). Principles of Nuclear Magnetism. Clarendon Press, Oxford.

Als-Nielsen, J. and McMorrow, D. (2011). Elements of Modern X-ray Physics. Wiley, Chichester.

Álvarez, G. A., Shemesh, N., and Frydman, L. (2013). Coherent dynamical recoupling of diffusion-driven decoherence in magnetic resonance. Phys. Rev. Lett., 111:080404.

Appel, M. and Fleischer, G. (1993). Investigation of the chain-length dependence of selfdiffusion of poly(dimethylsiloxane) and poly(ethylene oxide) in the melt with pulsedfield gradient NMR. Macromolecules, 26(20):5520-5525.

Bain, A. D. (1984). Coherence levels and coherence pathways in NMR. A simple way to design phase cycling procedures. J. Magn. Reson. 1969, 56(3):418-427.

Baltisberger, J. H., Walder, B. J., Keeler, E. G., Kaseman, D. C., Sanders, K. J., and Grandinetti, P. J. (2012). Phase incremented echo train acquisition in NMR spectroscopy. J. Chem. Phys., 136(21):211104.

Bar-Shir, A., Avram, L., Özarslan, E., Basser, P. J., and Cohen, Y. (2008). The effect of the diffusion time and pulse gradient duration ratio on the diffraction pattern and the structural information estimated from q-space diffusion MR: Experiments and simulations. J. Magn. Reson., 194(2):230-236.

Barrall, G. A., Frydman, L., and Chingas, G. C. (1992). NMR diffraction and spatial statistics of stationary systems. Science, 255(5045):714-717. 
Bax, A., De Jong, P., Mehlkopf, A., and Smidt, J. (1980). Separation of the different orders of NMR multiple-quantum transitions by the use of pulsed field gradients. Chem. Phys. Lett., 69(3):567-570.

Benjamini, D. and Nevo, U. (2013). Estimation of pore size distribution using concentric double pulsed-field gradient NMR. J. Magn. Reson., 230(0):198-204.

Berne, B. J. and Pecora, R. (2000). Dynamic Light Scattering. Dover Publications Inc.

Bernstein, M. A., King, K. F., and Zhou, X. J. (2004). Handbook of MRI Pulse Sequences. Elsevier Academic.

Bernstein, M. A., Zhou, X. J., Polzin, J. A., King, K. F., Ganin, A., Pelc, N. J., and Glover, G. H. (1998). Concomitant gradient terms in phase contrast MR: Analysis and correction. Magn. Reson. Med., 39(2):300-308.

Bloch, F. (1946). Nuclear induction. Phys. Rev., 70(7-8):460-474.

Bloembergen, N., Purcell, E. M., and Pound, R. V. (1948). Relaxation effects in nuclear magnetic resonance absorption. Phys. Rev., 73(7):679-712.

Bodenhausen, G., Kogler, H., and Ernst, R. (1984). Selection of coherence-transfer pathways in NMR pulse experiments. J. Magn. Reson. 1969, 58(3):370-388.

Callaghan, P. (1995). Pulsed-gradient spin-echo NMR for planar, cylindrical, and spherical pores under conditions of wall relaxation. J. Magn. Reson. A, 113(1):53-59.

Callaghan, P., Coy, A., Forde, L., and Rofe, C. (1993). Diffusive relaxation and edge enhancement in NMR microscopy. J. Magn. Reson. A, 101(3):347-350.

Callaghan, P., Coy, A., Macgowan, D., Packer, K. J., and Zelaya, F. O. (1991). Diffraction-like effects in NMR diffusion studies of fluids in porous solids. Nature, 351(6326):467.

Callaghan, P. T. (1990). PGSE-MASSEY, a sequence for overcoming phase instability in very-high-gradient spin-echo NMR. J. Magn. Reson., 88:493-500. 
Callaghan, P. T. (1991). Principles of Nuclear Magnetic Resonance Microscopy. Clarendon Press, Oxford.

Callaghan, P. T. (1997). A simple matrix formalism for spin echo analysis of restricted diffusion under generalized gradient waveforms. J. Magn. Reson., 129(1):74-84.

Callaghan, P. T. (2011). Translational Dynamics \& Magnetic Resonance. Oxford University Press, Oxford.

Callaghan, P. T. and Stepisnik, J. (1995). Frequency-domain analysis of spin motion using modulated-gradient NMR. J. Magn. Reson. A, 117(1):118-122.

Carr, H. Y. and Purcell, E. M. (1954). Effects of diffusion on free precession in nuclear magnetic resonance experiments. Phys. Rev., 94(3):630-638.

Cory, D. G. and Garroway, A. N. (1990). Measurement of translational displacement probabilities by NMR: An indicator of compartmentation. Magn. Reson. Med., 14(3):435-444.

Cotts, R. M. (1991). Diffusion and diffraction. Nature, 351(6326):443-444.

Crank, J. (1975). The mathematics of diffusion. University Press, Oxford.

De Swiet, T. (1995). Diffusive edge enhancement in imaging. J. Magn. Reson. B, 109(1):12-18.

Douglass, D. C. and McCall, D. W. (1958). Diffusion in paraffin hydrocarbons. J. Phys Chem., 62(9):1102-1107.

Dullien, F. A. L. (1992). Porous Media: Fluid Transport and Pore Structure. Academic Press, Inc., 2 edition.

Fick, A. (1855). Ueber diffusion. Annalen der Physik, 170(1):59-86.

Galvosas, P., Stallmach, F., Seiffert, G., Kärger, J., Kaess, U., and Majer, G. (2001). Generation and application of ultra-high-intensity magnetic field gradient pulses for NMR spectroscopy. J. Magn. Reson., 151(2):260-268. 
Grebenkov, D. and Nguyen, B.-T. (2013). Geometrical structure of Laplacian eigenfunctions. SIAM Rev., 55:601-667.

Grebenkov, D. S. (2006). Focus on Probability Theory. Nova Science Publishers, Inc.

Grebenkov, D. S. (2007). NMR survey of reflected Brownian motion. Rev. Mod. Phys., 79:1077-1137.

Grebenkov, D. S. (2008). Laplacian eigenfunctions in NMR. I. a numerical tool. Concepts Magn. Reson. A, 32A(4):277-301.

Hahn, E. L. (1950). Spin echos. Phys. Rev., 80(4):580-594.

Hertel, S. and Galvosas, P. (2015). Tailoring phase cycles using phase incremented echo train acquisition. J. Magn. Reson., pages -. in preparation.

Hertel, S., Hunter, M., and Galvosas, P. (2013). Magnetic resonance pore imaging, a tool for porous media research. Phys. Rev. E, 87:030802.

Hertel, S., Hunter, M., and Galvosas, P. (2015a). Magnetic resonance pore imaging: Overcoming the resolution limit of MRI for closed pore systems. Microporous and Mesoporous Materials, 205:44-47.

Hertel, S., Wang, X., Coard, P., Cather, S., Hunter, M., and Galvosas, P. (2015b). Magnetic resonance pore imaging of microscopic non-symmetric pore shapes. Phys. Rev. E. minor revisions.

Hidalgo-Tobon, S. (2010). Theory of gradient coil design methods for magnetic resonance imaging. Concepts Magn. Reson. A, 36A(4):223-242.

Hocheng, H. and Wang, K.-Y. (2008). Microgroove pattern machined by excimer laser dragging. Int. J. Manuf. Techol. Manag., 13(2/3/4):241-253.

Holz, M., Heil, S. R., and Sacco, A. (2000). Temperature dependent self-diffusion coefficients of water and six selected molecular liquids for calibration in accurate ${ }^{1} \mathrm{H}$ NMR PFG-measurements. Phys. Chem. Chem. Phys., 2:4740. 
Holz, M. and Weingärtner, H. (1991). Calibration in accurate spin-echo self-diffusion measurements using ${ }^{1} \mathrm{H}$ and less-common nuclei. J. Magn. Reson., 92(1):115-125.

Hrovat, M. I. and Wade, C. G. (1980). Absolute measurements of diffusion coefficients by pulsed nuclear magnetic resonance. J. Chem. Phys., 73(5):2509-2510.

Hürlimann, M. D. (1998). Effective gradients in porous media due to susceptibility differences. J. Magn. Reson., 131(2):232-240.

Hürlimann, M. D. (2001). Diffusion and relaxation effects in general stray field NMR experiments. J. Magn. Reson., 148(2):367-378.

Jenista, E. R., Stokes, A. M., Branca, R. T., and Warren, W. S. (2009). Optimized, unequal pulse spacing in multiple echo sequences improves refocusing in magnetic resonance. J. Chem. Phys., 131(20):204510.

Jobic, H. (2000). Diffusion studies using quasi-elastic neutron scattering. In Kanellopoulos, N., editor, Recent advances in gas separation by microporous ceramic membranes, volume 6, pages 109-137. Elsevier.

Kalies, G. (2009). Charakterisierung von porösen festkörpern mittels adsorption. Lecture.

Kärger, J. and Heink, W. (1983). The propagator representation of molecular transport in microporous crystallites. J. Magn. Reson., 51:1-7.

Kärger, J., Pfeifer, H., and Heink, W. (1988). Principles and application of self-diffusion measurements by nuclear magnetic resonance. Adv. Magn. Reson., 12:1-89.

Karlicek, R. F. and Lowe, I. J. (1980). A modified pulsed gradient technique for measuring diffusion in the presence of large background gradients. J. Magn. Reson., 37:75-91.

Kiselev, V. G. and Novikov, D. S. (2013). Comment on "magnetic resonance imaging by synergistic diffusion-diffraction patterns". Phys. Rev. Lett., 110:109801. 
Komlosh, M. E., Özarslan, E., Lizak, M. J., Horkay, F., Schram, V., Shemesh, N., Cohen, Y., and Basser, P. J. (2011). Pore diameter mapping using double pulsed-field gradient MRI and its validation using a novel glass capillary array phantom. J. Magn. Reson., 208(1):128-135.

Kuder, T. A., Bachert, P., Windschuh, J., and Laun, F. B. (2013). Diffusion pore imaging by hyperpolarized xenon-129 nuclear magnetic resonance. Phys. Rev. Lett., 111:028101.

Kuder, T. A. and Laun, F. B. (2012). NMR-based diffusion pore imaging by double wave vector measurements. Magn. Reson. Med., pages 836-841.

Laun, F. B., Kuder, T. A., Semmler, W., and Stieltjes, B. (2011). Determination of the defining boundary in nuclear magnetic resonance diffusion experiments. Phys. Rev. Lett., 107:048102.

Laun, F. B., Kuder, T. A., Wetscherek, A., Stieltjes, B., and Semmler, W. (2012). NMR-based diffusion pore imaging. Phys. Rev. E, 86:021906.

Mansfield, P. (1984). Spatial mapping of the chemical shift in NMR. Magn. Reson. Med., 1(3):370-386.

Mansfield, P. and Grannell, P. K. (1975). "diffraction" and microscopy in solids and liquids by NMR. Phys. Rev. B, 12(9):3618-3634.

Mansfield, P. and Morris, P. (1982). NMR Imaging in Biomedicine, volume 12, Supplement 2 of Advances in Magnetic Resonance. Academic Press, New York.

Mansfield, P. and Pykett, I. L. (1978). Biological and medical imaging by NMR. J. Magn. Reson., 29:355.

Meiboom, S. and Gill, D. (1958). Modified spin-echo method for measuring nuclear relaxation times. Rev. Sci. Instrum., 29(8):688-691.

Mills, R. (1973). Self-diffusion in normal and heavy water in the range 1-45.deg. J. Phys Chem., 77(5):685-688. 
Mitra, P. P. and Halperin, B. I. (1995). Effects of finite gradient-pulse widths in pulsedfield-gradient diffusion measurements. J. Magn. Reson. A, 113(1):94-101.

Neuman, C. H. (1974). Spin echo of spins diffusing in a bounded medium. J. Chem. Phys., 60(11):4508-4511.

Norris, D. G. and Hutchison, J. M. (1990). Concomitant magnetic field gradients and their effects on imaging at low magnetic field strengths. Magn. Reson. Imag., 8(1):3337.

Ogonczyk, D., Wegrzyn, J., Jankowski, P., Dabrowski, B., and Garstecki, P. (2010). Bonding of microfluidic devices fabricated in polycarbonate. Lab on a Chip, 10:13241327.

Price, W. S. (1998). Pulsed-field gradient nuclear magnetic resonance as a tool for studying translational diffusion: Part II. experimental aspects. Concepts Magn. Reson., 10:197-237.

Price, W. S. and Kuchel, P. W. (1991). Effect of nonrectangular field gradient pulses in the Stejskal and Tanner (diffusion) pulse sequence. J. Magn. Reson., 94(1):133-139.

Pütz, B., Barsky, D., and Schulten, K. (1992). Edge enhancement by diffusion in microscopic magnetic resonance imaging. J. Magn. Reson. 1969, 97(1):27-53.

Pütz, B., D., B., and Schulten, K. (1991). Edge enhancement by diffusion: microscopic magnetic resonance imaging of an ultrathin glass capillary. Chem. Phys. Lett., 183(5):391-396.

Rabi, I. I., Ramsey, N. F., and Schwinger, J. (1954). Use of rotating coordinates in magnetic resonance problems. Rev. Mod. Phys., 26:167-171.

Rabi, I. I., Zacharias, J. R., Millman, S., and Kusch, P. (1938). A new method of measuring nuclear magnetic moments. Phys. Rev., 53(4):318.

Robertson, B. (1966). Spin-echo decay of spins diffusing in a bounded region. Phys. Rev., 151:273-277. 
Rouquerol, J., Avnir, D., Fairbridge, C. W., Everett, D. H., Haynes, J. H., Pernicone, N., D.F., R. J., W., S. K. S., and Unger, K. K. (1994). Recommendations for the characterization of porous solids. Pure and Applied Chemistry, 66:1738-1758.

Ruthven, D. M. (1984). Principles of Adsorption and Adsorption Processes. John Wiley \& Sons.

Shannon, C. E. (1949). Communication in the presence of noise. Proc. Institute of Radio Engineers, 37:10-12.

Shemesh, N., Álvarez, G. A., and Frydman, L. (2013). Measuring small compartment dimensions by probing diffusion dynamics via non-uniform oscillating-gradient spinecho (NOGSE) NMR. J. Magn. Reson., 237(0):49-62.

Shemesh, N., Özarslan, E., Bar-Shir, A., Basser, P. J., and Cohen, Y. (2009). Observation of restricted diffusion in the presence of a free diffusion compartment: Singleand double-pfg experiments. J. Magn. Reson., 200(2):214-225.

Shemesh, N., Westin, C.-F., and Cohen, Y. (2012). Magnetic resonance imaging by synergistic diffusion-diffraction patterns. Phys. Rev. Lett., 108:058103.

Slichter, C. P. (1996). Principles of Magnetic Resonance. Springer, 3 edition.

Song, Y. Q. (2002). Categories of coherence pathways for the cpmg sequence. J. Magn. Reson., 157(1):82-91.

Stallmach, F. and Galvosas, P. (2007). Spin echo NMR diffusion studies. In Annu. Rep. NMR Spectrosc., volume 61, pages 51-131. Elsevier, Amsterdam, Boston, Heidelberg.

Stejskal, E. O. (1965). Use of spin echoes in a pulsed magnetic-field gradient to study anisotropic, restricted diffusion and flow. J. Chem. Phys., 43(10):3597-3603.

Stejskal, E. O. and Tanner, J. E. (1965). Spin diffusion measurements: Spin echoes in the presence of a time-dependent field gradient. J. Chem. Phys., 42(1):288-292.

Svergun, D., Fĕgin, L., and Taylor, G. (1987). Structure Analysis by Small-Angle X-Ray and Neutron Scattering. Plenum Press, New York. 
Tofts, P., Lloyd, D., Clark, C., Barker, G., Parker, G., McConville, P., Baldock, C., and Pope, J. (2000). Test liquids for quantitative MRI measurements of self-diffusion coefficient in vivo. Magn. Reson. Med., 43(3):368-374.

Torrey, H. C. (1956). Bloch equations with diffusion terms. Phys. Rev., 104(3):563-565.

Toumelin, E., Torres-Verdin, C., Sun, B., and Dunn, K.-J. (2007). Random-walk technique for simulating NMR measurements and 2d NMR maps of porous media with relaxing and permeable boundaries. J. Magn. Reson., 188(1):83-96.

Volegov, P., Mosher, J., Espy, M., and Kraus Jr., R. (2005). On concomitant gradients in low-field MRI. J. Magn. Reson., 175(1):103-113.

Woessner, D. E. (1960). Self-diffusion measurements in liquids by the spin-echo technique. Rev. Sci. Instrum., 31(10):1146-1146.

Woessner, D. E. (1963). NMR spin-echo self-diffusion measurements on fluids undergoing restricted diffusion. J. Phys. Chem., 67(6):1365-1366. 